Supporting Information

\title{
Reductive C-C Coupling by Desulfurizing Gold-Catalyzed Photoreactions
}

Lumin Zhang, ${ }^{\dagger}$ Xiaojia Si, ${ }^{\dagger}$ Yangyang Yang, ${ }^{\dagger}$ Sina Witzel, ${ }^{\dagger}$ Kohei Sekine, ${ }^{\dagger}$ Matthias Rudolph, ${ }^{\dagger}$ Frank Rominger, ${ }^{\dagger}$ A. Stephen K. Hashmi ${ }^{\dagger}, \star, * *$

$†$ Organisch-Chemisches Institut, Heidelberg University, Im Neuenheimer Feld 270, 69120 Heidelberg, Germany

+ Chemistry Department, Faculty of Science, King Abdulaziz University (KAU), Jeddah 21589, Saudi Arabia

*email address: hashmi@hashmi.de 


\section{Table of Contents}

I. General information...................................................1

II. General procedure for synthesis the substrates.........................3

III. Optimization of the reaction conditions...................................7

IV. General procedure for the C-C coupling by desulfurizing gold-catalyzed photoreaction..............................................................10

V. Mechanistic studies..................................................28

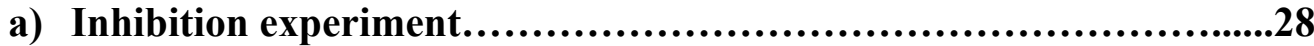

b) Radical clock reaction...............................................28

c) Deuterium experiment..............................................29

d) Control experiments...................................................30

e) Time profile........................................................... 33

f) $\mathrm{UV}$-vis spectra.....................................................33

g) Quantum yield measurements.............................................................35

h) Oxidation $\mathrm{Ph}_{3} \mathrm{P}$ with $\mathrm{O}_{2}$ in the presence of gold-catalyst................36

i) Oxidation $\mathrm{Ph}_{3} \mathrm{P}$ with 4-iodoanisole in the presence of gold-catalyst ..37

j) Stoichiometric experiment and NMR spectra identifications...........37

k) Visualized photoreaction............................................39

1) Detection newly formed gold complex by NMR spectra ................41

m) Filter experiments...................................................44

n) Using phosphate buffer solution as only solvent......................44

o) Gram scale synthesis..............................................45

p) Attempt with an unactivated aliphatic alkene as coupling partner.....45

q) Failure attempts for react methyl bromoacetate with styrene.........46

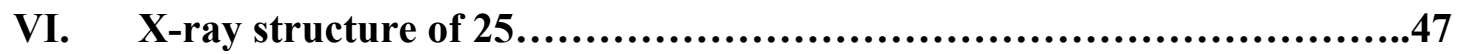

VII. References.............................................................48

VIII. NMR spectra.............................................................5 


\section{General Information}

All the reactions were performed under nitrogen atmosphere. Chemicals (Aldrich, Fluka, TCI, Alfa, Abcr, and Merck) were used without further purification. DCM, $\mathrm{MeOH}$, Toluene and $\mathrm{MeCN}$ were obtained from dried machine. The other solvents used directly from commercial reagents. NMR spectra were recorded on Bruker, Avance 300 (300 MHz), Avance $400(400 \mathrm{MHz})$ and Avance $500(500 \mathrm{MHz})$ spectrometers. Chemical shifts were referenced to residual solvent protons or TMS as internal standard and reported in ppm. The following abbreviations were used for ${ }^{1} \mathrm{H}$ NMR spectra to indicate the signal multiplicity: s (singlet); brs (broad singlet), d (doublet), t (triplet), $\mathrm{q}$ (quartet), quint (quintet), sext (sextet), sept (septet) and m (multiplet) as well as combinations of them. All ${ }^{13} \mathrm{C}$ NMR spectra were measured with ${ }^{1} \mathrm{H}$-decoupling. The multiplicities mentioned in these spectra [s (singlet, quaternary carbon), $\mathrm{d}$ (doublet, $\mathrm{CH}-$ group), $\mathrm{t}$ (triplet, $\mathrm{CH}_{2}$-group), q (quartet, $\mathrm{CH}_{3}$-group)] were determined by DEPT-135 or HSQC spectra. For fluorine containing compounds, the second multiplicity refers to the ${ }^{19}$ F-coupling. IR spectra were recorded on a Bruker Vector 22, and the absorption maxima were given in wavelength in $\mathrm{cm}^{-1}$ units. Thin-layer chromatography (TLC) was performed on precoated polyester sheets (POLYGRAM SIL /GUV254), and components were visualized by observation under UV light or by treating the plates with phosphomolybdic acid hydrate followed by heating. 


\section{Reaction equipment}

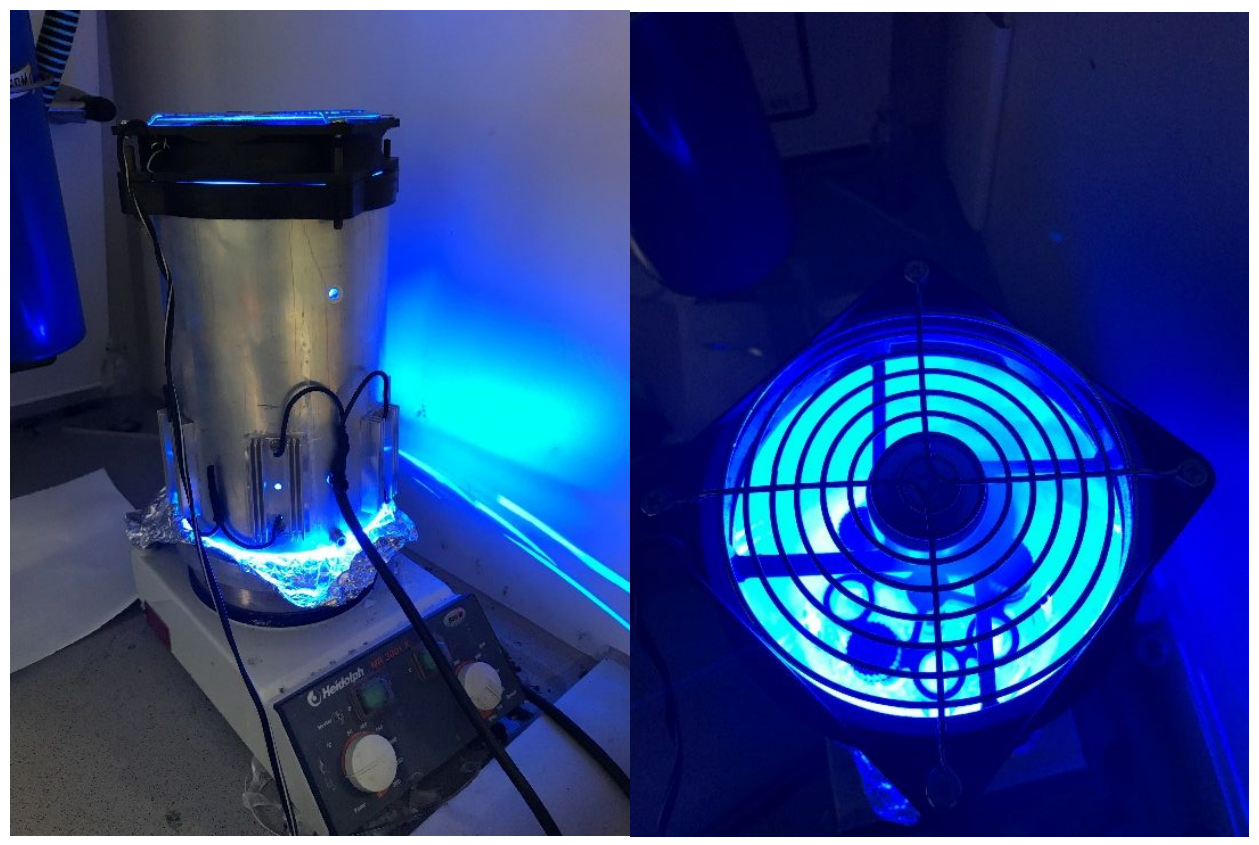

The photoreactor is equipped with a fan at the top to keep the reactor at ambient temperature during the reaction processes, and use $12 \mathrm{~W}$ LED strip $(\lambda \max =465 \mathrm{~nm})$. 


\section{General information for synthesis of the substrates}

Relevant known Alkene substrates $1 \mathbf{f}^{1}, \mathbf{1 m}^{2}, \mathbf{1} \mathbf{n}^{3}, \mathbf{1 0}^{4}, \mathbf{1} \mathbf{v}^{4}, \mathbf{1 w}^{4}, \mathbf{1} \mathbf{x}^{4}, \mathbf{1 y}^{4}, \mathbf{1} \mathbf{z}^{5}, \mathbf{8}^{10}$, were prepared according to the literatures reported. Known Thio substrates $\mathbf{2} \mathbf{c a}^{6}, \mathbf{2} \mathbf{d a}^{6}$,

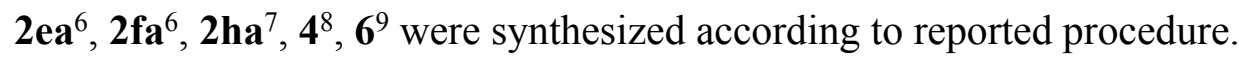

1,3,5-trimethoxy-2-vinylbenzene $\mathbf{1 f}$ ${ }_{\text {OMe }}^{1} \mathrm{H}$ NMR $\left(300 \mathrm{MHz}, \mathrm{CDCl}_{3}\right): \delta .22(\mathrm{~d}, J=6.70-6.58(\mathrm{~m}, 3 \mathrm{H}), 5.66(\mathrm{~d}, J=17.5 \mathrm{~Hz}, 1 \mathrm{H}), 3.88(\mathrm{~s}, 6 \mathrm{H}), 3.85(\mathrm{~s}, 3 \mathrm{H})$.

trimethyl((3-vinylphenyl)ethynyl)silane $\mathbf{1 m}$

TMS ${ }^{1} \mathrm{H}$ NMR (300 MHz, CDCl3): $\delta=7.52(\mathrm{~s}, 1 \mathrm{H}), 7.35(\mathrm{~d}, J=7.9$ $\mathrm{Hz}, 2 \mathrm{H}), 7.27(\mathrm{~d}, J=5.4 \mathrm{~Hz}, 1 \mathrm{H}), 6.67$ (dd, $J=17.6,10.9 \mathrm{~Hz}, 1 \mathrm{H})$, $5.75(\mathrm{~d}, J=17.9 \mathrm{~Hz}, 1 \mathrm{H}), 5.27(\mathrm{~d}, J=10.9 \mathrm{~Hz}, 1 \mathrm{H}), 0.26(\mathrm{~s}, 9 \mathrm{H})$.

4,4,5,5-tetramethyl-2-(4-vinylphenyl)-1,3,2-dioxaborolane $\mathbf{1 n}$ ${ }_{\text {PinB }}^{1} \mathrm{H}$ NMR $\left(300 \mathrm{MHz}, \mathrm{CDCl}_{3}\right): \delta=7.79(\mathrm{~d}, J=8.0 \mathrm{~Hz}, 2 \mathrm{H}), 7.42(\mathrm{~d}, J=$ $8.0 \mathrm{~Hz}, 2 \mathrm{H}), 6.74(\mathrm{dd}, J=17.6,10.9 \mathrm{~Hz}, 1 \mathrm{H}), 5.82(\mathrm{~d}, J=17.6 \mathrm{~Hz}$, $1 \mathrm{H}), 5.30(\mathrm{~d}, J=10.9 \mathrm{~Hz}, 1 \mathrm{H}), 1.36(\mathrm{~s}, 12 \mathrm{H})$.

1-(3-vinylphenyl)ethan-1-one 10<smiles>C=Cc1cccc(C(C)=O)c1</smiles>

${ }^{1} \mathrm{H}$ NMR $\left(300 \mathrm{MHz}, \mathrm{CDCl}_{3}\right): \delta=7.98(\mathrm{~s}, 1 \mathrm{H}), 7.84(\mathrm{~d}, J=7.7 \mathrm{~Hz}, 1 \mathrm{H}), 7.61$ $(\mathrm{d}, J=7.7 \mathrm{~Hz}, 1 \mathrm{H}), 7.41(\mathrm{t}, J=15.8,1 \mathrm{H}), 6.77(\mathrm{dd}, J=17.6,10.9 \mathrm{~Hz}, 1 \mathrm{H})$, $5.84(\mathrm{~d}, J=17.6 \mathrm{~Hz}, 1 \mathrm{H}), 5.33(\mathrm{~d}, J=10.9 \mathrm{~Hz}, 1 \mathrm{H}), 2.62(\mathrm{~s}, 3 \mathrm{H})$.

3-vinylpyridine 1v

${ }^{1} \mathrm{H} \mathrm{NMR}\left(300 \mathrm{MHz}, \mathrm{CDCl}_{3}\right): \delta=8.60(\mathrm{~d}, J=1.8 \mathrm{~Hz}, 1 \mathrm{H}), 8.49(\mathrm{dd}, J=4.8$, $1.3 \mathrm{~Hz}, 1 \mathrm{H}), 7.74(\mathrm{~d}, J=7.9 \mathrm{~Hz}, 1 \mathrm{H}), 7.27$ (dd, $J=8.0,4.8 \mathrm{~Hz}, 1 \mathrm{H}), 6.71$ (dd, $J=17.7,11.0 \mathrm{~Hz}, 1 \mathrm{H}), 5.85$ (d, $J=17.7 \mathrm{~Hz}, 1 \mathrm{H}), 5.39$ (d, $J=11.0 \mathrm{~Hz}, 1 \mathrm{H})$. 
4-vinylisoquinoline 1w

$\overbrace{n=1}$

${ }^{1} \mathrm{H}$ NMR $\left(300 \mathrm{MHz}, \mathrm{CDCl}_{3}\right): \delta=9.17(\mathrm{~s}, 1 \mathrm{H}), 8.63(\mathrm{~s}, 1 \mathrm{H}), 8.07(\mathrm{~d}, J=$ $8.5 \mathrm{~Hz}, 1 \mathrm{H}), 7.98(\mathrm{~d}, J=8.0 \mathrm{~Hz}, 1 \mathrm{H}), 7.73(\mathrm{dd}, J=8.4,7.1,1 \mathrm{H}), 7.62$ (dd, $J=8.0,7.1 \mathrm{~Hz}, 1 \mathrm{H}), 7.32(\mathrm{dd}, J=17.4,11.0 \mathrm{~Hz}, 1 \mathrm{H}), 5.86(\mathrm{~d}, J=17.4$

$\mathrm{Hz}, 1 \mathrm{H}), 5.57$ (d, $J=11.0 \mathrm{~Hz}, 1 \mathrm{H})$.

5-vinylpyrimidine $\mathbf{1 x}$

证 ${ }^{1} \mathrm{H} \mathrm{NMR}\left(300 \mathrm{MHz}, \mathrm{CDCl}_{3}\right): \delta=9.06(\mathrm{~s}, 1 \mathrm{H}), 8.75(\mathrm{~s}, 2 \mathrm{H}), 6.65$ (ddd, $J=$ $17.7,11.1,2.4 \mathrm{~Hz}, 1 \mathrm{H}), 5.91$ (dd, $J=17.7,2.6 \mathrm{~Hz}, 1 \mathrm{H}), 5.50$ (dd, $J=11.1$, $2.8 \mathrm{~Hz}, 1 \mathrm{H})$.

3-vinylthiophene $\mathbf{1 y}$

(1) ${ }^{1} \mathrm{HMR}\left(300 \mathrm{MHz}, \mathrm{CDCl}_{3}\right): \delta=7.33-7.22(\mathrm{~m}, 2 \mathrm{H}), 7.18(\mathrm{~s}, 1 \mathrm{H}), 6.71(\mathrm{dd}$, $J=17.5,11.8 \mathrm{~Hz}, 1 \mathrm{H}), 5.58(\mathrm{~d}, J=17.5 \mathrm{~Hz}, 1 \mathrm{H}), 5.20(\mathrm{~d}, J=10.8 \mathrm{~Hz}, 1 \mathrm{H})$.

3-bromo-2-vinylthiophene $\mathbf{1 z}$

${ }^{1} \mathrm{H} \mathrm{NMR}\left(300 \mathrm{MHz}, \mathrm{CDCl}_{3}\right): \delta=7.14(\mathrm{~d}, J=5.3 \mathrm{~Hz}, 1 \mathrm{H}), 6.94(\mathrm{~d}, J=5.3$ Hz, 1H), 6.87 (dd, $J=17.4,11.0 \mathrm{~Hz}, 1 \mathrm{H}), 5.64(\mathrm{~d}, J=17.4 \mathrm{~Hz}, 1 \mathrm{H}), 5.27$

$(\mathrm{d}, J=11.0 \mathrm{~Hz}, 1 \mathrm{H})$.

1,4-divinylbenzene 8

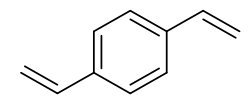

${ }^{1} \mathrm{H}$ NMR $\left(300 \mathrm{MHz}, \mathrm{CDCl}_{3}\right): \delta=7.39(\mathrm{~s}, 4 \mathrm{H}), 6.72(\mathrm{dd}, J=17.6,10.9$ $\mathrm{Hz}, 2 \mathrm{H}), 5.77$ (d, $J=18.2 \mathrm{~Hz}, 2 \mathrm{H}), 5.26$ (d, $J=10.9 \mathrm{~Hz}, 2 \mathrm{H})$.

2-mercapto-1-phenylethan-1-one 2ca

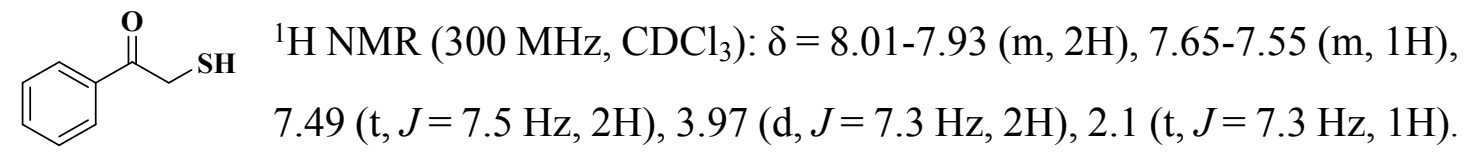


2-mercapto-1-phenylpropan-1-one 2da

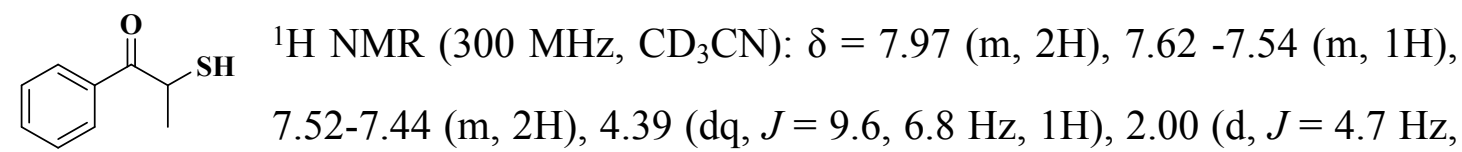
$1 \mathrm{H}), 1.63(\mathrm{~d}, J=6.8 \mathrm{~Hz}, 3 \mathrm{H})$.

2-mercapto-2-methyl-1-phenylpropan-1-one 2ea

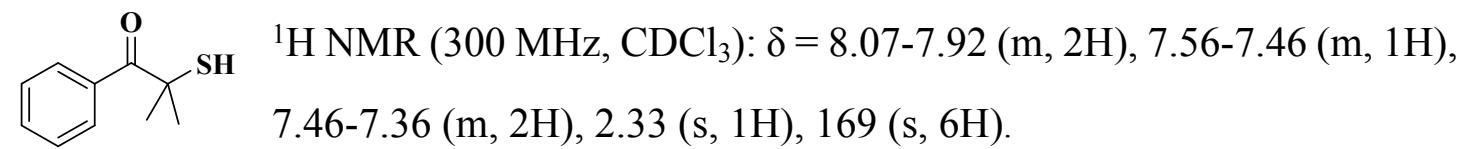

2-mercaptocyclopentan-1-one $\mathbf{2 f a}$

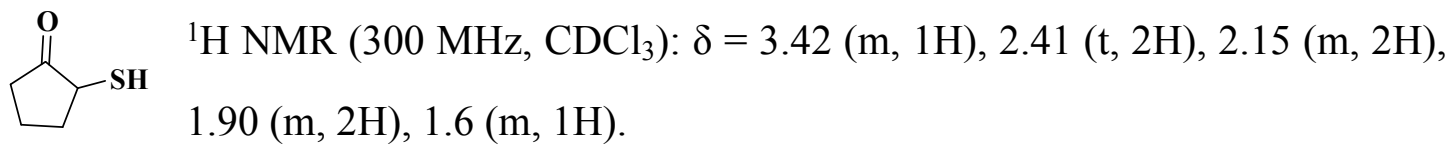

2-mercaptoacetonitrile $\mathbf{2 h a}$

${ }_{\mathbf{N C}} \widehat{ }_{\mathbf{S H}}{ }^{1} \mathrm{H}$ NMR $\left(300 \mathrm{MHz}, \mathrm{CDCl}_{3}\right): \delta=3.25(\mathrm{~d}, J=8.2 \mathrm{~Hz}, 2 \mathrm{H}), 2.37(\mathrm{t}, J=8.2$ $\mathrm{Hz}, 1 \mathrm{H})$.

methyl (2-mercaptoacetyl)- $L$-phenylalaninate 4 HS $\underbrace{\mathbf{O}}_{\mathbf{H}} \int_{\mathbf{O}}^{\mathbf{P h}}{ }_{(\mathrm{m}, 2 \mathrm{H}), 6.97(\mathrm{~d}, J=6.6,1 \mathrm{H}), 4.86(\mathrm{dt}, J=7.9,5.9 \mathrm{~Hz}, 1 \mathrm{H}), 3.74}^{{ }^{1} \mathrm{H} \text { NMR }\left(300 \mathrm{MHz}, \mathrm{CDCl}_{3}\right): \delta=7.35-7.27(\mathrm{~m}, 3 \mathrm{H}), 7.17-7.09}$ $(\mathrm{d}, J=3.0 \mathrm{~Hz}, 3 \mathrm{H}), 3.21(\mathrm{~d}, J=9.0 \mathrm{~Hz}, 2 \mathrm{H}), 3.15(\mathrm{dd}, J=9.0,5.7 \mathrm{~Hz}, 2 \mathrm{H}), 1.81(\mathrm{t}, J$ $=9.0 \mathrm{~Hz}, 1 \mathrm{H})$.

methyl (2-mercaptoacetyl)- $L$-prolinate 6<smiles>CC(=O)C1CCCN1C(=O)CS</smiles>

${ }^{1} \mathrm{H}$ NMR $(300 \mathrm{MHz}, \mathrm{MeOD}) \delta=4.43(\mathrm{dd}, J=8.7,4.0 \mathrm{~Hz}, 1 \mathrm{H}), 3.78$ $3.63(\mathrm{~m}, 5 \mathrm{H}), 3.36(\mathrm{~d}, J=6.2 \mathrm{~Hz}, 2 \mathrm{H}), 2.34-2.19(\mathrm{~m}, 1 \mathrm{H}), 2.11-1.92(\mathrm{~m}$, $3 \mathrm{H})$.

\section{General procedure for synthesis of the $2 \mathrm{ia}$}




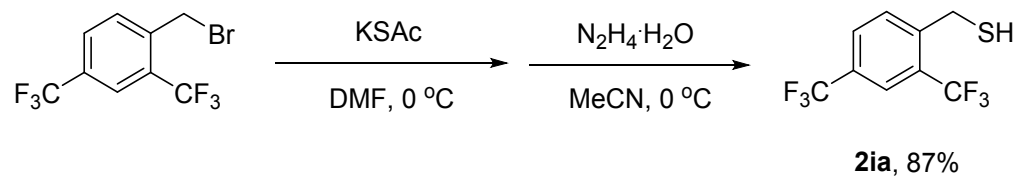

To a 1-(bromomethyl)-2,4-bis(trifluoromethyl)benzene (1 mmol, $1 N$ ) in DMF (1.0 M) was added $S$-potassium thioacetate (1.1 equiv.) at $0{ }^{\circ} \mathrm{C}$. The reaction mixture was monitored by TLC until substrate was fully consumed, quenched with water. The mixture was extracted with $\mathrm{CH}_{2} \mathrm{Cl}_{2}$, and the combined organic layer was washed water several times to remove the DMF, then washed with brine, dried over anhydrous $\mathrm{Na}_{2} \mathrm{SO}_{4}$, and concentrated in vacuo without further purification. Dissolving the product in $\mathrm{MeCN}(1.0 \mathrm{M})$ and degassed, $\mathrm{N}_{2} \mathrm{H}_{4} \cdot \mathrm{H}_{2} \mathrm{O}$ (1.0 equiv.) was added dropwise at $0{ }^{\circ} \mathrm{C}$. The reaction mixture was degassed again and stirred $2 \mathrm{~h}$. Then, the reaction was quenched with $\mathrm{HCl}$ aq. (1.0 M, $15 \mathrm{~mL})$. The mixture was extracted with $\mathrm{CH}_{2} \mathrm{Cl}_{2}$, and the combined organic layer was washed with brine $(300 \mathrm{~mL})$, dried over anhydrous $\mathrm{Na}_{2} \mathrm{SO}_{4}$, concentrated in vacuo, the crude mixture was purified by flash column chromatography to give the title compound $\mathbf{2 i a}$ as an orange oil in $87 \%$ yield.

(2,4-bis(trifluoromethyl)phenyl)methanethiol 2ia

SH Colorless oil; 87\% yield.
$\mathbf{C F}_{3} \quad$ IR (Reflection): $\tilde{v}=3201,2985,1822,1628,1587,1346,1312,1298$, 1274, 1176, 1120, 1084, 1056, 913, 863, 847, 748, 690, 668, 628; ${ }^{1} \mathrm{H}$ NMR (300 MHz, $\left.\mathrm{CDCl}_{3}\right): \delta=7.88(\mathrm{~s}, 1 \mathrm{H}), 7.80(\mathrm{~d}, J=8.3 \mathrm{~Hz}, 1 \mathrm{H}), 7.73(\mathrm{~d}, J=8.2 \mathrm{~Hz}, 1 \mathrm{H}), 3.93(\mathrm{~d}, J$ $=8.3 \mathrm{~Hz}, 2 \mathrm{H}), 1.94(\mathrm{t}, J=8.3 \mathrm{~Hz}, 1 \mathrm{H}) ;{ }^{13} \mathrm{C} \mathrm{NMR}\left(125 \mathrm{MHz}, \mathrm{CDCl}_{3}\right): \delta=144.3(\mathrm{~s}, \mathrm{~m})$, 132.3(d), 130.0(s, q, $J=33.4 \mathrm{~Hz}), 129.3(\mathrm{~d}, \mathrm{~m}), 128.5(\mathrm{~s}, \mathrm{q}, J=31.2 \mathrm{~Hz}), 124.6(\mathrm{~s}, \mathrm{~d}, J=$ $39.1 \mathrm{~Hz}), 123.5(\mathrm{~d}, \mathrm{~m}), 122.5(\mathrm{~s}, \mathrm{~d}, J=28.8 \mathrm{~Hz}), 25.2(\mathrm{t}, \mathrm{q}, J=2.1 \mathrm{~Hz}) ;{ }^{19} \mathrm{~F}$ NMR $(283$ $\mathrm{MHz}, \mathrm{CDCl}_{3}$ ): $\delta=-60.07,-62.89$; HRMS (EI) Calcd for $\mathrm{C}_{9} \mathrm{H}_{6} \mathrm{~F}_{6} \mathrm{~S}\left[\mathrm{M}^{++}\right]: 260.00889$, found: 260.01001 . 


\section{Optimization of the reaction conditions}

a \% Yields were calculated by ${ }^{1} \mathrm{H}$ NMR spectroscopy using 1,3,5-trimethoxybenzene $(0.2 \mathrm{mmol})$ as an internal standard.

Table S1. Control conditions

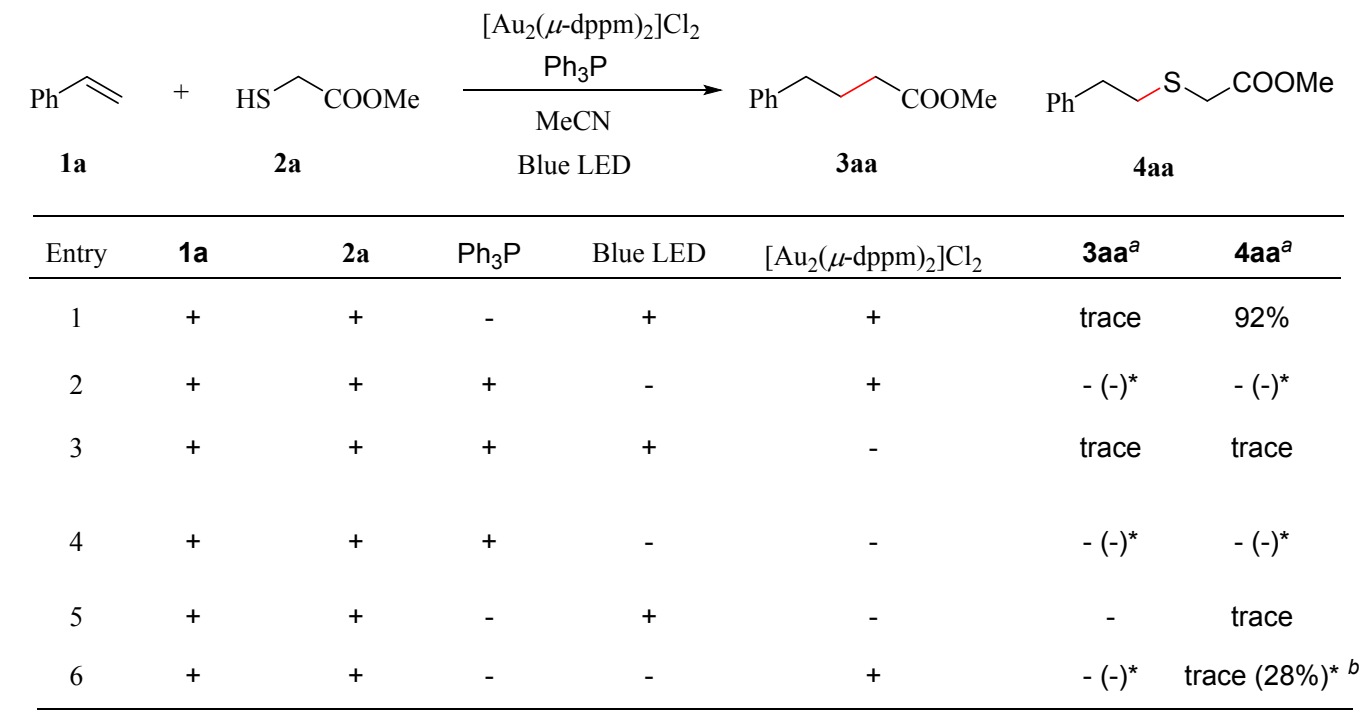

Reactions were run with $1.0 \mathrm{Ll}$ of the mixture of styrene (1.0 equiv) and mercaptan (1.2 equiv) in $\mathrm{MeCN}(0.2 \mathrm{M}),\left[\mathrm{Au}_{2}(\mu \text {-dppm })_{2}\right] \mathrm{Cl}_{2}(2.5 \mathrm{~mol} \%), \mathrm{Ph}_{3} \mathrm{P}\left(1.3\right.$ equiv) for $24 \mathrm{~h} .{ }^{*}$ Heating at $55^{\circ} \mathrm{C}$ if no reaction without light. ${ }^{b}$ See reference 14 .

Table S2. Screening of phosphorus

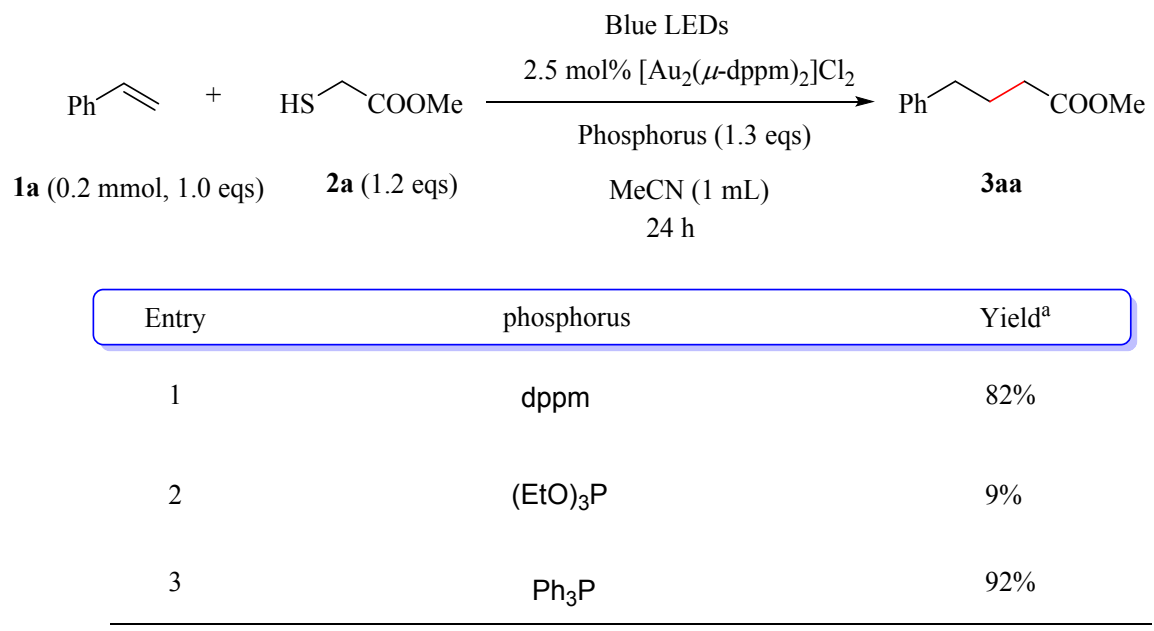


Table S3. Screening of catalyst

\begin{tabular}{|c|c|c|}
\hline & $\begin{array}{c}\text { Blue LEDs } \\
2.5 \mathrm{~mol} \% \text { catalyst }\end{array}$ & \\
\hline & $\mathrm{Ph}_{3} \mathrm{P}$ (1.3 eqs) & \\
\hline 1a ( $0.2 \mathrm{mmol}, 1.0$ eqs $)$ & $\begin{array}{l}\mathrm{MeCN}(1 \mathrm{~mL}) \\
\quad 24 \mathrm{~h}\end{array}$ & $3 \mathbf{a a}$ \\
\hline Entry & catalyst & Yield $^{\mathrm{a}}$ \\
\hline 1 & {$\left[\mathrm{Au}_{2}(\mu \text {-dppm })_{2}\right] \mathrm{Cl}_{2}$} & $92 \%$ \\
\hline 2 & {$\left[\operatorname{Ir}(\text { ppy })_{2}(\mathrm{dtbbpy})\right] \mathrm{PF}_{6}$} & $64 \%$ \\
\hline 3 & {$\left[\mathrm{Ru}(\mathrm{bpy})_{3}\right]\left(\mathrm{PF}_{6}\right)_{2}$} & $3 \%$ \\
\hline 4 & Eosin $Y$ & $20 \%$ \\
\hline 5 & Mes-Acr-Me ${ }^{+} \mathrm{BF}_{4}^{-}$ & - \\
\hline
\end{tabular}

Table S4. Concentration optimization

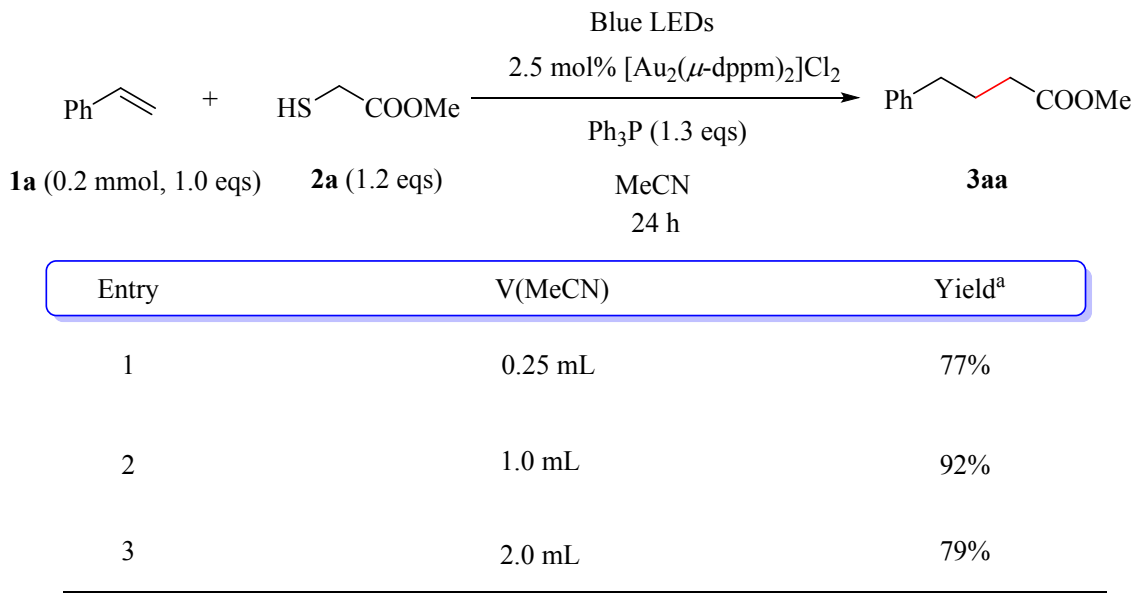

Table S5. Optimize the equivalents of mercaptan

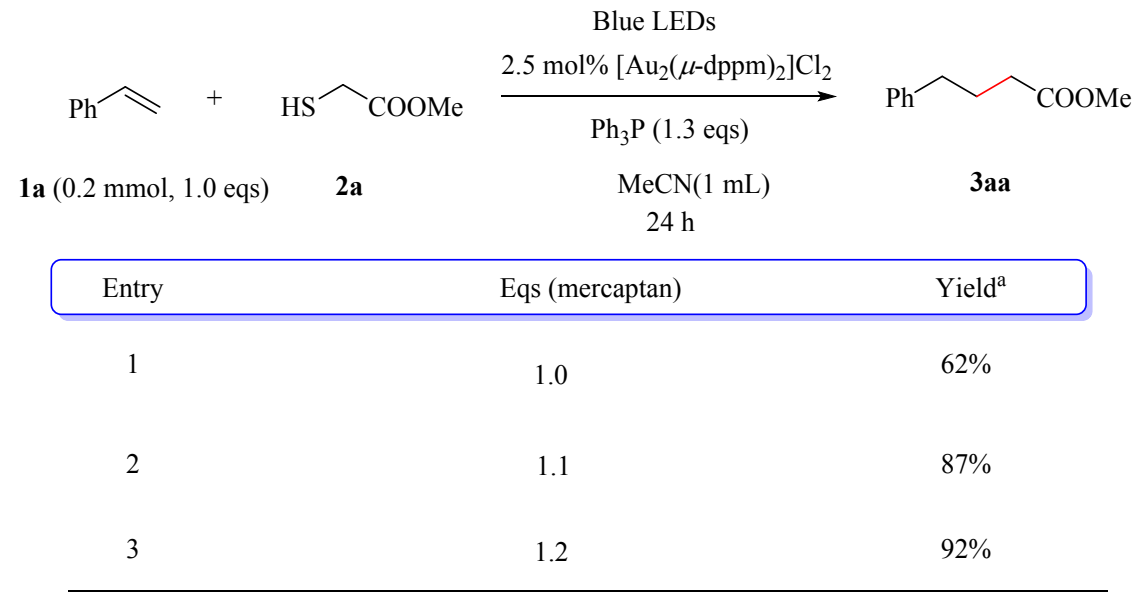


Table S6. Optimize the equivalents of $\mathrm{Ph}_{3} \mathrm{P}$

Blue LEDs

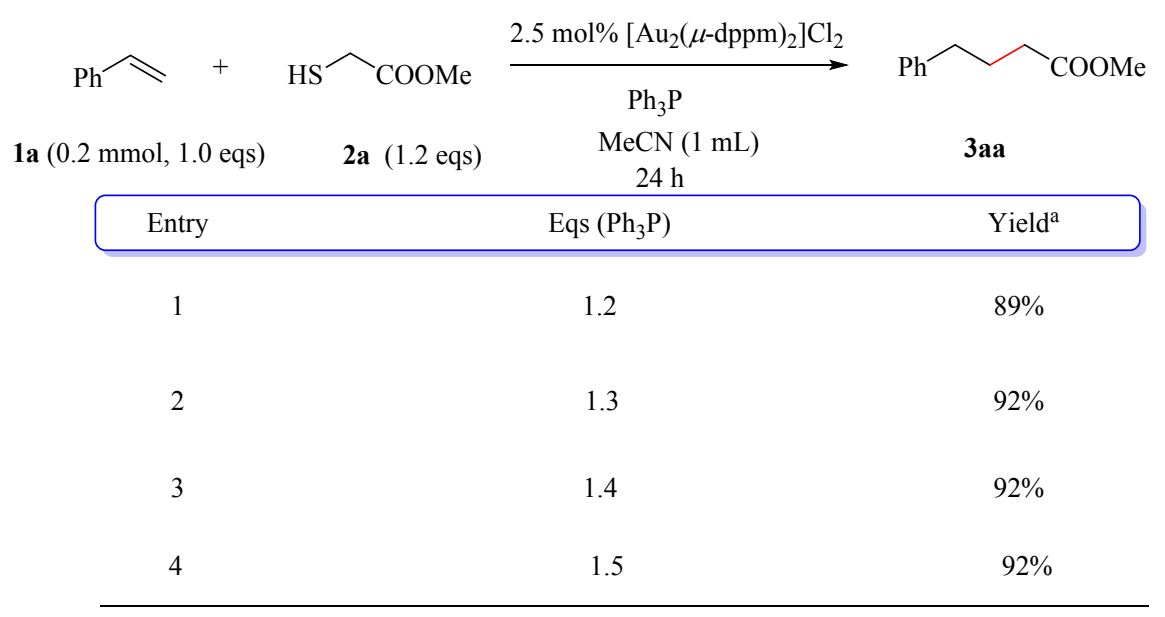

Table S7. Optimize the equivalent of $\left[\mathrm{Au}_{2}(\mu-\mathrm{dppm})_{2}\right] \mathrm{Cl}_{2}$

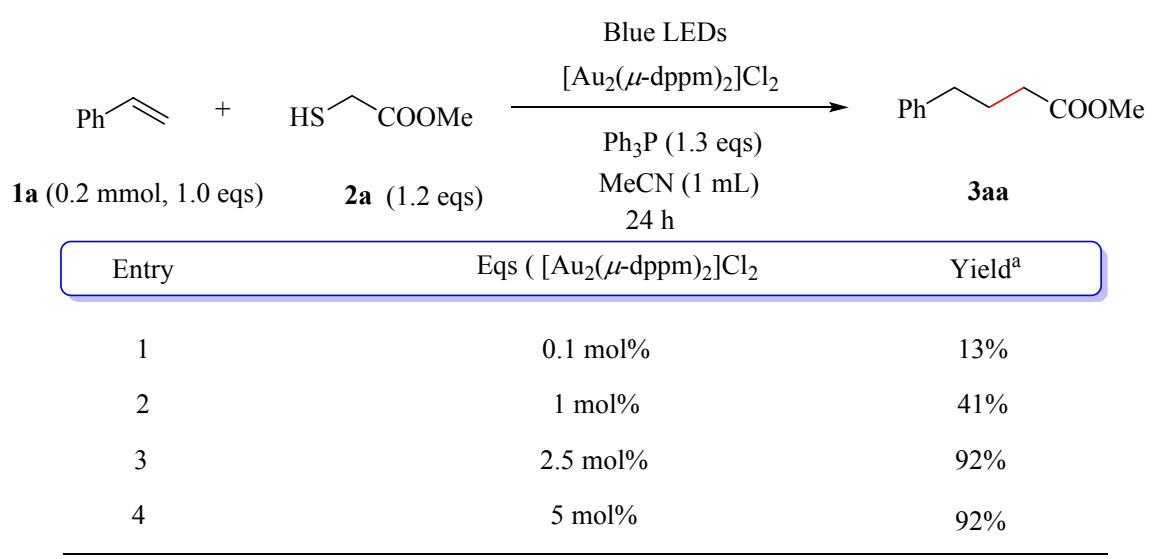

Table S8. Screening of solvent

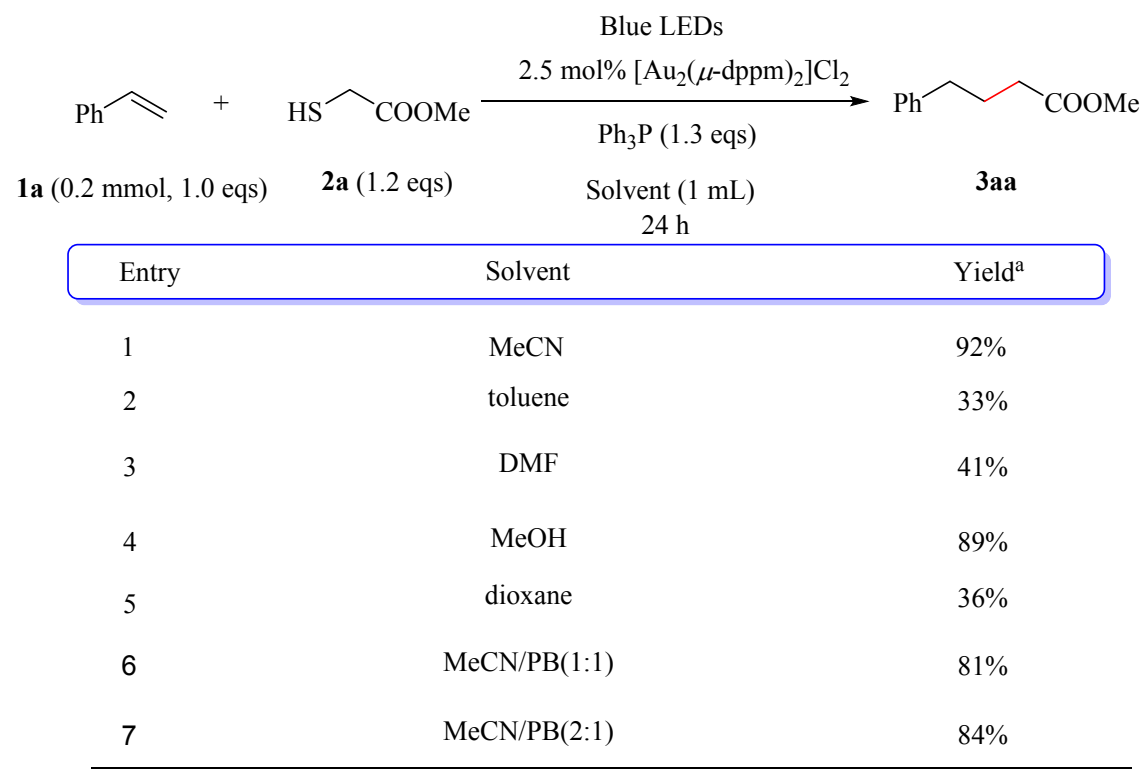




\section{General procedure for the $\mathrm{C}-\mathrm{C}$ coupling by desulfurizing gold-catalyst photoreaction}
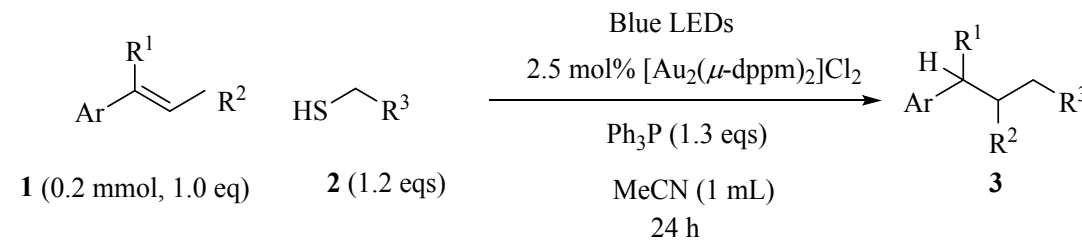

In a $10 \mathrm{~mL}$ dried pyrex screw-top reaction tube, $\left[\mathrm{Au}_{2}(\mu \text {-dppm })_{2}\right] \mathrm{Cl}_{2}(2.5 \mathrm{~mol} \%), \mathrm{Ph}_{3} \mathrm{P}$ (1.3 eqs)was dissolved in $\mathrm{MeCN}(1.0 \mathrm{~mL})$. Then the alkenes 1 (0.2 mmol, 1.0 eqs) and the mercaptans 2 (1.2 eqs) were added. The reaction mixture was then cooled to $-78{ }^{\circ} \mathrm{C}$ and degassed via vacuum evacuation ( 5 min), backfilled with nitrogen, and warmed to room temperature. This process was repeated three times. After the reaction was thoroughly degassed, irradiation of the reaction tubes was conducted with blue leds for $24 \mathrm{~h}$ and the white solid byproduct $\mathrm{Ph}_{3} \mathrm{PS}$ precipitated. When the reaction finished, the reaction mixture was diluted with DCM. After removal of the solvent in vacuo, the resulting crude products were subjected to flash column chromatography on silica gel to give desired products 3 . The products were characterized with IR, ${ }^{1} \mathrm{H} N M R,{ }^{13} \mathrm{C}$ NMR, ${ }^{19} \mathrm{~F}$ NMR and HRMS.

\section{Characterization data of the coupling products}

methyl 4-phenylbutanoate 3aa

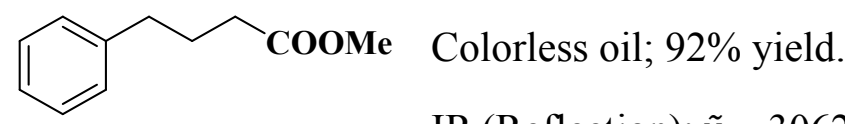

IR (Reflection): $\tilde{v}=3062,3026,2950,2858,1737,1603$ 1497,

$1454,1435,1367,1248,1203,1172,1145,1082,1030,997,886,746,700$;

${ }^{1} \mathrm{H}$ NMR $\left(300 \mathrm{MHz}, \mathrm{CDCl}_{3}\right): \delta=7.30(\mathrm{~m}, 2 \mathrm{H}), 7.21(\mathrm{~m}, 3 \mathrm{H}), 3.68(\mathrm{~s}, 3 \mathrm{H}), 2.67(\mathrm{t}, J=$ $7.6 \mathrm{~Hz}, 2 \mathrm{H}), 2.35(\mathrm{t}, J=7.5 \mathrm{~Hz}, 2 \mathrm{H}), 2.03-1.92(\mathrm{~m}, 2 \mathrm{H}) .{ }^{13} \mathrm{C} \mathrm{NMR}\left(125 \mathrm{MHz}, \mathrm{CDCl}_{3}\right)$ : $\delta=174.1(\mathrm{~s}), 141.5(\mathrm{~s}), 128.6(\mathrm{~d}), 128.5(\mathrm{~d}), 126.1(\mathrm{~d})$, 51.6(q), 35.3(t), 33.5(t), 26.6(t); HRMS (EI) Calcd for $\mathrm{C}_{11} \mathrm{H}_{14} \mathrm{O}_{2}\left[\mathrm{M}^{\bullet+}\right]$ : 178.09883 , found: 178.10109 . 
methyl 4-( $p$-tolyl)butanoate 3ab

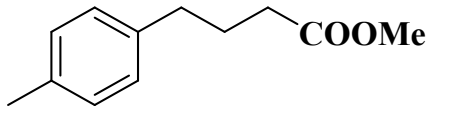

Colorless oil; $86 \%$ yield.

IR (Reflection): $\tilde{v}=3019,3004,2950,2925,2862,1899$, $1738,1515,1436,1366,1324,1305,1247,1202$, 1172, 1147, 1046, 1021, 999, 887, 806; ${ }^{1} \mathrm{H}$ NMR (500 MHz, $\mathrm{CDCl}_{3}$ ): $\delta=7.09$ (dd, $\left.J=14.7,8.1 \mathrm{~Hz}, 4 \mathrm{H}\right), 3.67(\mathrm{~s}, 3 \mathrm{H})$, $2.62(\mathrm{t}, J=7.6 \mathrm{~Hz}, 2 \mathrm{H}), 2.36-2.31(\mathrm{~m}, 5 \mathrm{H}), 1.98-1.91(\mathrm{~m}, 2 \mathrm{H}) .{ }^{13} \mathrm{C} \mathrm{NMR}(125 \mathrm{MHz}$, $\left.\mathrm{CDCl}_{3}\right): \delta=174.1(\mathrm{~s}), 138.4(\mathrm{~s}), 135.5(\mathrm{~s}), 129.2(\mathrm{~d}), 128.5(\mathrm{~d}), 51.6(\mathrm{q}), 34.8(\mathrm{t}), 33.5(\mathrm{t})$, 26.7(t), 21.1(q); HRMS (EI) Calcd for $\mathrm{C}_{12} \mathrm{H}_{16} \mathrm{O}_{2}\left[\mathrm{M}^{\cdot+}\right]$ : 192.11448, found: 192.11446 .

methyl 4-(o-tolyl)butanoate 3ac

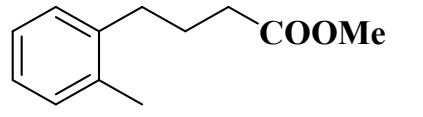

Colorless oil; $87 \%$ yield.

IR (Reflection): $\tilde{v}=3019,3005,2951,2925,2862,1900$, $1738,1517,1436,1363,1320,1305,1249,1201,1172,1144,1046,1021,1000,884$; ${ }^{1} \mathrm{H} \operatorname{NMR}\left(500 \mathrm{MHz}, \mathrm{CDCl}_{3}\right): \delta=7.17-7.09(\mathrm{~m}, 4 \mathrm{H}), 3.69(\mathrm{~s}, 3 \mathrm{H}), 2.65(\mathrm{t}, J=7.6 \mathrm{~Hz}$ 2H), 2.39 (t, $J=7.4 \mathrm{~Hz}, 2 \mathrm{H}), 2.33(\mathrm{~s}, 3 \mathrm{H}), 1.96-1.90(\mathrm{~m}, 2 \mathrm{H}) .{ }^{13} \mathrm{C}$ NMR (125 MHz, $\left.\mathrm{CDCl}_{3}\right): \delta=174.1(\mathrm{~s}), 139.7(\mathrm{~s}), 136.1(\mathrm{~s}), 130.4(\mathrm{~d}), 129.1(\mathrm{~d}), 126.2(\mathrm{~d}), 126.1(\mathrm{~d}), 51.6(\mathrm{q})$, 33.8(t), 32.7(t), 25.4(t), 19.3(q); HRMS (EI) Calcd for $\mathrm{C}_{12} \mathrm{H}_{16} \mathrm{O}_{2}\left[\mathrm{M}^{++}\right]$: 192.11448, found: 192.11398 .

methyl 4-mesitylbutanoate 3ad

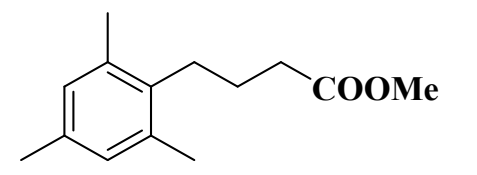

Colorless oil; $87 \%$ yield.

IR (Reflection): $\tilde{v}=2951,2921,1741,1614,1579,1485$, $1454,1436,1362,1249,1200,1177,1149,1073,1014852$, 720; ${ }^{1} \mathrm{H}$ NMR (300 MHz, $\left.\mathrm{CDCl}_{3}\right): \delta=6.75(\mathrm{~s}, 2 \mathrm{H}), 3.62(\mathrm{~s}, 3 \mathrm{H}), 2.58-2.49(\mathrm{~m}, 2 \mathrm{H})$, $2.35(\mathrm{t}, J=7.2 \mathrm{~Hz}, 2 \mathrm{H}), 2.21(\mathrm{~s}, 6 \mathrm{H}), 2.17(\mathrm{~s}, 3 \mathrm{H}), 1.77-1.64(\mathrm{~m}, 2 \mathrm{H}) ;{ }^{13} \mathrm{C}$ NMR $(125$ $\left.\mathrm{MHz}, \mathrm{CDCl}_{3}\right): \delta=174.1(\mathrm{~s}), 136.2(\mathrm{~s}), 135.4(\mathrm{~s}), 135.3(\mathrm{~s}), 129.0(\mathrm{~d}), 51.7(\mathrm{q}), 34.3(\mathrm{t})$, 28.9(t), 24.5(t), 20.9(q), 19.8(q); HRMS (EI) Calcd for $\mathrm{C}_{14} \mathrm{H}_{20} \mathrm{O}_{2}\left[\mathrm{M}^{\bullet+}\right]:$ 220.14578, found: 220.14512 . 
methyl 4-(o-tolyl)butanoate 3ae

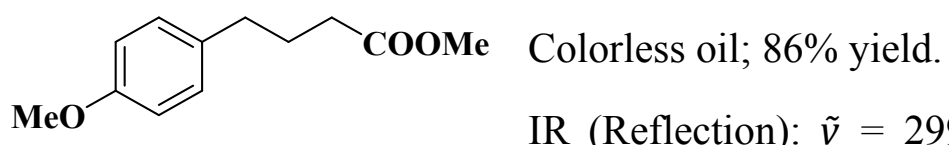

IR (Reflection): $\tilde{v}=2997,2950,2836,2059,1882$, $1734,1612,1583,1511,1437,1365,1300,1244,1175,1144,1108,1033,886,810$ 749, 698; ${ }^{1} \mathrm{H}$ NMR $\left(500 \mathrm{MHz}, \mathrm{CDCl}_{3}\right): \delta=7.10(\mathrm{~d}, J=8.7 \mathrm{~Hz}, 2 \mathrm{H}), 6.83(\mathrm{~d}, J=8.7$ $\mathrm{Hz}, 2 \mathrm{H}), 3.79$ (s, 3H), $3.67(\mathrm{~s}, 3 \mathrm{H}), 2.60(\mathrm{t}, J=7.6 \mathrm{~Hz}, 2 \mathrm{H}), 2.33(\mathrm{t}, J=7.5 \mathrm{~Hz}, 2 \mathrm{H})$, 1.97-1.89 (m, 2H). ${ }^{13} \mathrm{C}$ NMR (125 MHz, $\left.\mathrm{CDCl}_{3}\right): \delta=174.1(\mathrm{~s}), 158.0(\mathrm{~s}), 133.6(\mathrm{~s})$, 129.5(d), 113.9(d), 55.4(q), 51.6(q), 34.3(t), 33.5(t), 26.8(t). HRMS (EI) Calcd for $\mathrm{C}_{12} \mathrm{H}_{16} \mathrm{O}_{3}\left[\mathrm{M}^{\bullet+}\right]$ : 208.10940, found: 208.10871.

methyl 4-(2,4,6-trimethoxyphenyl)butanoate 3af<smiles>COc1cc(OC)c(CCCC(C)=O)c(OC)c1</smiles>

Colorless oil; $88 \%$ yield.

IR (Reflection): $\tilde{v}=2949,2839,2594,2011,1729$, $1586,1505,1454,1418,1346,1230,1116,1048$, 1007,923, 825, 776, 745, 664; ${ }^{1} \mathrm{H}$ NMR (500 MHz, $\left.\mathrm{CDCl}_{3}\right): \delta=6.40$ (s, 2H), 3.85 (s, 6H), $3.82(\mathrm{~s}, 3 \mathrm{H}), 3.68(\mathrm{~s}, 3 \mathrm{H}), 2.60(\mathrm{t}, J=7.6 \mathrm{~Hz}, 2 \mathrm{H}), 2.35$ (t, $J=7.4 \mathrm{~Hz}, 2 \mathrm{H}), 1.99$ $1.91(\mathrm{~m}, 2 \mathrm{H}) ;{ }^{13} \mathrm{C} \mathrm{NMR}\left(126 \mathrm{MHz}, \mathrm{CDCl}_{3}\right): \delta=174.1(\mathrm{~s}), 153.2(\mathrm{~s}), 137.3(\mathrm{~s}), 136.3(\mathrm{~s})$, 105.5(d), 61.0(q), 56.2(q), 51.7(q), 35.7(t), 33.5(t), 26.7(t); HRMS (EI) Calcd for $\mathrm{C}_{14} \mathrm{H}_{20} \mathrm{O}_{5}\left[\mathrm{M}^{\cdot+}\right]: 268.13053$, found: 268.13159 .

methyl 4-(4-(tert-butyl)phenyl)butanoate 3ag

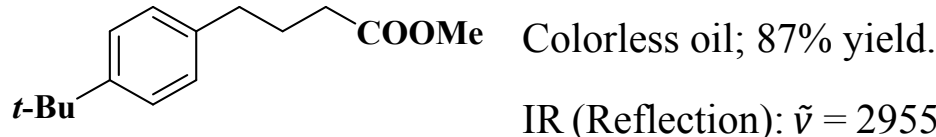
IR (Reflection): $\tilde{v}=2955,2873,2590,2018,1745,1566$, $152,1454,1411,1370,1233,1169,1078,929,855,770,755,624$

${ }^{1} \mathrm{H}$ NMR $\left(500 \mathrm{MHz}, \mathrm{CDCl}_{3}\right): \delta=7.23(\mathrm{~d}, J=8.2 \mathrm{~Hz}, 2 \mathrm{H}), 7.04(\mathrm{~d}, J=8.2 \mathrm{~Hz}, 2 \mathrm{H})$, $3.59(\mathrm{~s}, 3 \mathrm{H}), 2.56(\mathrm{t}, J=7.6 \mathrm{~Hz}, 2 \mathrm{H}), 2.27(\mathrm{t}, J=7.5 \mathrm{~Hz}, 2 \mathrm{H}), 1.92-1.84(\mathrm{~m}, 2 \mathrm{H}), 1.24$ (s, 9H); ${ }^{13} \mathrm{C}$ NMR (125 MHz, $\left.\mathrm{CDCl}_{3}\right): \delta=174.2(\mathrm{~s}), 148.9(\mathrm{~s}), 138.4(\mathrm{~s}), 128.3(\mathrm{~d})$, 125.4(d), 51.6(q), 34.7(t), 34.50(s), 33.6(t), 31.54(q), 26.59(t); HRMS (EI) Calcd for 
$\mathrm{C}_{15} \mathrm{H}_{22} \mathrm{O}_{2}\left[\mathrm{M}^{+}\right]$: 234.16143, found: 234.16084 .

methyl 4-(4-fluorophenyl)butanoate 3ah

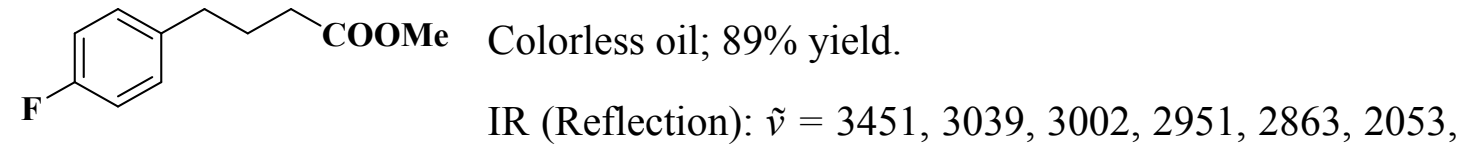
1888, 1730, 1601, 1507, 1436, 1366, 1215, 1147, 1097, 1015, 888, 821, 764, 702;

${ }^{1} \mathrm{H}$ NMR (500 MHz, $\left.\mathrm{CDCl}_{3}\right): \delta=7.16-7.10(\mathrm{~m}, 2 \mathrm{H}), 7.00-6.94(\mathrm{~m}, 2 \mathrm{H}), 3.67(\mathrm{~s}, 3 \mathrm{H})$, $2.62(\mathrm{t}, J=7.6 \mathrm{~Hz}, 2 \mathrm{H}), 2.32$ (t, $J=7.4 \mathrm{~Hz}, 2 \mathrm{H}), 1.97-1.90(\mathrm{~m}, 2 \mathrm{H})$;

${ }^{13} \mathrm{C}$ NMR (125 MHz, $\left.\mathrm{CDCl}_{3}\right): \delta=174.0(\mathrm{~s}), 161.5(\mathrm{~s}, \mathrm{~d}, J=242.1 \mathrm{~Hz}), 137.1(\mathrm{~s}, \mathrm{~d}, J=$ $3.2 \mathrm{~Hz}), 130.0(\mathrm{~d}, \mathrm{~d}, J=7.8 \mathrm{~Hz}), 115.3(\mathrm{~d}, \mathrm{~d}, J=21.1 \mathrm{~Hz}), 51.7(\mathrm{q}), 34.4(\mathrm{t}), 33.4(\mathrm{t})$, 26.7(t); ${ }^{19} \mathrm{~F}$ NMR (283 MHz, $\mathrm{CDCl}_{3}$ ): $\delta=-117.54$; HRMS (EI) Calcd for $\mathrm{C}_{11} \mathrm{H}_{13} \mathrm{O}_{2} \mathrm{~F}$ $\left[\mathrm{M}^{\circ}\right]$ : 196.08941 , found: 196.09046 .

methyl 4-(3-bromophenyl)butanoate 3ai

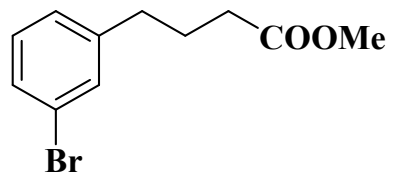

Colorless oil; $90 \%$ yield.

IR (Reflection): $\tilde{v}=2950,2862,1737,1595,1567,1474$, $1435,1247,1202,1170,1147,1093,1071,997,882,835,780$, 693, 668; ${ }^{1} \mathrm{H}$ NMR $\left(500 \mathrm{MHz}, \mathrm{CDCl}_{3}\right): \delta=7.35-7.31(\mathrm{~m}, 2 \mathrm{H}), 7.15(\mathrm{t}, J=7.9 \mathrm{~Hz}, 1 \mathrm{H})$, 7.12-7.09 (m, 1H), 3.67 (s, 3H), $2.63(\mathrm{t}, J=7.6 \mathrm{~Hz}, 2 \mathrm{H}), 2.33(\mathrm{t}, J=7.4 \mathrm{~Hz}, 2 \mathrm{H}), 2.00-$ $1.90(\mathrm{~m}, 2 \mathrm{H}) ;{ }^{13} \mathrm{C}$ NMR $\left(125 \mathrm{MHz}, \mathrm{CDCl}_{3}\right): \delta=173.8(\mathrm{~s}), 143.9(\mathrm{~s}), 131.6(\mathrm{~d}), 130.1(\mathrm{~d})$, 129.3(d), 127.3(d), 122.6(s), 51.7(q), 34.9(t), 33.4(t), 26.4(t); HRMS (EI) Calcd for $\mathrm{C}_{11} \mathrm{H}_{13} \mathrm{O}_{2} \mathrm{Br}\left[\mathrm{M}^{\circ}\right]$ : 256.00934, found: 256.00923 .

methyl 4-(2-bromophenyl)butanoate 3aj<smiles>CC(=O)OCCCc1ccccc1Br</smiles>

IR (Reflection): $\tilde{v}=3057,2950,2867,1733,1593,1567$, $1471,1437,1367,1248,1201,1173,1151,1116,1021,887,751,657 ;{ }^{1} \mathrm{H}$ NMR (500 $\left.\mathrm{MHz}, \mathrm{CDCl}_{3}\right): \delta=7.55-7.51(\mathrm{~m}, 1 \mathrm{H}), 7.26-7.19(\mathrm{~m}, 2 \mathrm{H}), 7.09-7.04(\mathrm{~m}, 2.5 \mathrm{~Hz}, 1 \mathrm{H})$, 
$3.68(\mathrm{~s}, 3 \mathrm{H}), 2.78(\mathrm{t}, J=7.6 \mathrm{~Hz}, 2 \mathrm{H}), 2.38(\mathrm{t}, J=7.5 \mathrm{~Hz}, 2 \mathrm{H}), 2.01-1.92(\mathrm{~m}, 2 \mathrm{H}) ;{ }^{13} \mathrm{C}$ NMR (125 MHz, $\mathrm{CDCl}_{3}$ ): $\delta=173.9(\mathrm{~s}), 140.8(\mathrm{~s}), 133.0(\mathrm{~d}), 130.6(\mathrm{~d}), 127.9(\mathrm{~d}), 127.6(\mathrm{~d})$, 124.6(s), 51.7(q), 35.4(t), 33.5(t), 25.1(t); HRMS (EI) Calcd for $\mathrm{C}_{11} \mathrm{H}_{13} \mathrm{O}_{2} \mathrm{Br}\left[\mathrm{M}^{\bullet+}\right]$ : 256.00934, found: 256.01040 .

methyl 4-(4-chlorophenyl)butanoate 3ak<smiles>COC(=O)CCCc1ccc(Cl)cc1</smiles>

IR (Reflection): $\tilde{v}=3026,2950,2862,1897,1733,1597$, $1492,1436,1408,1365,1320,1301,1247,1202,1172,1147,1091,1014,834$

800, 715, 660, 626; ${ }^{1} \mathrm{H} \mathrm{NMR}\left(500 \mathrm{MHz}, \mathrm{CDCl}_{3}\right):=\delta 7.25(\mathrm{~d}, J=8.3 \mathrm{~Hz}, 2 \mathrm{H}), 7.11(\mathrm{~d}$, $J=8.3 \mathrm{~Hz}, 2 \mathrm{H}), 3.67$ (s, 3H), 2.62 (t, $J=7.6 \mathrm{~Hz}, 2 \mathrm{H}), 2.32$ (t, $J=7.4 \mathrm{~Hz}, 2 \mathrm{H}), 1.98$ $1.89(\mathrm{~m}, 2 \mathrm{H}) ;{ }^{13} \mathrm{C} \mathrm{NMR}\left(125 \mathrm{MHz}, \mathrm{CDCl}_{3}\right): \delta=173.9(\mathrm{~s}), 139.9(\mathrm{~s}), 131.9(\mathrm{~s}), 130.0(\mathrm{~d})$, 128.6(d), 51.7(q), 34.6(t), 33.4(t), 26.5(t); HRMS (EI) Calcd for $\mathrm{C}_{11} \mathrm{H}_{13} \mathrm{O}_{2} \mathrm{Cl}\left[\mathrm{M}^{\bullet+}\right]$ : 212.05986, found: 212.05903 .

methyl 4-(4-(trifluoromethyl)phenyl)butanoate 3al<smiles>COC(=O)CCCc1ccc(C(F)(F)F)cc1</smiles>

IR (Reflection): $\tilde{v}=2954,2867,2646,1921,1735,1619$, $1585,1438,1419,1367,1333,1251,1158,1068,1019,846,818,732,636,612 ;{ }^{1} \mathrm{H}$ NMR (300 MHz, $\left.\mathrm{CDCl}_{3}\right): \delta=7.54(\mathrm{~d}, J=8.1 \mathrm{~Hz}, 2 \mathrm{H}), 7.29(\mathrm{~d}, J=8.0 \mathrm{~Hz}, 2 \mathrm{H}), 3.67$ (s, 3H), $2.71(\mathrm{t}, J=7.6 \mathrm{~Hz}, 2 \mathrm{H}), 2.34$ (t, $J=7.4 \mathrm{~Hz}, 2 \mathrm{H}), 2.02-1.92(\mathrm{~m}, 2 \mathrm{H})$; ${ }^{13} \mathrm{C} \mathrm{NMR}\left(125 \mathrm{MHz}, \mathrm{CDCl}_{3}\right): \delta=173.8(\mathrm{~s}), 145.6(\mathrm{~s}), 128.9(\mathrm{~d}), 128.6(\mathrm{~s}, \mathrm{~d}, J=32.1 \mathrm{~Hz})$, 125.5 (d, q, $J=3.8 \mathrm{~Hz}), 123.4$ (s), 51.7(q), 35.0(t), 33.4(t), 26.3(t); ${ }^{19} \mathrm{~F}$ NMR (283 MHz, $\mathrm{CDCl}_{3}$ ): $\delta=-62.37$; HRMS (EI) Calcd for $\mathrm{C}_{12} \mathrm{H}_{13} \mathrm{O}_{2} \mathrm{~F}_{3}\left[\mathrm{M}^{\bullet+}\right]$ : 246.08622, found: 246.08478 . 
methyl 4-(3-((trimethylsilyl)ethynyl)phenyl)butanoate 3am

TMS<smiles>CC#Cc1cccc(CCCC(=O)OC)c1</smiles>

Colorless oil; $84 \%$ yield

IR (Reflection): $\tilde{v}=3024,2955,2862,2153,1740$, $1600,1578,1481,1436,1367,1317,1250,1206$, 1171, 1148, 1087, 1048, 1001, 939, 845, 796, 760, 697, 647; ${ }^{1} \mathrm{H}$ NMR (500 MHz, $\left.\mathrm{CDCl}_{3}\right): \delta=7.32-7.28(\mathrm{~m}, 2 \mathrm{H}), 7.21(\mathrm{t}, J=7.8 \mathrm{~Hz}, 1 \mathrm{H}), 7.12(\mathrm{~d}, J=7.7 \mathrm{~Hz}, 1 \mathrm{H}), 3.66$ (s, 3H), $2.61(\mathrm{t}, J=7.6 \mathrm{~Hz}, 2 \mathrm{H}), 2.31(\mathrm{t}, J=7.5 \mathrm{~Hz}, 2 \mathrm{H}), 1.98-1.89(\mathrm{~m}, 2 \mathrm{H}), 0.25(\mathrm{~s}$, 9H); ${ }^{13} \mathrm{C}$ NMR (125 MHz, $\mathrm{CDCl}_{3}$ ): $\delta=173.9(\mathrm{~s}), 141.5(\mathrm{~s}), 132.1(\mathrm{~d}), 129.8(\mathrm{~d}), 129.0(\mathrm{~d})$, 128.4(d), 123.2(s), 105.3(s), 94.0(s), 51.7(q), 35.0(t), 33.4(t), 26.4(t), 0.13(q); HRMS (EI) Calcd for $\mathrm{C}_{16} \mathrm{H}_{22} \mathrm{O}_{2} \mathrm{Si}\left[\mathrm{M}^{\cdot+}\right]$ : 274.13836, found: 274.13967 .

methyl 4-(4-(4,4,5,5-tetramethyl-1,3,2-dioxaborolan-2-yl)phenyl)butanoate 3an

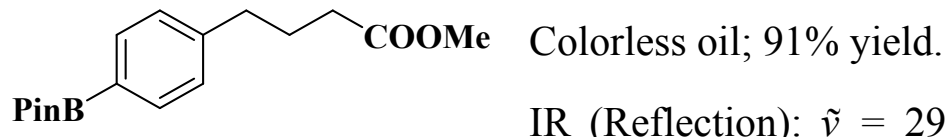
IR (Reflection): $\tilde{v}=2978,2951,2865,1740,1612$, $1561,1518,1437,1399,1365,1321,1271,1214,1146,1090,1021,963,859,824$ 740, 659; ${ }^{1} \mathrm{H}$ NMR $\left(500 \mathrm{MHz}, \mathrm{CDCl}_{3}\right): \delta=7.74(\mathrm{~d}, J=7.9 \mathrm{~Hz}, 2 \mathrm{H}), 7.19(\mathrm{~d}, J=7.9$ Hz, 2H), $3.66(\mathrm{~s}, 3 \mathrm{H}), 2.66(\mathrm{t}, J=7.6 \mathrm{~Hz}, 2 \mathrm{H}), 2.32(\mathrm{t}, J=7.5 \mathrm{~Hz}, 2 \mathrm{H}), 1.99-1.93(\mathrm{~m}$, 2H), $1.34(\mathrm{~s}, 12 \mathrm{H}) ;{ }^{13} \mathrm{C}$ NMR (125 MHz, $\left.\mathrm{CDCl}_{3}\right): \delta=174.0(\mathrm{~s}), 144.9(\mathrm{~s}), 135.1(\mathrm{~d})$, 128.1(d), 83.8(s), 51.6(q), 35.4(t), 33.5(t), 26.4(t), 25.0(q); HRMS (EI) Calcd for $\mathrm{C}_{17} \mathrm{H}_{25} \mathrm{O}_{4} \mathrm{~B}\left[\mathrm{M}^{\cdot+}\right]:$ 304.18459, found: 304.18570 .

methyl 4-(3-acetylphenyl)butanoate 3ao

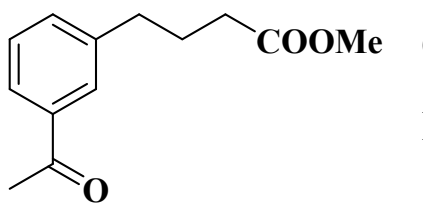

Colorless oil; $95 \%$ yield.

IR (Reflection): $\tilde{v}=3627,3350,3001,2951,2862,2063$, $1732,1682,1601,1584,1485,1436,1358,1269,1192,1147$,

1083, 1047, 999, 958, 891, 863, 796, 715, 693; ${ }^{1} \mathrm{H}$ NMR (300 MHz, $\left.\mathrm{CDCl}_{3}\right): \delta=7.76-$ $7.68(\mathrm{~m}, 2 \mathrm{H}), 7.36-7.28(\mathrm{~m}, 2 \mathrm{H}), 3.60(\mathrm{~s}, 3 \mathrm{H}), 2.64(\mathrm{t}, J=7.6 \mathrm{~Hz}, 2 \mathrm{H}), 2.53(\mathrm{~s}, 3 \mathrm{H})$, $2.27(\mathrm{t}, J=7.4 \mathrm{~Hz}, 2 \mathrm{H}), 1.96-1.86(\mathrm{~m}, 2 \mathrm{H}) .{ }^{13} \mathrm{C} \mathrm{NMR}\left(125 \mathrm{MHz}, \mathrm{CDCl}_{3}\right): \delta=198.4(\mathrm{~s})$, 
173.8(s), 142.1(s), 137.5(s), 133.4(d), 128.8(d), 128.3(d), 126.4(d), 51.7(q), 35.1(t), 33.4(t), 26.8(q), 26.5(t); HRMS (EI) Calcd for $\mathrm{C}_{13} \mathrm{H}_{16} \mathrm{O}_{3}\left[\mathrm{M}^{+}\right]$: 220.10940, found: 220.10854 .

methyl 4-(naphthalen-2-yl)butanoate 3ap

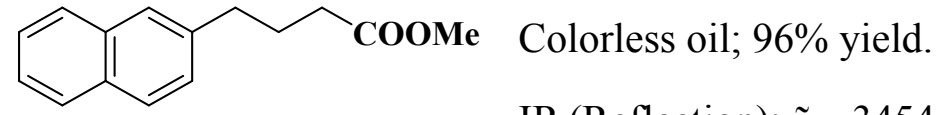

IR (Reflection): $\tilde{v}=3454,3051,3020,2949,2857,1920$, 1730, 1632, 1600, 1507, 1435, 1365, 1335, 1310, 1245, 1198, 1159, 1052, 1017 893, 853, 817, 746, 648, 619; ${ }^{1} \mathrm{H}$ NMR (500 MHz, $\left.\mathrm{CDCl}_{3}\right): \delta=7.81(\mathrm{~d}, J=7.5 \mathrm{~Hz}$, $1 \mathrm{H}), 7.78(\mathrm{~d}, J=8.5 \mathrm{~Hz}, 2 \mathrm{H}), 7.62(\mathrm{~s}, 1 \mathrm{H}), 7.48-7.40(\mathrm{~m}, 2 \mathrm{H}), 7.33(\mathrm{dd}, J=8.4,1.6 \mathrm{~Hz}$, 1H), 3.67 (s, 3H), 2.83 (t, $J=7.6 \mathrm{~Hz}, 2 \mathrm{H}), 2.38$ (t, $J=7.5 \mathrm{~Hz}, 2 \mathrm{H}), 2.10-2.01$ (m, $2 \mathrm{H})$; ${ }^{13} \mathrm{C}$ NMR (125 MHz, $\left.\mathrm{CDCl}_{3}\right): \delta=174.1(\mathrm{~s}), 139.0(\mathrm{~s}), 133.7(\mathrm{~s}), 132.2(\mathrm{~s}), 128.1(\mathrm{~d})$, 127.7(d), 127.6(d), 127.4(d), 126.7(d), 126.1(d), 125.4(d), 51.7(q), 35.4(t), 33.5(t), 26.5(t); HRMS (EI) Calcd for $\mathrm{C}_{15} \mathrm{H}_{16} \mathrm{O}_{2}\left[\mathrm{M}^{\cdot+}\right]$ : 228.11448, found: 228.11391 .

methyl 3-methyl-4-phenylbutanoate 3aq COOMe Colorless oil; 84\% yield. IR (Reflection): $\tilde{v}=3063,3027,2955,2928,2873,2852$, 1741, 1603, 1496, 1454, 1436, 1366, 1265, 1209, 1161, 1088, 1053, 1030, 1009, 911 $882,843,743,701 ;{ }^{1} \mathrm{H}$ NMR $\left(500 \mathrm{MHz}, \mathrm{CDCl}_{3}\right): \delta=7.28(\mathrm{t}, J=7.4 \mathrm{~Hz}, 2 \mathrm{H}), 7.22-$ $7.14(\mathrm{~m}, 3 \mathrm{H}), 3.65$ (s, 3H), $2.62(\mathrm{dd}, J=13.4,6.7 \mathrm{~Hz}, 1 \mathrm{H}), 2.51(\mathrm{dd}, J=13.4,7.4 \mathrm{~Hz}$, 1H), 2.37-2.24 (m, 2H), 2.15 (dd, $J=14.4,7.7 \mathrm{~Hz}, 1 \mathrm{H}), 0.95$ (d, $J=6.6 \mathrm{~Hz}, 3 \mathrm{H}) ;{ }^{13} \mathrm{C}$ $\operatorname{NMR}\left(125 \mathrm{MHz}, \mathrm{CDCl}_{3}\right): \delta=173.7(\mathrm{~s}), 140.4(\mathrm{~s}), 129.4(\mathrm{~d}), 128.4(\mathrm{~d}), 126.2(\mathrm{~d}), 51.5(\mathrm{q})$, 43.2(t), 41.0(t), 32.4(d), 19.8(q); HRMS (EI) Calcd for $\mathrm{C}_{12} \mathrm{H}_{16} \mathrm{O}_{2}\left[\mathrm{M}^{*+}\right]$ : 192.11448, found: 192.11360 . 
methyl 4-phenylpentanoate 3ar<smiles>COC(=O)CCC(C)c1ccccc1</smiles>

Colorless oil; $86 \%$ yield.

IR (Reflection): $\tilde{v}=3062,3027,2957,2930,2873,1949$, $1876,1739,1603,1494,1453,1436,1378,1326,1256,1209$, $1166,1107,1072,1021,986,911,837,763,701 ;{ }^{1} \mathrm{H} \mathrm{NMR}\left(500 \mathrm{MHz}, \mathrm{CDCl}_{3}\right): \delta=7.30$ $(\mathrm{t}, J=7.6 \mathrm{~Hz}, 2 \mathrm{H}), 7.22-7.16(\mathrm{~m}, 3 \mathrm{H}), 3.63(\mathrm{~s}, 3 \mathrm{H}), 2.77-2.67(\mathrm{~m}, 1 \mathrm{H}), 2.27-2.14(\mathrm{~m}$, 2H), 2.00-1.86 (m, 2H), $1.28(\mathrm{~d}, J=7.0 \mathrm{~Hz}, 3 \mathrm{H}) ;{ }^{13} \mathrm{C} \mathrm{NMR}\left(125 \mathrm{MHz}, \mathrm{CDCl}_{3}\right): \delta=$ 174.2(s), 146.4(s), 128.6(d), 127.1(d), 126.3(d), 51.6(q), 39.6(d), 33.3(t), 32.4(t), 22.3(q); HRMS (EI) Calcd for $\mathrm{C}_{12} \mathrm{H}_{16} \mathrm{O}_{2}\left[\mathrm{M}^{++}\right]$: 192.11448, found: 192.11518 .

methyl 2-(2,3-dihydro-1H-inden-2-yl)acetate 3as

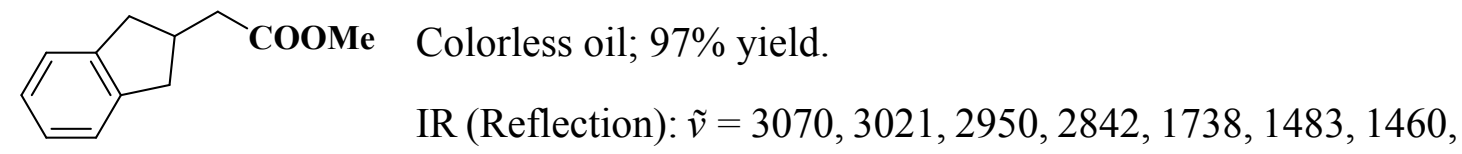
$1436,1371,1335,1297,1270,1224,1199,1150,1051,1025,998,845,779,743 ;{ }^{1} \mathrm{H}$ NMR (500 MHz, $\left.\mathrm{CDCl}_{3}\right): \delta=7.21-7.17(\mathrm{~m}, 2 \mathrm{H}), 7.16-7.11(\mathrm{~m}, 2 \mathrm{H}), 3.70(\mathrm{~s}, 3 \mathrm{H}), 3.15$ (dd, $J=15.5,7.8 \mathrm{~Hz}, 2 \mathrm{H}), 2.94-2.84(\mathrm{~m}, 1 \mathrm{H}), 2.65(\mathrm{dd}, J=15.5,7.3 \mathrm{~Hz}, 2 \mathrm{H}), 2.51(\mathrm{~d}$, $J=7.5 \mathrm{~Hz}, 2 \mathrm{H}) ;{ }^{13} \mathrm{C} \mathrm{NMR}\left(125 \mathrm{MHz}, \mathrm{CDCl}_{3}\right): \delta=173.5(\mathrm{~s}), 142.7(\mathrm{~s}), 126.4(\mathrm{~d}), 124.6(\mathrm{~d})$, 51.7(q), 39.9(t), 39.1(t), 36.2(d); HRMS (EI) Calcd for $\mathrm{C}_{12} \mathrm{H}_{14} \mathrm{O}_{2}\left[\mathrm{M}^{\cdot+}\right]$ : 190.09883, found: 190.09954 .

methyl 2-(2-phenylcyclohexyl)acetate 3at<smiles>COC(=O)CC1CCCCC1c1ccccc1</smiles>

IR (Reflection): $\tilde{v}=3060,3026,2927,2857,1737,1601,1495$, $1449,1435,1379,1351,1325,1283,1221,1165,1116,1019,944,863,756,700,632$; ${ }^{1} \mathrm{H}$ NMR (300 MHz, $\left.\mathrm{CDCl}_{3}\right): \delta=7.26-7.17(\mathrm{~m}, 2 \mathrm{H}), 7.14-7.06(\mathrm{~m}, 3 \mathrm{H}), 3.45(\mathrm{~s}, 3 \mathrm{H})$, 2.92-2.78 (m, 1H), 2.53-2.40 (m, 1H), 2.31-2.18 (m, 1H), 1.94-1.66 (m, 5H), 1.54-1.23 $(\mathrm{m}, 4 \mathrm{H}) ;{ }^{13} \mathrm{C} \mathrm{NMR}\left(125 \mathrm{MHz}, \mathrm{CDCl}_{3}\right): \delta=174.2(\mathrm{~s}), 144.8(\mathrm{~s}), 128.3(\mathrm{~d}), 127.7(\mathrm{~d})$, 126.2(d), 51.4(q), 45.7(d), 37.1(d), 31.7(t), 30.4(t), 26.5(t), 25.3(t), 20.5(t); HRMS (EI) 
Calcd for $\mathrm{C}_{15} \mathrm{H}_{20} \mathrm{O}_{2}\left[\mathrm{M}^{\bullet+}\right]$ : 232.14578, found: 232.14510 .

methyl 2-(1,2,3,4-tetrahydronaphthalen-2-yl)acetate 3au

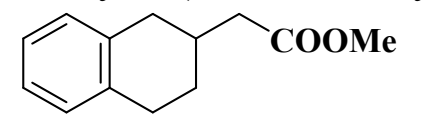

Colorless oil; $85 \%$ yield.

IR (Reflection): $\tilde{v}=3061,3018,2921,2842,1740,1580$, 1495, 1453, 1436, 1358, 1344, 1287, 1252, 1209, 1156, 1104, 1069, 1040, 1012, 948 912, 890, 839, 811, 747; ${ }^{1} \mathrm{H}$ NMR (500 MHz, $\left.\mathrm{CDCl}_{3}\right): \delta=7.14-7.03(\mathrm{~m}, 4 \mathrm{H}), 3.71(\mathrm{~s}$, 3H), $2.91(\mathrm{dd}, J=16.1,4.6 \mathrm{~Hz}, 1 \mathrm{H}), 2.88-2.82(\mathrm{~m}, 2 \mathrm{H}), 2.51(\mathrm{dd}, J=16.3,10.4 \mathrm{~Hz}$, $1 \mathrm{H}), 2.39(\mathrm{~d}, J=7.1 \mathrm{~Hz}, 2 \mathrm{H}), 2.33-2.23(\mathrm{~m}, 1 \mathrm{H}), 2.01-1.93(\mathrm{~m}, 1 \mathrm{H}), 1.54-1.44(\mathrm{~m}, 1 \mathrm{H})$; ${ }^{13} \mathrm{C}$ NMR (125 MHz, $\left.\mathrm{CDCl}_{3}\right): \delta=173.4(\mathrm{~s}), 136.3(\mathrm{~s}), 135.9(\mathrm{~s}), 129.2(\mathrm{~d}), 129.0(\mathrm{~d})$, 125.8(d), 125.7(d), 51.6(q), 41.0(t), 35.8(t), 31.5(d), 29.3(t), 28.9(t); HRMS (EI) Calcd for $\mathrm{C}_{13} \mathrm{H}_{16} \mathrm{O}_{2}\left[\mathrm{M}^{\cdot+}\right]$ : 204.11448, found: 204.11477.

methyl 4-(pyridin-3-yl)butanoate $\mathbf{3 a v}$

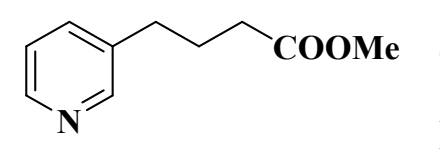

Colorless oil; 75\% yield.

IR (Reflection): $\tilde{v}=3448,3028,2995,2951,2862,1729$, $1591,1574,1478,1435,1422,1367,1312,1249,1172,1142,1106,1044,1025,886$, 863, 826, 792, 713, 628; ${ }^{1} \mathrm{H}$ NMR (500 MHz, $\left.\mathrm{CDCl}_{3}\right): \delta=8.46$ (s, 2H), 7.50 (d, $J=7.7$ $\mathrm{Hz}, 1 \mathrm{H}), 7.25-7.18(\mathrm{~m}, 1 \mathrm{H}), 3.66(\mathrm{~s}, 3 \mathrm{H}), 2.65(\mathrm{t}, J=7.4 \mathrm{~Hz}, 2 \mathrm{H}), 2.34$ (t, $J=7.4 \mathrm{~Hz}$, 2H), 2.00-1.91 (m, 2H); $\left.{ }^{13} \mathrm{C} \mathrm{NMR} \mathrm{(125} \mathrm{MHz,} \mathrm{CDCl}_{3}\right): \delta=173.7(\mathrm{~s}), 150.1(\mathrm{~d}), 147.7(\mathrm{~d})$, 136.0(d), 123.5(d), 51.7(q), 33.3(t), 32.3(t), 26.3(t); HRMS (EI) Calcd for $\mathrm{C}_{10} \mathrm{H}_{13} \mathrm{NO}_{2}$ $\left[\mathrm{M}^{\cdot+}\right]$ : 179.09423, found: 179.09408 .

methyl 4-(isoquinolin-4-yl)butanoate 3aw<smiles>CC(=O)CCCc1cncc2ccccc12</smiles>

Colorless oil; 79\% yield.

IR (Reflection): $\tilde{v}=3854,3750,3595,3447,3003,2950$, $2870,1733,1622,1584,1568,1504,1435,1391,1372$, 1269, 1199, 1169, 1144, 1067, 1020, 895, 862, 788, 751, 691, 656, 623; ${ }^{1} \mathrm{H}$ NMR (500 $\left.\mathrm{MHz}, \mathrm{CDCl}_{3}\right): \delta=9.15(\mathrm{~s}, 1 \mathrm{H}), 8.38(\mathrm{~s}, 1 \mathrm{H}), 8.05(\mathrm{~d}, J=8.5 \mathrm{~Hz}, 1 \mathrm{H}), 7.98(\mathrm{~d}, J=8.1$ 
$\mathrm{Hz}, 1 \mathrm{H}), 7.75$ (t, $J=7.7 \mathrm{~Hz}, 1 \mathrm{H}), 7.61$ (t, $J=7.5 \mathrm{~Hz}, 1 \mathrm{H}), 3.69$ (s, 3H), 3.07 (t, $J=7.6$ $\mathrm{Hz}, 2 \mathrm{H}), 2.44$ (t, $J=7.2 \mathrm{~Hz}, 2 \mathrm{H}), 2.13-2.03(\mathrm{~m}, 2 \mathrm{H}) ;{ }^{13} \mathrm{C} \mathrm{NMR}\left(125 \mathrm{MHz}, \mathrm{CDCl}_{3}\right): \delta=$ 173.9(s), 151.6(d), 142.8(d), 134.8(s), 130.6(d), 128.5(d), 127.1(d), 123.1(d), 51.8(q), 33.62(t), 29.6(t), 25.8(t); HRMS (EI) Calcd for $\mathrm{C}_{14} \mathrm{H}_{15} \mathrm{NO}_{2}\left[\mathrm{M}^{++}\right]: 229.10973$, found: 229.11153.

methyl 4-(pyrimidin-5-yl)butanoate 3ax<smiles>COC(=O)CCCc1cncnc1</smiles>

IR (Reflection): $\tilde{v}=3449,3021,2952,2928,2855,1966$, 1888, 1739, 1583, 1561, 1438, 1411, 1367, 1314, 1254, 1226, 1197, 1169, 1142, 1047, 997, 910, 886, 863, 827, 770, 729, 633; $\left.{ }^{1} \mathrm{H} \mathrm{NMR} \mathrm{(500} \mathrm{MHz,} \mathrm{CDCl}_{3}\right): \delta=9.09(\mathrm{~s}, 1 \mathrm{H})$, $8.60(\mathrm{~s}, 2 \mathrm{H}), 3.69(\mathrm{~s}, 3 \mathrm{H}), 2.67(\mathrm{t}, J=7.7 \mathrm{~Hz}, 2 \mathrm{H}), 2.38$ (t, $J=7.2 \mathrm{~Hz}, 2 \mathrm{H}), 2.02-1.94$ $(\mathrm{m}, 2 \mathrm{H}) ;{ }^{13} \mathrm{C} \mathrm{NMR}\left(125 \mathrm{MHz}, \mathrm{CDCl}_{3}\right): \delta=173.3(\mathrm{~s}), 157.1(\mathrm{~d}), 156.9(\mathrm{~d}), 51.9(\mathrm{q}), 33.2(\mathrm{t})$, 29.8(t), 25.9(t); HRMS (EI) Calcd for $\mathrm{C}_{9} \mathrm{H}_{12} \mathrm{~N}_{2} \mathrm{O}_{2}\left[\mathrm{M}^{\cdot+}\right]$ : 180.08933, found: 180.08795.

methyl 4-(thiophen-3-yl)butanoate 3ay

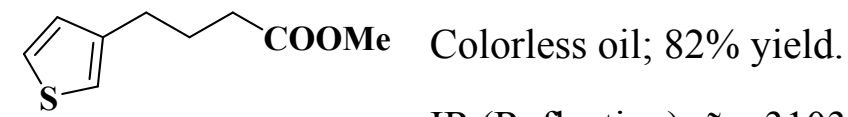

IR (Reflection): $\tilde{v}=3103,2950,2858,1735,1538,1436,1366$, 1337, 1247, 1204, 1174, 1142, 1081, 1052, 999, 940, 850, 833, 771, 688, 634; ${ }^{1} \mathrm{H}$ NMR $\left(300 \mathrm{MHz}, \mathrm{CDCl}_{3}\right): \delta=7.25(\mathrm{dd}, J=4.7,2.9 \mathrm{~Hz}, 1 \mathrm{H}), 6.97-6.92(\mathrm{~m}, 2 \mathrm{H}), 3.67(\mathrm{~s}, 3 \mathrm{H})$, $2.69(\mathrm{t}, J=7.5 \mathrm{~Hz}, 2 \mathrm{H}), 2.35(\mathrm{t}, J=7.5 \mathrm{~Hz}, 2 \mathrm{H}), 2.02-1.92(\mathrm{~m}, 2 \mathrm{H}) ;{ }^{13} \mathrm{C} \mathrm{NMR}(125$ $\left.\mathrm{MHz}, \mathrm{CDCl}_{3}\right): \delta=174.0(\mathrm{~s}), 141.8(\mathrm{~s}), 128.2(\mathrm{~d}), 125.6(\mathrm{~d}), 120.6(\mathrm{~d}), 51.6(\mathrm{q}), 33.5(\mathrm{t})$, 29.6(t), 25.8(t); HRMS (EI) Calcd for $\mathrm{C}_{9} \mathrm{H}_{12} \mathrm{SO}_{2}\left[\mathrm{M}^{\bullet+}\right]$ : 184.05525, found: 184.05439 . 
Methyl 4-(3-bromothiophen-2-yl)butanoate 3az

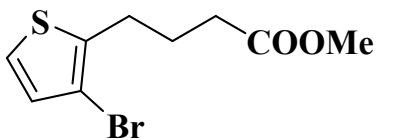

Colorless oil; $77 \%$ yield.

IR (Reflection): $\tilde{v}=3456,3109,3088,2994,2950,2850,1744$ $1521,1437,1368,1346,1317,1251,1229,1198,1171,1120,1083,1043,995,867$, 853, 790, 705, 640; ${ }^{1} \mathrm{H}$ NMR (300 MHz, $\left.\mathrm{CDCl}_{3}\right): \delta=7.13(\mathrm{~d}, J=5.3 \mathrm{~Hz}, 1 \mathrm{H}), 6.91(\mathrm{~d}$, $J=5.3 \mathrm{~Hz}, 1 \mathrm{H}), 3.68(\mathrm{~s}, 3 \mathrm{H}), 2.85(\mathrm{t}, J=7.6 \mathrm{~Hz}, 2 \mathrm{H}), 2.39(\mathrm{t}, J=7.5 \mathrm{~Hz}, 2 \mathrm{H}), 2.04$ 1.95 (m, 2H); ${ }^{13} \mathrm{C} \mathrm{NMR}\left(125 \mathrm{MHz}, \mathrm{CDCl}_{3}\right): \delta=173.7(\mathrm{~s}), 138.4(\mathrm{~s}), 130.1(\mathrm{~d}), 123.5(\mathrm{~d})$, 109.2(s), 51.8(q), 33.2(t), 28.5(t), 25.9(t); HRMS (EI) Calcd for $\mathrm{C}_{9} \mathrm{H}_{11} \mathrm{BrO}_{2} \mathrm{~S}\left[\mathrm{M}^{\bullet+}\right]$ : 261.96631, found: 261.96451.

butyl 4-phenylbutanoate $\mathbf{3 b a}$ Ph COOn-Bu Colorless oil; 88\% yield.

IR (Reflection): $\tilde{v}=3085.42,3063.09,3027.30,2959.35,2934.42,2872.78,1734.90$, $1603.50,1496.50,1454.64,1389.71,1360.36,1306.34,1244.27,1199.41,1175.00$, 1144.94, 1065.49, 1031.15, 964.67, 840.64, 745.93, 700.19; ${ }^{1} \mathrm{H}$ NMR (500 MHz, $\left.\mathrm{CDCl}_{3}\right): \delta=7.28(\mathrm{dd}, J=14.2,6.9 \mathrm{~Hz}, 2 \mathrm{H}), 7.19(\mathrm{t}, J=8.2 \mathrm{~Hz}, 3 \mathrm{H}), 4.08(\mathrm{t}, J=6.7$ $\mathrm{Hz}, 2 \mathrm{H}), 2.66(\mathrm{t}, J=7.6 \mathrm{~Hz}, 2 \mathrm{H}), 2.33$ (t, $J=7.5 \mathrm{~Hz}, 2 \mathrm{H}), 2.01-1.91(\mathrm{~m}, 2 \mathrm{H}), 1.65-1.56$ $(\mathrm{m}, 2 \mathrm{H}), 1.43-1.33(\mathrm{~m}, 2 \mathrm{H}), 0.94(\mathrm{t}, J=7.4 \mathrm{~Hz}, 3 \mathrm{H}) ;{ }^{13} \mathrm{C} \mathrm{NMR}\left(125 \mathrm{MHz}, \mathrm{CDCl}_{3}\right): \delta=$ 173.8(s), 141.6(s), 128.6(d), 128.5(d), 126.1(d), 64.4(t), 35.3(t), 33.8(t), 30.8(t), 26.7(t), 19.3(t), 13.9(q); HRMS (EI) Calcd for $\mathrm{C}_{14} \mathrm{H}_{20} \mathrm{O}_{2}\left[\mathrm{M}^{\bullet+}\right]$ : 220.14578, found: 220.14745 .

\section{1, 4-diphenylbutan-1-one 3ca}

$\int_{\mathbf{O}}^{\mathbf{P h}} \overbrace{}^{\mathbf{P h}}$ Colorless oil; 82\% yield.

IR (Reflection): $\tilde{v}=3337,3103,3061,2943,2861,2658,2342$, $1966,1898,1880,1764,1683,1580,1496,1449,1414,1368,1332,1229,1181,1081$, $1001,972,871,818,793,752,704,6889,620 ;{ }^{1} \mathrm{H} \mathrm{NMR}\left(500 \mathrm{MHz}, \mathrm{CDCl}_{3}\right): \delta=7.93$ $(\mathrm{d}, J=8.6 \mathrm{~Hz}, 2 \mathrm{H}), 7.55(\mathrm{t}, J=7.4 \mathrm{~Hz}, 1 \mathrm{H}), 7.45(\mathrm{t}, J=7.7 \mathrm{~Hz}, 2 \mathrm{H}), 7.30(\mathrm{t}, J=7.5$ $\mathrm{Hz}, 2 \mathrm{H}), 7.24-7.18(\mathrm{~m}, 3 \mathrm{H}), 2.99$ (t, $J=7.3 \mathrm{~Hz}, 2 \mathrm{H}), 2.73$ (t, $J=7.6 \mathrm{~Hz}, 2 \mathrm{H}), 2.13-2.05$ 
(m, 2H); ${ }^{13} \mathrm{C}$ NMR (125 MHz, $\left.\mathrm{CDCl}_{3}\right): \delta=200.3(\mathrm{~s}), 141.8(\mathrm{~s}), 137.2(\mathrm{~s}), 133.1(\mathrm{~d})$, 128.7(d), 128.7(d), 128.5(d), 128.2(d), 126.1(d), 37.8(t), 35.3(t), 25.8(t); HRMS (EI) Calcd for $\mathrm{C}_{16} \mathrm{H}_{16} \mathrm{O}\left[\mathrm{M}^{\bullet+}\right.$ ]: 224.11957, found: 224.11876 .

2-methyl-1, 4-diphenylbutan-1-one 3da

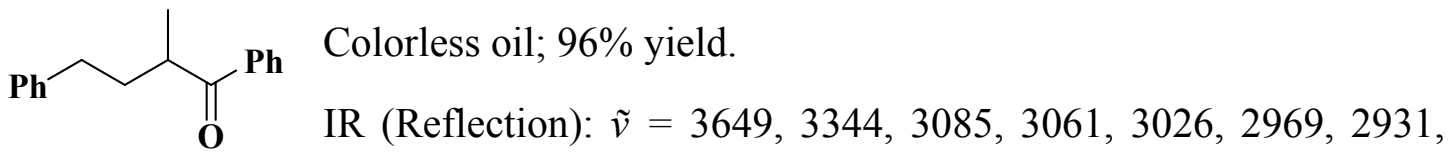
2859, 1962, 1814, 1678, 1596, 1579, 1495, 1449, 1375, 1278, 1225, 1177, 1126, 1073, 1047, 1030, 1001, 972, 930, 914, 846, 824, 794, 747, 696, 654; ${ }^{1} \mathrm{H}$ NMR (300 MHz, $\left.\mathrm{CDCl}_{3}\right): \delta=7.92-7.83(\mathrm{~m}, 2 \mathrm{H}), 7.60-7.51(\mathrm{~m}, 1 \mathrm{H}), 7.49-7.40(\mathrm{~m}, 2 \mathrm{H}), 7.33-7.24(\mathrm{~m}$, 2H), 7.24-7.12 (m, 3H), 3.54-3.43 (m, 1H), 2.66 (t, $J=7.8 \mathrm{~Hz}, 2 \mathrm{H}), 2.25-2.13(\mathrm{~m}, 1 \mathrm{H})$, 1.83-1.71 (m, 1H), $\left.1.25(\mathrm{~d}, J=6.9 \mathrm{~Hz}, 3 \mathrm{H}) ;{ }^{13} \mathrm{C} \mathrm{NMR} \mathrm{(125} \mathrm{MHz,} \mathrm{CDCl}_{3}\right): \delta=204.2(\mathrm{~s})$, 141.9(s), 136.7(s), 133.0(d), 128.7(d), 128.6(d), 128.5(d), 128.4(d), 126.1(d), 39.9(d), 35.3(t), 33.6(t), 17.4(q); HRMS (EI) Calcd for $\mathrm{C}_{17} \mathrm{H}_{18} \mathrm{O}\left[\mathrm{M}^{\cdot+}\right]$ : 238.13522, found: 238.13658 .

2, 2-dimethyl-1, 4-diphenylbutan-1-one 3ea

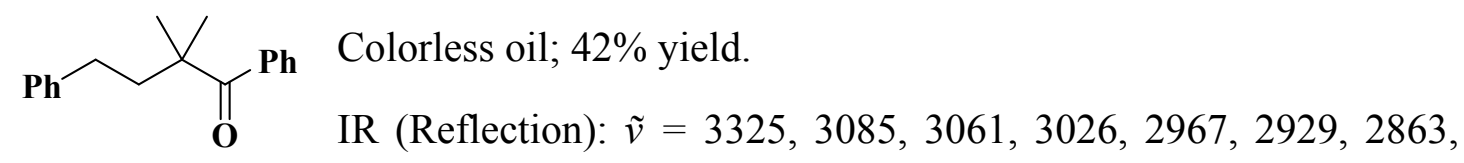
1954, 1869, 1809, 1673, 1598, 1582, 1497, 1470, 1455, 1387, 1366, 1311, 1257, 1197 , $1179,1074,1031,1002,986,960,850,794,749,718,700,636,621 ;{ }^{1} \mathrm{H}$ NMR $(500$ $\left.\mathrm{MHz}, \mathrm{CDCl}_{3}\right): \delta=7.74-7.70(\mathrm{~m}, 2 \mathrm{H}), 7.51-7.46(\mathrm{~m}, 1 \mathrm{H}), 7.45-7.39(\mathrm{~m}, 2 \mathrm{H}), 7.24(\mathrm{t}, J$ $=7.5 \mathrm{~Hz}, 2 \mathrm{H}), 7.16(\mathrm{t}, J=7.4 \mathrm{~Hz}, 1 \mathrm{H}), 7.09-7.03(\mathrm{~m}, 2 \mathrm{H}), 2.56-2.49(\mathrm{~m}, 2 \mathrm{H}), 2.09-2.03$ (m, 2H), 1.40 (s, 6H); $\left.{ }^{13} \mathrm{C} \mathrm{NMR} \mathrm{(125} \mathrm{MHz,} \mathrm{CDCl}_{3}\right): \delta=208.9(\mathrm{~s}), 142.4(\mathrm{~s}), 139.2(\mathrm{~s})$, 131.1(d), 128.5(d), 128.4(d), 128.3(d), 127.8(d), 126.0(d), 125.8(d), 48.1(s), 43.4(t), 31.5(t), 26.4(q); HRMS (EI) Calcd for $\mathrm{C}_{18} \mathrm{H}_{20} \mathrm{O}\left[\mathrm{M}^{\cdot+}\right]$ : 252.15087, found: 252.15759.

2-phenethylcyclopentan-1-one $\mathbf{3 f a}$<smiles>O=C1CCCC1CCc1ccccc1</smiles> 
IR (Reflection): $\tilde{v}=3451,3084,3061,3026,2958,2937,2860,1948,1872,1742,1603$, 1584, 1496, 1454, 1405, 1353, 1326, 1272, 1155, 1116, 1072, 1030, 1004, 930, 917 , 822, 749, 699; ${ }^{1} \mathrm{H}$ NMR (500 MHz, $\left.\mathrm{CDCl}_{3}\right): \delta=7.23-7.18$ (m, 2H), 7.15-7.08 (m, 3H), 2.70-2.62 (m, 1H), 2.62-2.54 (m, 1H), 2.27-2.13 (m, 2H), 2.10-2.00 (m, 2H), 2.00-1.89 $(\mathrm{m}, 2 \mathrm{H}), 1.75-1.64(\mathrm{~m}, 1 \mathrm{H}), 1.53-1.46(\mathrm{~m}, 2 \mathrm{H}) ;{ }^{13} \mathrm{C}$ NMR $\left(125 \mathrm{MHz}, \mathrm{CDCl}_{3}\right): \delta=$ 221.4(s), 141.8(s), 128.6(d), 128.5(d), 126.1(d), 48.5(t), 38.3(d), 33.8(t), 31.5(t), 29.9(t), 20.9(t); HRMS (EI) Calcd for $\mathrm{C}_{13} \mathrm{H}_{16} \mathrm{O}\left[\mathrm{M}^{\cdot+}\right]$ : 188.11957, found: 188.11853 .

(4, 4, 4-trifluorobutyl)benzene 3ga

$\mathrm{CF}_{3}$ Colorless oil; $62 \%$ yield.

IR (Reflection): $\tilde{v}=2924,2853,1734,1599,1463,1380,1256,1206,1134,1008,749$, 699; ${ }^{1} \mathrm{H} \mathrm{NMR}\left(500 \mathrm{MHz}, \mathrm{CDCl}_{3}\right): \delta=7.30(\mathrm{t}, J=7.4 \mathrm{~Hz}, 2 \mathrm{H}), 7.22(\mathrm{t}, J=7.4 \mathrm{~Hz}, 1 \mathrm{H})$, $7.18(\mathrm{~d}, J=7.0 \mathrm{~Hz}, 2 \mathrm{H}), 2.70(\mathrm{t}, J=7.6 \mathrm{~Hz}, 2 \mathrm{H}), 2.14-2.02(\mathrm{~m}, 2 \mathrm{H}), 1.94-1.85$ (m, $2 \mathrm{H}) ;{ }^{13} \mathrm{C} \mathrm{NMR}\left(125 \mathrm{MHz}, \mathrm{CDCl}_{3}\right): \delta=140.8(\mathrm{~s}), 128.7(\mathrm{~d}), 128.5(\mathrm{~d}), 126.4(\mathrm{~d}), 34.8(\mathrm{t})$, $33.2(\mathrm{t}, \mathrm{q}, J=28.2 \mathrm{~Hz}), 23.6(\mathrm{t}, \mathrm{q}, J=2.8 \mathrm{~Hz}) ;{ }^{19} \mathrm{~F} \mathrm{NMR}\left(283 \mathrm{MHz}, \mathrm{CDCl}_{3}\right): \delta=-66.18$; HRMS (EI) Calcd for $\mathrm{C}_{10} \mathrm{H}_{11} \mathrm{~F}_{3}\left[\mathrm{M}^{\bullet+}\right]$ : 188.08074, found: 188.08109 .

\section{4-phenylbutanenitrile $\mathbf{3 h a}$}

Ph CN Colorless oil; 88\% yield.

IR (Reflection): $\tilde{v}=3085,3063,3028,2927,2857,2246,1953,1879,1811,1733,1603$, 1584, 1496, 1455, 1425, 1350, 1326, 1179, 1082, 1030, 1003, 911, 844, 801, 747, 700; ${ }^{1} \mathrm{H}$ NMR (500 MHz, $\left.\mathrm{CDCl}_{3}\right): \delta=7.32(\mathrm{dd}, J=10.2,4.6 \mathrm{~Hz}, 2 \mathrm{H}), 7.23(\mathrm{dd}, J=8.4,6.4$ $\mathrm{Hz}, 1 \mathrm{H}), 7.21-7.17(\mathrm{~m}, 2 \mathrm{H}), 2.78(\mathrm{t}, J=7.4 \mathrm{~Hz}, 2 \mathrm{H}), 2.32$ (t, $J=7.1 \mathrm{~Hz}, 2 \mathrm{H}), 2.02-1.96$ $(\mathrm{m}, 2 \mathrm{H}) ;{ }^{13} \mathrm{C} \mathrm{NMR}\left(125 \mathrm{MHz}, \mathrm{CDCl}_{3}\right): \delta=139.9(\mathrm{~s}), 128.8(\mathrm{~d}), 128.6(\mathrm{~d}), 126.7(\mathrm{~d})$, 119.6(s), 34.5(t), 27.1(t), 16.5(t); HRMS (EI) Calcd for $\mathrm{C}_{10} \mathrm{H}_{11} \mathrm{~N}\left[\mathrm{M}^{\cdot+}\right]$ : 145.08860, found: 145.08793 .

1-(3-phenylpropyl)-2,4-bis(trifluoromethyl)benzene 3ia

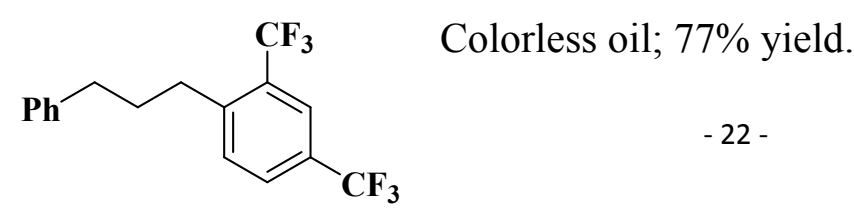


IR (Reflection): $\tilde{v}=3087,3065,3029,2937,2864,1943,1811,1628,1604,1586,1497$, 1455, 1347, 1302, 1276, 1205, 1176, 1127, 1085, 1057, 1030, 912, 841, 747, 699, 673, 642; ${ }^{1} \mathrm{H}$ NMR $\left(500 \mathrm{MHz}, \mathrm{CDCl}_{3}\right): \delta=7.88(\mathrm{~s}, 1 \mathrm{H}), 7.71(\mathrm{~d}, J=8.0 \mathrm{~Hz}, 1 \mathrm{H}), 7.45(\mathrm{~d}, J$ $=8.1 \mathrm{~Hz}, 1 \mathrm{H}), 7.31(\mathrm{t}, J=7.6 \mathrm{~Hz}, 2 \mathrm{H}), 7.22(\mathrm{t}, J=7.2 \mathrm{~Hz}, 3 \mathrm{H}), 2.89(\mathrm{t}, J=7.8 \mathrm{~Hz}, 2 \mathrm{H})$, $2.74(\mathrm{t}, J=7.7 \mathrm{~Hz}, 2 \mathrm{H}), 2.03-1.93(\mathrm{~m}, 2 \mathrm{H}) ;{ }^{13} \mathrm{C} \mathrm{NMR}\left(125 \mathrm{MHz}, \mathrm{CDCl}_{3}\right): \delta=141.5(\mathrm{~s})$, 131.6(d), 128.5(d), 128.4(d), 126.1(d), 35.8(t), 33.1(t), 32.2(t); ${ }^{19}$ F NMR (283 MHz, $\mathrm{CDCl}_{3}$ ): $\delta=-60.20,-62.72$; HRMS (EI) Calcd for $\mathrm{C}_{17} \mathrm{H}_{14} \mathrm{~F}_{6}\left[\mathrm{M}^{\cdot+}\right]$ : 332.09942, found: 332.09844.

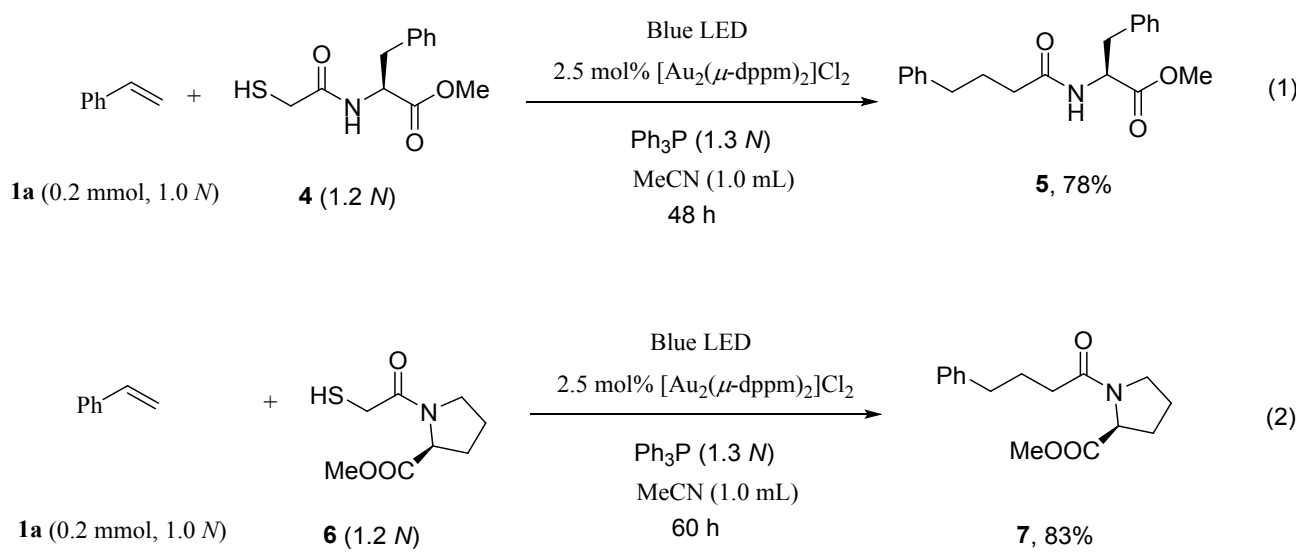

Scheme S1. Modification of small amino acid fragments.

In a $10 \mathrm{~mL}$ dried pyrex screw-top reaction tube, $\left[\mathrm{Au}_{2}(\mu \text {-dppm })_{2}\right] \mathrm{Cl}_{2}(2.5 \mathrm{~mol} \%), \mathrm{Ph}_{3} \mathrm{P}$ (1.3 eqs) was dissolved in MeCN (1.0 mL). Then the alkenes 1a $(0.2 \mathrm{mmol}, 1.0 \mathrm{eq})$ and the mercaptans (1.2 eqs) were added. The reaction mixture was then cooled to $-78{ }^{\circ} \mathrm{C}$ and degassed via vacuum evacuation ( $5 \mathrm{~min}$ ), backfilled with nitrogen, and warmed to room temperature. This process was repeated three times. After the reaction was thoroughly degassed, irradiation of the reaction tubes was conducted with blue leds and the white solid byproduct $\mathrm{Ph}_{3}$ PS precipitated. When the reaction finished, the reaction mixture was diluted with DCM. After removal of the solvent in vacuo, the resulting crude products were subjected to flash column chromatography on silica gel to give 
desired products. The products were characterized with IR, ${ }^{1} \mathrm{H}$ NMR, ${ }^{13} \mathrm{C}$ NMR and HRMS.

methyl (4-phenylbutanoyl)-L-phenylalaninate 5<smiles>COC(=O)C(Cc1ccccc1)NC(=O)CCCc1ccccc1</smiles>
$78 \%$ yield.

IR (Reflection): $\tilde{v}=3292,3062,3027,2951,2928,2857$, $1951,1878,1745,1650,1541,1497,1454,1370,1278$, $1248,1214,1177,1080,1030,746,700 ;{ }^{1} \mathrm{H} \mathrm{NMR}\left(500 \mathrm{MHz}, \mathrm{CDCl}_{3}\right) \delta=7.31-7.26$ (m, 3H), 7.26-7.22 (m, 2H), 7.19 (t, $J=7.4 \mathrm{~Hz}, 1 \mathrm{H}), 7.14$ (d, $J=7.3 \mathrm{~Hz}, 2 \mathrm{H}), 7.08$ (d, $J=6.8 \mathrm{~Hz}, 2 \mathrm{H}), 5.83(\mathrm{~d}, J=7.4 \mathrm{~Hz}, 1 \mathrm{H}), 4.91(\mathrm{dd}, J=12.7,6.7 \mathrm{~Hz}, 1 \mathrm{H}), 3.73(\mathrm{~s}, 3 \mathrm{H})$, $3.16(\mathrm{dd}, J=13.9,5.8 \mathrm{~Hz}, 1 \mathrm{H}), 3.09$ (dd, $J=13.9,5.8 \mathrm{~Hz}, 1 \mathrm{H}), 2.61$ (t, $J=7.5 \mathrm{~Hz}, 2 \mathrm{H})$, $2.18(\mathrm{t}, J=7.6 \mathrm{~Hz}, 2 \mathrm{H}), 1.98-1.89(\mathrm{~m}, 2 \mathrm{H}) ;{ }^{13} \mathrm{C} \mathrm{NMR}\left(125 \mathrm{MHz}, \mathrm{CDCl}_{3}\right) \delta=172.4(\mathrm{~s})$, 172.29(s), 141.5(d), 136.0(d), 129.4(d), 128.7(d), 128.6(d), 128.5(d), 127.3(d), 126.1(d), 53.0(d), 52.5(d), 38.0(t), 35.7(t), 35.2(t), 27.0(t); HRMS (DART) Calcd for $\mathrm{C}_{20} \mathrm{H}_{24} \mathrm{NO}_{3}$ $[\mathrm{M}+1]$ : 326.1751, found: 326.1758 .

methyl (4-phenylbutanoyl)-L-prolinate 7<smiles>COC(=O)[C@H]1CCCN1C(=O)CCCc1ccccc1</smiles>

$83 \%$ yield.

IR (Reflection): $\tilde{v}=3478,3060,3025,2951,2876,1743,1635$, $1496,1418,1363,1322,1280,1172,1096,1029,911,874,749$, 701; ${ }^{1} \mathrm{H}$ NMR $\left(500 \mathrm{MHz}, \mathrm{CDCl}_{3}\right) \delta=7.29-7.26(\mathrm{~m}, 2 \mathrm{H}), 7.21-7.15(\mathrm{~m}, 3 \mathrm{H}), 4.48(\mathrm{dd}$, $J=8.4,3.8 \mathrm{~Hz}, 1 \mathrm{H}), 3.73(\mathrm{~s}, 3 \mathrm{H}), 3.59-3.52(\mathrm{~m}, 1 \mathrm{H}), 3.44-3.37(\mathrm{~m}, 1 \mathrm{H}), 2.68(\mathrm{t}, J=7.5$ $\mathrm{Hz}, 2 \mathrm{H}), 2.38-2.22(\mathrm{~m}, 2 \mathrm{H}), 2.22-2.05(\mathrm{~m}, 2 \mathrm{H}), 2.05-1.96(\mathrm{~m}, 4 \mathrm{H}) ;{ }^{13} \mathrm{C}$ NMR (125 MHz, $\left.\mathrm{CDCl}_{3}\right) \delta=173.1(\mathrm{~s}), 171.8(\mathrm{~s}), 141.9(\mathrm{~s}), 128.7(\mathrm{~s}), 128.4(\mathrm{~s}), 126.0(\mathrm{~s})$, 58.7(d), 52.3(d), 47.0(t), 35.2(t), 33.5(t), 29.3(t), 26.1(t), 24.9(t); HRMS (EI) Calcd for $\mathrm{C}_{16} \mathrm{H}_{21} \mathrm{NO}_{3}\left[\mathrm{M}^{\cdot+}\right.$ ]: 275.15159 , found: 275.15027 . 
1)

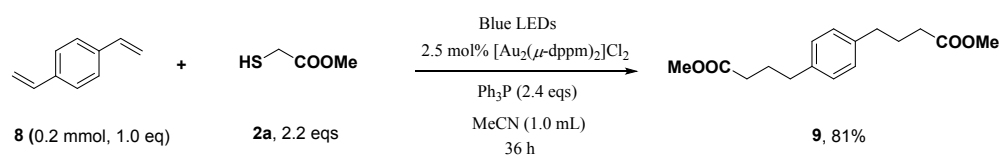

2)

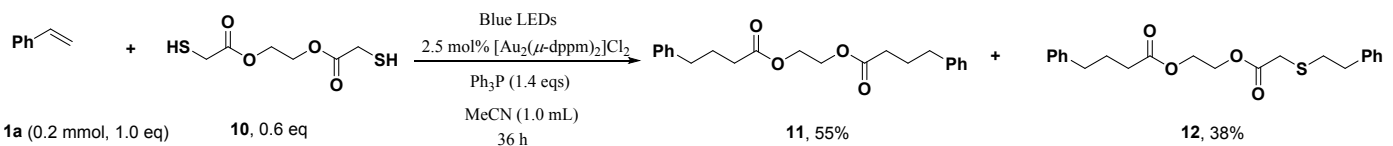

3)

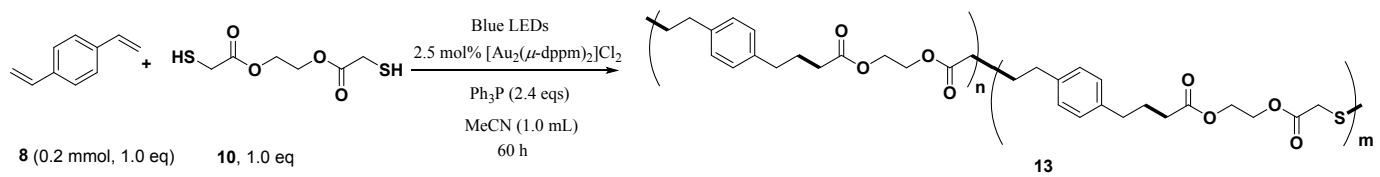

$8(0.2 \mathrm{mmol}, 1.0 \mathrm{eq}) \quad \mathbf{1 0}, 1.0 \mathrm{eq}$ $60 \mathrm{~h}$

Scheme S2. Application for polymer synthesis.

In a $10 \mathrm{~mL}$ dried pyrex screw-top reaction tube, $\left[\mathrm{Au}_{2}(\mu \text {-dppm })_{2}\right] \mathrm{Cl}_{2}(2.5 \mathrm{~mol} \%), \mathrm{Ph}_{3} \mathrm{P}$ (2.4 eqs) was dissolved in $\mathrm{MeCN}(1.0 \mathrm{~mL})$. Then the alkenes $8(0.2 \mathrm{mmol}, 1.0 \mathrm{eq})$ and the mercaptans 10 (1.0 eq) were added. The reaction mixture was then cooled to $-78^{\circ} \mathrm{C}$ and degassed via vacuum evacuation ( $5 \mathrm{~min}$ ), backfilled with nitrogen, and warmed to room temperature. This process was repeated three times. After the reaction was thoroughly degassed, irradiation of the reaction tubes was conducted with blue leds and the white solid byproduct $\mathrm{Ph}_{3} \mathrm{PS}$ precipitated. When the reaction finished, the reaction mixture was dissolved in DCM, followed by removing most of the solvent in vacuo, the resulting solution was added dropwise into much amount of cold methanol (300 $\mathrm{mL}$ ) to get the desired polymer. Products $\mathbf{9 , 1 1}$ and $\mathbf{1 2}$ are purified by flash column chromatography on silica gel. 
dimethyl 4,4'-(1,4-phenylene)dibutyrate 9<smiles>COC(=O)CCCc1ccc(CCCC(=O)OC)cc1</smiles>

IR (Reflection): $\tilde{\mathrm{v}}=2950,2860,1736,1514$, 1437, 1366, 1326, 1300, 1247, 1200, 1172, 1147, 999, 887, 838, 806; ${ }^{1} \mathrm{H}$ NMR $(300$ $\left.\mathrm{MHz}, \mathrm{CDCl}_{3}\right): \delta=7.09(\mathrm{~s}, 4 \mathrm{H}), 3.66(\mathrm{~s}, 6 \mathrm{H}), 2.61(\mathrm{t}, J=7.6 \mathrm{~Hz}, 4 \mathrm{H}), 2.33(\mathrm{t}, J=7.5$ $\mathrm{Hz}, 4 \mathrm{H}), 2.00-1.87(\mathrm{~m}, 4 \mathrm{H}) ;{ }^{13} \mathrm{C} \mathrm{NMR}\left(75 \mathrm{MHz}, \mathrm{CDCl}_{3}\right): \delta=174.1(\mathrm{~s}), 139.1(\mathrm{~s})$, 128.6(d), 51.6(q), 34.9(t), 33.6(t), 26.7(t). HRMS (ESI) Calcd for $\mathrm{C}_{16} \mathrm{H}_{23} \mathrm{O}_{4}[\mathrm{M}+\mathrm{H}]$ : 279.1591, found: 279.1595 .

ethane-1,2-diyl bis(4-phenylbutanoate) $\mathbf{1 1}$<smiles>O=C(CCCc1ccccc1)OCCOC(=O)CCCc1ccccc1</smiles>

$55 \%$ yield.

IR (Reflection): $\tilde{v}=3061,3026,2925,2855$ $1736,1603,1497,1454,1379,1139,1082$, 959, 879, 746, 700; ${ }^{1} \mathrm{H}$ NMR (400 MHz, $\left.\mathrm{CDCl}_{3}\right): \delta=7.31-7.26(\mathrm{~m}, 4 \mathrm{H}), 7.22-7.14(\mathrm{~m}$, 6H), 4.27 (s, 4H), 2.65 (t, $J=7.6 \mathrm{~Hz}, 4 \mathrm{H}), 2.35$ (t, $J=7.5 \mathrm{~Hz}, 4 \mathrm{H}), 2.01-1.90(\mathrm{~m}, 4 \mathrm{H})$. ${ }^{13} \mathrm{C}$ NMR (101 MHz, $\left.\mathrm{CDCl}_{3}\right): \delta=173.4(\mathrm{~s}), 141.4(\mathrm{~s}), 128.6(\mathrm{~d}), 128.6(\mathrm{~d}), 126.2(\mathrm{~d})$, 62.2(t), 35.2(t), 33.6(t), 26.6(t). HRMS (ESI) Calcd for $\mathrm{C}_{22} \mathrm{H}_{26} \mathrm{NaO}_{4}[\mathrm{M}+\mathrm{Na}]$ : 377.1723 , found: 377.1724 .

2-(2-(phenethylthio)acetoxy)ethyl 4-phenylbutanoate 12<smiles>O=C(CCCc1ccccc1)OCCOC(=O)CSCCI</smiles>
$38 \%$ yield.

IR (Reflection): $\tilde{v}=3085,3062,3026,2925$ $2854,1735,1603,1497,1454,1414,1377$, $1276,1127,1050,1031,963,747,700 ;{ }^{1} \mathrm{H}$ NMR $\left(400 \mathrm{MHz}, \mathrm{CDCl}_{3}\right): \delta=7.32-7.15(\mathrm{~m}$, $10 \mathrm{H}), 4.36-4.32(\mathrm{~m}, 2 \mathrm{H}), 4.32-4.27(\mathrm{~m}, 2 \mathrm{H}), 3.24(\mathrm{~s}, 2 \mathrm{H}), 2.90(\mathrm{~s}, 4 \mathrm{H}), 2.64(\mathrm{t}, J=7.6$ $\mathrm{Hz}, 2 \mathrm{H}), 2.32(\mathrm{t}, J=7.5 \mathrm{~Hz}, 2 \mathrm{H}), 1.99-1.90(\mathrm{~m}, 2 \mathrm{H}) .{ }^{13} \mathrm{C} \mathrm{NMR}\left(101 \mathrm{MHz}, \mathrm{CDCl}_{3}\right): \delta=$ 173.3(s), 170.4(s), 141.4(s), 140.2(s), 128.7(d), 128.7(d), 128.6(d), 128.6(d), 126.6(d), 126.2(d), 63.2(t), 62.0(t), 35.8(t), 35.2(t), 34.2(t), 33.6(t), 33.5(t), 26.5(t). HRMS (ESI) 
Calcd for $\mathrm{C}_{22} \mathrm{H}_{26} \mathrm{NaO}_{4} \mathrm{~S}$ [M+Na]: 409.1444, found: 409.1446 .

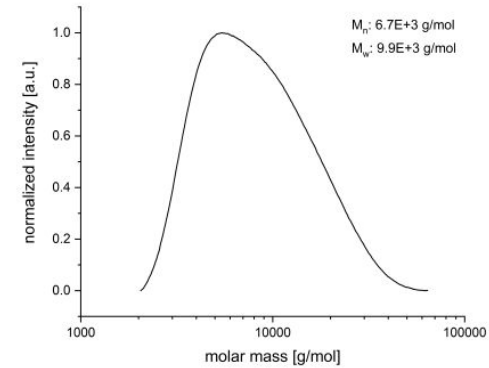

Figure S1. GPC data of polymer 13
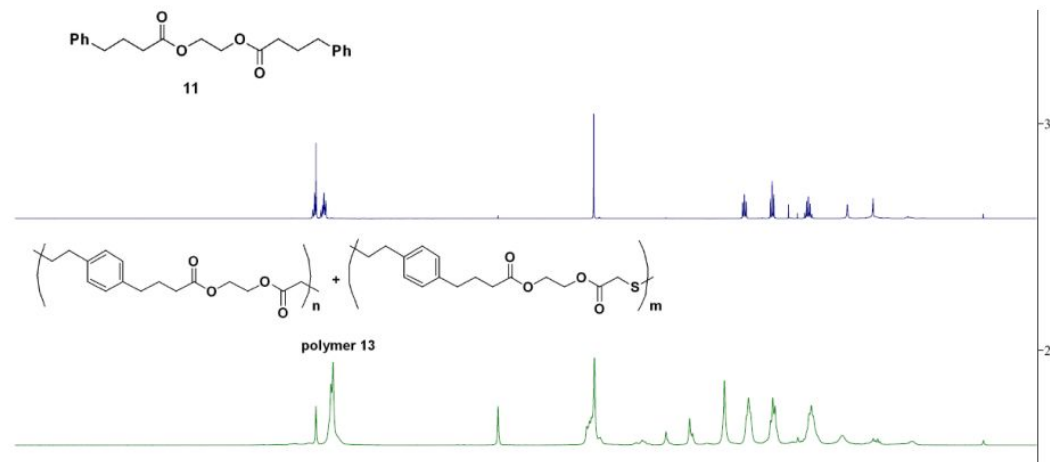

$\mathrm{Ph}_{12}^{\mathrm{O}} \sim_{\mathrm{O}}^{\mathrm{O}} \sim^{\mathrm{Ph}}$

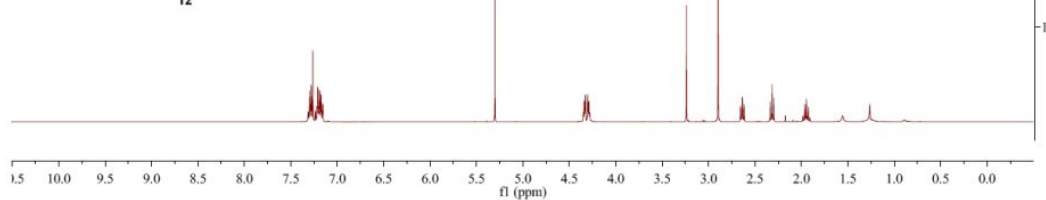

Figure S2. ${ }^{1} \mathrm{H}$ NMR spectrum identification of polymer $\mathbf{1 3}$ 


\section{Mechanism study}

a) Inhibition experiment

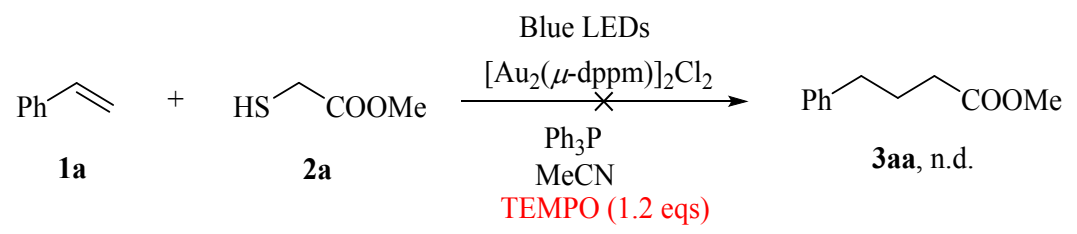

In the model reaction of $\mathbf{1 a}$ and $\mathbf{2 a}$ in the presence of $\left[\mathrm{Au} 2(\mu-\mathrm{dppm})_{2}\right] \mathrm{Cl}_{2}(2.5 \mathrm{~mol} \%)$, 1.2 equiv TEMPO (tetramethylpiperdinyloxy free radical, a classical radical trapping reagent) was added. The reaction mixture was irradiated under blue leds for 24 hours. From the result of crude ${ }^{1} \mathrm{H}-\mathrm{NMR}$ and GC-MS spectrum, we found that no desired product 3aa was formed.

b) Radical clock reaction

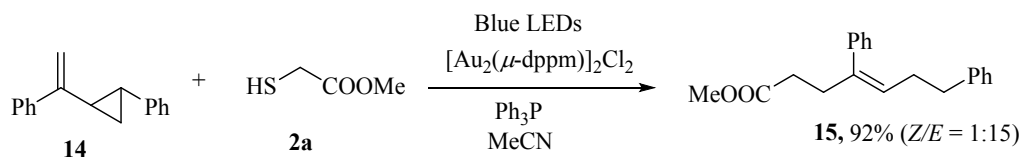

In a $10 \mathrm{~mL}$ dried pyrex screw-top reaction tube, $\left[\mathrm{Au}_{2}(\mu \text {-dppm })_{2}\right] \mathrm{Cl}_{2}(2.5 \mathrm{~mol} \%), \mathrm{Ph}_{3} \mathrm{P}$ (1.3 eqs)was dissolved in $\mathrm{MeCN}(1.0 \mathrm{~mL})$. Then the alkenes $14(0.2 \mathrm{mmol}, 1 \mathrm{~N})$ and the mercaptans 15 (1.2 eq) were added. The reaction mixture was then cooled to $-78{ }^{\circ} \mathrm{C}$ and degassed via vacuum evacuation ( $5 \mathrm{~min}$ ), backfilled with nitrogen, and warmed to room temperature. This process was repeated three times. After the reaction was thoroughly degassed, irradiation of the reaction tubes was conducted with blue leds for $24 \mathrm{~h}$ and the white solid byproduct $\mathrm{Ph}_{3} \mathrm{PS}$ is precipitated. When the reaction finished, the reaction mixture was diluted with DCM. After removal of the solvent in vacuum, the resulting crude products were subjected to flash column chromatography on silica gel to give desired products $\mathbf{1 5}$ in $92 \%$ yield. 
methyl (Z)-4,7-diphenylhept-4-enoate 15<smiles>CC(=O)CC/C(=C\CCc1ccccc1)c1ccccc1</smiles>

Colorless oil; $92 \%$ yield.

IR (Reflection): $\tilde{v}=3456,3082 ., 3060,3026,2949,2925$, $2856,1948,1878,1741,1601,1574,1495,1454,1436,1356,1278,1254,1196,1164$, $1076,1051,1029,986,889,866,764,748,700 ;{ }^{1} \mathrm{H} \mathrm{NMR}\left(500 \mathrm{MHz}, \mathrm{CDCl}_{3}\right): \delta=7.33-$ $7.28(\mathrm{~m}, 6 \mathrm{H}), 7.24-7.21(\mathrm{~m}, 4 \mathrm{H}), 5.73(\mathrm{t}, J=7.3 \mathrm{~Hz}, 1 \mathrm{H}), 3.61(\mathrm{~s}, 3 \mathrm{H}), 2.77(\mathrm{t}, J=7.3$ $\mathrm{Hz}, 4 \mathrm{H}), 2.55$ (q, $J=7.5 \mathrm{~Hz}, 2 \mathrm{H}), 2.19(\mathrm{t}, J=7.4 \mathrm{~Hz}, 2 \mathrm{H}) ;{ }^{13} \mathrm{C}$ NMR $\left(125 \mathrm{MHz}, \mathrm{CDCl}_{3}\right)$ : $\delta=173.7(\mathrm{~s}), 142.3(\mathrm{~s}), 141.9(\mathrm{~s}), 139.1(\mathrm{~s}), 129.2(\mathrm{~d}), 128.7(\mathrm{~d}), 128.5(\mathrm{~d}), 128.5(\mathrm{~d})$, 127.0(d), 126.6(d), 126.1(d), 51.6(q), 36.1(t), 33.1(t), 30.6(t), 25.3(t); HRMS (EI) Calcd for $\mathrm{C}_{20} \mathrm{H}_{22} \mathrm{O}_{2}\left[\mathrm{M}^{\cdot+}\right]$ : 294.16143, found: 294.16146.

\section{c) Deuterium experiment}

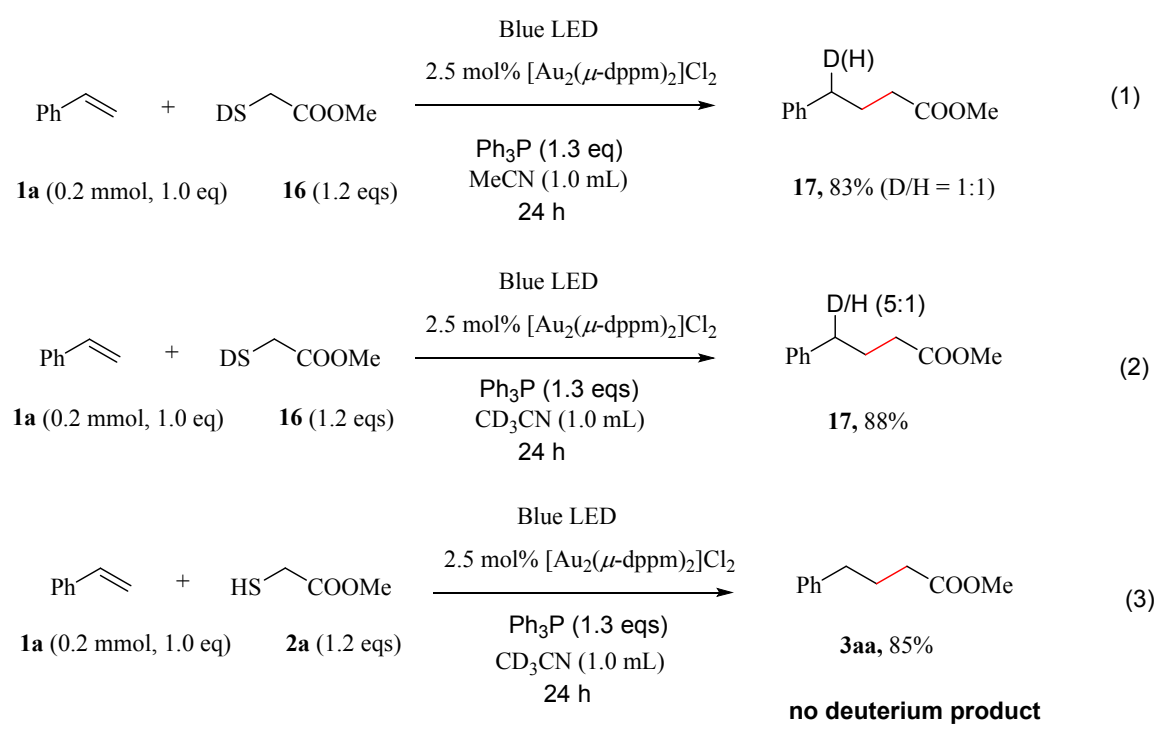

In a $10 \mathrm{~mL}$ dried pyrex screw-top reaction tube, $\left[\mathrm{Au}_{2}(\mu \text {-dppm })_{2}\right] \mathrm{Cl}_{2}(2.5 \mathrm{~mol} \%), \mathrm{Ph}_{3} \mathrm{P}$ (1.3 eqs)was dissolved in $\mathrm{MeCN}(1.0 \mathrm{~mL})$. Then the alkenes $\mathbf{1 a}(0.2 \mathrm{mmol}, 1.0 \mathrm{eq})$ and the mercaptans (1.2 eqs) were added. The reaction mixture was then cooled to $-78{ }^{\circ} \mathrm{C}$ and degassed via vacuum evacuation ( $5 \mathrm{~min}$ ), backfilled with nitrogen, and warmed to room temperature. This process was repeated three times. After the reaction was thoroughly degassed, irradiation of the reaction tubes was conducted with blue leds for $24 \mathrm{~h}$ and the white solid byproduct $\mathrm{Ph}_{3} \mathrm{PS}$ precipitated. When the reaction finished, the 
reaction mixture was extracted with ethyl acetate. The combined organic phase were dried over MgSO4 and filtered. After removal of the solvent in vacuum, the resulting crude products were subjected to flash column chromatography on silica gel to give desired products.

methyl 4-phenylbutanoate $\mathbf{1 6}$ (1:1 D/H mixtures)

Colorless oil; 83\% yield.
COOMe 1739, 1603, 1496, 1453, 1436, 1366, 1310, 1250, 1203, 1164, 1081, 1030, 854, 745, 700.48; ${ }^{1} \mathrm{H} \mathrm{NMR}\left(500 \mathrm{MHz}, \mathrm{CDCl}_{3}\right): \delta=7.29$ (t, $\left.J=7.5 \mathrm{~Hz}, 2 \mathrm{H}\right), 7.19$ (t, $J=8.6 \mathrm{~Hz}$, $3 \mathrm{H}), 3.67(\mathrm{~s}, 3 \mathrm{H}), 2.66(\mathrm{t}, J=7.6 \mathrm{~Hz}, 1.5 \mathrm{H}), 2.34(\mathrm{t}, J=7.5 \mathrm{~Hz}, 2 \mathrm{H}), 1.99-1.93(\mathrm{~m}, 2 \mathrm{H})$; ${ }^{13} \mathrm{C}$ NMR (125 MHz, $\mathrm{CDCl}_{3}$ ): $\delta=174.1(\mathrm{~s}), 141.5(\mathrm{~s}), 128.6(\mathrm{~d}), 128.5(\mathrm{~d}), 126.1(\mathrm{~d})$, 51.7(q), 35.3(t), 33.5(t), 33.5(t) 26.6(t), 26.5(t); HRMS (EI) Calcd for $\mathrm{C}_{11} \mathrm{H}_{13} \mathrm{O}_{2} \mathrm{D}\left[\mathrm{M}^{\bullet+}\right]$ : 179.10511, found: 179.10339 .

\section{d) Control experiments}

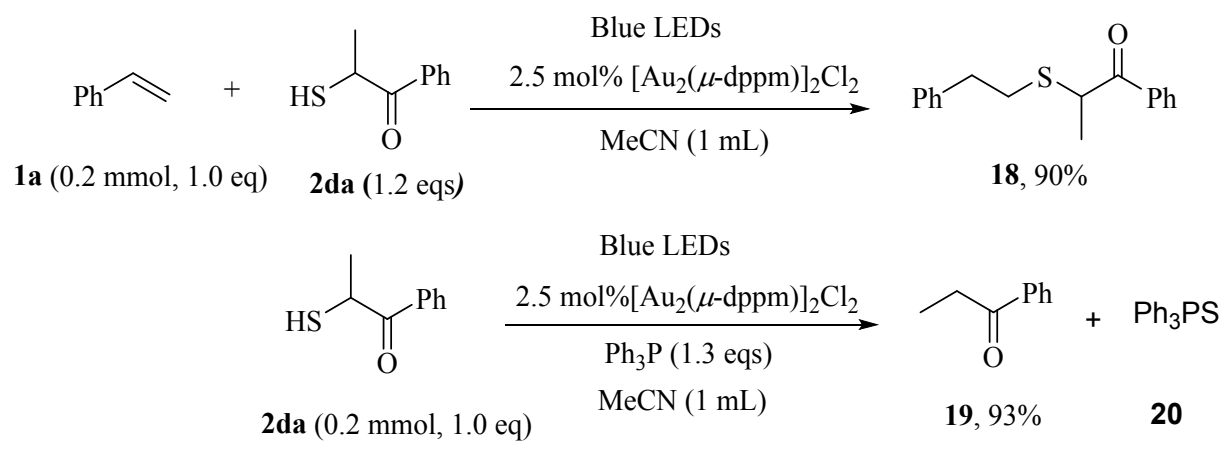

Scheme S3. Control experiments for thio-ene reaction and desulfurizing reaction.

Following the general procedure, on the condition without $\mathrm{Ph}_{3} \mathrm{P}$ (eq. 1) or in the absence of styrene (eq. 2), giving thiol addition and desulfurized products 18 and 19, respectively. In the absence of $\mathrm{Ph}_{3} \mathrm{P}$, the mixture of $\left[\mathrm{Au}_{2}(\mu-\mathrm{dppm})_{2}\right] \mathrm{Cl}_{2}$ with $\mathbf{2 d a}$ produce thiols radical for thio-ene reaction. 
2-(phenethylthio)-1-phenylpropan-1-one 18

Colorless oil; 90\% yield.
IR (Reflection): $\tilde{v}=3650,3335,3085,3061,3027,2970,2928$, 2858, 2408, 1961, 1898, 1812, 1680, 1596, 1581, 1496, 1449, 1372, 1332, 1309, 1234 , 1178, 1100, 1076, 1060, 1029, 1001, 950, 844, 800, 711, 697, 656; ${ }^{1} \mathrm{H}$ NMR (500 MHz, $\left.\mathrm{CDCl}_{3}\right): \delta=8.02(\mathrm{~d}, J=7.4 \mathrm{~Hz}, 2 \mathrm{H}), 7.58(\mathrm{t}, J=7.4 \mathrm{~Hz}, 1 \mathrm{H}), 7.48(\mathrm{t}, J=7.7 \mathrm{~Hz}, 2 \mathrm{H})$, 7.30-7.24 (dd, $J=9.1,5.7 \mathrm{~Hz}, 2 \mathrm{H}), 7.21$ (t, $J=7.3 \mathrm{~Hz}, 1 \mathrm{H}), 7.15(\mathrm{~d}, J=7.2 \mathrm{~Hz}, 2 \mathrm{H})$, $4.36(\mathrm{q}, J=6.9 \mathrm{~Hz}, 1 \mathrm{H}), 2.84-2.76(\mathrm{~m}, 3 \mathrm{H}), 2.74-2.66(\mathrm{~m}, 1 \mathrm{H}), 1.59(\mathrm{~d}, J=6.9 \mathrm{~Hz}$, 3H); ${ }^{13} \mathrm{C} \mathrm{NMR}\left(125 \mathrm{MHz}, \mathrm{CDCl}_{3}\right): \delta=196.2(\mathrm{~s}), 140.3(\mathrm{~s}), 135.7(\mathrm{~s}), 133.2(\mathrm{~d}), 128.7(\mathrm{~d})$, 128.6(d), 128.5(d), 126.5(d), 41.7(d), 36.0(t), 30.3(t), 16.6(q); HRMS (DART) Calcd for $\mathrm{C}_{17} \mathrm{H}_{19} \mathrm{OS}[\mathrm{M}+1]$ : 271.1151, found: 271.1145.

propiophenone $\mathbf{1 9}^{[11]}$

O Colorless oil; 93\% yield.

${ }^{1} \mathrm{H}$ NMR $\left(500 \mathrm{MHz}, \mathrm{CDCl}_{3}\right): \delta=7.99-7.94(\mathrm{~m}, 2 \mathrm{H}), 7.58-7.53(\mathrm{~m}, 1 \mathrm{H})$, $7.46(\mathrm{t}, J=7.7 \mathrm{~Hz}, 2 \mathrm{H}), 3.01(\mathrm{q}, J=7.2 \mathrm{~Hz}, 2 \mathrm{H}), 1.23(\mathrm{t}, J=7.2 \mathrm{~Hz}, 3 \mathrm{H}) ;{ }^{13} \mathrm{C} \mathrm{NMR}$ $\left(125 \mathrm{MHz}, \mathrm{CDCl}_{3}\right): \delta=201.0$ (s), 137.01 (s), 133.0 (d), 128.7 (d), 128.1 (d), 31.9 (t), $8.4(q)$.

triphenylphosphine sulfide $\mathbf{1 4}^{[12]}$

$\mathbf{P h}_{\mathbf{3}} \mathbf{P}=\mathbf{S}$ White solid;

${ }^{1} \mathrm{H}$ NMR $\left(300 \mathrm{MHz}, \mathrm{CDCl}_{3}\right): \delta=7.79-7.66(\mathrm{~m}, 6 \mathrm{H}), 7.55-7.40(\mathrm{~m}, 9 \mathrm{H}) ;{ }^{13} \mathrm{C} \mathrm{NMR}(125$ $\left.\mathrm{MHz}, \mathrm{CDCl}_{3}\right): \delta=133.0(\mathrm{~s}, \mathrm{~d}, J=84.7 \mathrm{~Hz}), 132.4(\mathrm{~d}, J=10.7 \mathrm{~Hz}), 131.7$ (d, $J=3.0$ $\mathrm{Hz}), 128.65$ (d, $J=12.5 \mathrm{~Hz}) ;{ }^{31} \mathrm{P} \mathrm{NMR}\left(122 \mathrm{MHz}, \mathrm{CDCl}_{3}\right): \delta=43.32$; 


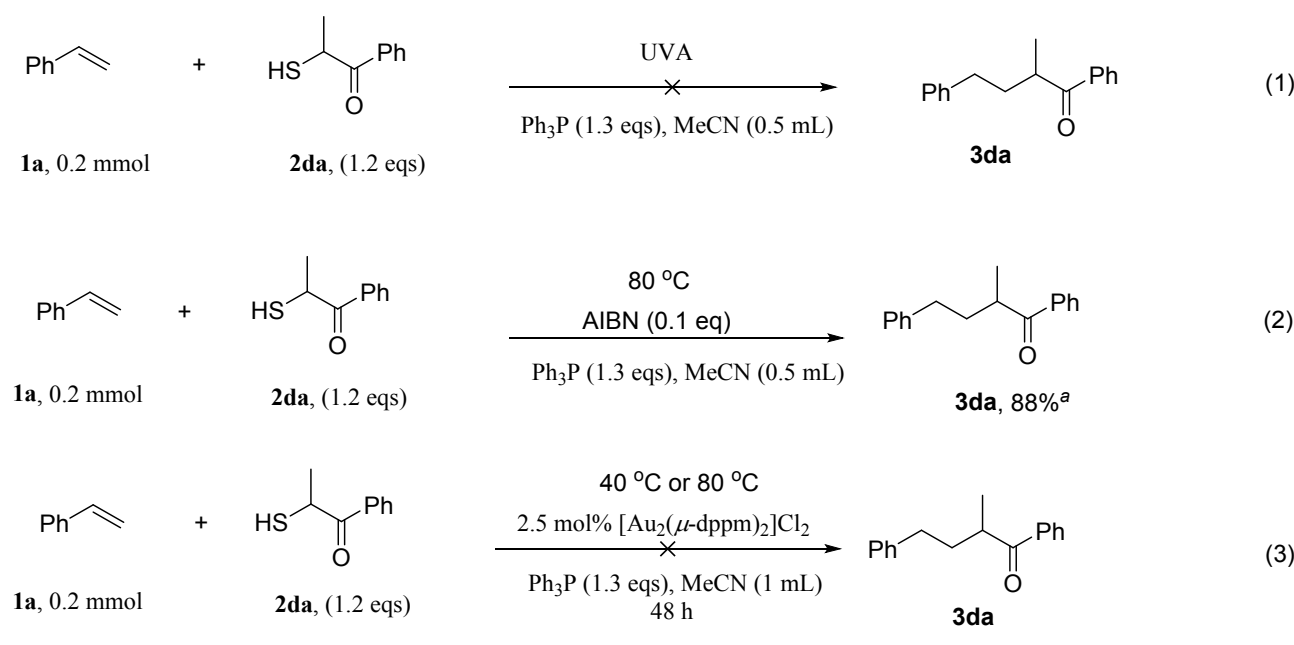

Scheme S4. Control experiments for other energy sources.

Without gold catalyst, no desired product were produced even irradiated by UVA light (eq. 1). By using $\mathrm{AIBN}$ as initiator at $80{ }^{\circ} \mathrm{C}$ and $88 \%$ yield of product were formed which means radical chain progress could be involved in the reaction (eq. 2), however, it is worth to mention that the photoredox path cannot be excluded base on the quantum yield is 0.195 . One reasonable explanation is that the radical chain propagation could be very efficient at high temperature, however at room temperature, the radical chain propagation reaction is not efficient enough which need the photoredox catalysis by gold catalyst in order to get the high yield. Initiating with AIBN at $80{ }^{\circ} \mathrm{C}$ would be an alternative path we are providing to synthesis the same product in the condition of substrates and products are thermal-stable. In order to exclude the possibility of intermediate reacting with the heat of the light, we proceed the reaction under heating at $40{ }^{\circ} \mathrm{C}$ and $80{ }^{\circ} \mathrm{C}$ without blue leds irradiation (eq. 3). The results showed that replace blue led light with heating, no reaction occurred. 


\section{e) Time profile}

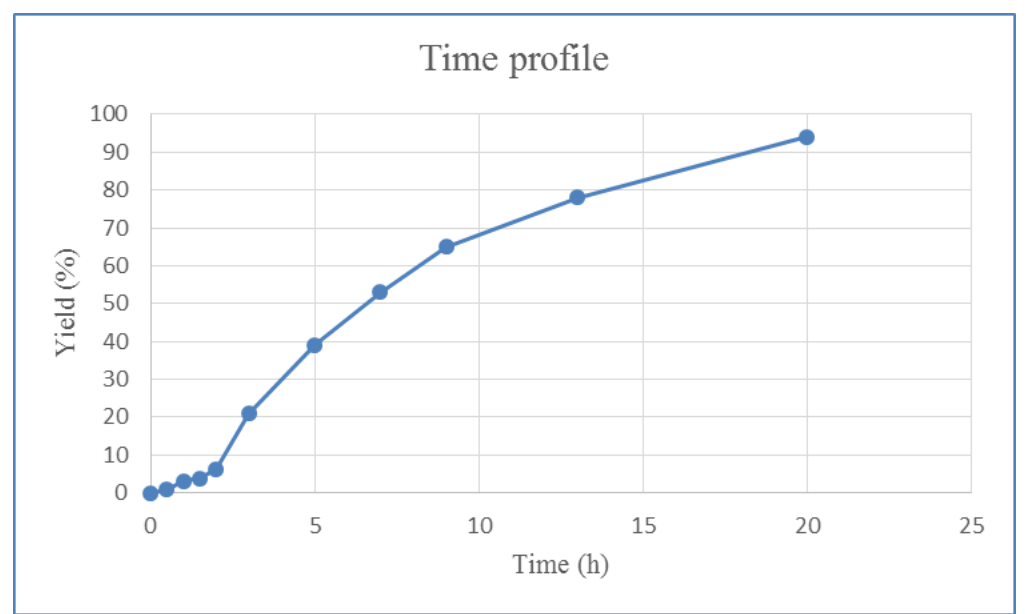

Time profile for react $\mathbf{2 a}$ with styrene under the optimal condition shows that there is an inducing time at the beginning of 2 hours.

\section{f) UV-vis spectra}

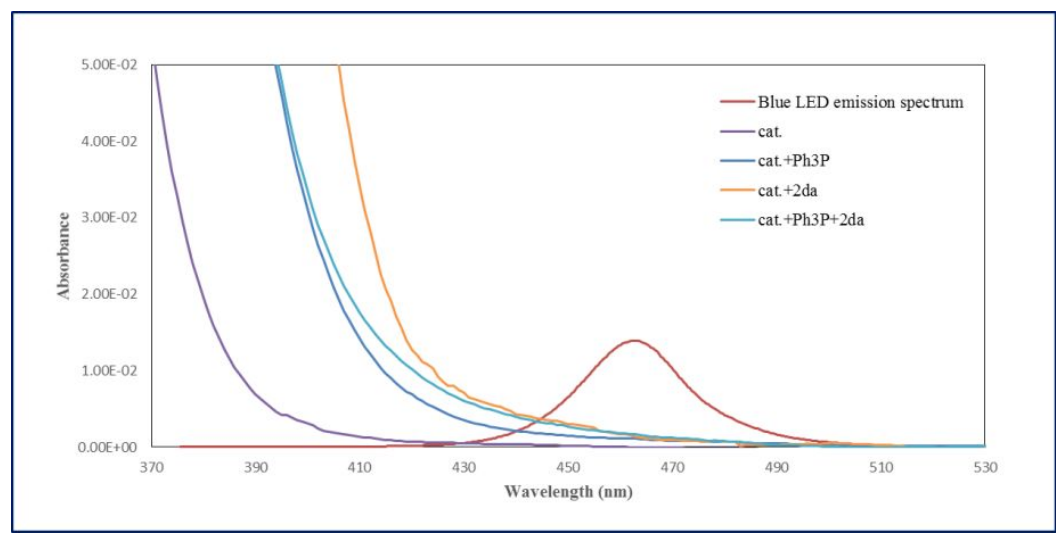

$\left[\mathrm{Au}_{2}(\mu-\mathrm{dppm})_{2}\right] \mathrm{Cl}_{2}$

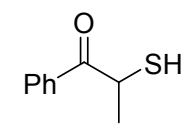

cat.

2da

\section{Method}

(a) Only $\left[\mathrm{Au}_{2}(\mu \text {-dppm })_{2}\right] \mathrm{Cl}_{2}: 2.5 \times 10^{-5} \mathrm{~mol} / \mathrm{mL}\left[\mathrm{Au}_{2}(\mu \text {-dppm })_{2}\right] \mathrm{Cl}_{2}$ in $\mathrm{MeCN}$.

(b) $\left[\mathrm{Au}_{2}(\mu-\mathrm{dppm})_{2}\right] \mathrm{Cl}_{2}+\mathrm{Ph}_{3} \mathrm{P}$ or $2 \mathrm{da}:\left[\mathrm{Au}_{2}(\mu-\mathrm{dppm})_{2}\right] \mathrm{Cl}_{2}(0.025 \mathrm{mmol})$ with $\mathrm{Ph}_{3} \mathrm{P}$ or thio $(0.26 \mathrm{mmol})$ in $\mathrm{MeCN}(2 \mathrm{~mL})$ in glove-box, room temperature. After 1h stirring, the insoluble solid were isolated by filter, then measure the UV-vis spectrum of the 
solution.

The UV-vis absorption spectrum showed that the $\left[\mathrm{Au}_{2}(\mu \text {-dppm })_{2}\right] \mathrm{Cl}_{2}$ don't absorb the light in the emission wavelength of blue led. However, it has obviously red shift when we measured the mixture under $\mathrm{N}_{2}$ atmosphere, an overlap between blue led emission spectrum and $\left[\mathrm{Au}_{2}(\mu-\mathrm{dppm})_{2}\right] \mathrm{Cl}_{2}+\mathrm{Ph}_{3} \mathrm{P}$ (with $\mathrm{N}_{2}$ protected) absorption or $\left[\mathrm{Au}_{2}(\mu-\right.$ dppm) $\left.{ }_{2}\right] \mathrm{Cl}_{2}+$ thio $2 \mathrm{da}$ (with $\mathrm{N}_{2}$ protected) spectrum observed. Besides, the mixture of $\left[\mathrm{Au}_{2}(\mu \text {-dppm })_{2}\right] \mathrm{Cl}_{2}+\mathrm{Ph}_{3} \mathrm{P}+$ mercaptan $(2 \mathrm{da})$ under with $\mathrm{N}_{2}$ protected also gave a bathochromic shift compared to $\left[\mathrm{Au}_{2}(\mu-\mathrm{dppm})_{2}\right] \mathrm{Cl}_{2}+\mathrm{Ph}_{3} \mathrm{P}$. These results provides a highly possibility that the real photosensitizer is the newly gold complex formed at the beginning of the reaction. 


\section{g) Quantum yield}

The quantum yield $(\Phi)$ was determined by the known ferrioxolate actinometry method. A ferrioxolate actinometry solution was prepared by following the Hammond variation of the Hatchard and Parker procedure outlined in the Handbook of Photochemistry. ${ }^{[13]}$ The irradiated light intensity was estimated to $3.00 \times 10-7$ Einstein $\mathrm{S}^{-1}$ by using $\mathrm{K}_{3}\left[\mathrm{Fe}\left(\mathrm{C}_{2} \mathrm{O}_{4}\right)_{3}\right]$ as an actinometer.

Five dried Pyrex screw-top reaction tubes were each charged with $\left[\mathrm{Au}_{2}(\mu \text {-dppm })_{2}\right] \mathrm{Cl}_{2}$ (2.5 mol \%), $\mathrm{Ph}_{3} \mathrm{P}(1.3 \mathrm{~N})$ was dissolved in $\mathrm{MeCN}(0.5 \mathrm{~mL})$. Then the alkenes 1a $(0.1$ mmol, $1 N)$, the mercaptans $2 \mathrm{a}(1.2 N)$ were added. The reaction mixture was then cooled to $-78^{\circ} \mathrm{C}$ and degassed via vacuum evacuation ( $5 \mathrm{~min}$ ), backfilled with nitrogen, and warmed to room temperature. This process was repeated three times. After the reaction was thoroughly degassed, the solutions were irradiation with Blue LEDs for specified time intervals ( $0 \mathrm{~min}, 30 \mathrm{mins}, 60 \mathrm{mins}, 90 \mathrm{mins}, 120 \mathrm{mins})$. The moles of products formed were determined by GC-MS with dodecane as reference standard. The number of moles of products (y axis) per unit time is related to the number of photons (x axis, calculated from the light intensity). The slope of the graph represented in Figure equals the quantum yield $(\Phi)$ of the photoreaction. $\Phi=19.5 \%$

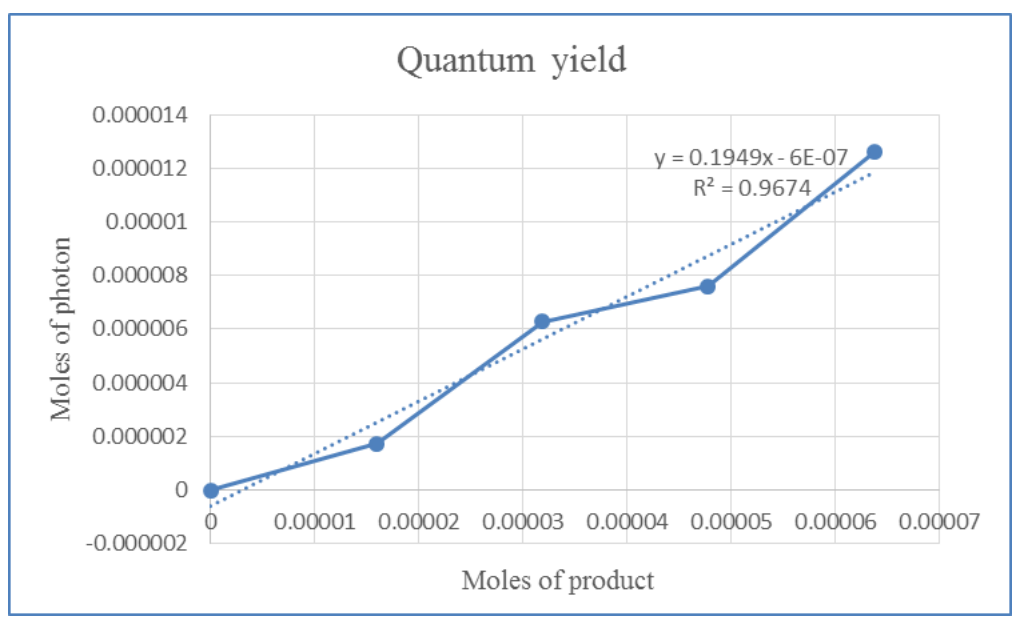


h) Oxidation $\mathrm{Ph}_{3} \mathrm{P}$ with $\mathrm{O}_{2}$ in the presence of $\left[\mathrm{Au}_{2}(\mu \text {-dppm })_{2}\right] \mathrm{Cl}_{2}$
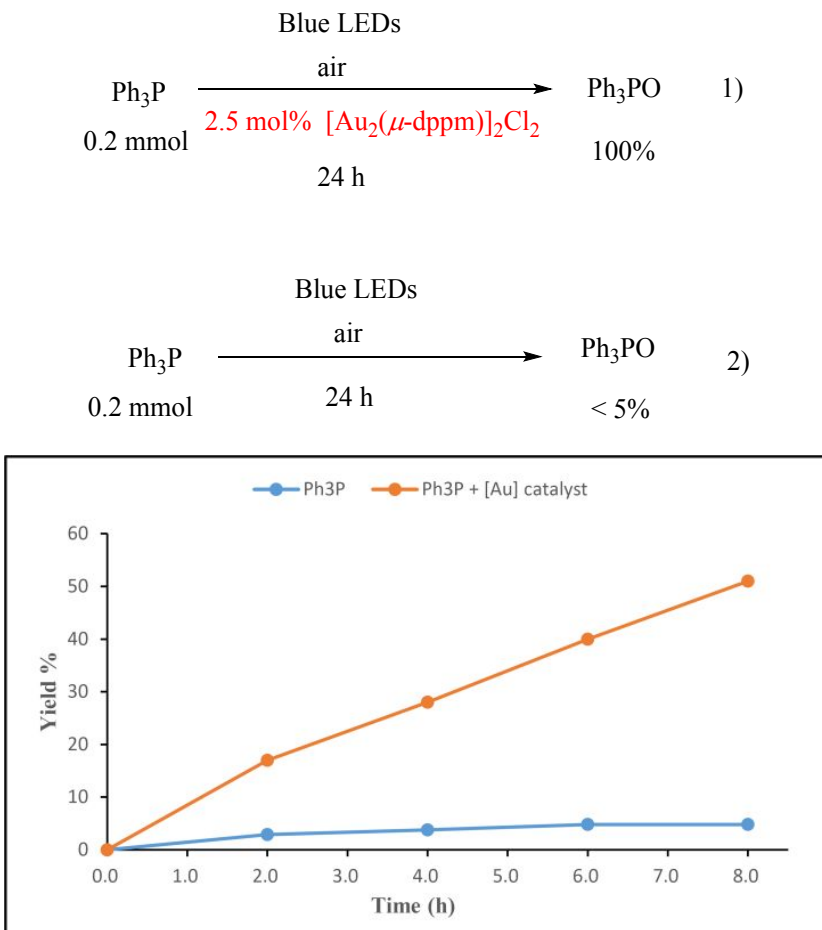

The oxidation of triphenylphosphine in the presence of catalytic amount of gold catalyst and air has processed well, in the case of without gold catalyst, only trace amount of $\mathrm{Ph}_{3} \mathrm{PO}$ were formed. 
i) Oxidation $\mathrm{Ph}_{3} \mathrm{P}$ with 4-iodoanisole in the presence of $\left[\mathrm{Au}_{2}(\mu \text {-dppm })_{2}\right] \mathrm{Cl}_{2}$
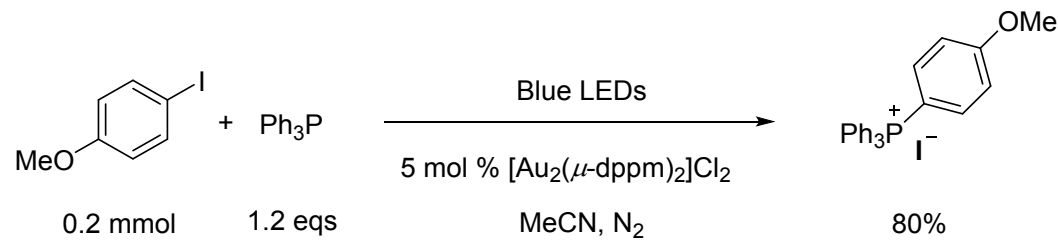

The oxidation of triphenylphosphine with 4-iodoanisole in the presence of substoichiometric amount of gold catalyst has processed well.

\section{j) Stoichiometric experiment and NMR identification}

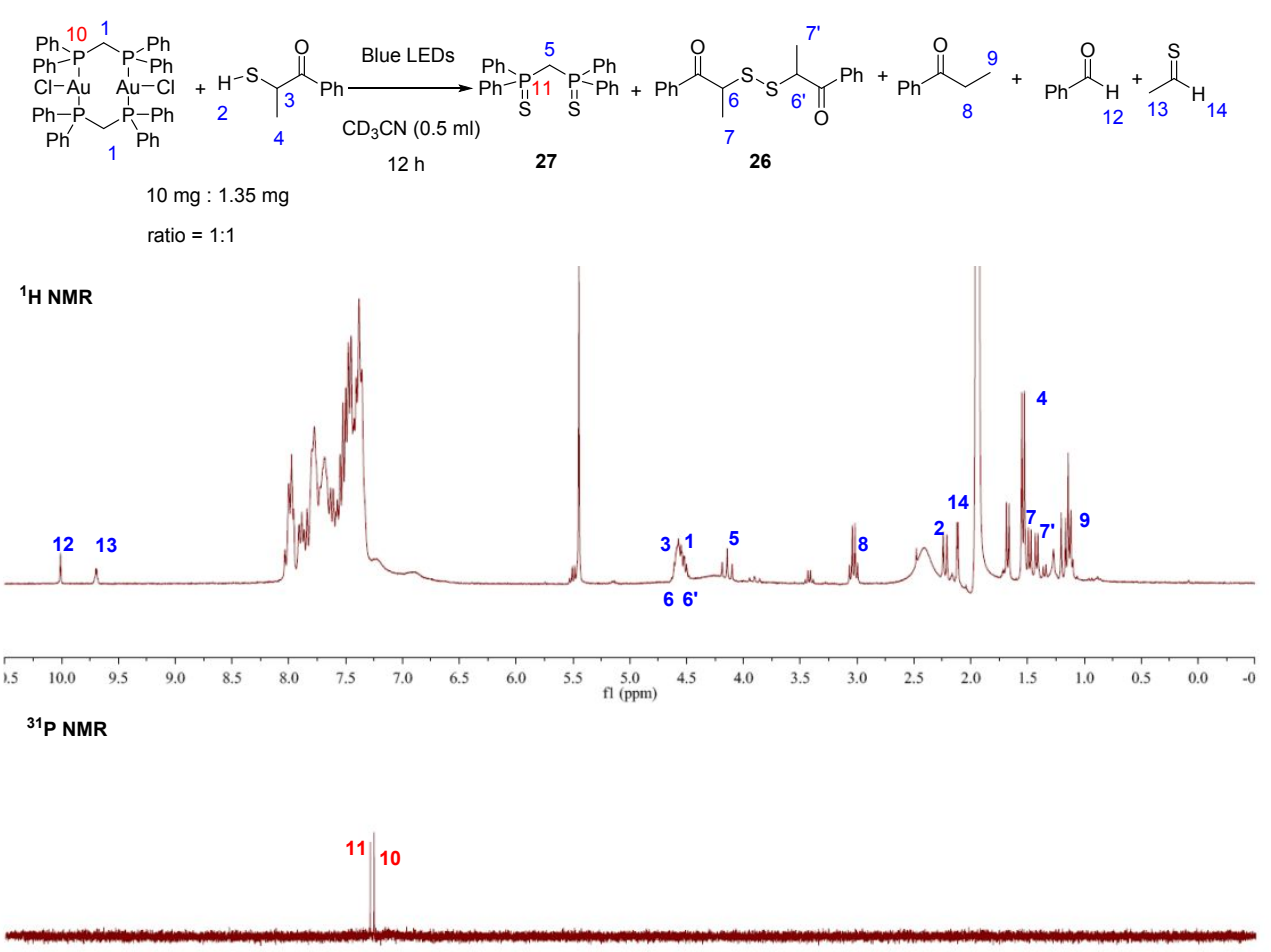

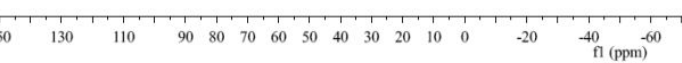

Figure S3a. NMR identification in $\mathrm{CD}_{3} \mathrm{CN}$ (gold catalyst : thio $=1: 1$ ) 

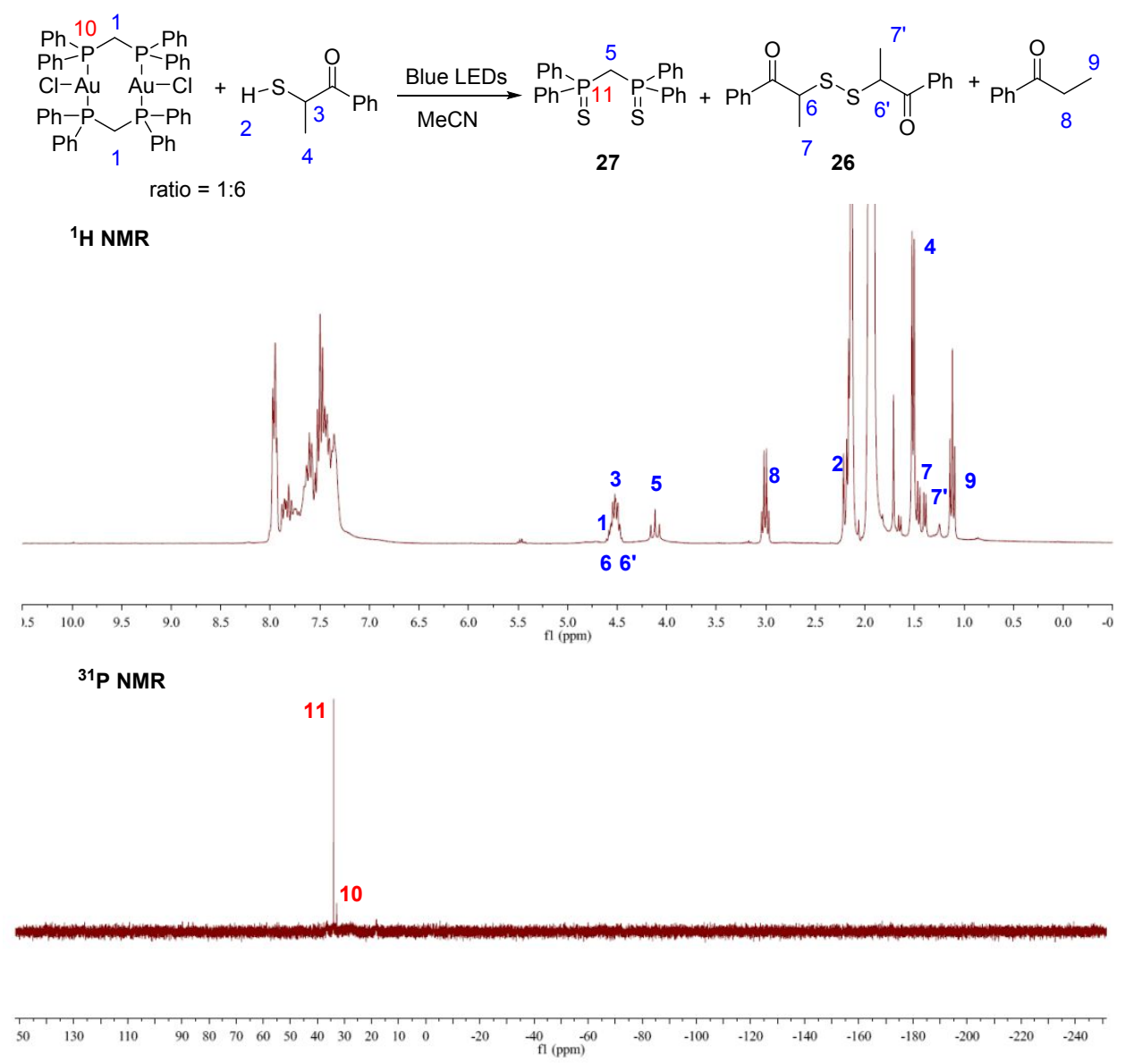

Figure S3b. NMR identification in $\mathrm{CD}_{3} \mathrm{CN}$ (gold catalyst : thio =1:6).

The stoichiometric experiment indicate that thiyl radical would be formed by irradiating the mixture of $\left[\mathrm{Au}_{2}(\mu-\mathrm{dppm})_{2}\right] \mathrm{Cl}_{2}+2 \mathrm{da}$, which would further converted to desulfurized product, benzaldehyde, and the disulfide product (Figure S3a). The benzaldehyde and ethanethial has been removed after evaporation under vacuum (Figure S3b).

2,2'-disulfanediylbis(1-phenylpropan-1-one) 26<smiles>CC(SSC(C)C(=O)c1ccccc1)C(=O)c1ccccc1</smiles>

26 , isomer 1
Colorless oil.

Isomer 1: ${ }^{1} \mathrm{H} \mathrm{NMR}\left(300 \mathrm{MHz}, \mathrm{CDCl}_{3}\right): \delta=8.03-7.99(\mathrm{~m}, 4 \mathrm{H})$, 7.64-7.58 (m, 2H), 7.58-7.54 (m, 4H), 4.48 (q, $J=6.8 \mathrm{~Hz}, 2 \mathrm{H})$,

$1.48(\mathrm{~d}, \mathrm{~J}=6.8 \mathrm{~Hz}, 6 \mathrm{H}) ;{ }^{13} \mathrm{C} \mathrm{NMR}\left(101 \mathrm{MHz}, \mathrm{CDCl}_{3}\right): \delta=197.1(\mathrm{~s}), 135.6(\mathrm{~s}), 133.7(\mathrm{~d})$, 129.0(d), 128.9(d), 48.0(d), 16.6(q). HRMS (EI) $\mathrm{C}_{18} \mathrm{H}_{18} \mathrm{O}_{2} \mathrm{~S}_{2}\left[\mathrm{M}^{\cdot+}\right]$ Calcd for 330.07427, found: 330.07341 . 
2,2'-disulfanediylbis(1-phenylpropan-1-one) $\mathbf{2 6}$<smiles>CC(SSC(C)C(=O)c1ccccc1)C(=O)c1ccccc1</smiles>

Colorless oil.

Isomer 2: ${ }^{1} \mathrm{H} \mathrm{NMR}\left(300 \mathrm{MHz}, \mathrm{CDCl}_{3}\right): \delta=7.84-7.80(\mathrm{~m}, 4 \mathrm{H})$,

26 , isomer 2 7.56-7.50 (m, 2H), 7.40-7.35 (m, 4H), 4.39 (q, $J=6.8 \mathrm{~Hz}, 2 \mathrm{H})$, $1.57(\mathrm{~d}, J=6.9 \mathrm{~Hz}, 6 \mathrm{H}) ;{ }^{13} \mathrm{C} \mathrm{NMR}\left(101 \mathrm{MHz}, \mathrm{CDCl}_{3}\right): \delta=197.1(\mathrm{~s}), 135.6(\mathrm{~s}), 133.5(\mathrm{~d})$, 128.9(d), 128.8(d), 48.3(d), 17.0(q). HRMS (EI) $\mathrm{C}_{18} \mathrm{H}_{18} \mathrm{O}_{2} \mathrm{~S}_{2}\left[\mathrm{M}^{\cdot+}\right]$ Calcd for 330.07427, found: 330.07353 .

methylenebis(diphenylphosphine sulfide) 27

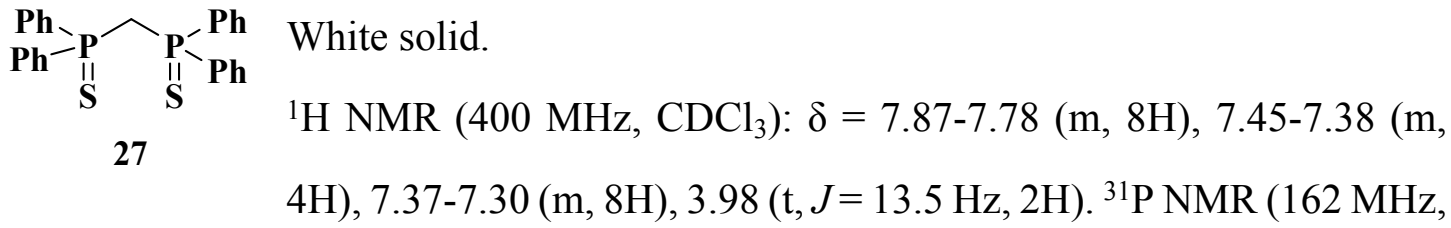
$\left.\mathrm{CDCl}_{3}\right): \delta=35.34(\mathrm{~s}) \cdot \mathrm{HRMS}(\mathrm{ESI}) \mathrm{C}_{25} \mathrm{H}_{22} \mathrm{NaP}_{2} \mathrm{~S}_{2}\left[\mathrm{M}+\mathrm{Na}^{+}\right]$Calcd for 471.0536, found: 471.0533 .

k) Visualized photoreaction

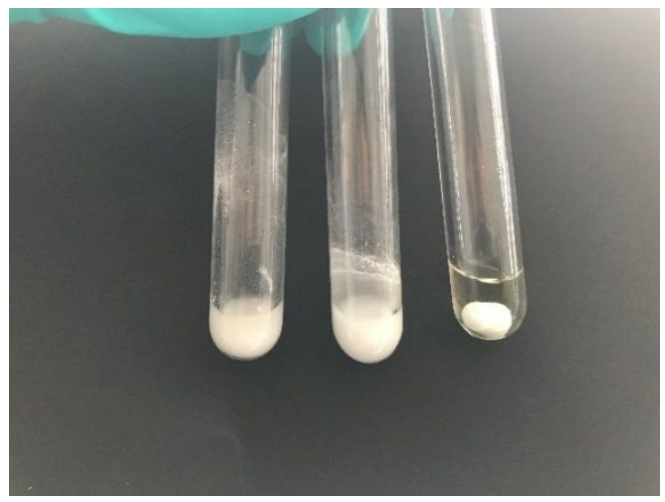

Left: $\left[\mathrm{Au}_{2}(\mu \text {-dppm })_{2}\right] \mathrm{Cl}_{2}(10 \mathrm{mg}, 1.0 \mathrm{eq})+\mathbf{2 d a}(6.0$ eqs $)$ in $\mathrm{MeCN}(1.0 \mathrm{~mL})$ in dark, $12 \mathrm{~h}$.

Middle: $\left[\mathrm{Au}_{2}(\mu-\mathrm{dppm})_{2}\right] \mathrm{Cl}_{2}(10 \mathrm{mg})$ in $\mathrm{MeCN}(1.0 \mathrm{~mL})$ under Blue LEDs, $12 \mathrm{~h}$.

Right: $\left[\mathrm{Au}_{2}(\mu-\mathrm{dppm})_{2}\right] \mathrm{Cl}_{2}(10 \mathrm{mg}, 1.0$ eq $)+\mathbf{2 d a}(6.0$ eqs $)$ in $\mathrm{MeCN}(1.0 \mathrm{~mL})$ under Blue LEDs, $12 \mathrm{~h}$. 
After irradiating the mixture of $\left[\mathrm{Au}_{2}(\mu-\mathrm{dppm})_{2}\right] \mathrm{Cl}_{2}+$ mercaptan (2da) under Blue LEDs, the reaction mixture turned to light-yellow homogeneous solution. However, in the dark or in the absence of mercaptan show much insoluble $\left[\mathrm{Au}_{2}(\mu-\mathrm{dppm})_{2}\right] \mathrm{Cl}_{2}$ solid exist. Besides, no reaction has happened in the condition of $2 \mathrm{da}$ in $\mathrm{CD} 3 \mathrm{CN}$ under Blue LEDs. 
I) Detection newly formed gold complex by NMR spetra

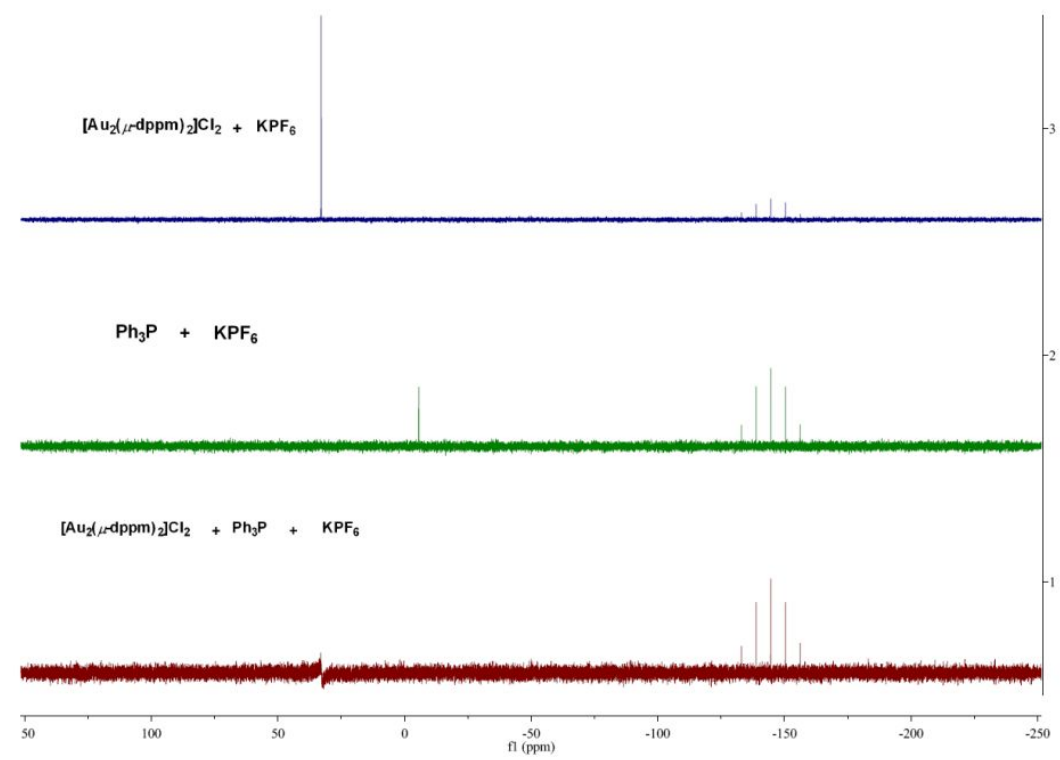

Figure S4a. ${ }^{31} \mathrm{P}$ NMR spectrums in $\mathrm{CD}_{3} \mathrm{CN}$

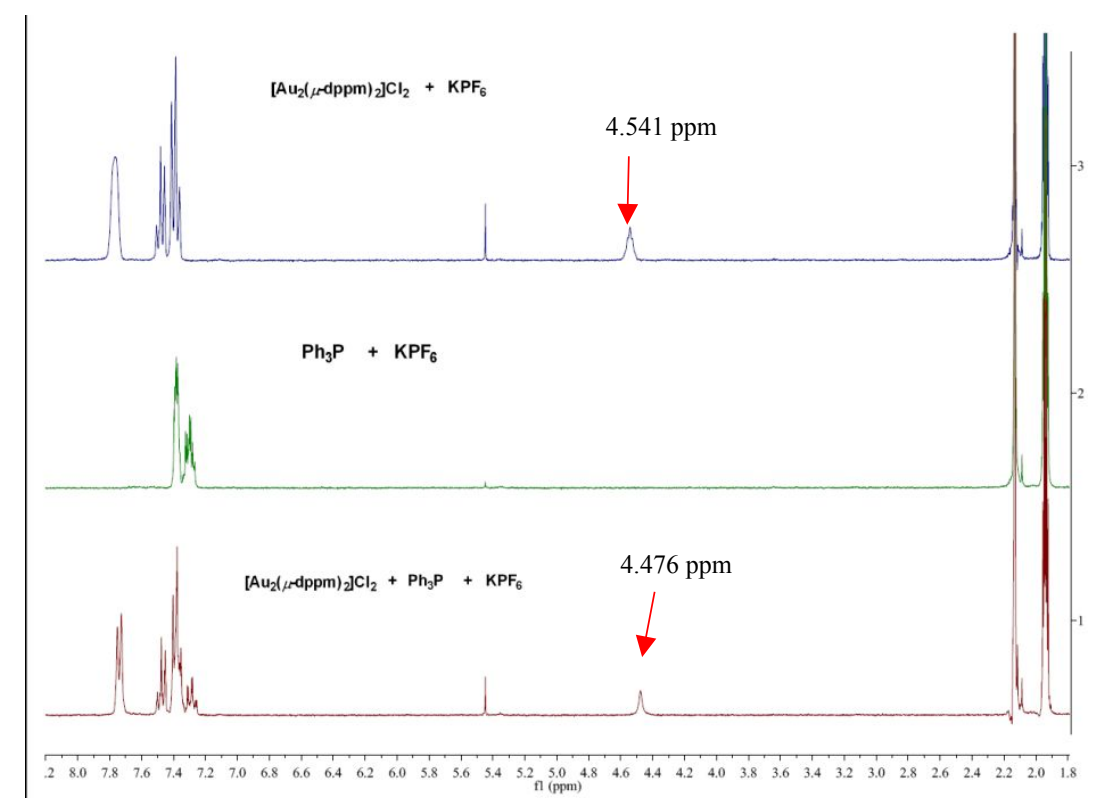

Figure S4b. ${ }^{1} \mathrm{H}$ NMR spectrums in $\mathrm{CD}_{3} \mathrm{CN}$

\section{From top to bottom (figure S4a):}

1) The mixture of $\left[\mathrm{Au}_{2}(\mu-\mathrm{dppm})_{2}\right] \mathrm{Cl}_{2}\left(2 \times 10^{-3} \mathrm{mmol}\right)$ and $\mathrm{KPF}_{6}\left(2 \times 10^{-3} \mathrm{mmol}\right)$ in $\mathrm{CD}_{3} \mathrm{CN}(0.5 \mathrm{~mL}), \mathrm{N}_{2}$. 
${ }^{31}$ P NMR peaks: $33.011 \mathrm{ppm}(\mathrm{s}),-144.500 \mathrm{ppm}(\mathrm{m})$

2) The mixture of $\mathrm{Ph}_{3} \mathrm{P}\left(2 \times 10^{-3} \mathrm{mmol}\right)$ and $\mathrm{KPF}_{6}\left(2 \times 10^{-3} \mathrm{mmol}\right)$ in $\mathrm{CD}_{3} \mathrm{CN}(0.5 \mathrm{~mL})$, $\mathrm{N}_{2}$.

${ }^{31}$ P NMR peaks: $-5.553 \mathrm{ppm}(\mathrm{s}),-144.500 \mathrm{ppm}(\mathrm{m})$

3) The combination of 1) and 2), stir $30 \mathrm{~min}$ at r.t. under $\mathrm{N}_{2}$.

${ }^{31}$ P NMR peaks: 33.225 ppm (br), -144.500 ppm (m)

Analysis: After stirring $1 \mathrm{~h}$ under $\mathrm{N}_{2}$ atmosphere, ${ }^{31} \mathrm{P}$ NMR peak of $\mathrm{Ph}_{3} \mathrm{P}(-5.553 \mathrm{ppm})$ is disappeared and a broad peak appeared at $33.225 \mathrm{ppm}$ which means new gold complex has been formed by combination of $\left[\mathrm{Au}_{2}(\mu-\mathrm{dppm})_{2}\right] \mathrm{Cl}_{2}$ with $\mathrm{Ph}_{3} \mathrm{P}$ (figure $\mathrm{S} 4 \mathrm{a}$ ), the broad peak would be caused by the equilibrium of two ligand (dppm and $\left.\mathrm{Ph}_{3} \mathrm{P}\right)$. In the ${ }^{1} \mathrm{H}$ NMR, the $\mathrm{CH}_{2}$ peak of dppm changed from 4.541ppm to 4.476ppm (figure $\mathrm{S} 4 \mathrm{~b}$ )

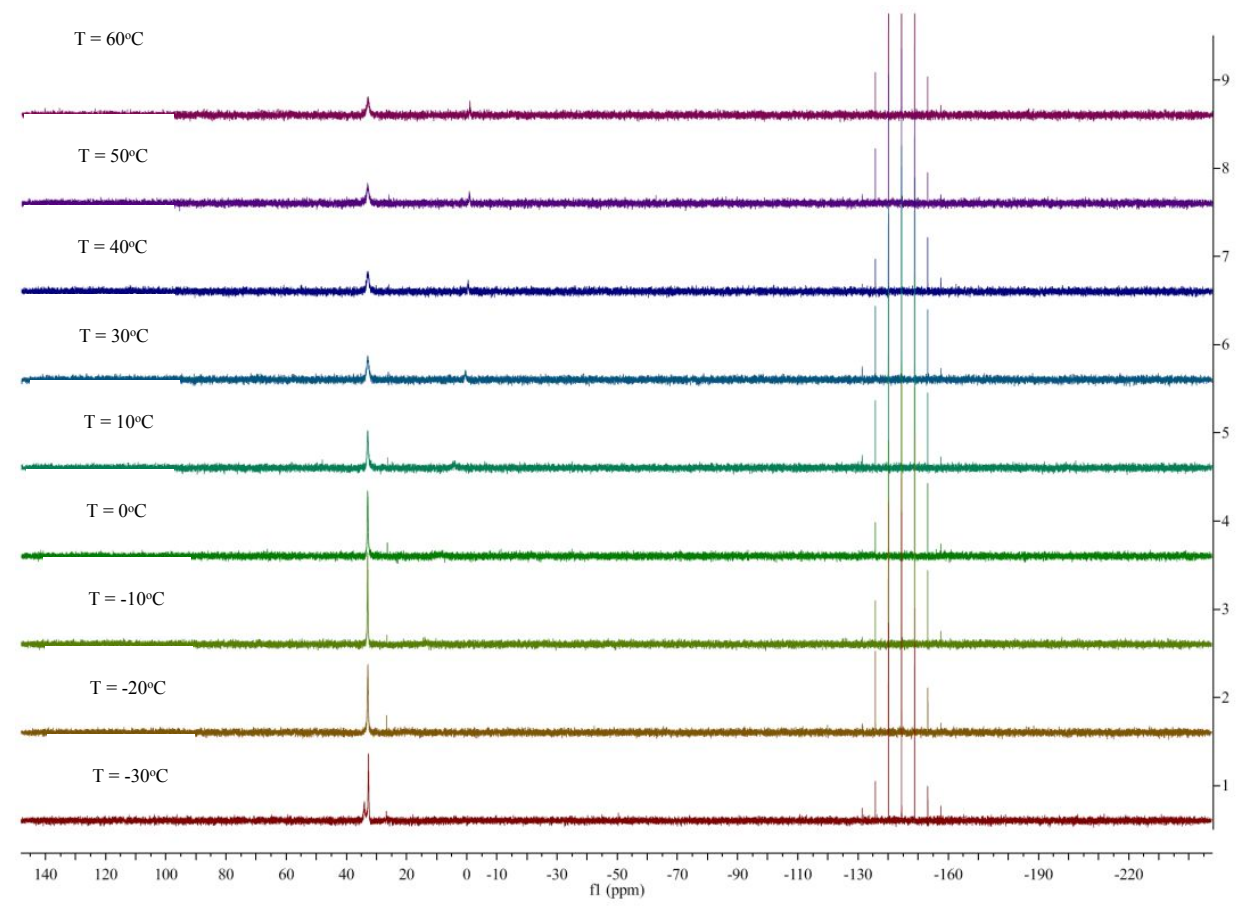

Figure S5a. ${ }^{31} \mathrm{P}$ NMR spectrums in $\mathrm{CD}_{3} \mathrm{CN}$ at different temperature 


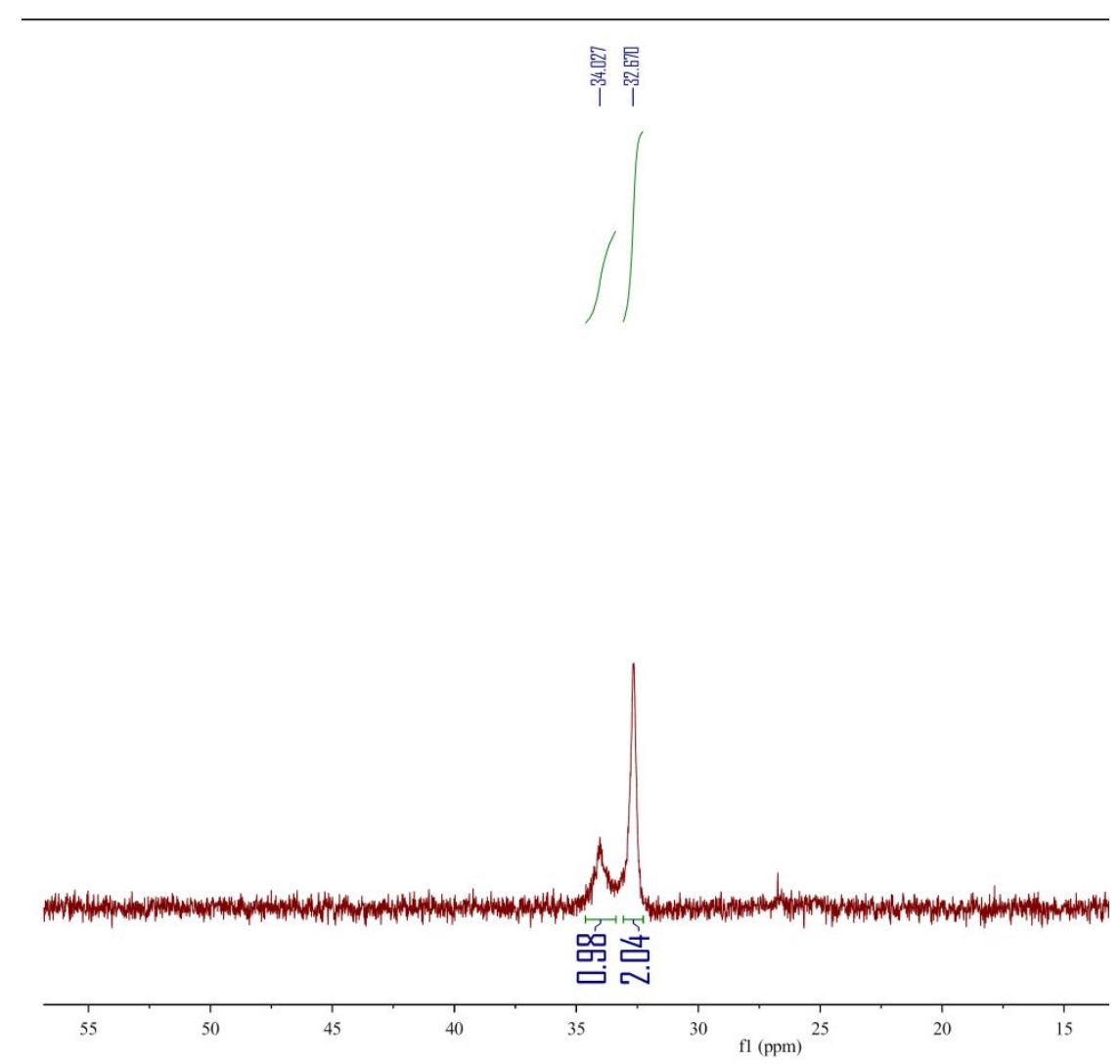

Figure S5b. Local magnification ${ }^{31} \mathrm{P}$ NMR of $-30{ }^{\circ} \mathrm{C}$

\section{Analysis:}

The mixture of $\left[\mathrm{Au}_{2}(\mu \text {-dppm })_{2}\right] \mathrm{Cl}_{2}\left(2 \times 10^{-3} \mathrm{mmol}\right), \mathrm{Ph}_{3} \mathrm{P}\left(2 \times 10^{-3} \mathrm{mmol}\right)$ and $\mathrm{KPF}_{6}\left(4 \times 10^{-}\right.$ $\left.{ }^{3} \mathrm{mmol}\right)$ in $\mathrm{CD}_{3} \mathrm{CN}(1.0 \mathrm{~mL}), \mathrm{N}_{2}$.

${ }^{31} \mathrm{P}$ NMR peak standard $\left(\mathrm{KPF}_{6}\right):-144.500 \mathrm{ppm}(\mathrm{m})$

a) Two peak formed obviously at $-30{ }^{\circ} \mathrm{C}$ at $-32.670 \mathrm{ppm}$ and $-34.027 \mathrm{ppm}$ (ratio $=2: 1$ ) would be caused by lower equilibrium rate of different $\mathrm{P}$ ligand in low temperature.

b) The shape of ${ }^{31} \mathrm{P}$ NMR peak changed from broaden to sharp by decreasing the temperature. 
m) Initiating the reaction with different wavelength of lights by filter control.

\begin{tabular}{|c|c|c|c|}
\hline $\begin{array}{l}\mathrm{Ph} \curvearrowright \\
0.2 \mathrm{mmol}\end{array}$ & $\prod_{\mathrm{O}}^{\mathrm{Ph}}$ & $\begin{array}{c}\mathrm{hv} \text { (light filter) } \\
2.5 \mathrm{~mol} \%\left[\mathrm{Au}_{2}(\mu \text {-dppm })_{2}\right] \mathrm{Cl}_{2} \\
\underset{\mathrm{Ph}_{3} \mathrm{P}(1.3 \text { eqs }), \mathrm{MeCN}(0.5 \mathrm{~mL})}{\longrightarrow}\end{array}$ & O \\
\hline wavelength of light filiter & & time & yield $^{a}$ \\
\hline$\lambda>485 \mathrm{~nm}$ & & $0.5 \mathrm{~h}$ & n.r. \\
\hline$\lambda>435 \mathrm{~nm}$ & & $0.5 \mathrm{~h}$ & $16 \%$ \\
\hline$\lambda>435 \mathrm{~nm}$ & & $6.0 \mathrm{~h}$ & $80 \%$ \\
\hline
\end{tabular}

In order to confirm the photosensitizer absorb light in the wavelength of blue led, we proceed the reaction by using light filter. The results showed that irradiated the reaction with wavelength above $485 \mathrm{~nm}$ light, no product formed. However, when irradiated the reaction with wavelength above $435 \mathrm{~nm}$ light, $16 \%$ yield product produced and extend the reaction time for 6 hours, $80 \%$ yield of product formed. This would highly prove that the reaction could proceed by absorbing blue led light.

\section{n) Using phosphate buffer solution as only solvent}

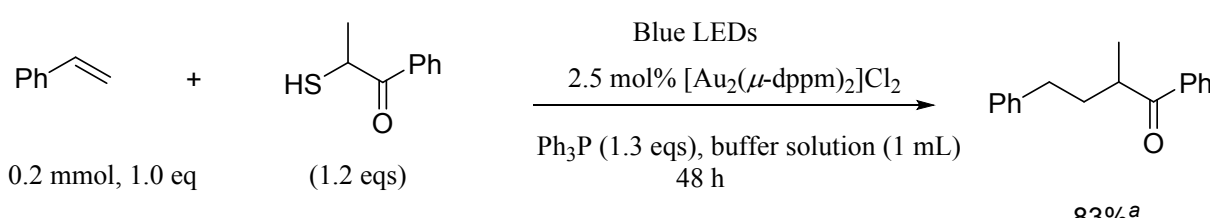

The reaction proceed only in phosphate buffer solution can get the desire product in $83 \%$ yield which would provide the possibility of using this methodology in biological compatible system. 
o) Gram scale synthesis

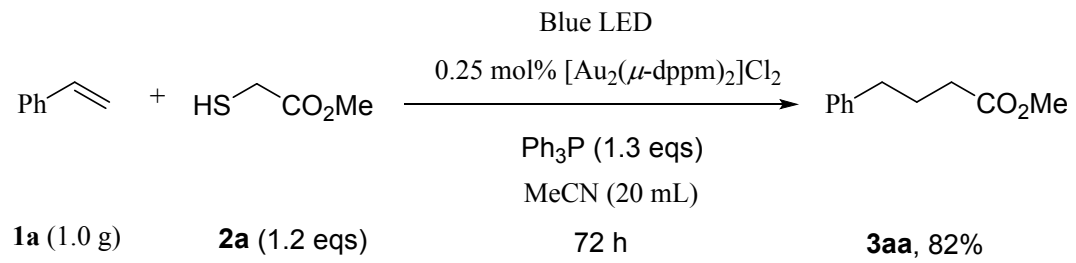

By following the general procedure, gram scale synthesis of $\mathbf{3 a a}$ is successful in the condition of $0.25 \mathrm{~mol} \%\left[\mathrm{Au}_{2}(\mu-\mathrm{dppm})_{2}\right] \mathrm{Cl}_{2}$ and $72 \mathrm{~h}$ reaction time.

p) Attempt with an unactivated aliphatic alkene as coupling partner

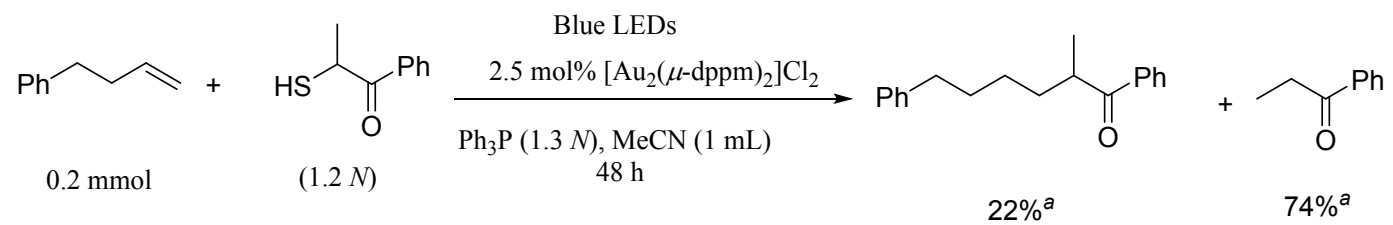

For unactivated aliphatic alkene, only $22 \%$ yield of desulfurized coupling product were formed along with yielding $74 \%$ desulfurized product. This result indicate that the electron-poor alkyl radical which resulting from desulfurizing of thio is not competitive to coupling with unactivated aliphatic alkene compare with hydrogen abstraction from thios. 
q) Failure attempts for reacting methyl bromoacetate with styrene

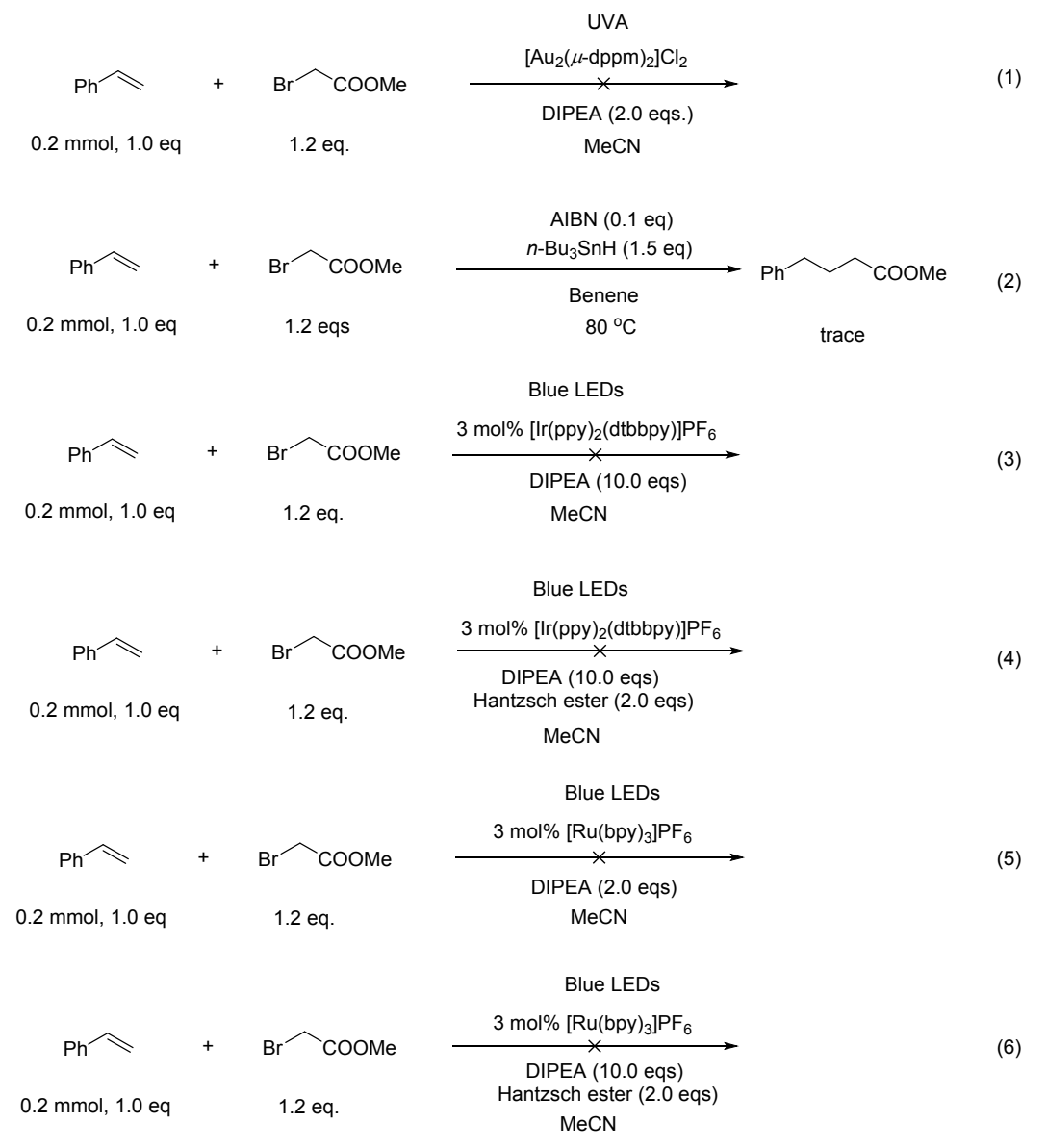

The attempts to synthesis the same product from methyl bromoacetate were all failed by following reported $[\mathrm{Ir}]^{15},[\mathrm{Ru}]^{16},\left[\mathrm{Au}_{2}\right]^{17}$ systems and even classic Giese reaction condition. 
VI. X-ray structure of 25

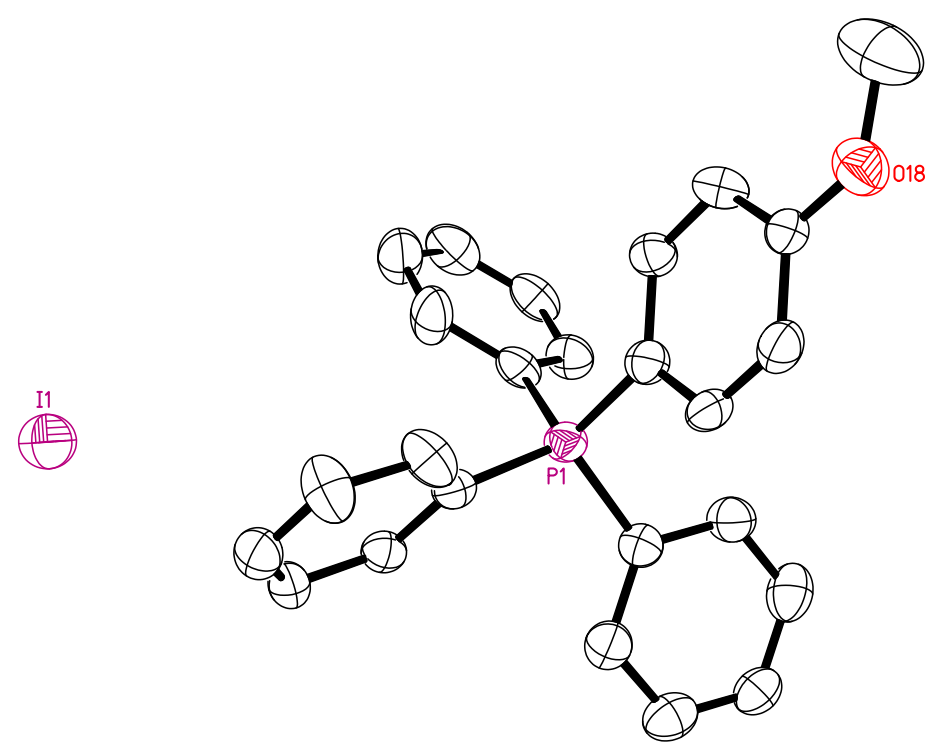

Compound 25 


\section{References}

[1] Bharate, S. B.; Mudududdla, R.; Sharma, R.; Vishwakarma, R. A. The First Method for C-Devinylation of Aromatic Systems. Tetrahedron Lett. 2013, 54, 2913-2915.

[2] Selby, T. M.; Clarkson, J. R.; Mitchell, D.; Fitzpatrick, J. A. J.; Lee, H. D.; Pratt D. W.; Zwier T. S. Isomer-Specific Spectroscopy and Conformational Isomerization Energetics of o-, m-, and p-Ethynylstyrenes. J. Phys. Chem. A, 2005, 109, $4484-4496$.

[3] Crudden, C. M.; Ziebenhaus, C.; Rygus, J. P. G.; Ghozati, K.; Unsworth, P. J.; Nambo, M.; Voth, S.; Hutchinson, M.; Laberge, V. S.; Maekawa, Y.; Imao, D. Iterative Protecting Group-Free Cross-Coupling Leading to Chiral Multiply Arylated Structures. Nat. Commun. 2016, 7, 1-7.

[4] Molander, G. A.; Brown, A. R. Suzuki-Miyaura Cross-Coupling Reactions of Potassium Vinyltrifluoroborate with Aryl and Heteroaryl Electrophiles. J. Org. Chem. 2006, 71, 9681-9686.

[5] Fang, X. J.; Yu, P.; Cerai, G. P.; Morandi, B. Unlocking Mizoroki-Heck-Type Reactions of Aryl Cyanides Using Transfer Hydrocyanation as a TurnoverEnabling Step. Chem. Eur. J. 2016, 22, 15629-15633.

[6] Hatanaka, T.; Yuki, R.; Saito, R.; Sasaki, K. $\alpha$-Methylphenacyl Thioesters as Convenient Thioacid Precursors. Org. Biomol. Chem. 2016, 14, 10589-10592.

[7] Gaumont, A. C.; Waznbh, L.; Denis, J. M. Thiocyanohydrins, A New Class of Compounds, Precursors of Unstabilized Thiocarbonyl Derivatives. Tetrahedron 1991, 47, 4927-4940.

[8] Kraus, G. A.; Bae, J.; Choudhury, P. K. A Direct Introduction of the Mercaptoacetic Acid Unit into Amino Acid Esters. Synthesis 2003, 1, 19-20.

[9] Yusof, Y.; Tan, D. T. C.; Arjomandi, O. K.; Schenk, G.; McGeary, R. P. Captopril Analogues as Metallo- $\beta$-Lactamase Inhibitors. Bioorg. Med. Chem. Lett. 2016, 26, $1589-1593$.

[10] Ludwiczak, M.; Bayda, M.; Dutkiewicz, M.; Frąckowiak, D.; Majchrzak, M. 
Marciniak, B.; Marciniec, B. New Arylene-Germylene-Vinylene Compounds: Stereoselective Synthesis, Characterization, and Photophysical Properties. Organometallics 2016, 35, 2454-2461.

[11] Iinuma, M.; Moriyama, K.; Togo, H. Various Oxidative Reactions with Novel IonSupported (Diacetoxyiodo) Benzenes. Tetrahedron 2013, 69, 2961-2970.

[12] Baccolini, G.; Boga, C.; Mazzacurati, M. Highly Atom-Economic One-Pot Formation of Three Different C-P Bonds: General Synthesis of Acyclic Tertiary Phosphine Sulfides. J. Org. Chem. 2005, 70, 4774-4777.

[13] Murov, S. L., Handbook of Photochemistry, Marcel Dekker, New York, 1973.

[14] For initiating the thio-ene reaction by UV-light or heat: a) Brummelhuis, N. T.; Diehl, C.; Schlaad, H. Thiol-Ene Modification of 1, 2-Polybutadiene Using UV Light or Sunlight. Macromolecules 2008, 41, 9946-9947. b) Tyson, E. L.; Ament, M. S.; Yoon, T. P. Transition Metal Photoredox Catalysis of Radical Thiol-Ene Reactions. J. Org. Chem. 2013, 78, 2046-2050; c) Rissing, C.; Son, D. Y. ThiolEne Reaction for the Synthesis of Multifunctional Branched Organosilanes. Organometallics 2008, 27, 5394-5397.

[15] Kim, H.; Lee, C. Visible-Light-Induced Photocatalytic Reductive Transformations of Organohalides. Angew. Chem. Int. Ed. 2012, 51, 12303-12306.

[16] a) Nicewicz, D. A.; MacMillan, D.W. C. Merging Photoredox Catalysis with Organocatalysis: the Direct Asymmetric Alkylation of Aldehydes. Science 2008, 322, 77-80; b) Shih, H.W.; VanderWal, M. N.; Grange, R. L.; MacMillan, D. W. C. Enantioselective $\alpha$-Benzylation of Aldehydes via Photoredox Organocatalysis. J. Am. Chem. Soc. 2010, 132, 13600-13603.

[17] Revol, G.; McCallum, T.; Morin, M.; Gagosz, F.; Barriault, L. Photoredox Transformations with Dimeric Gold Complexes. Angew. Chem. Int. Ed. 2013, 52, 13342-13345. 


\section{NMR spectra}
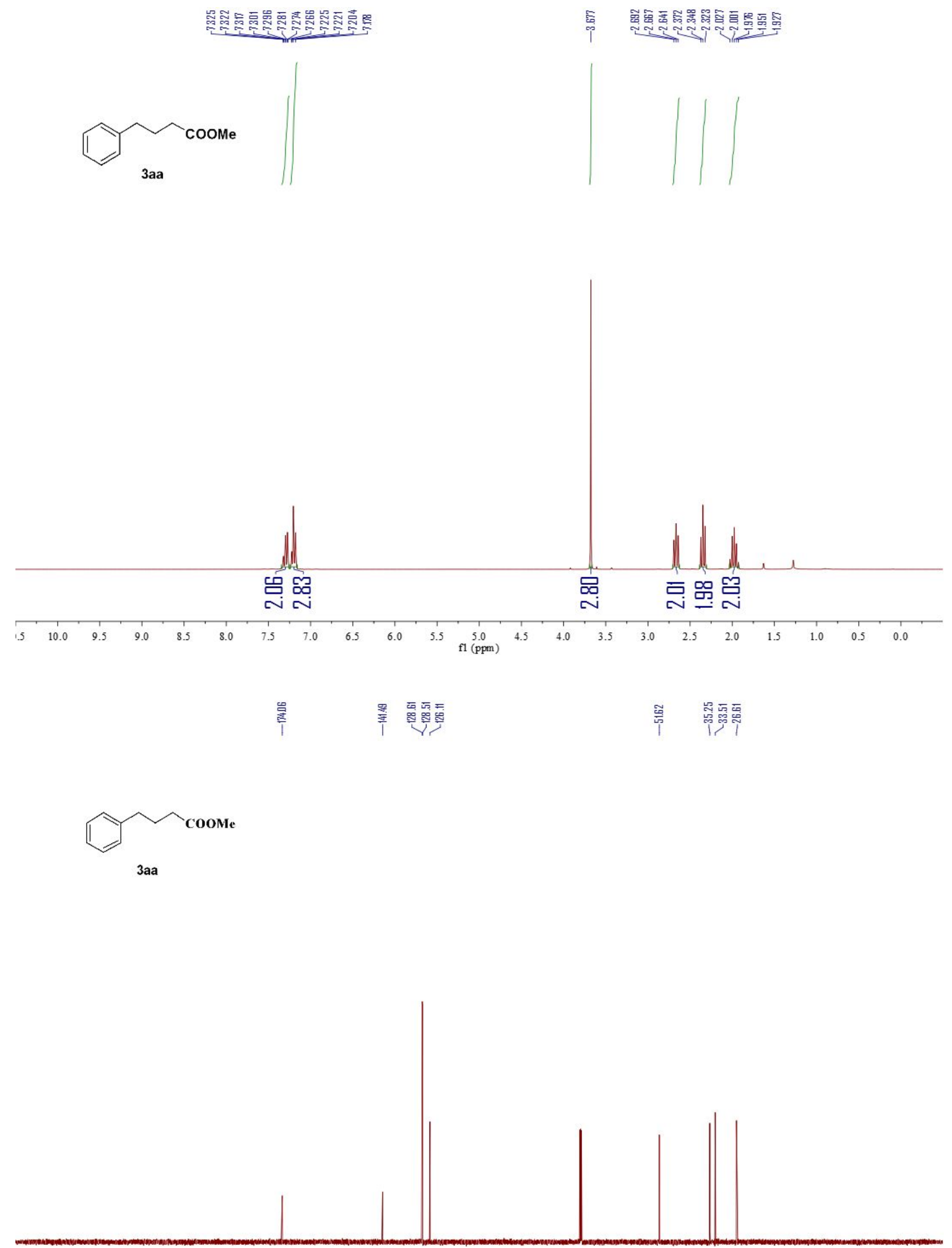

$\begin{array}{lllllllllllllllllllllllllllllllllllllllllllllllllll}60 & 250 & 240 & 230 & 220 & 210 & 200 & 190 & 180 & 170 & 160 & 150 & 140 & 130 & 120 & 110 & 100 & 90 & 80 & 70 & 60 & 50 & 40 & 30 & 20 & 10 & 0 & -10 & -20 & -30 & -4\end{array}$ 

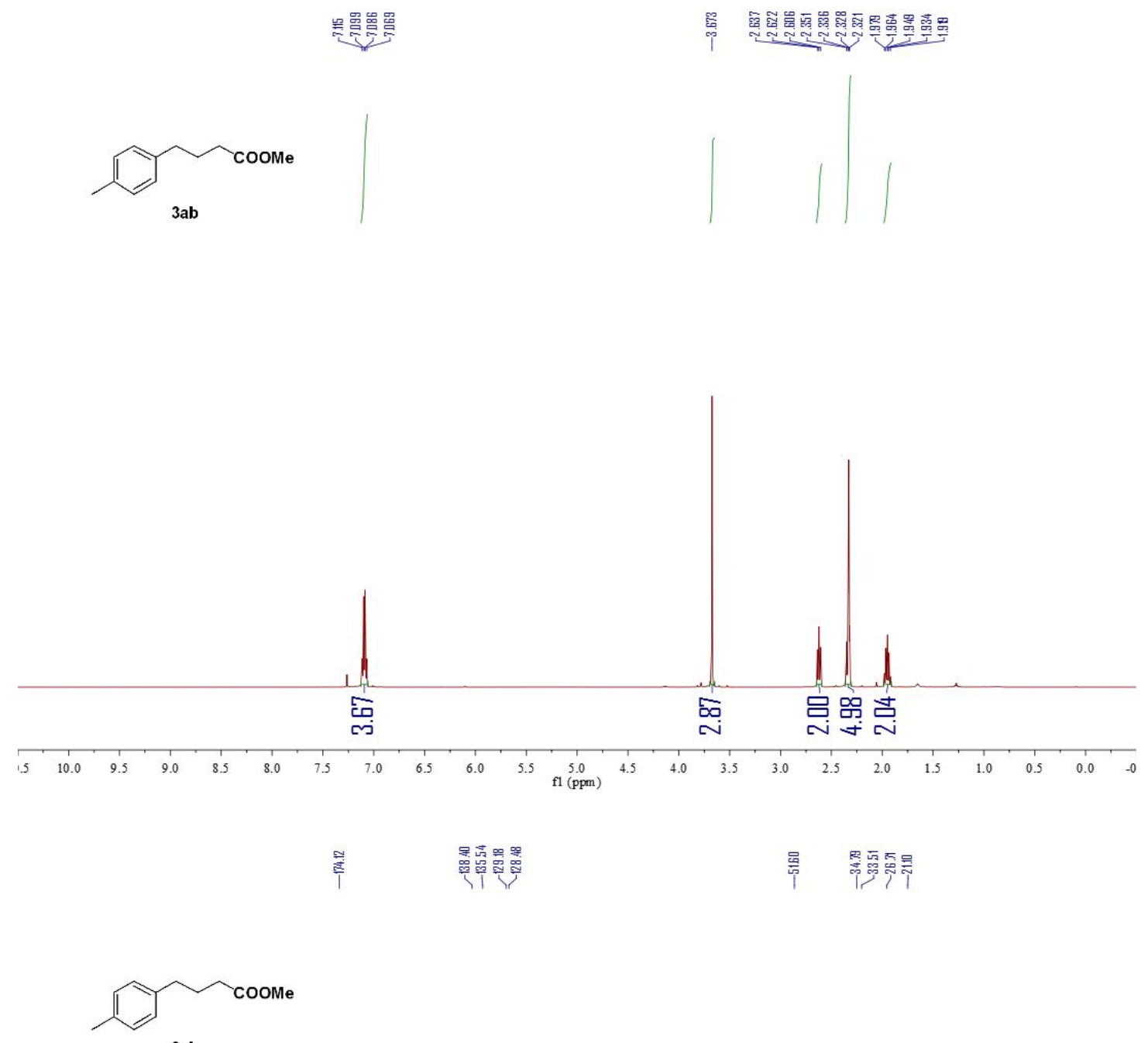

$3 a b$

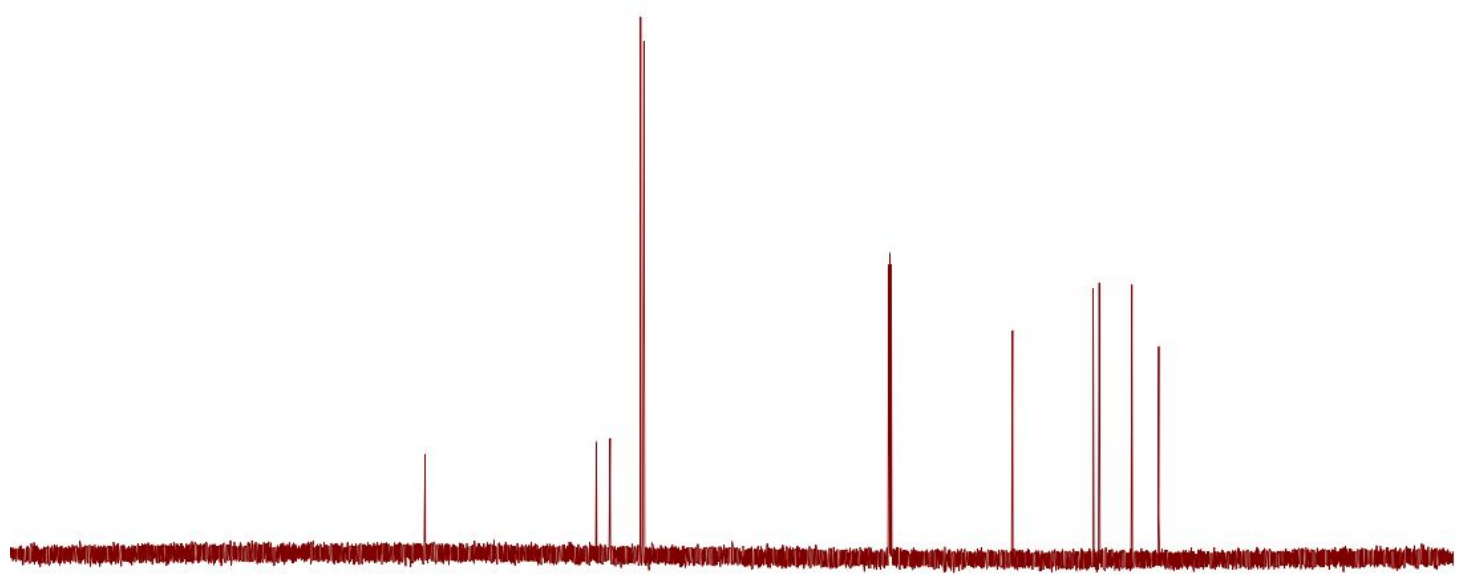

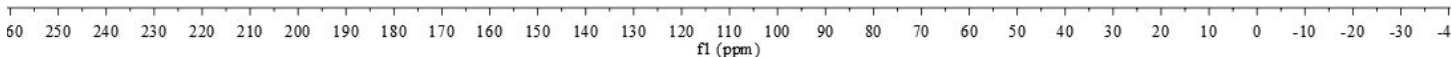



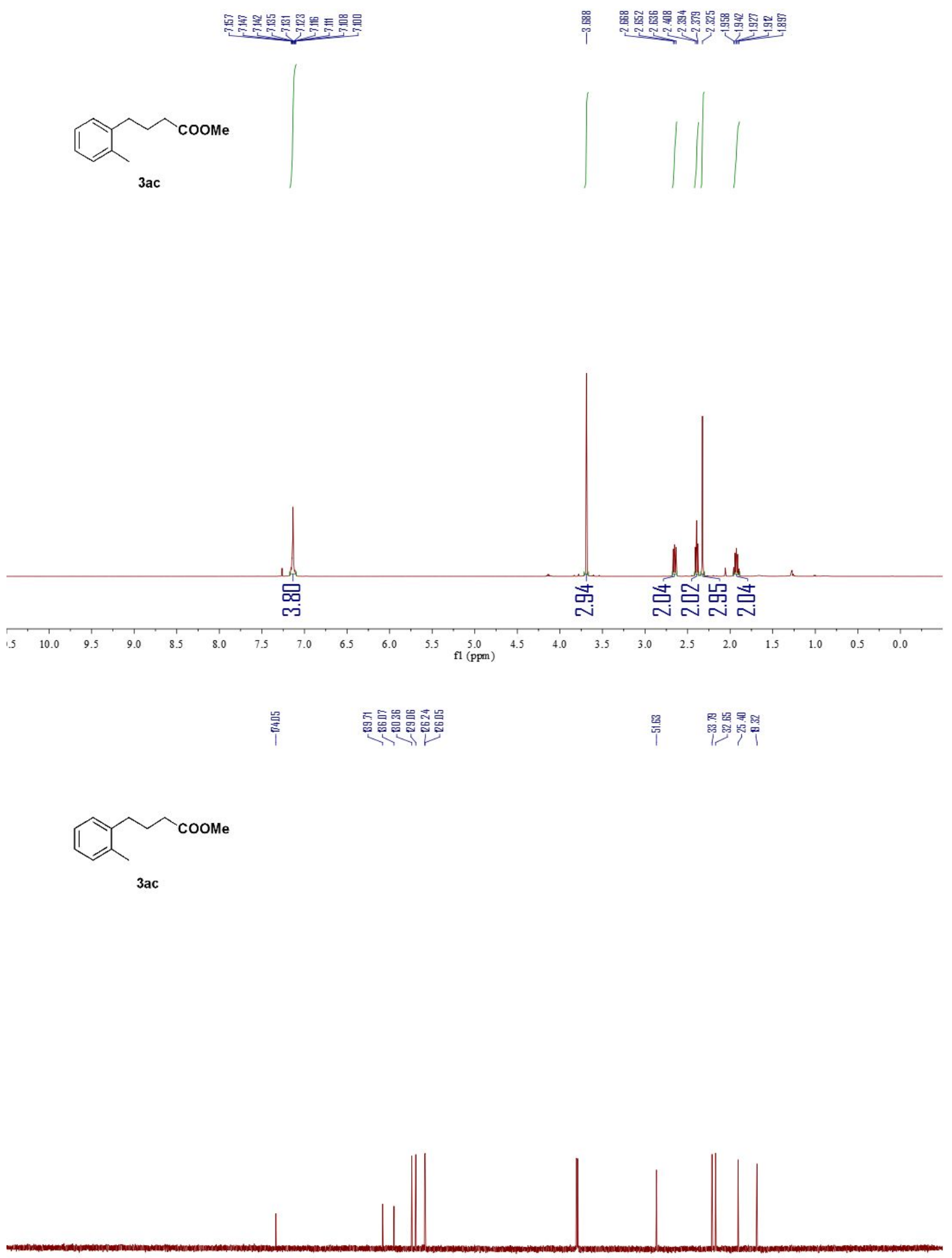

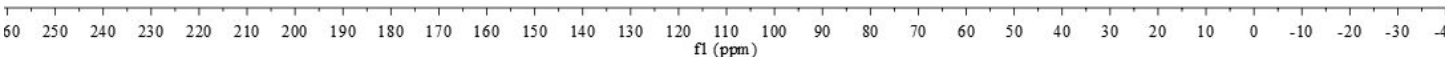


<smiles>COCCCc1c(C)cc(C)cc1C</smiles>

3ad
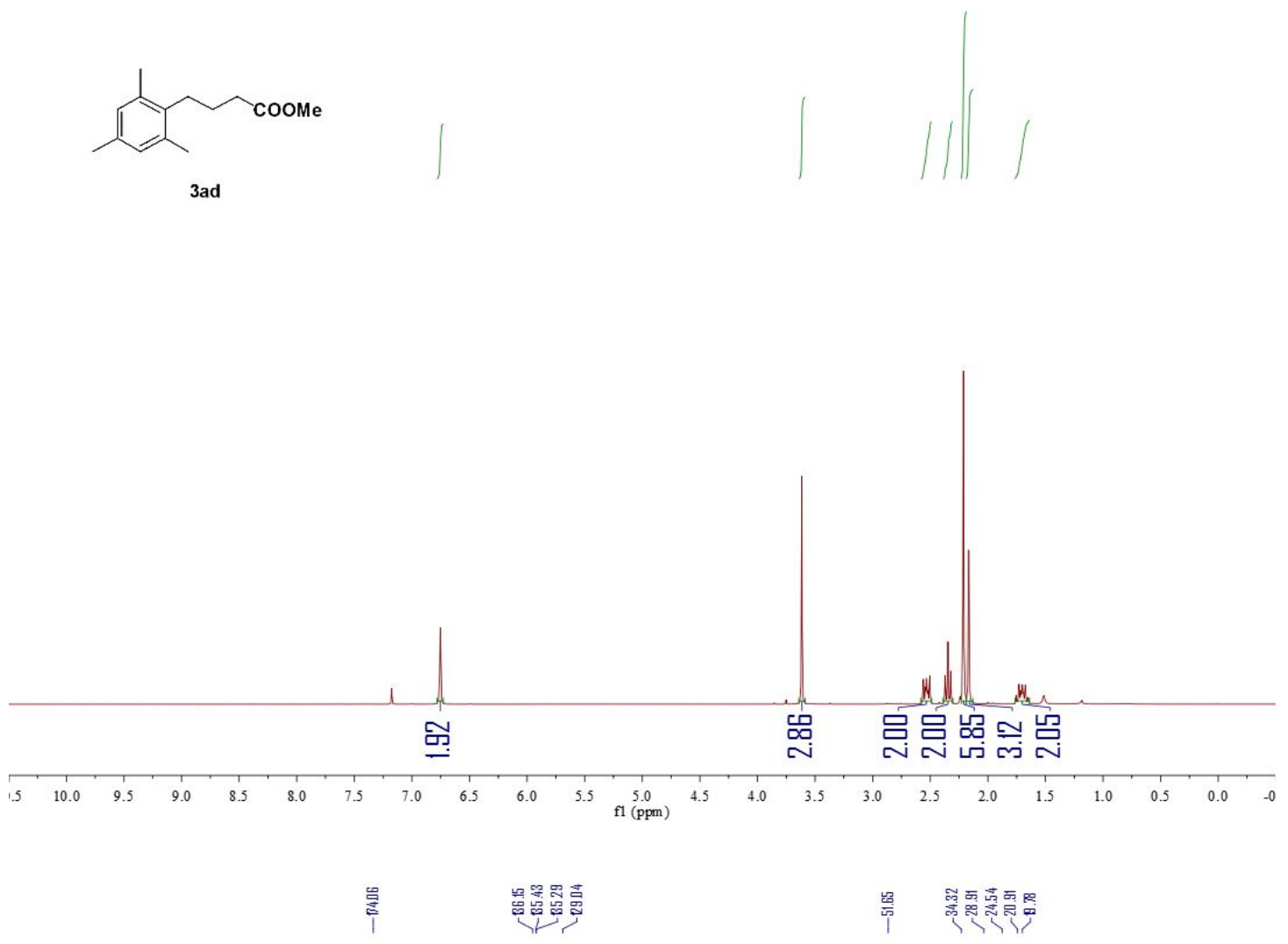<smiles>CC(=O)CCCc1c(C)cc(C)cc1C</smiles>

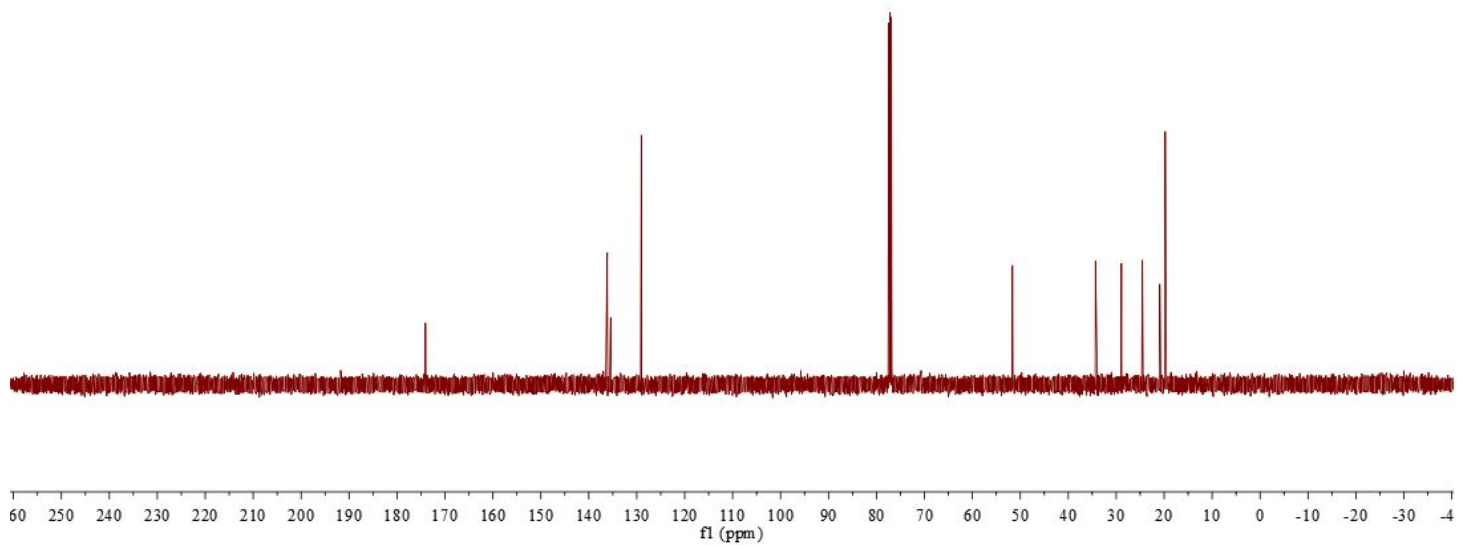



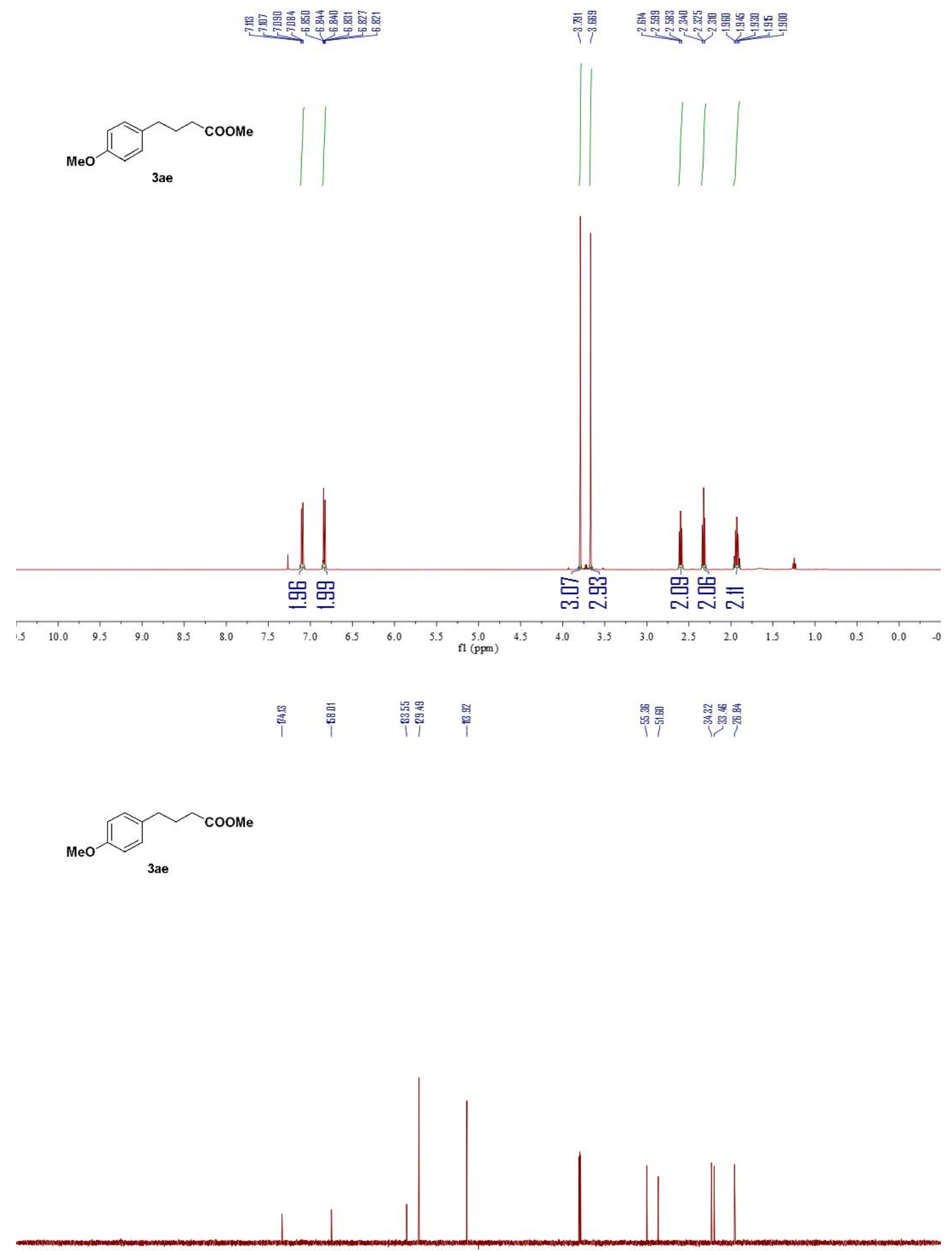

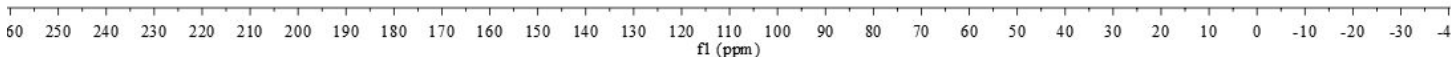



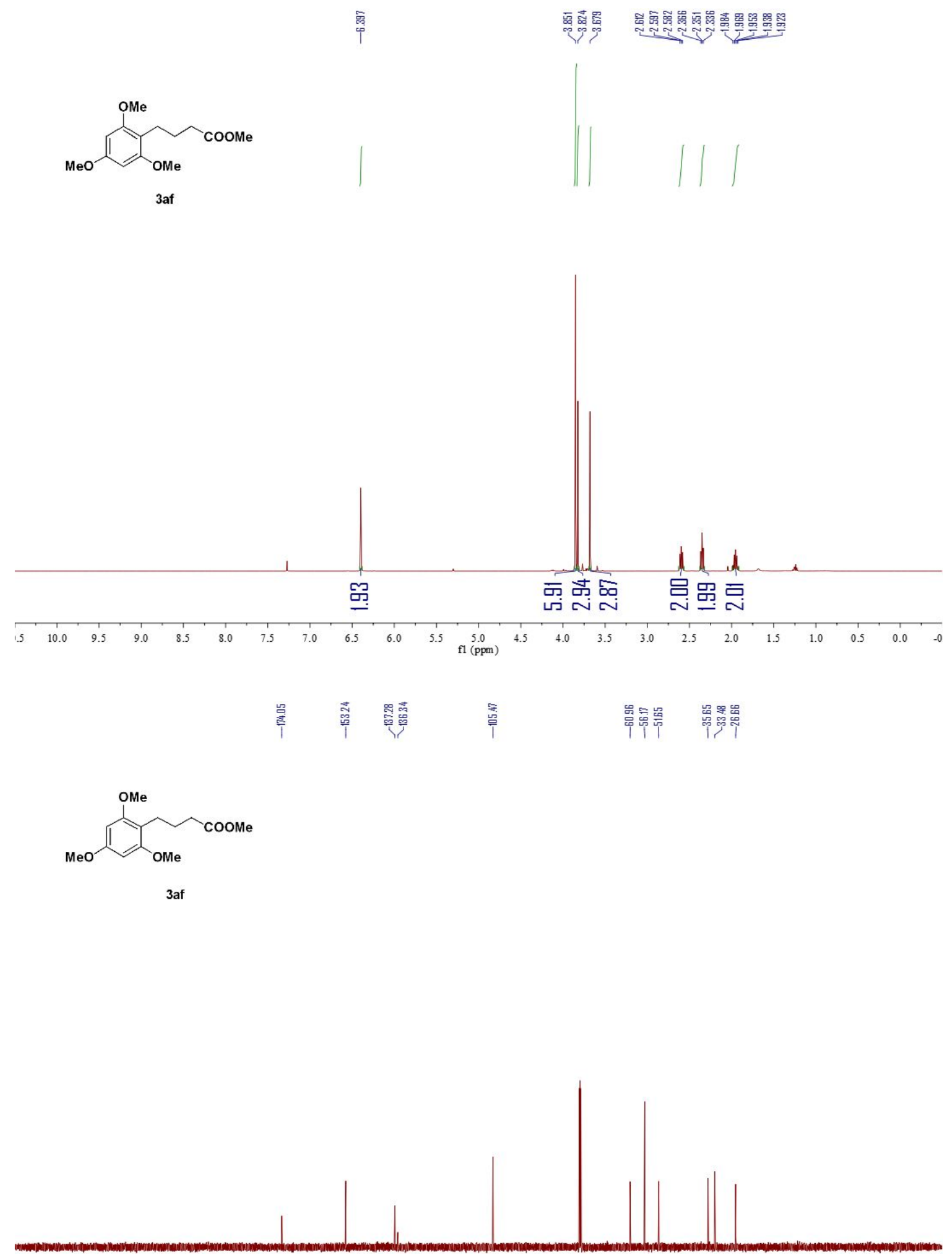

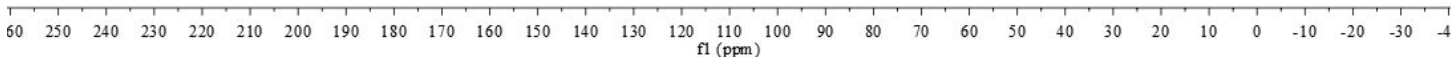



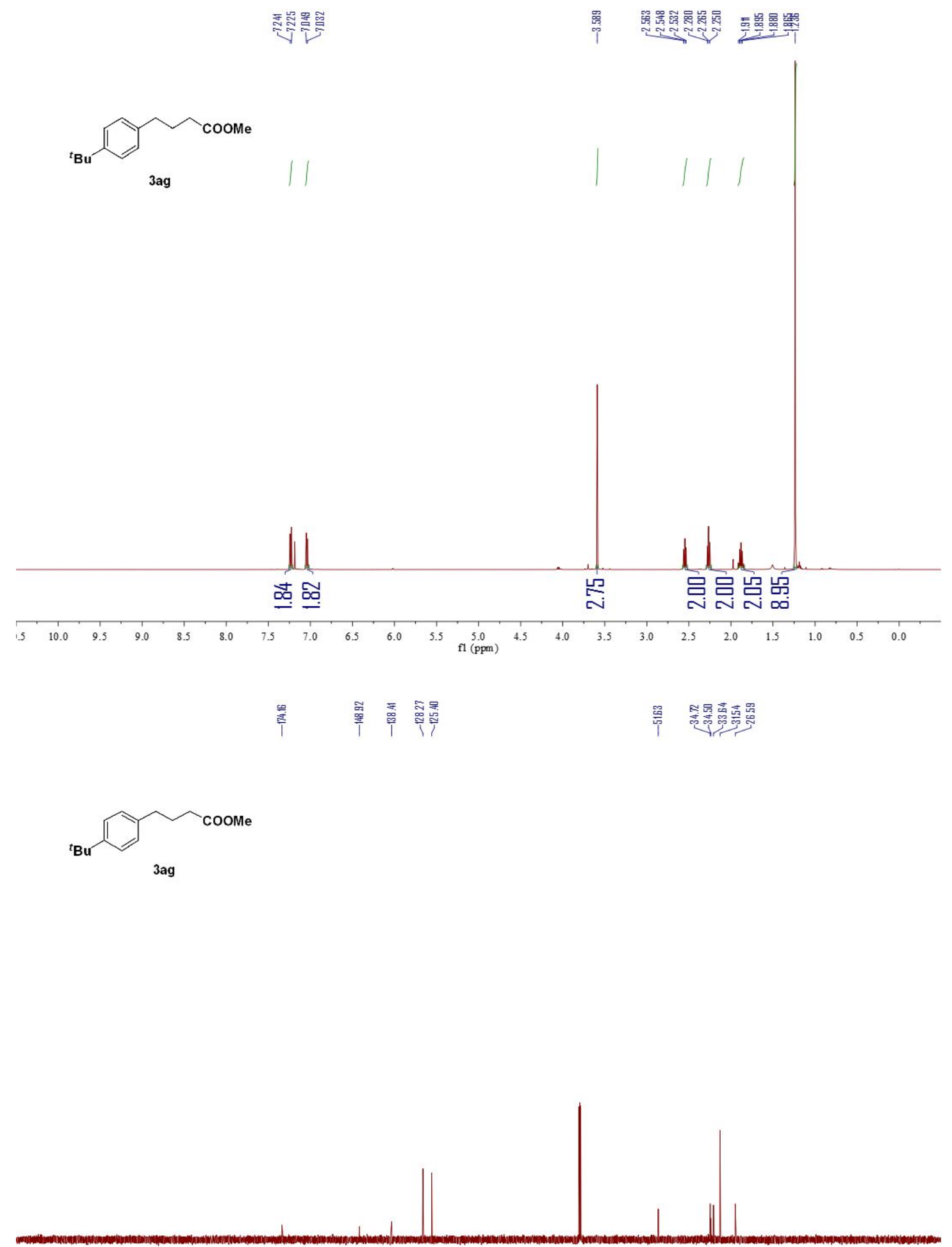

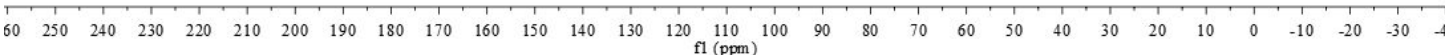




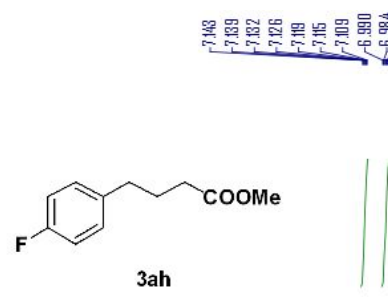

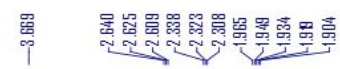
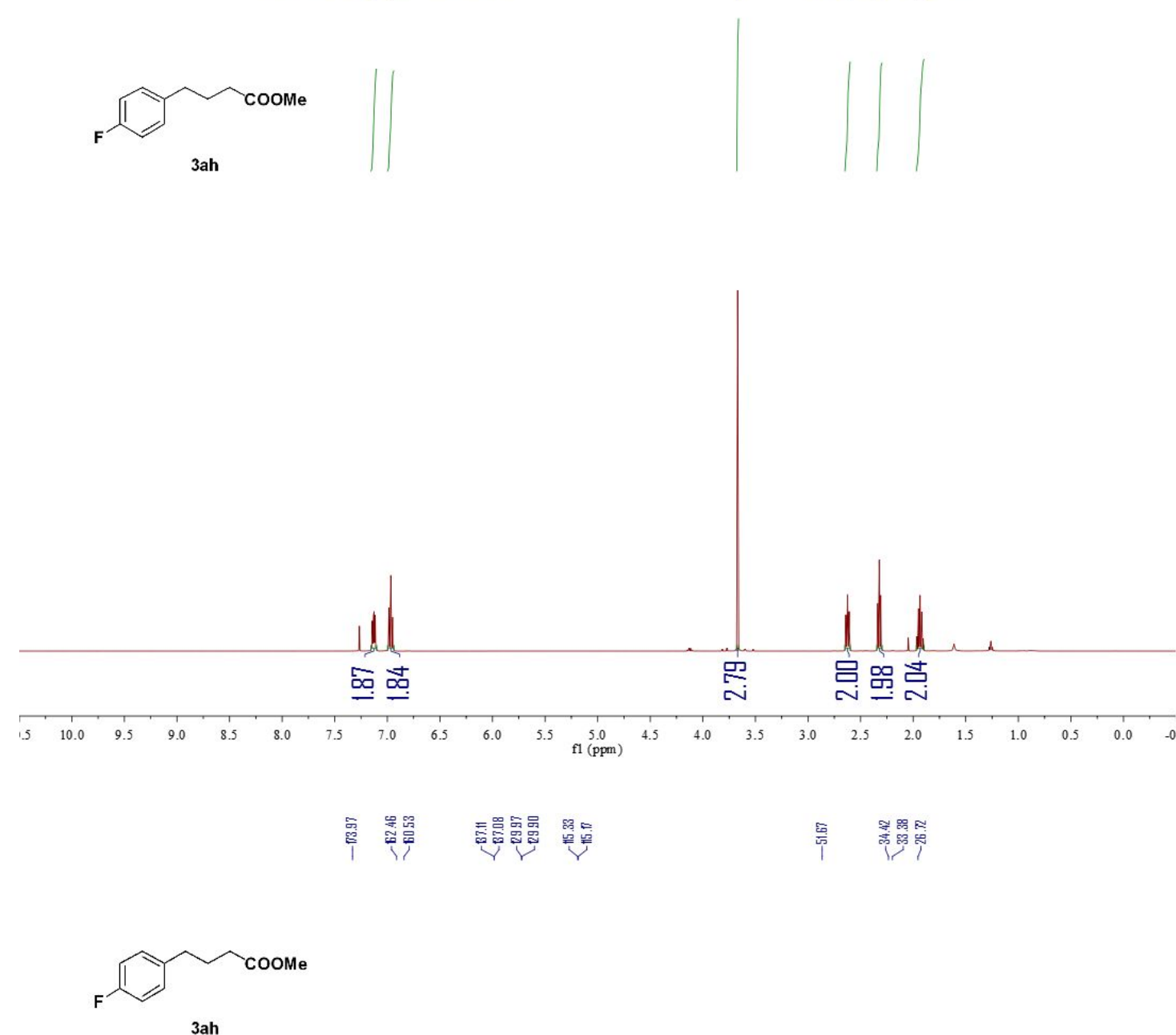

3ah

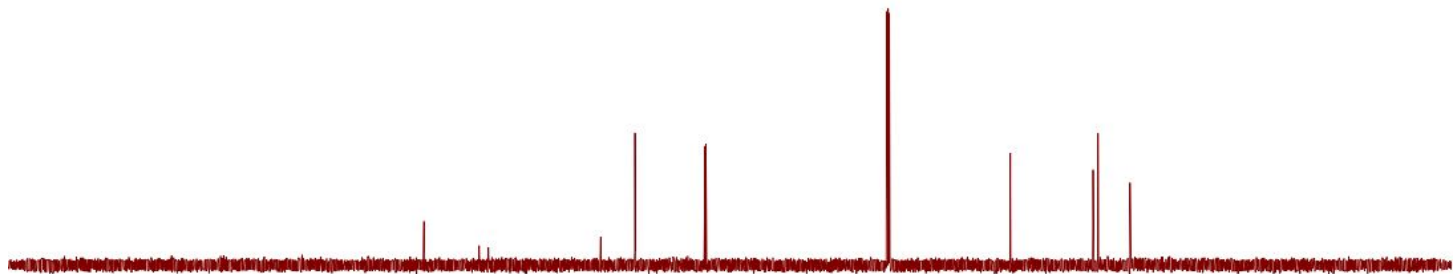

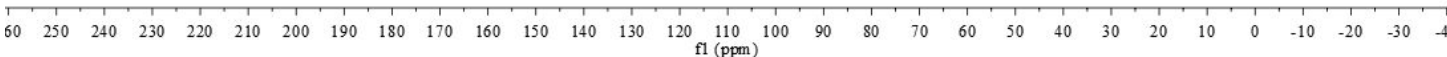




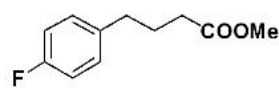

3ah

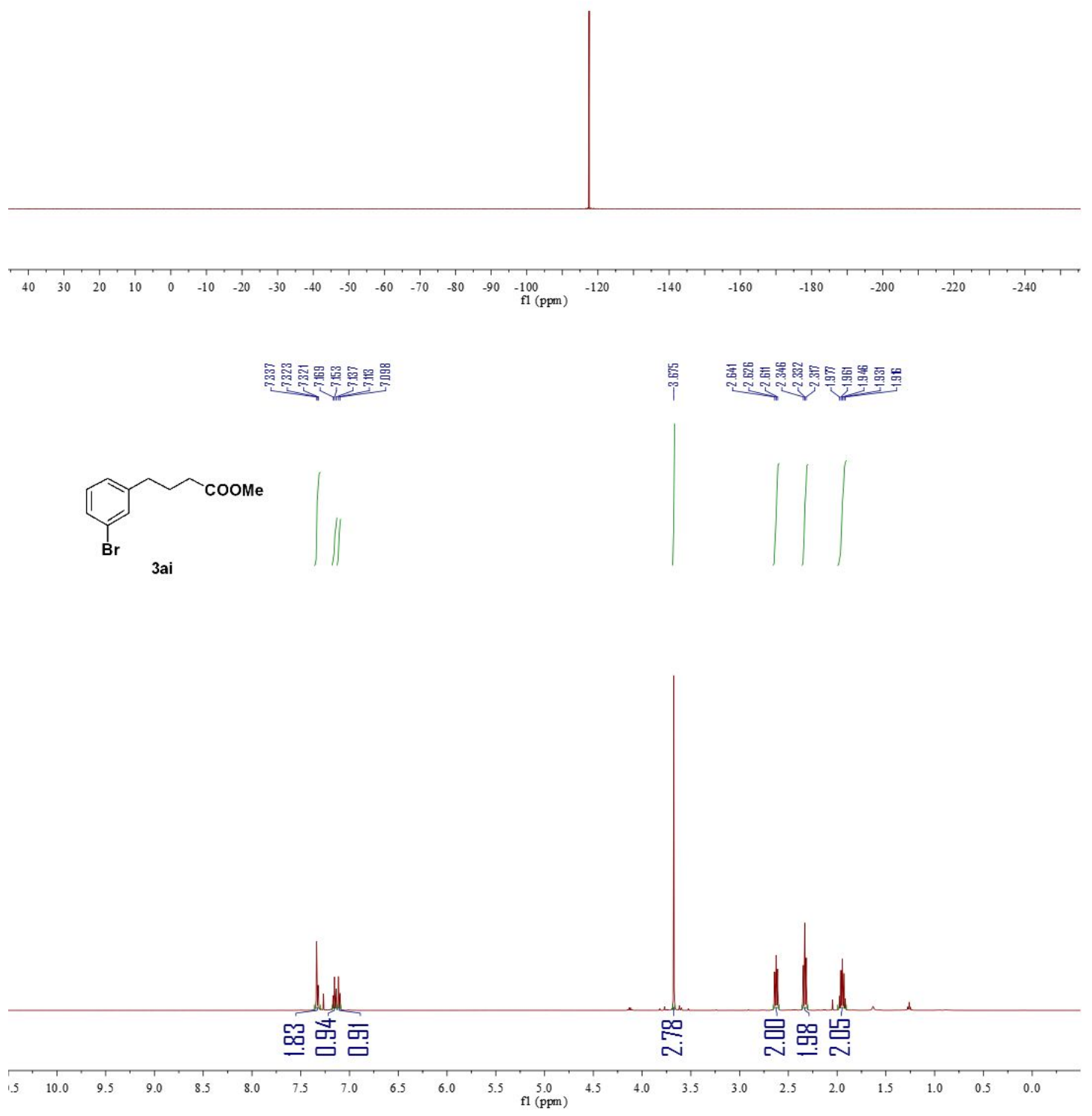




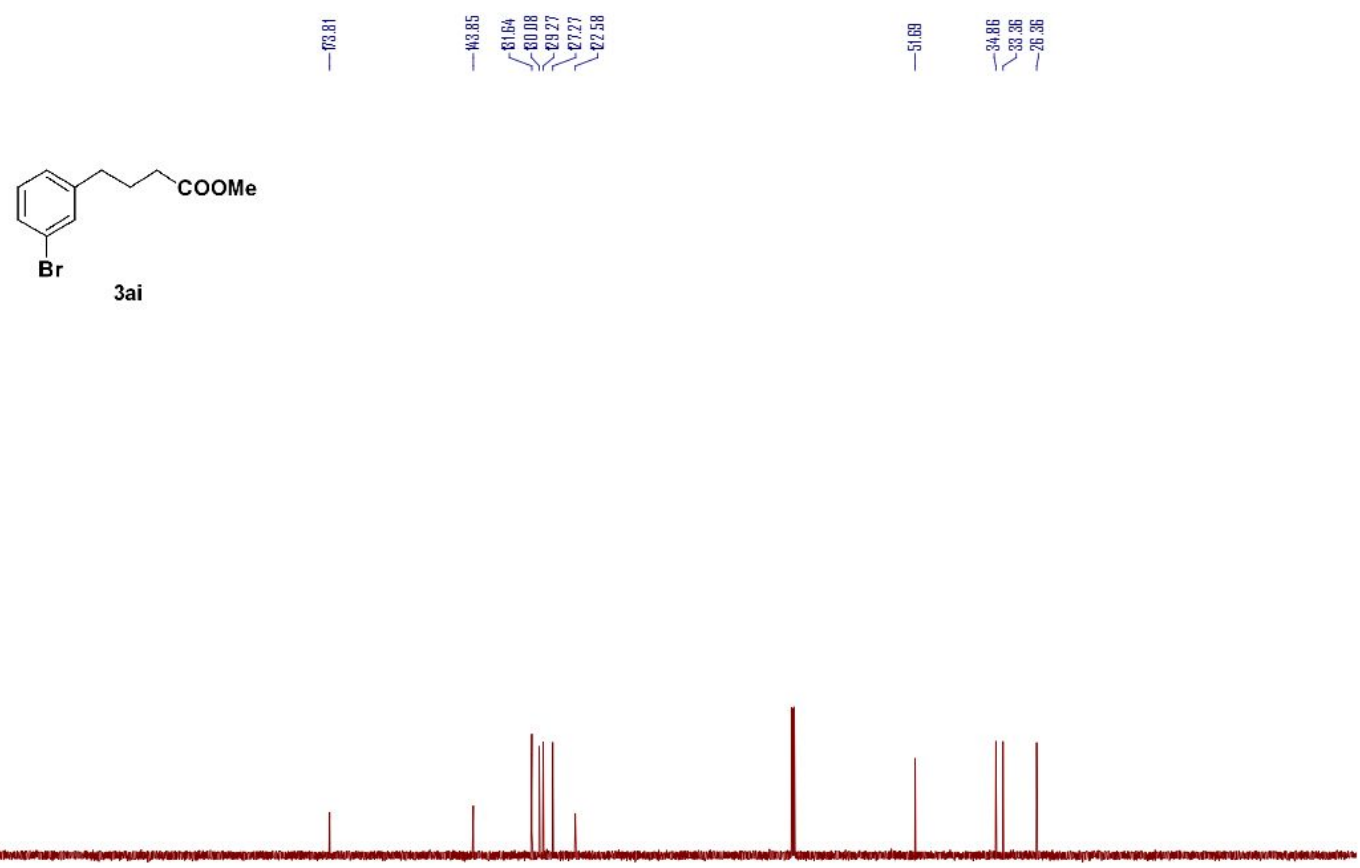

$\begin{array}{lllllllllllllllllllllllllllllllllll}60 & 250 & 240 & 230 & 220 & 210 & 200 & 190 & 180 & 170 & 160 & 150 & 140 & 130 & 120 & 110 & 100 & 90 & 80 & 70 & 60 & 50 & 40 & 30 & 20 & 10 & 0 & -10 & -20 & -30 & -4\end{array}$
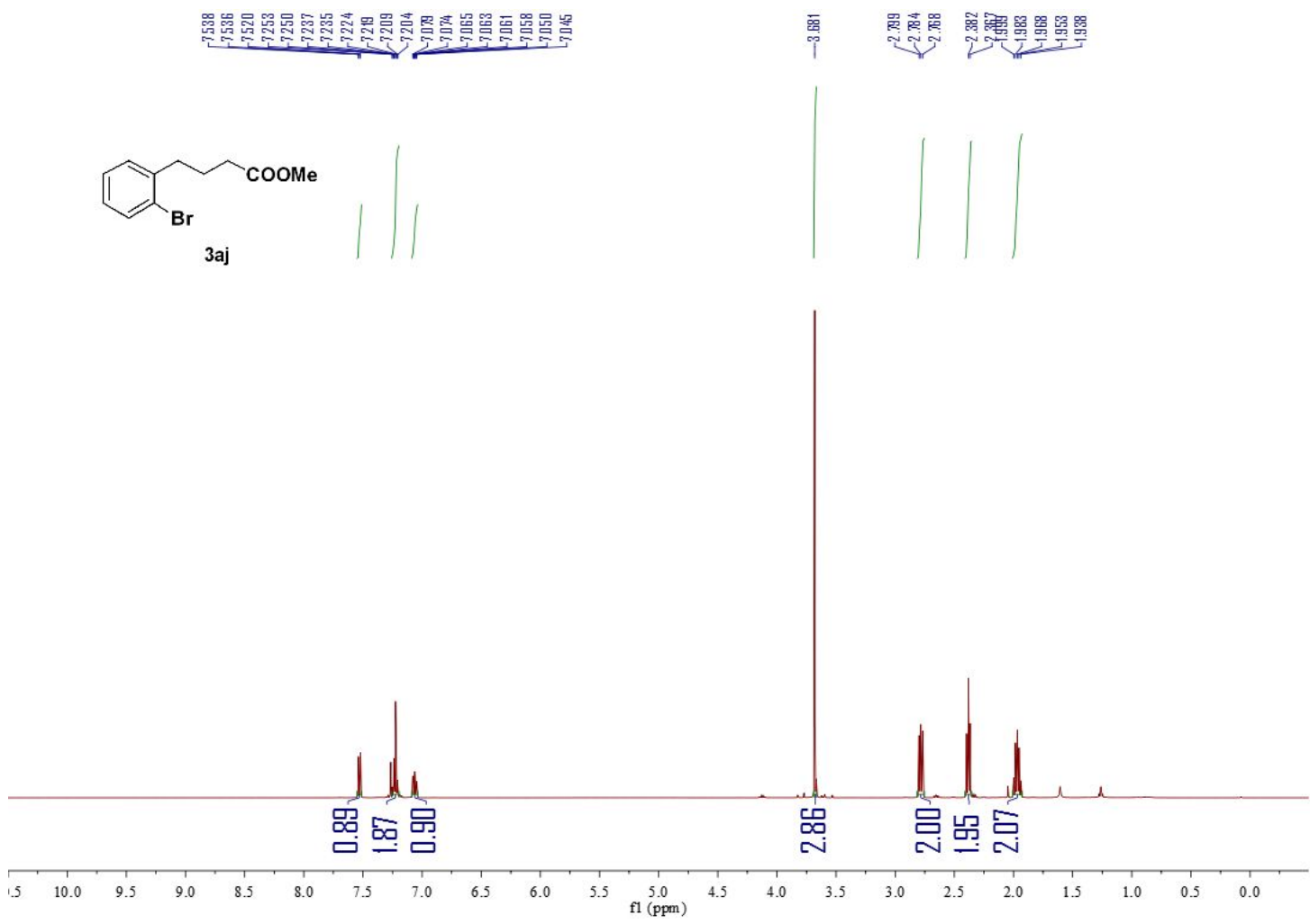


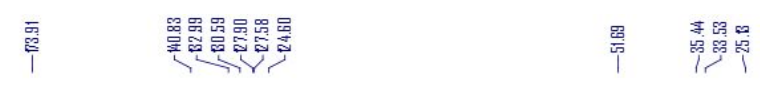

$\overbrace{\mathrm{Br}}^{\text {coome }}$

3aj

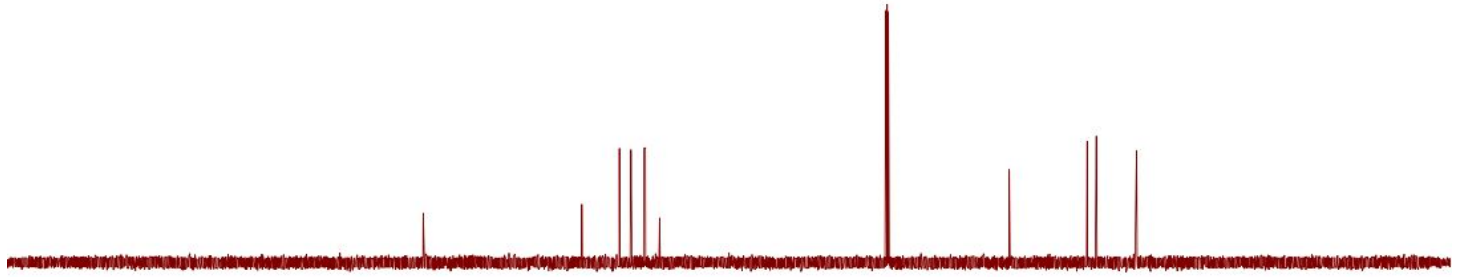

$\begin{array}{lllllllllllllllllllllllllllllllll}60 & 250 & 240 & 230 & 220 & 210 & 200 & 190 & 180 & 170 & 160 & 150 & 140 & 130 & 120 & 110 & 100 & 90 & 80 & 70 & 60 & 50 & 40 & 30 & 20 & 10 & 0 & -10 & -20 & -30 & -4\end{array}$

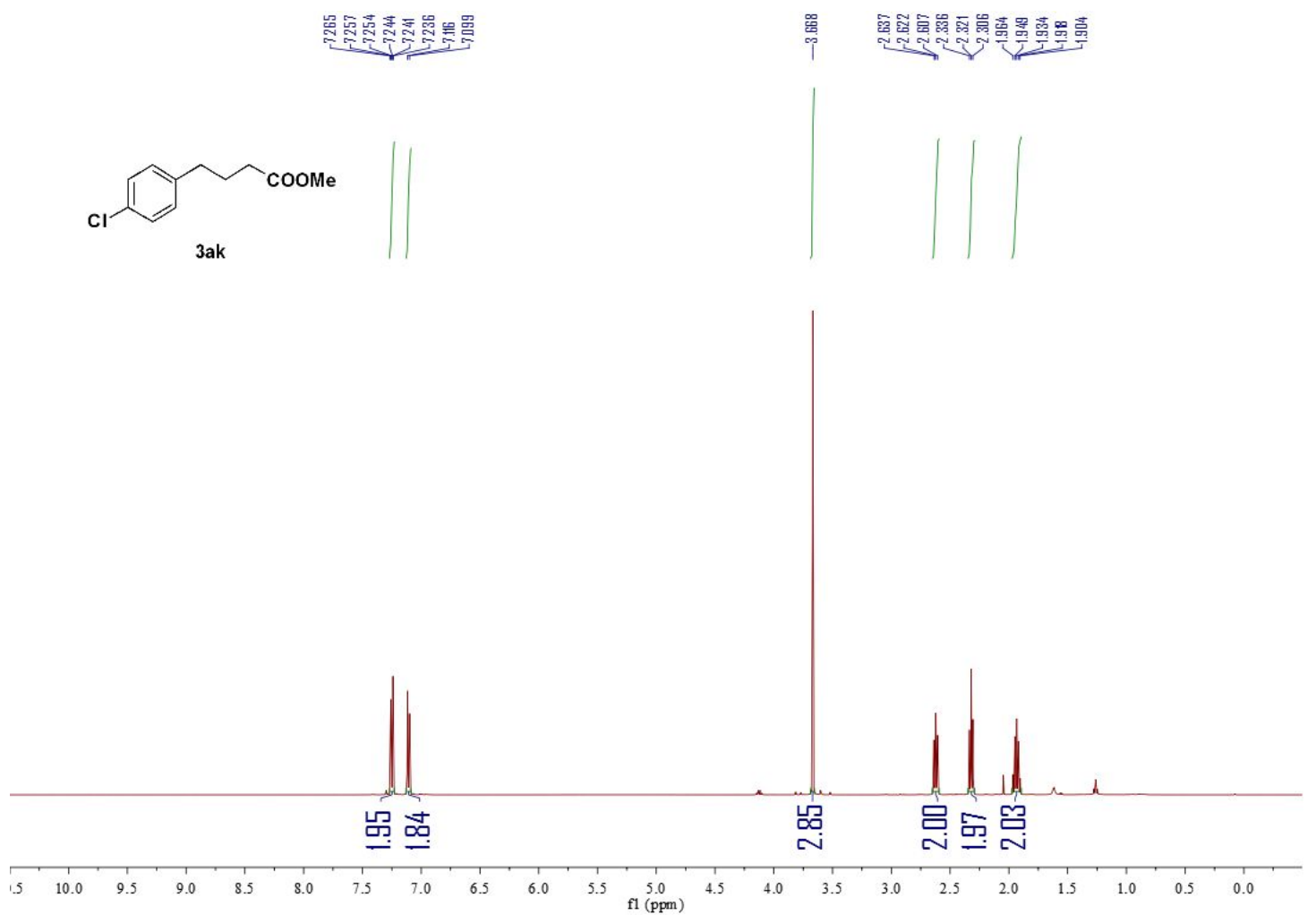




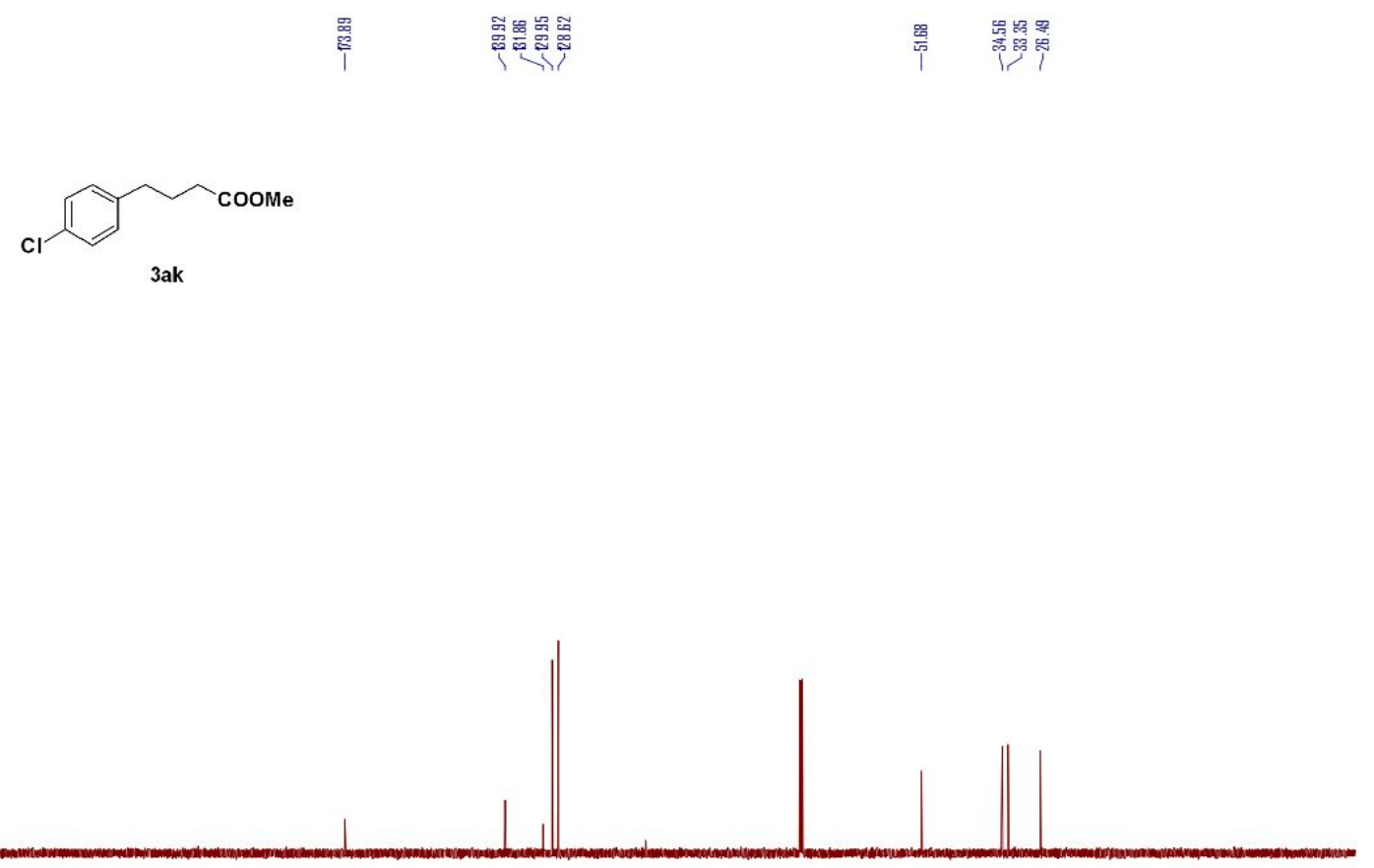

$\begin{array}{lllllllllllllllllllllllllllllllllllll}60 & 250 & 240 & 230 & 220 & 210 & 200 & 190 & 180 & 170 & 160 & 150 & 140 & 130 & 120 & 110 & 100 & 90 & 80 & 70 & 60 & 50 & 40 & 30 & 20 & 10 & 0 & -10 & -20 & -30 & -4\end{array}$
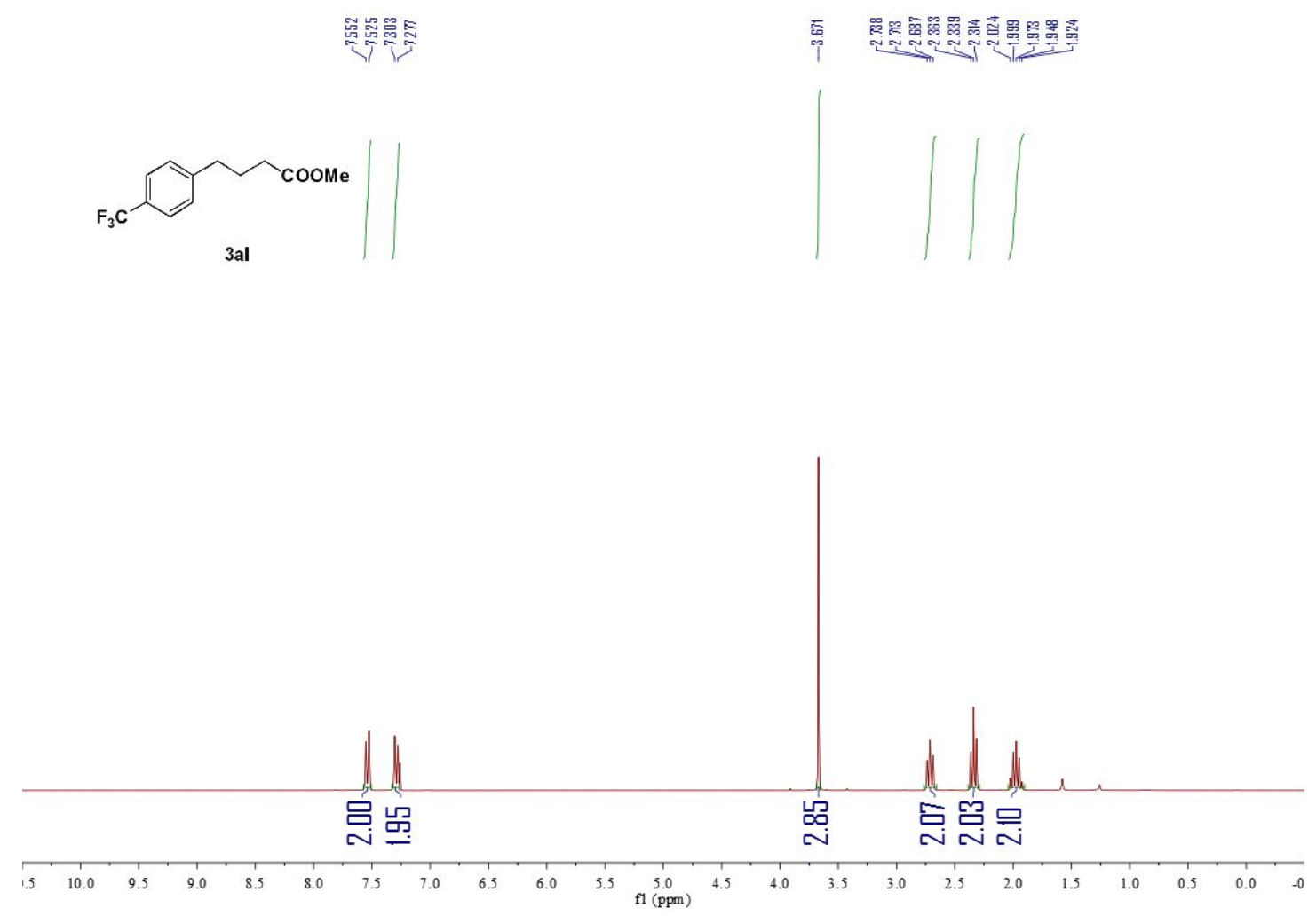


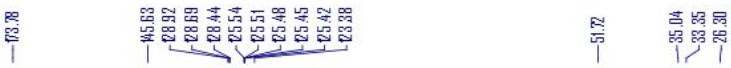

coome

3al

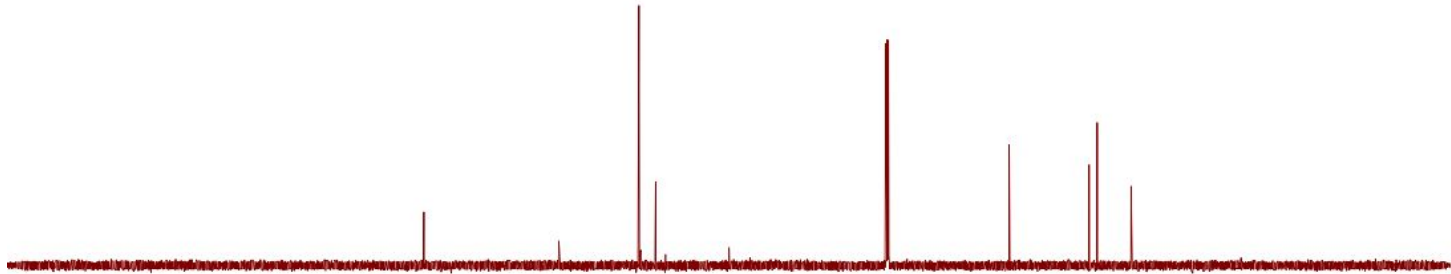

$\begin{array}{lllllllllllllllllllllllllllllllllll}60 & 250 & 240 & 230 & 220 & 210 & 200 & 190 & 180 & 170 & 160 & 150 & 140 & 130 & 120 & 110 & 100 & 90 & 80 & 70 & 60 & 50 & 40 & 30 & 20 & 10 & 0 & -10 & -20 & -30 & -4\end{array}$

彥

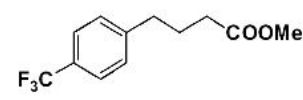

3al

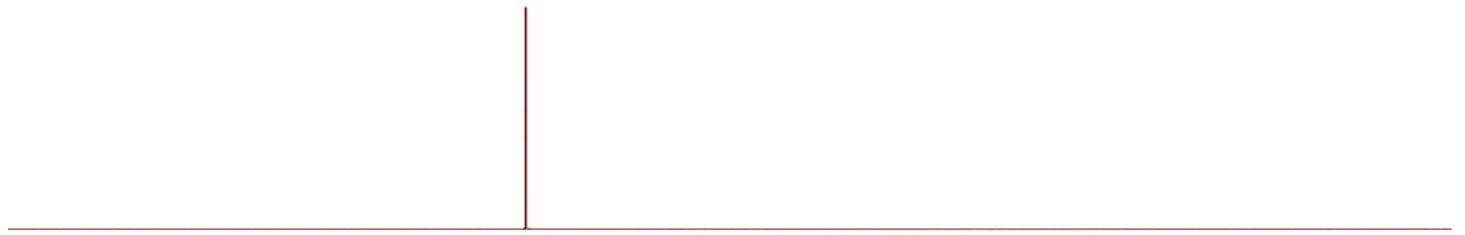

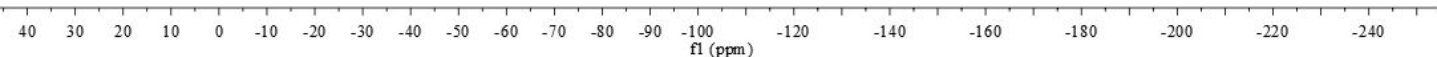




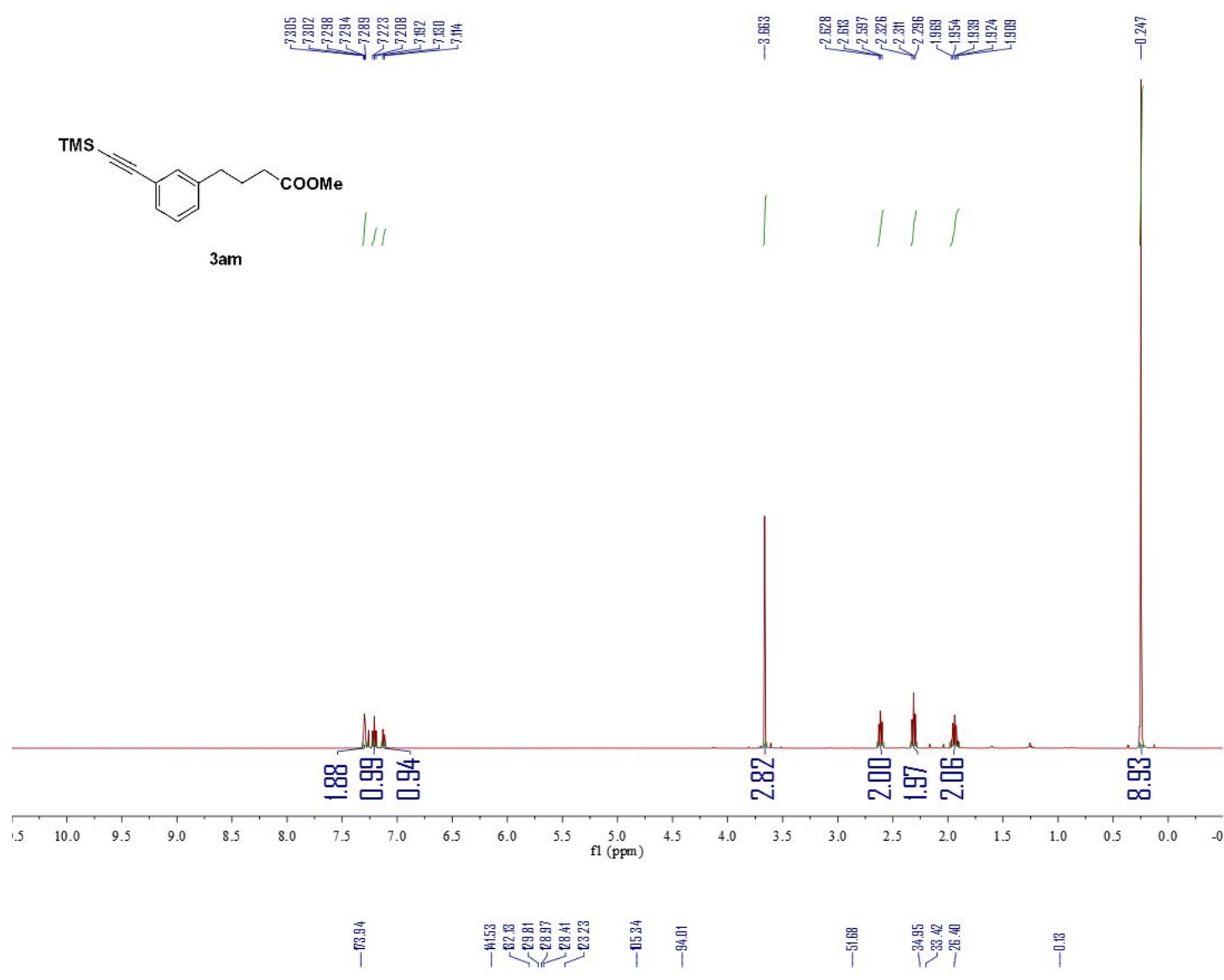

TMS<smiles>C#Cc1cccc(CCCC(C)=O)c1</smiles>

3am

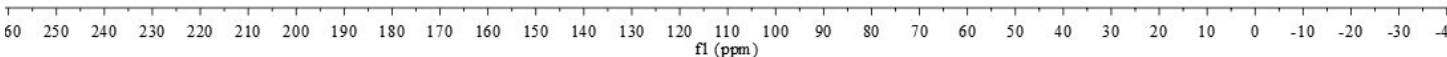



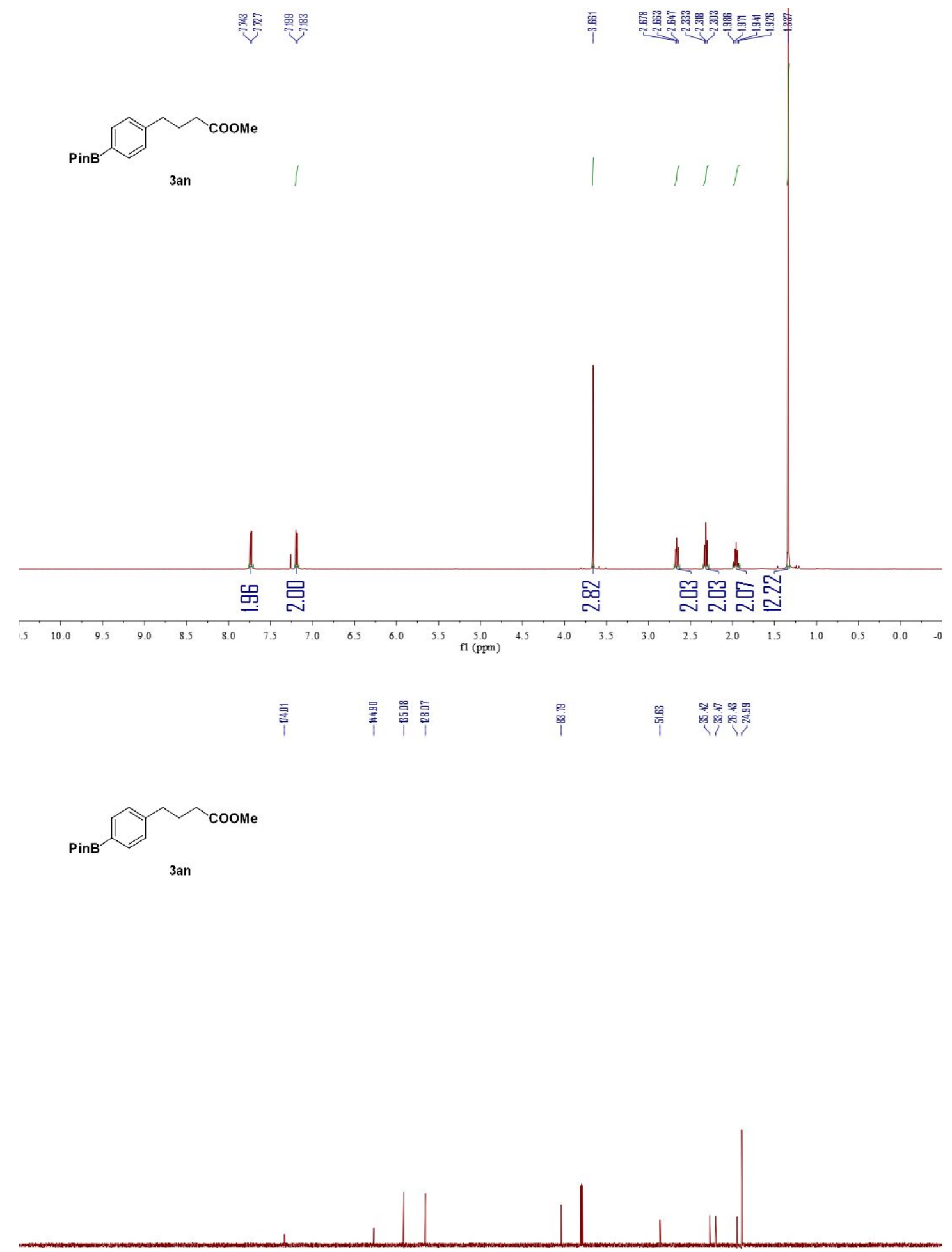

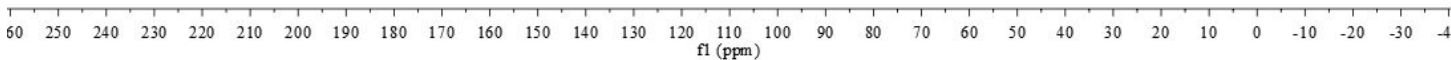




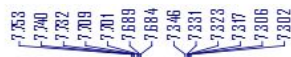

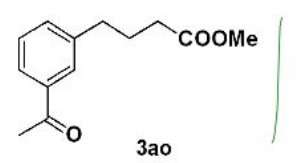

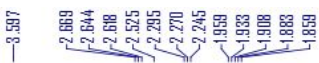

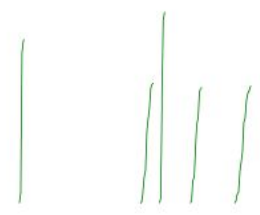

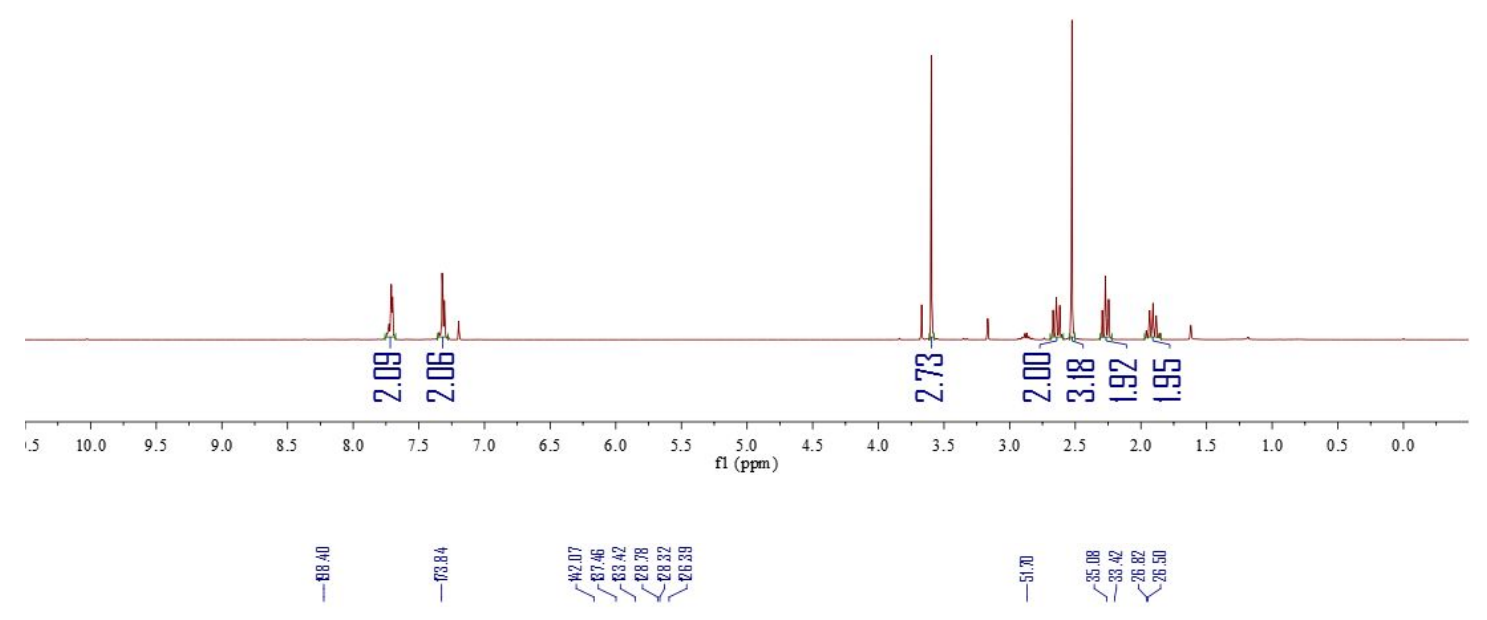
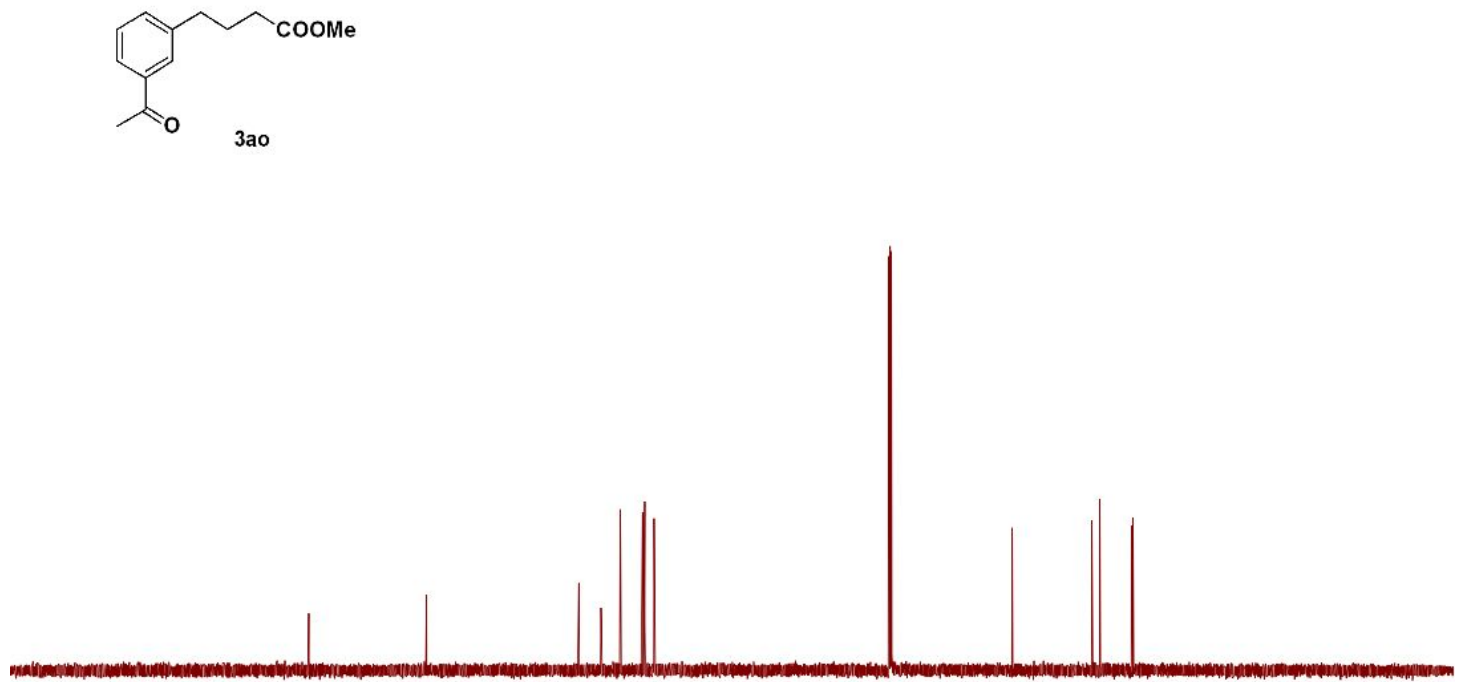

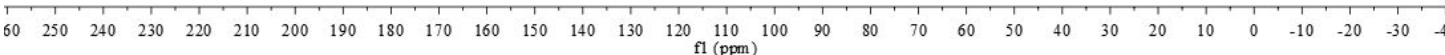



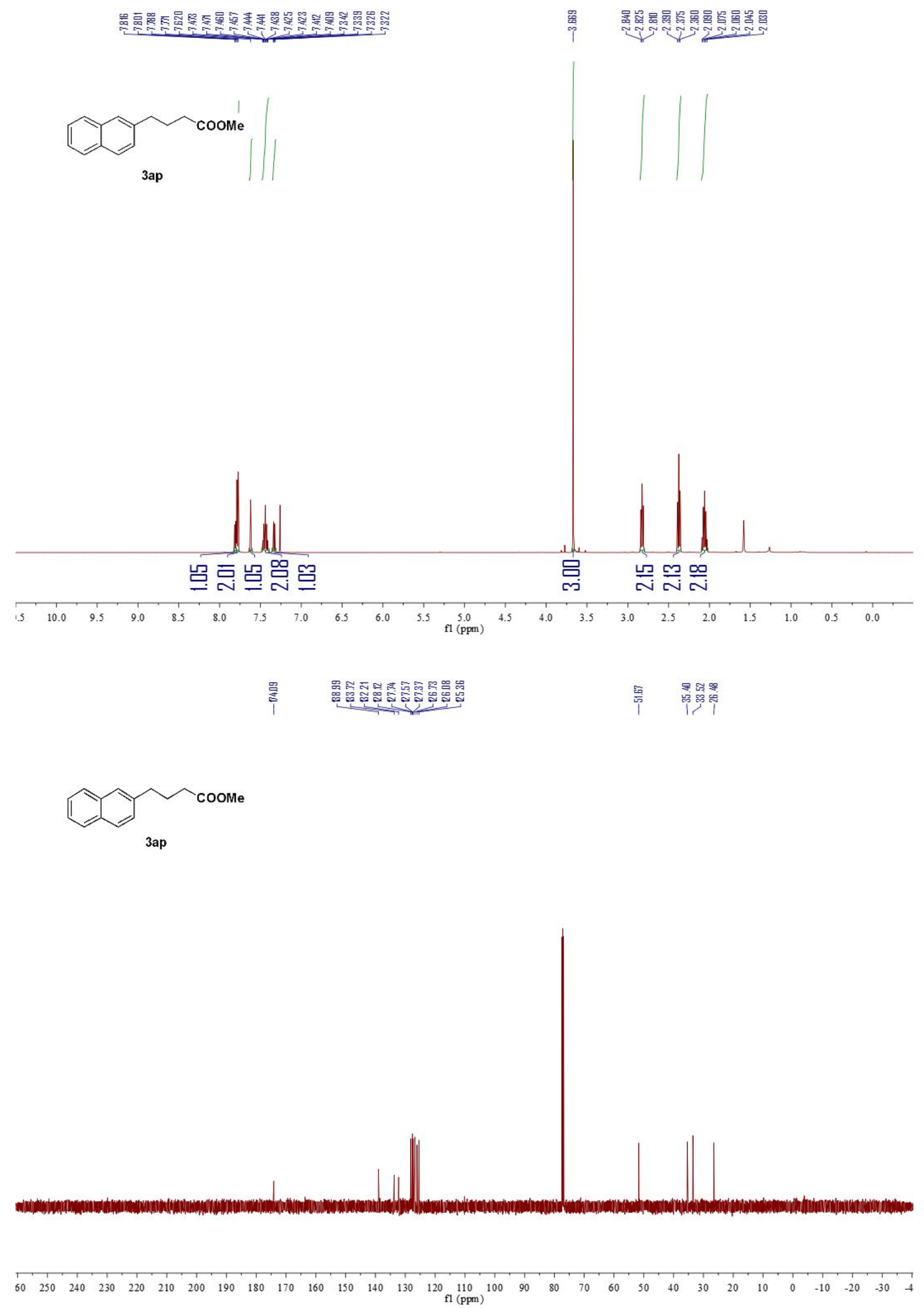

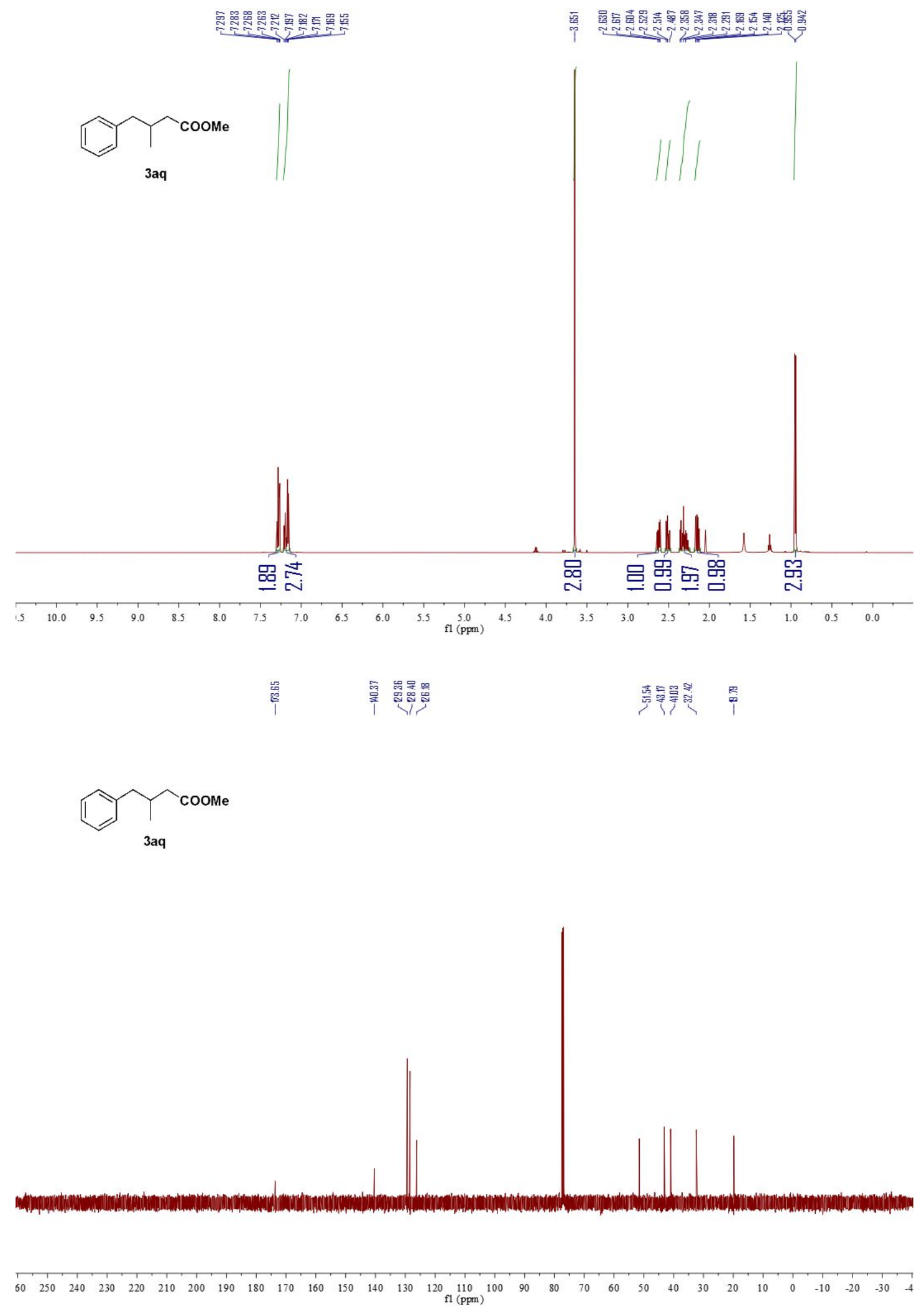

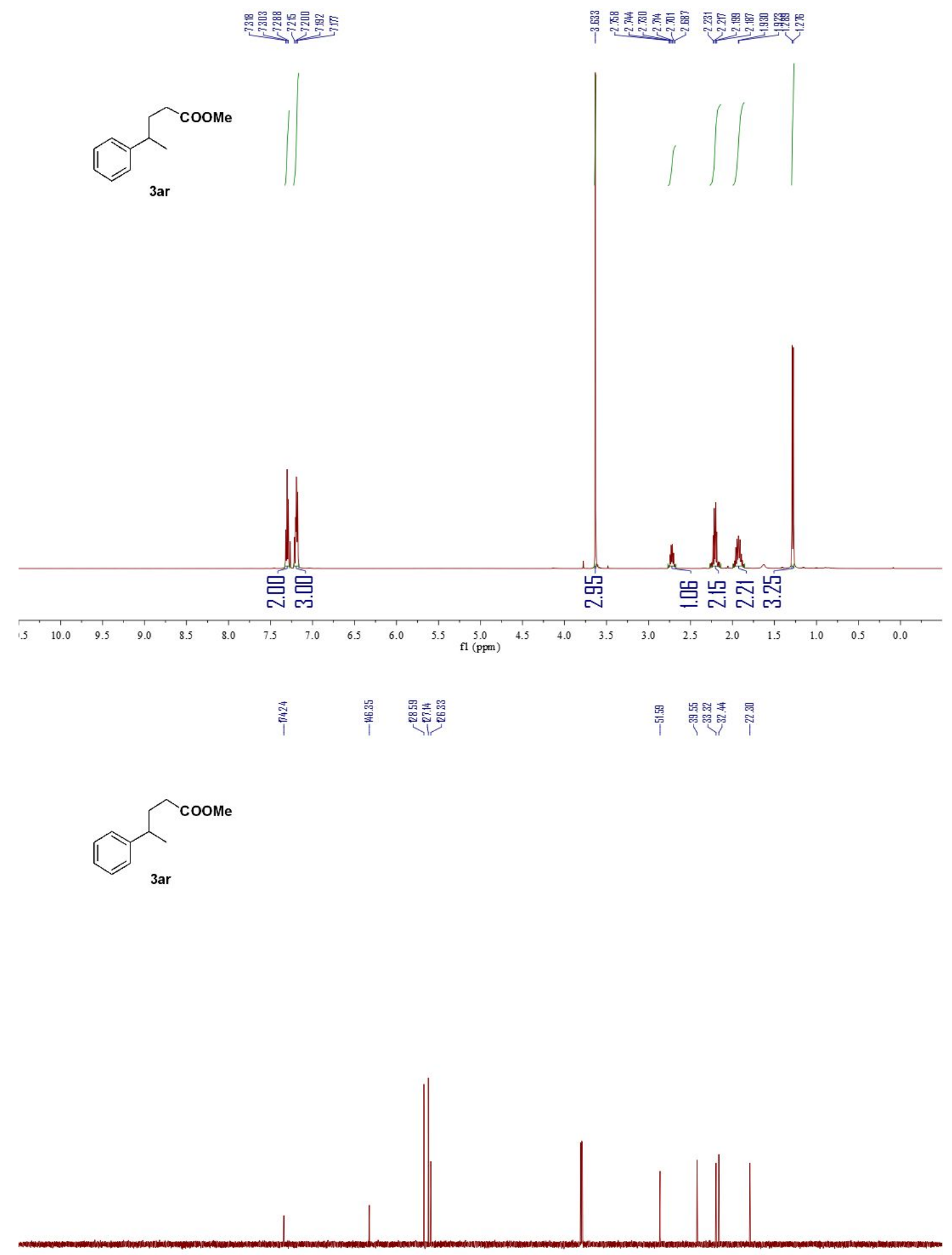

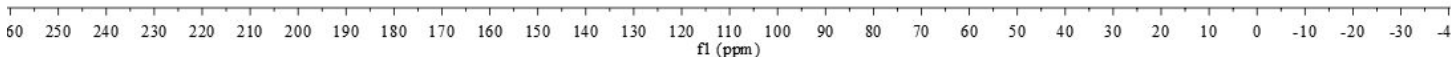



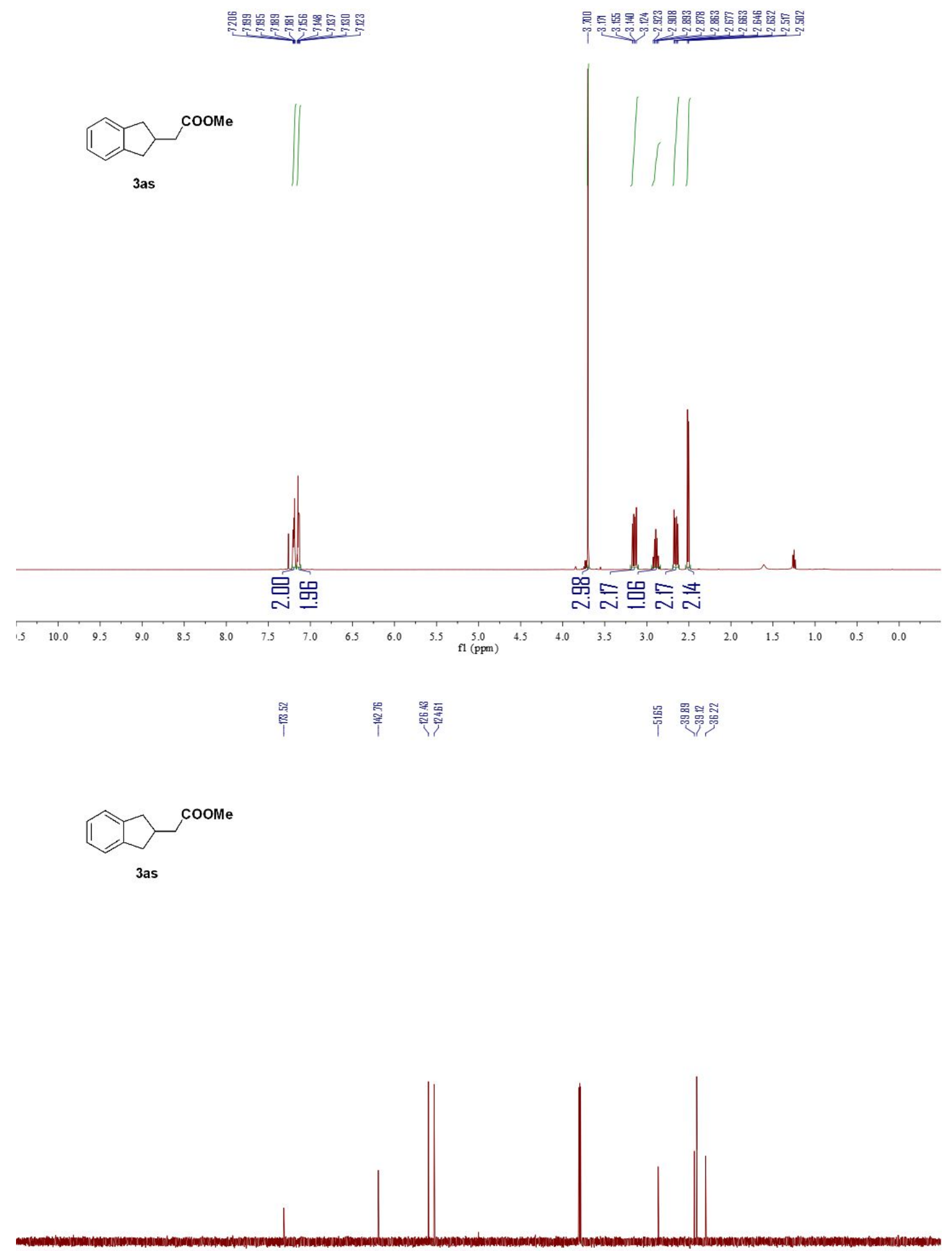

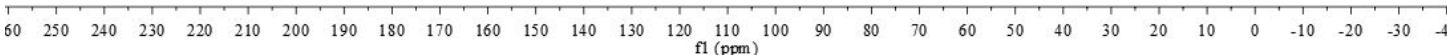




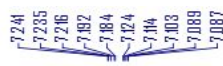

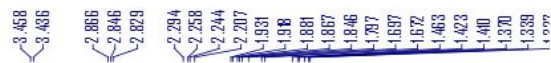

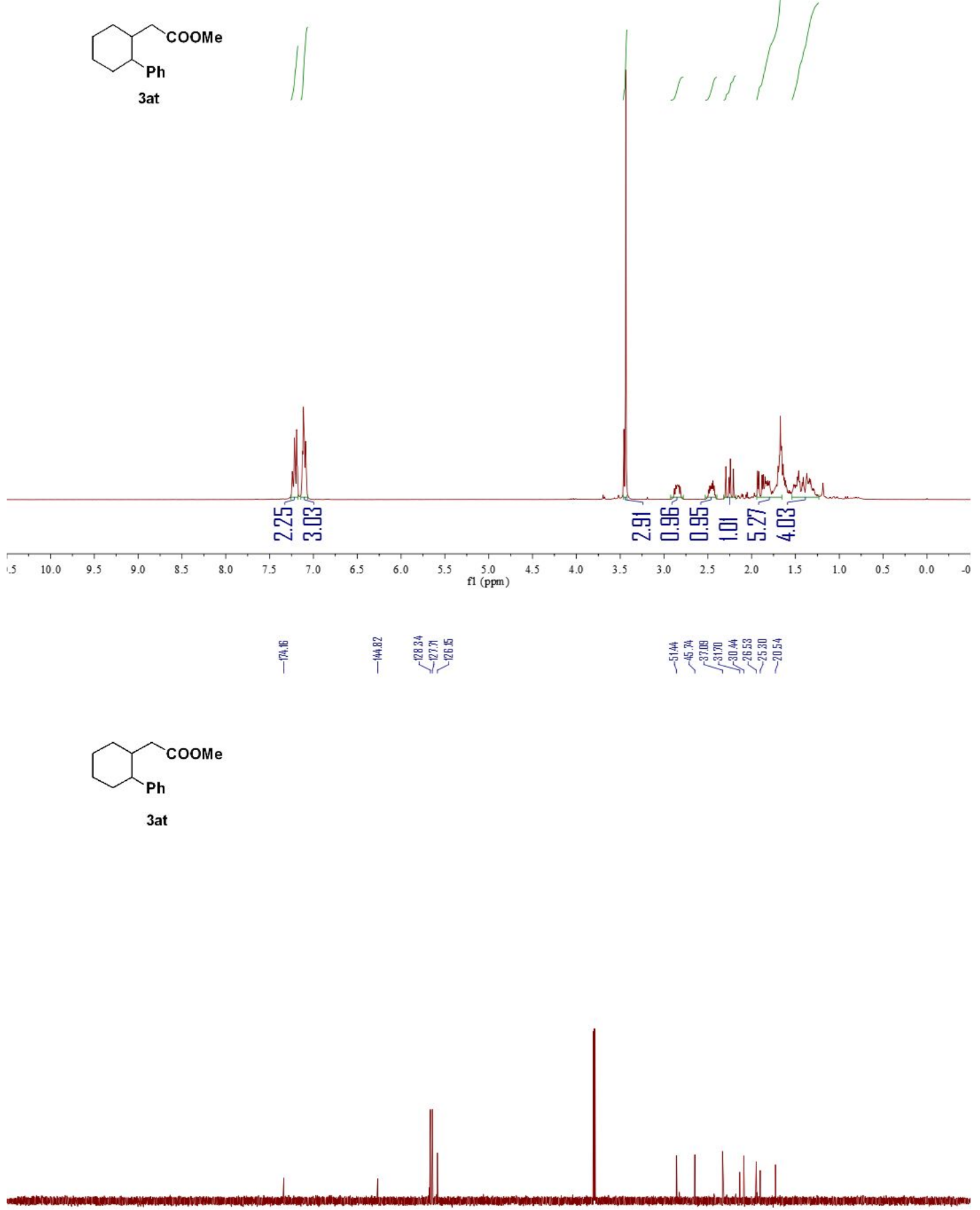

$\begin{array}{lllllllllllllllllllllllllllllllllllllllllllllllllll}60 & 250 & 240 & 230 & 220 & 210 & 200 & 190 & 180 & 170 & 160 & 150 & 140 & 130 & 120 & 110 & 100 & 90 & 80 & 70 & 60 & 50 & 40 & 30 & 20 & 10 & 0 & -10 & -20 & -30 & -4\end{array}$ 


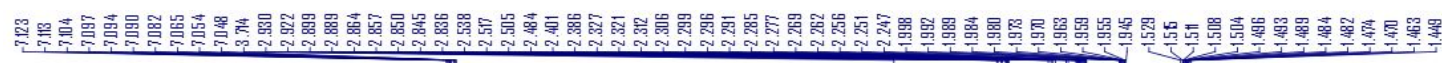
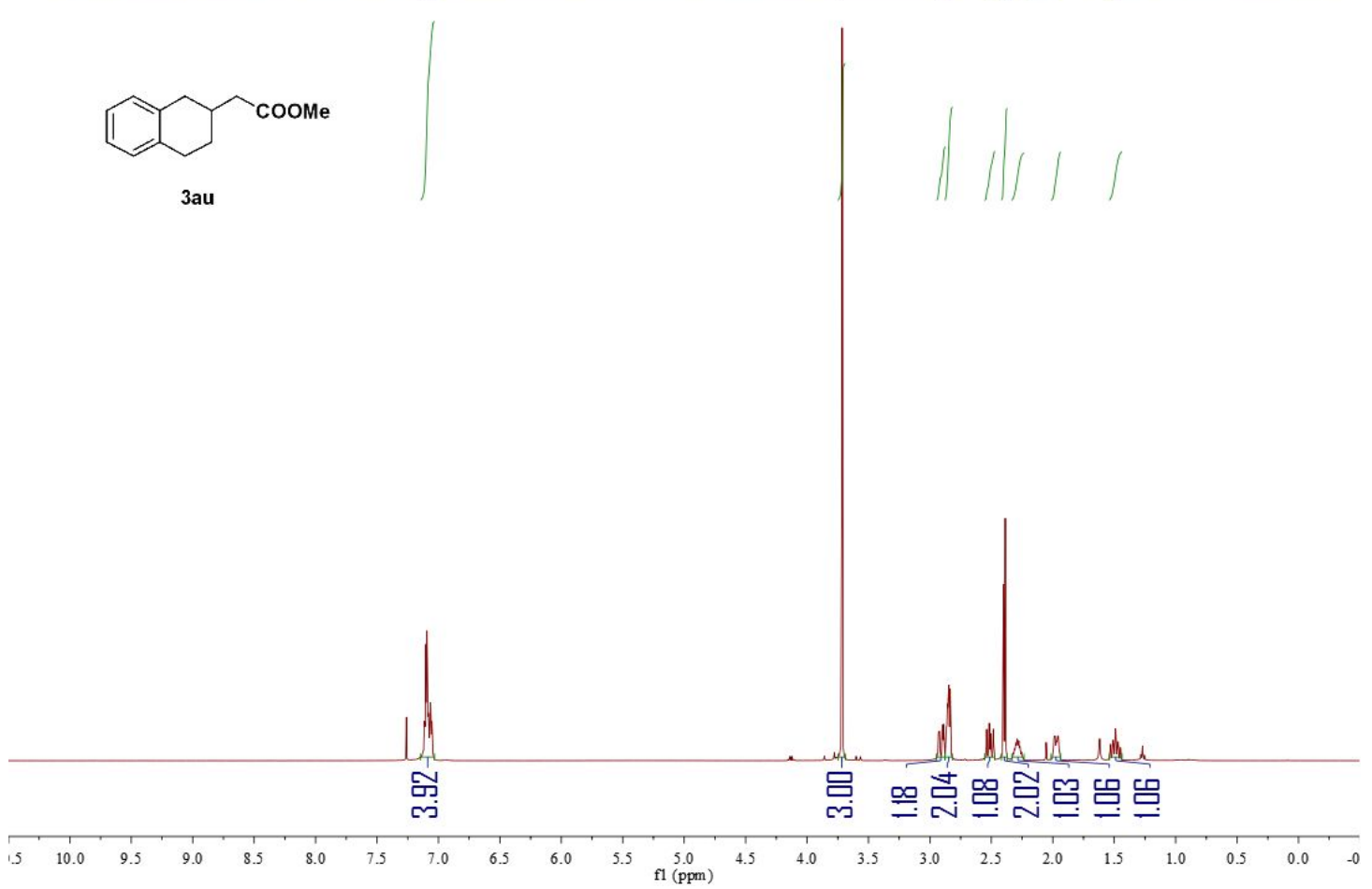

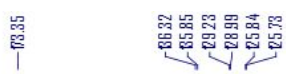

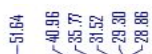

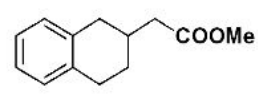

3au

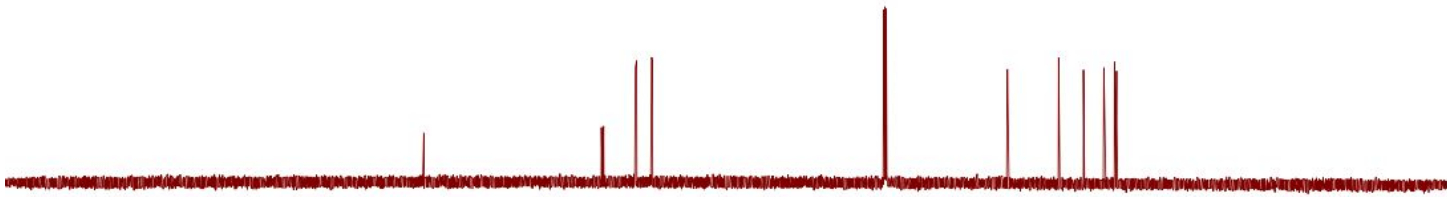

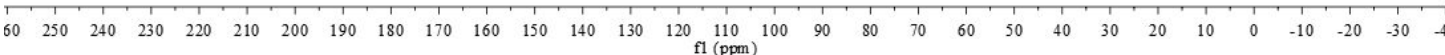



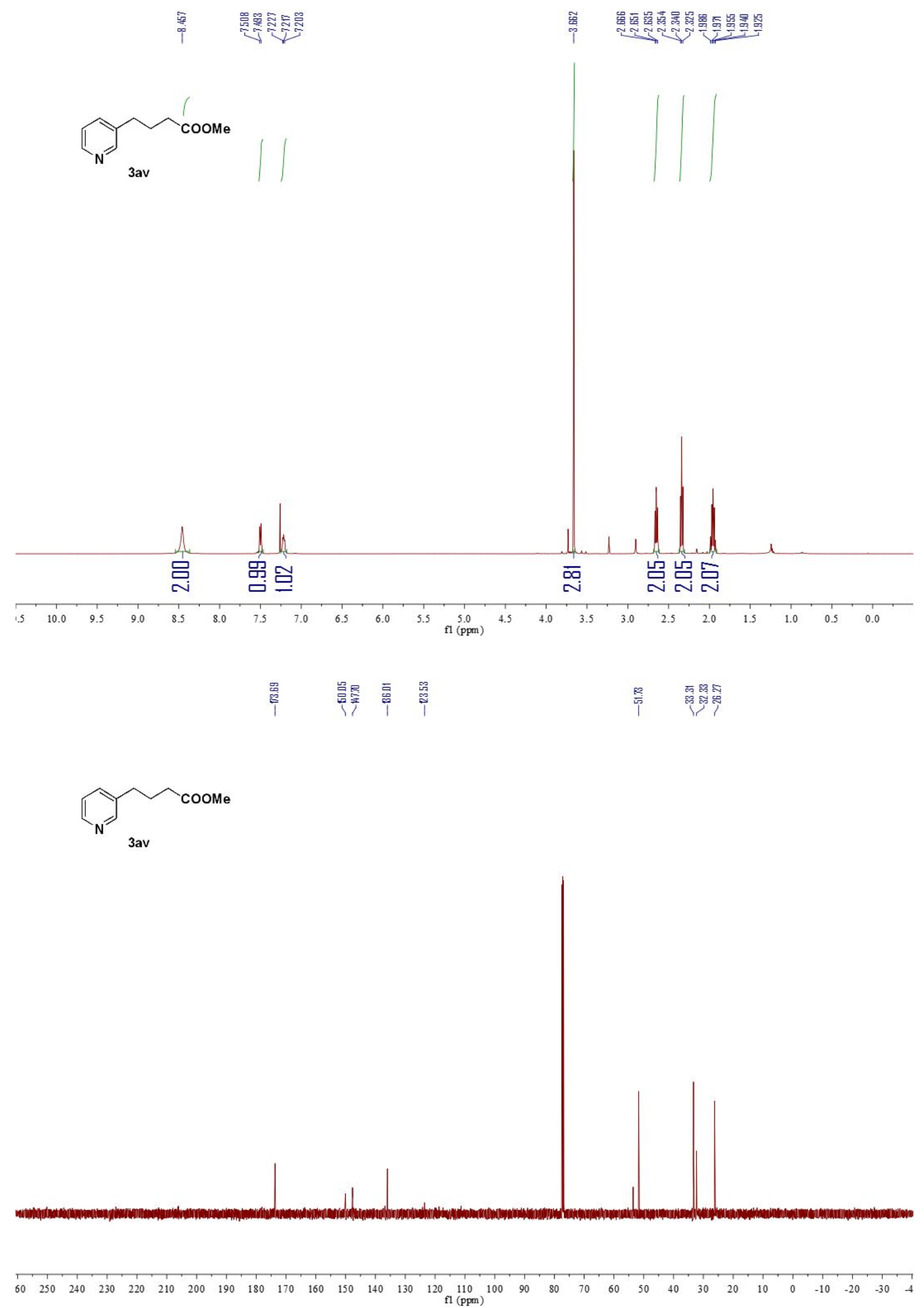


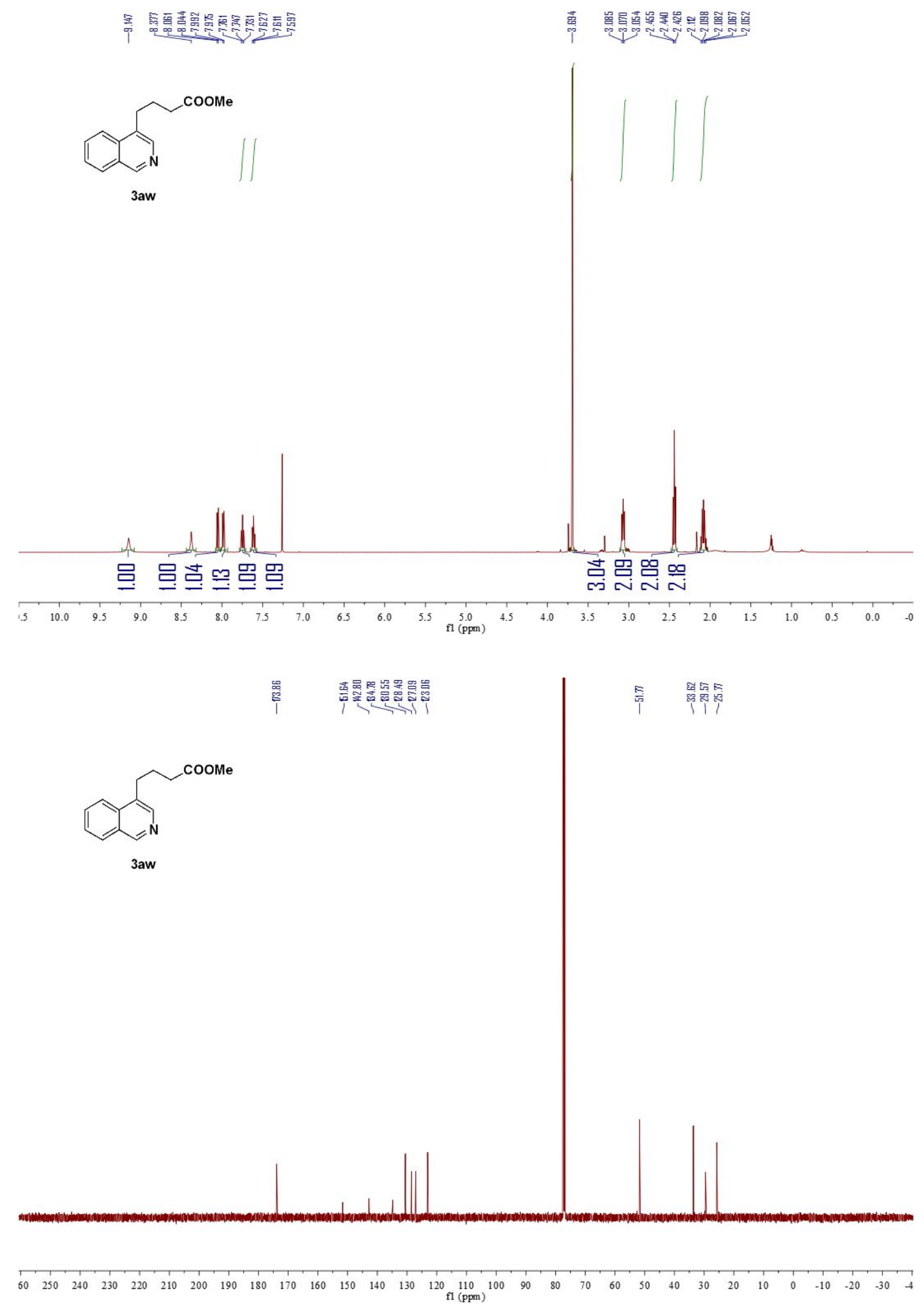




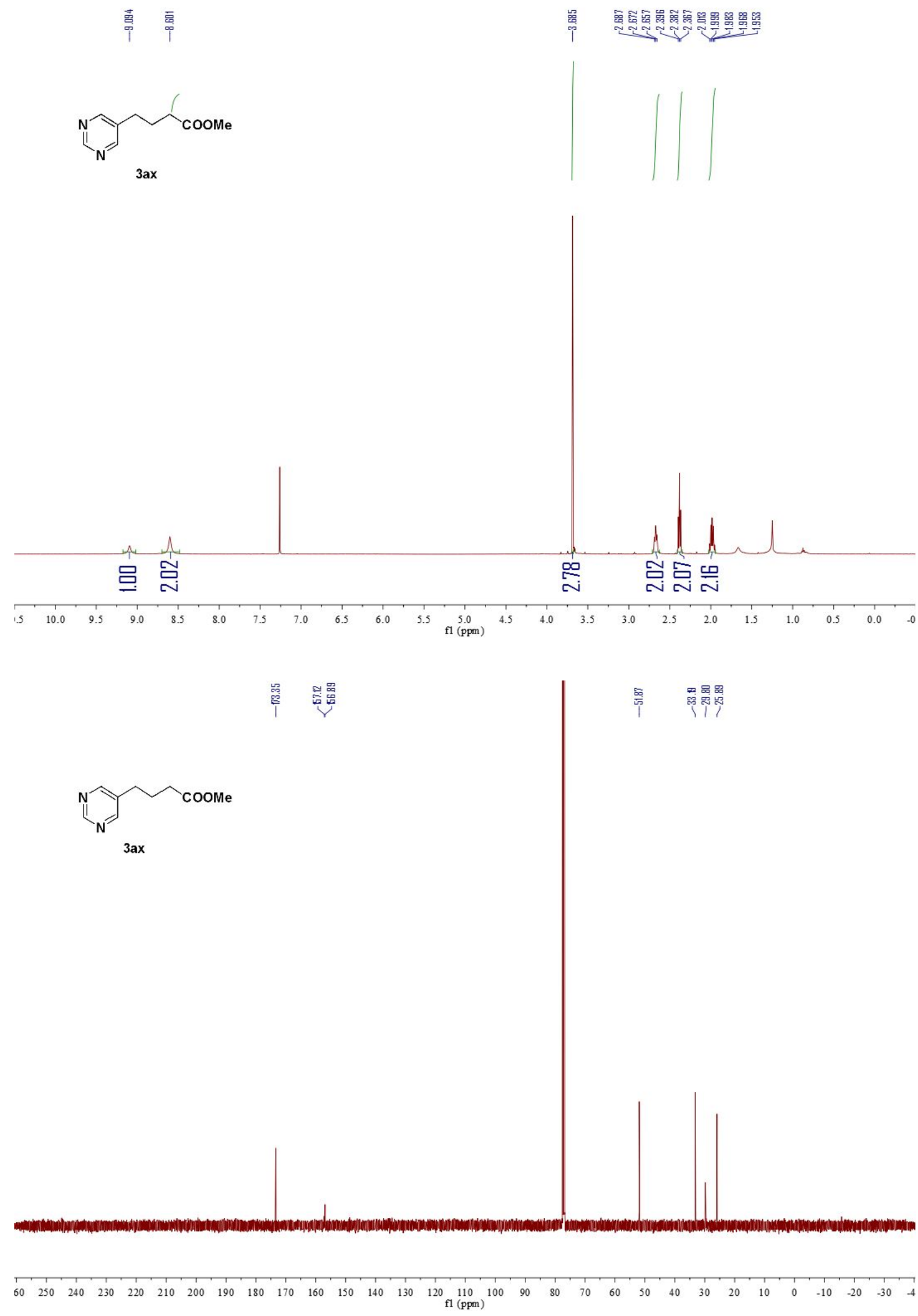




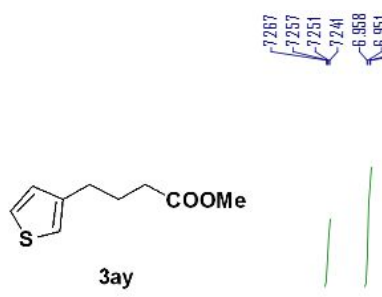

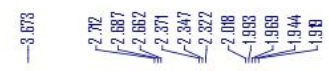
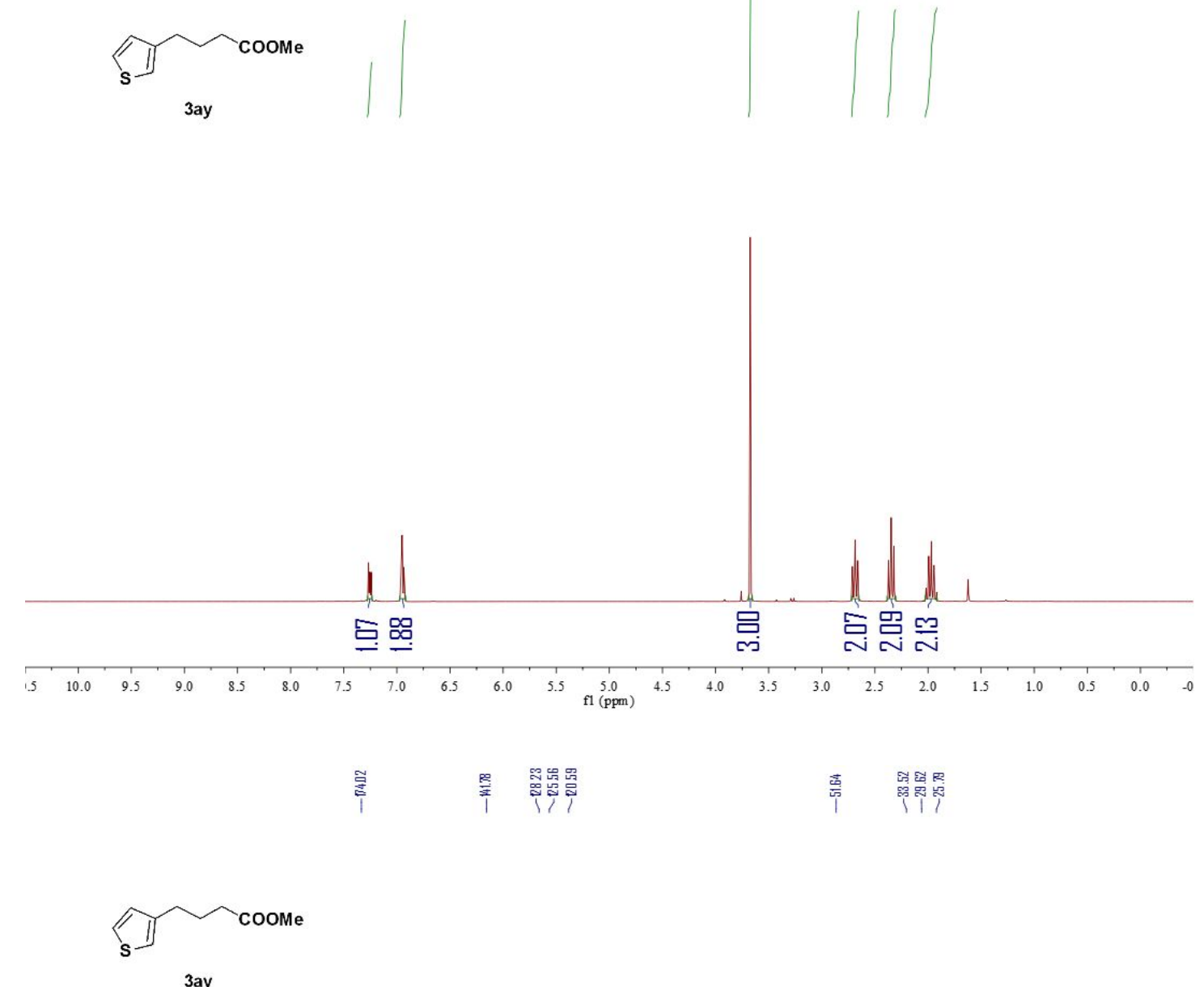

3ay

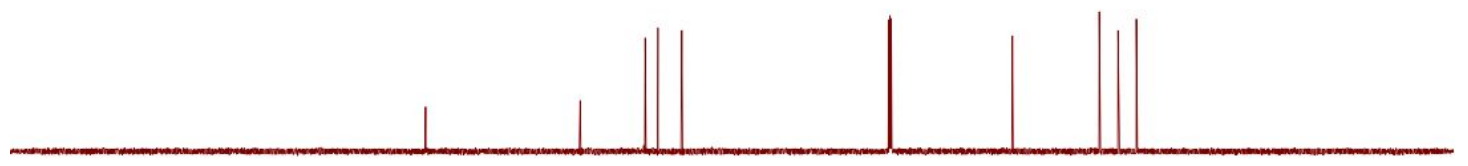

\begin{tabular}{llllllllllllllllllllllllllllllllllllllllllllllllllll}
\hline 60 & 250 & 240 & 230 & 220 & 210 & 200 & 190 & 180 & 170 & 160 & 150 & 140 & 130 & 120 & 110 & 100 & 90 & 80 & 70 & 60 & 50 & 40 & 30 & 20 & 10 & 0 & -10 & -20 & -30 & -4
\end{tabular} 


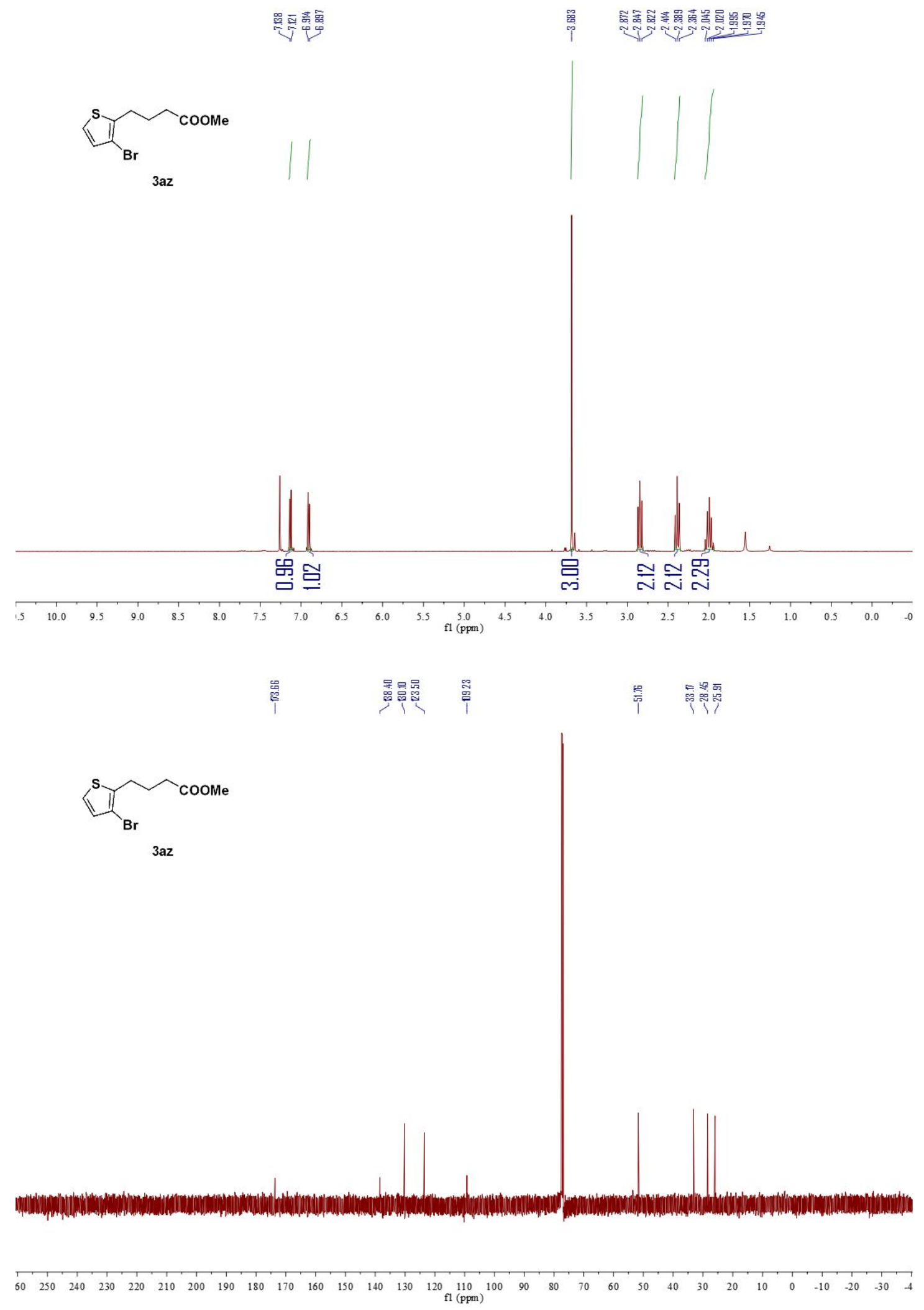




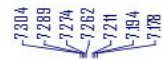

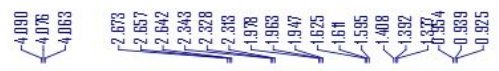
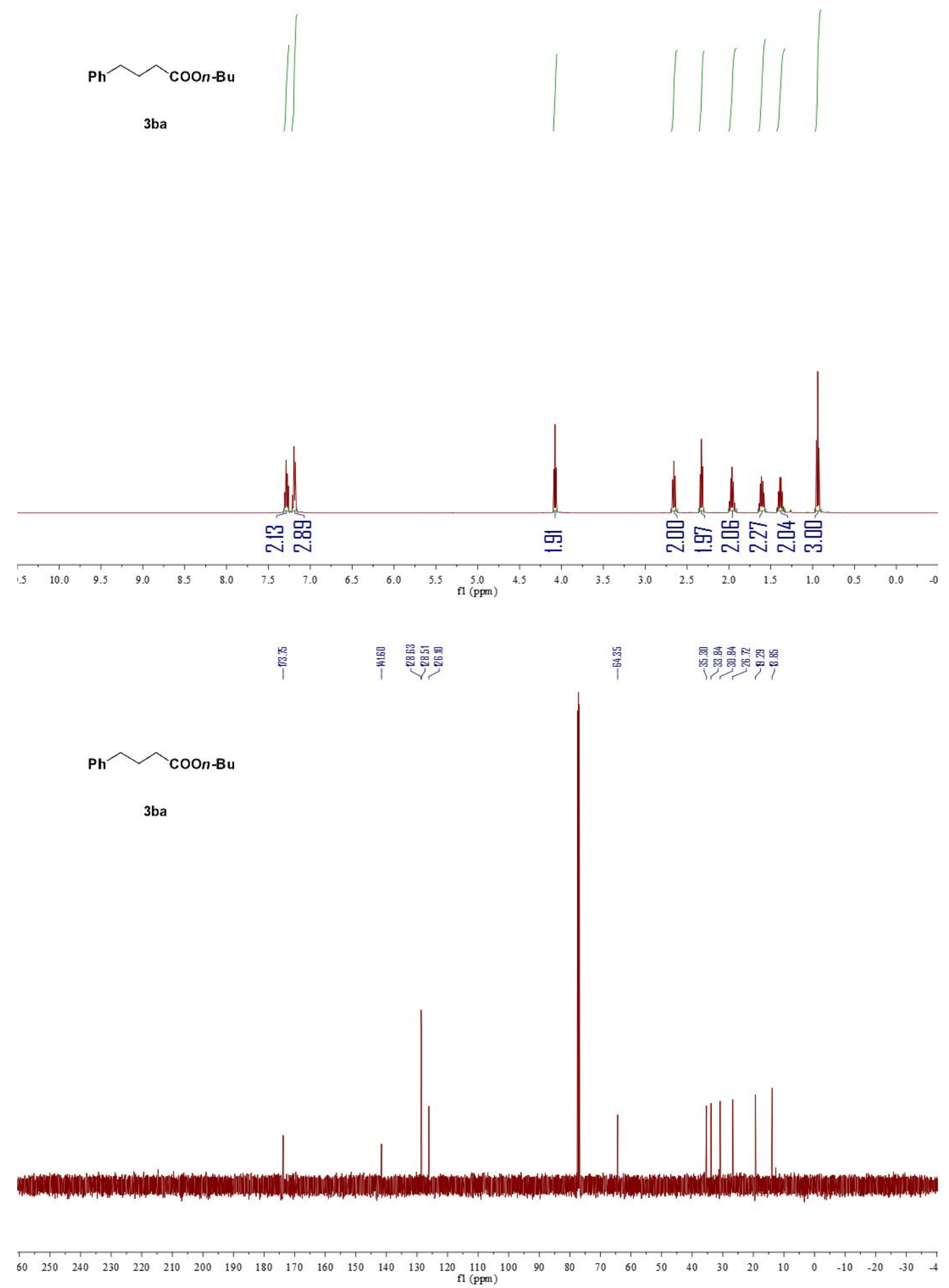


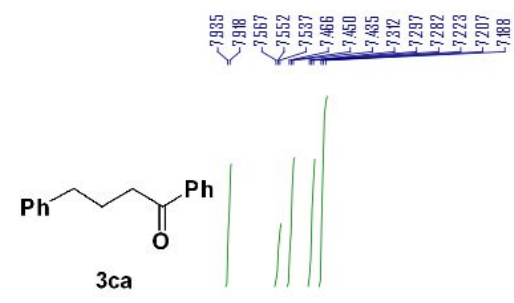

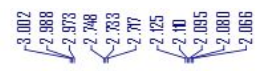
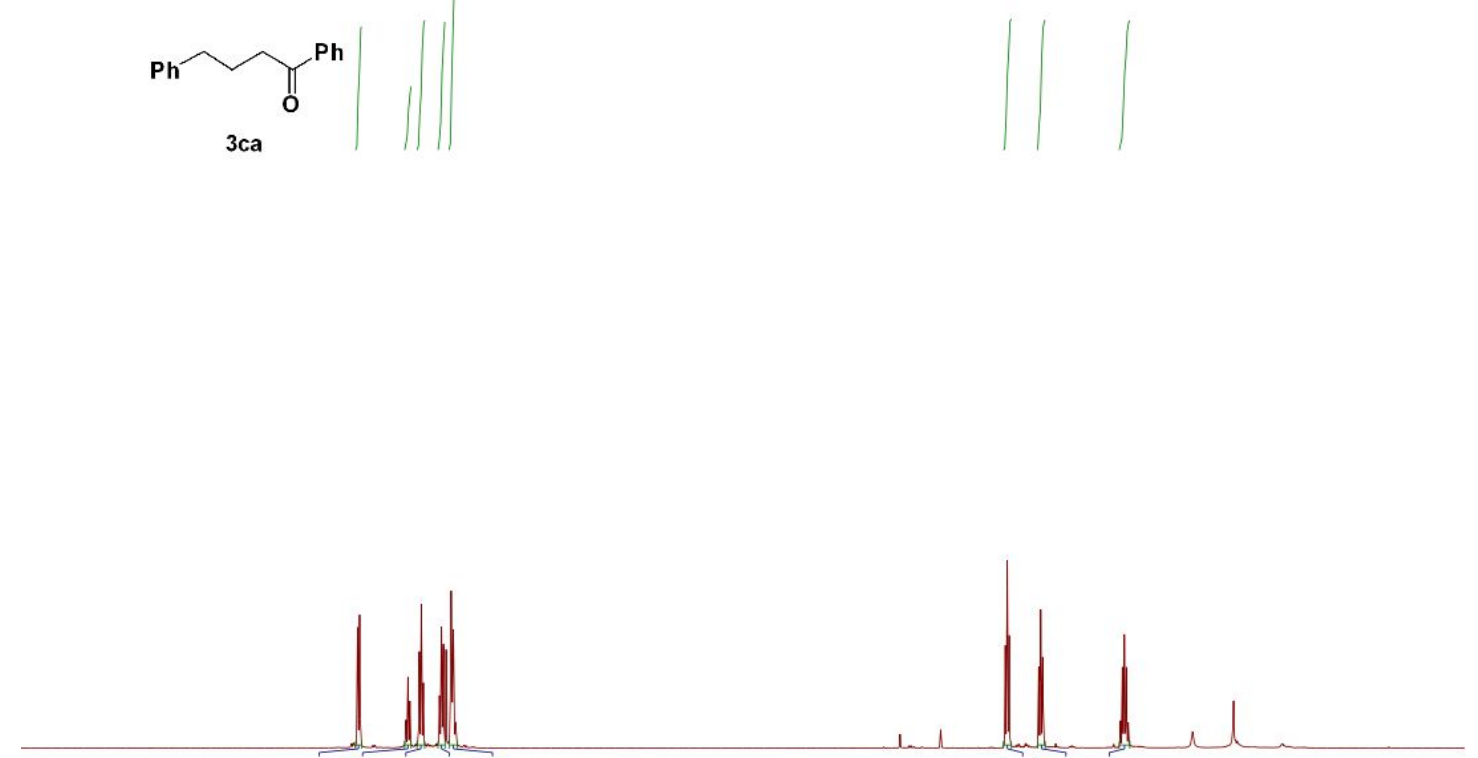

器骂哭点虽

怘器器

\begin{tabular}{llllllllllllllllllllllllll}
\hline .5 & 10.0 & 9.5 & 9.0 & 8.5 & 8.0 & 7.5 & 7.0 & 6.5 & 6.0 & 5.5 & $\begin{array}{c}1 \\
\mathrm{f}\end{array} .0$ & 4.5 & 4.0 & 3.5 & 3.0 & 2.5 & 2.0 & 1.5 & 1.0 & 0.5 & 0.0 & -0
\end{tabular}

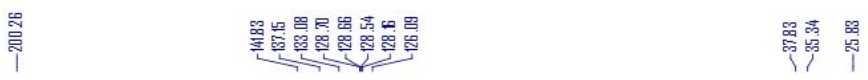

$\prod_{3 \mathrm{Ph}}^{\mathrm{Ph}}$

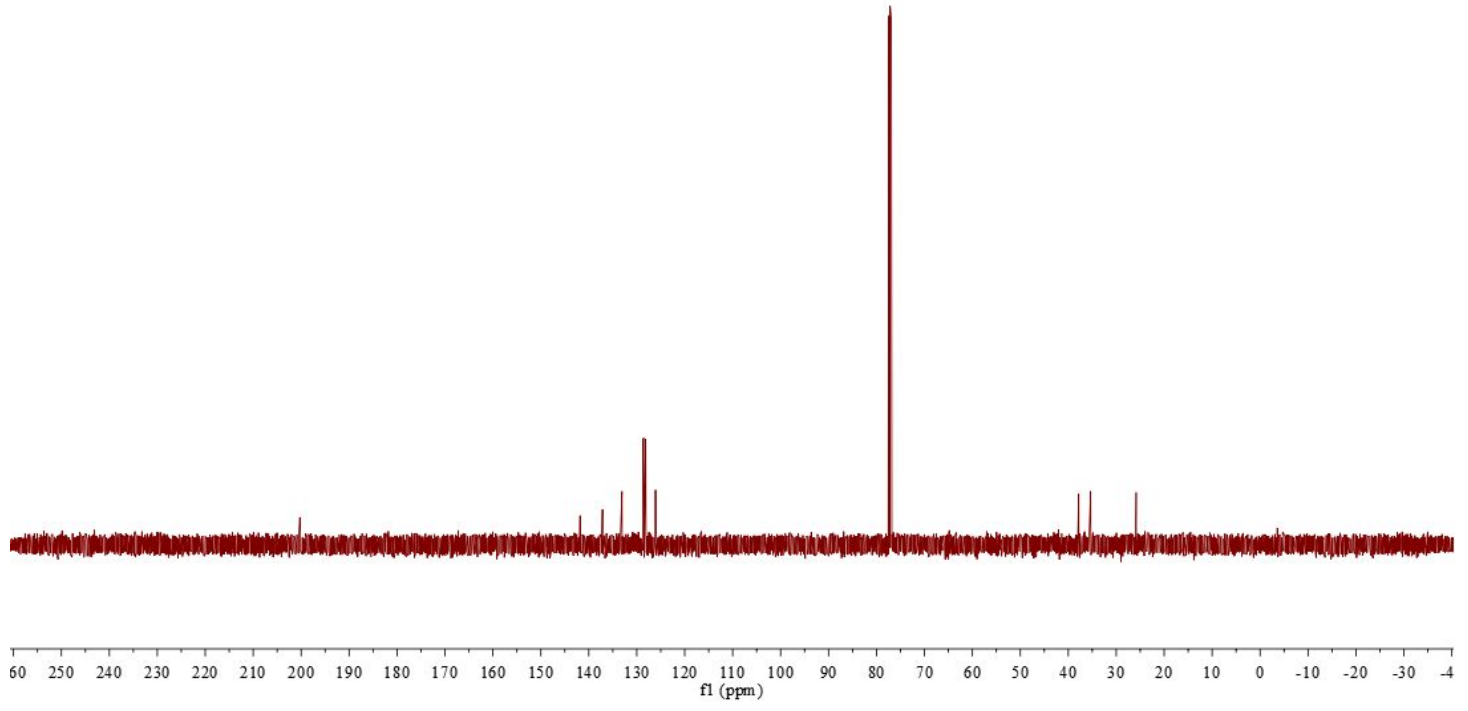




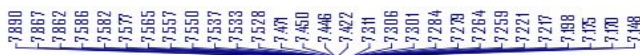

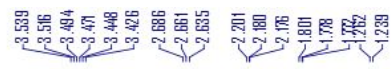
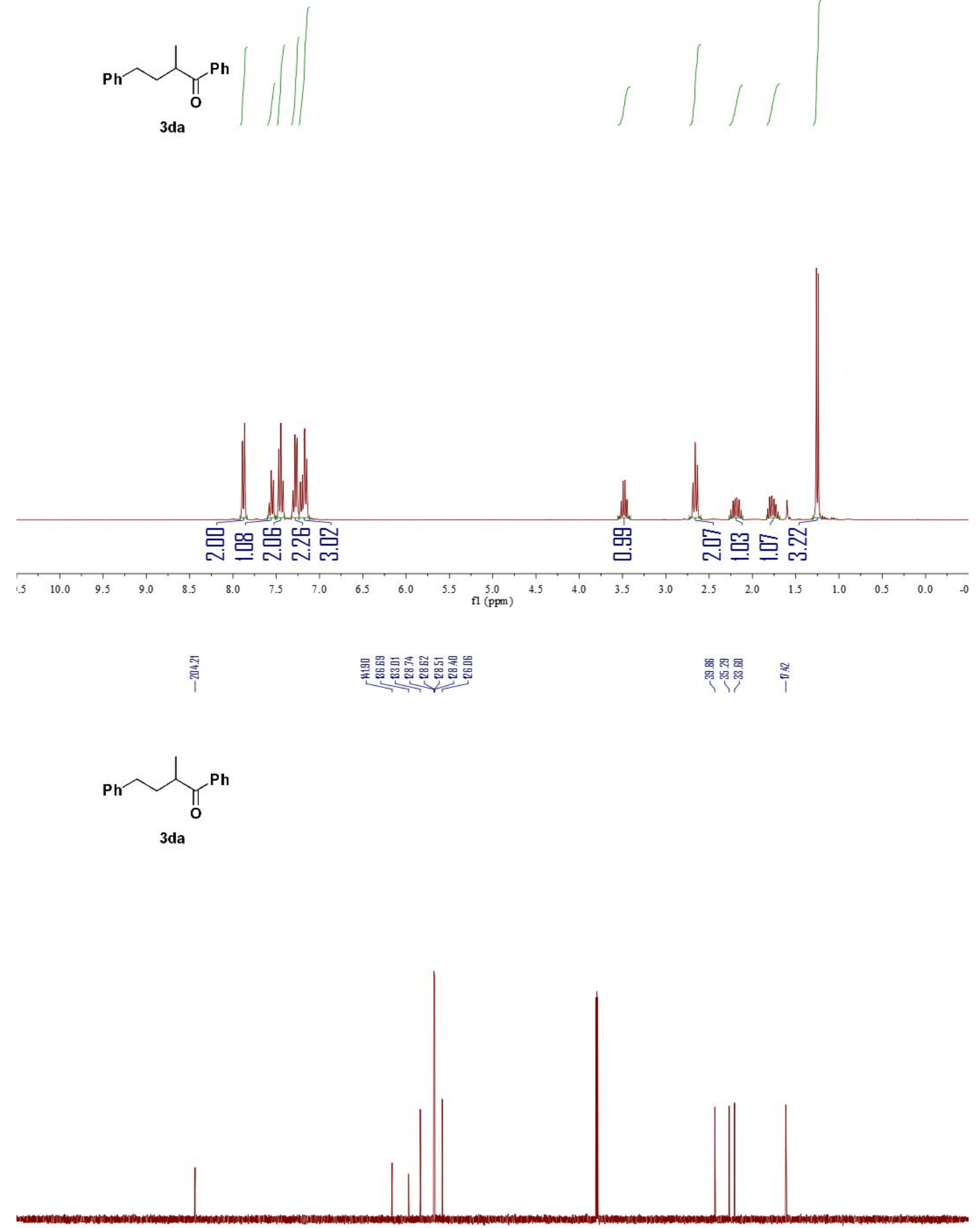

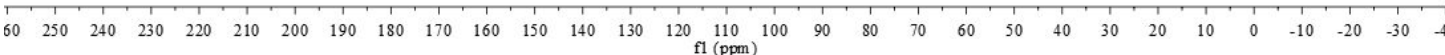




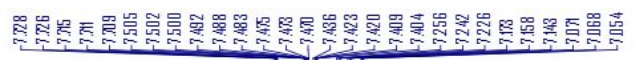

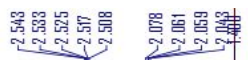

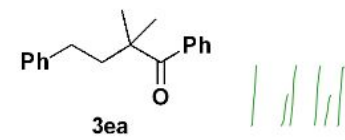
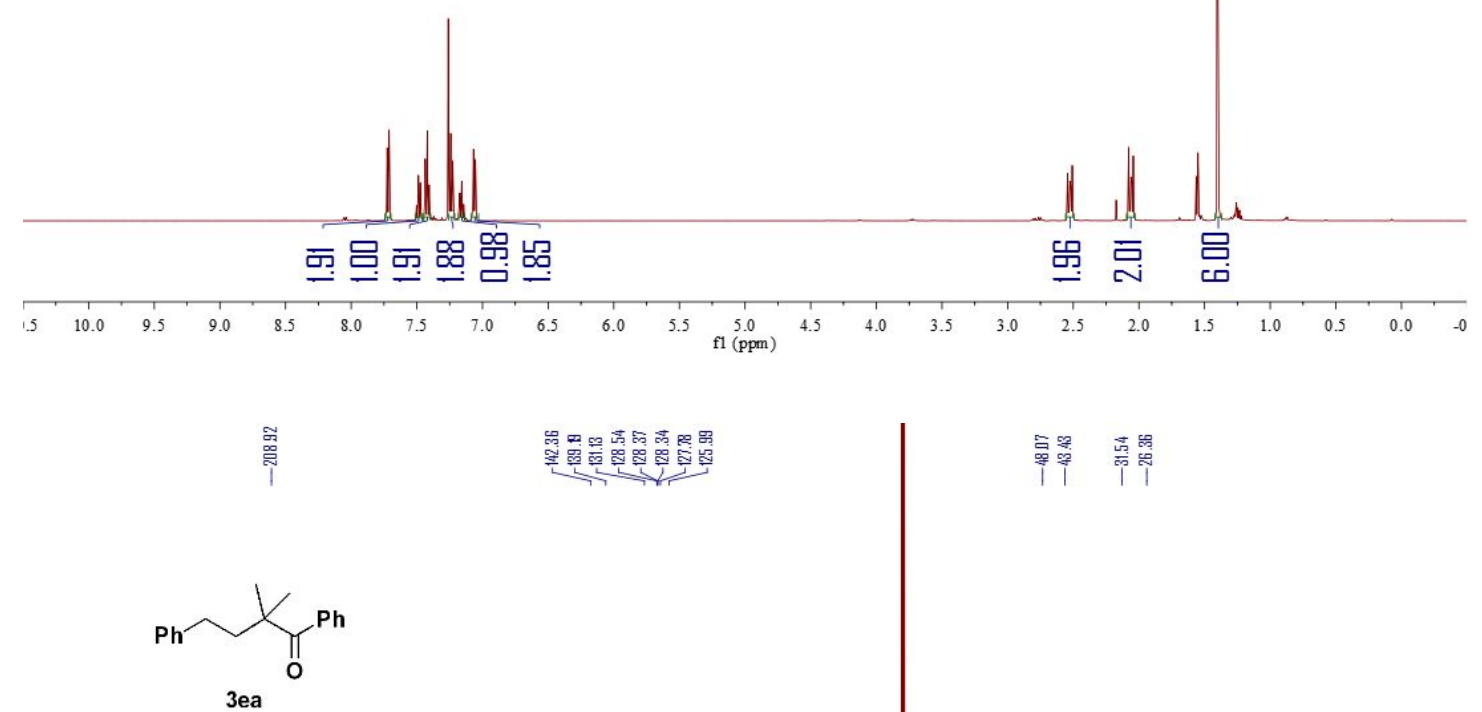

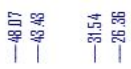

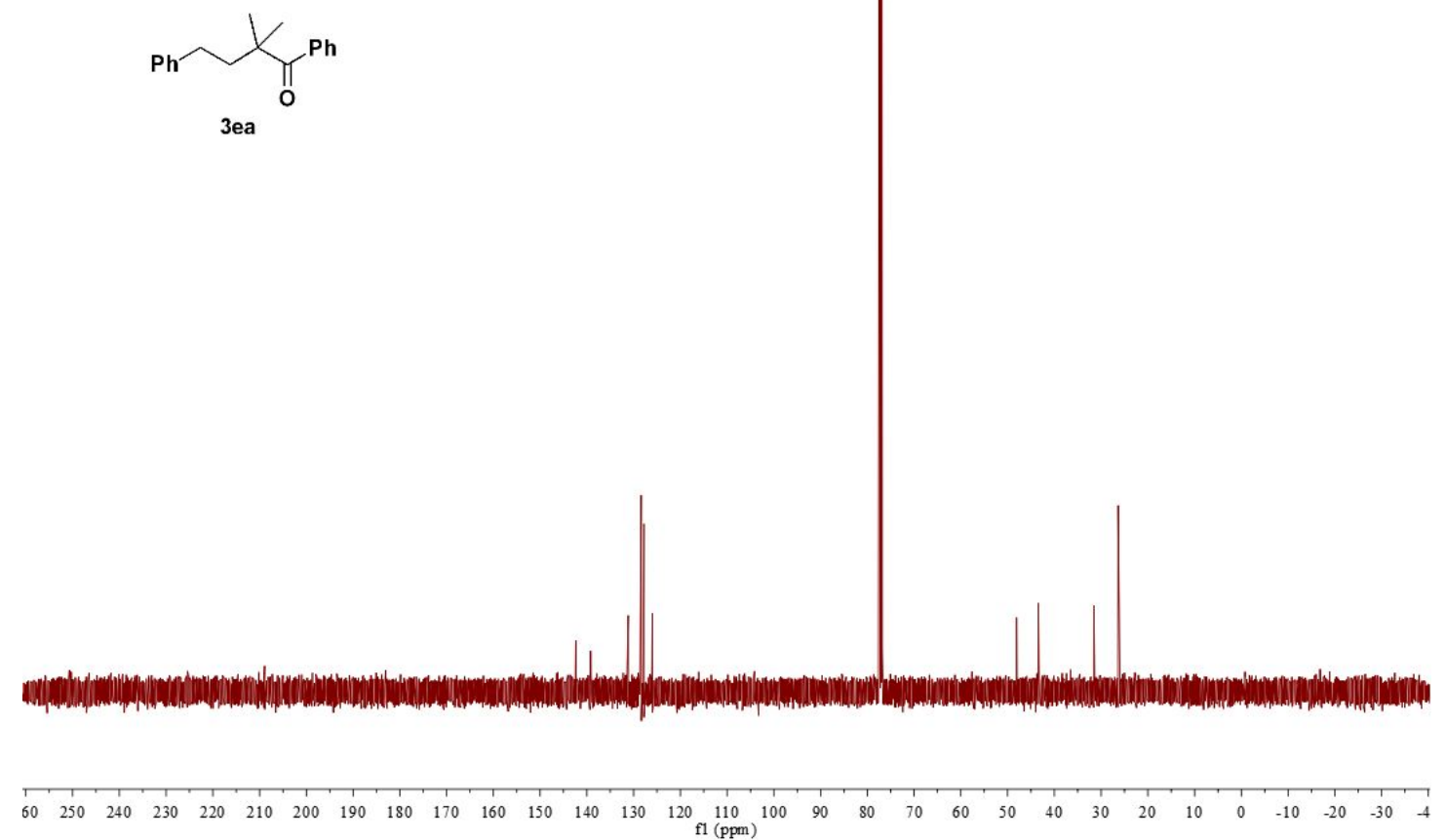




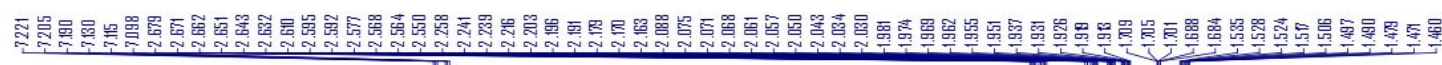<smiles>O=C1CCCC1CCc1ccccc1</smiles>

$3 f a$
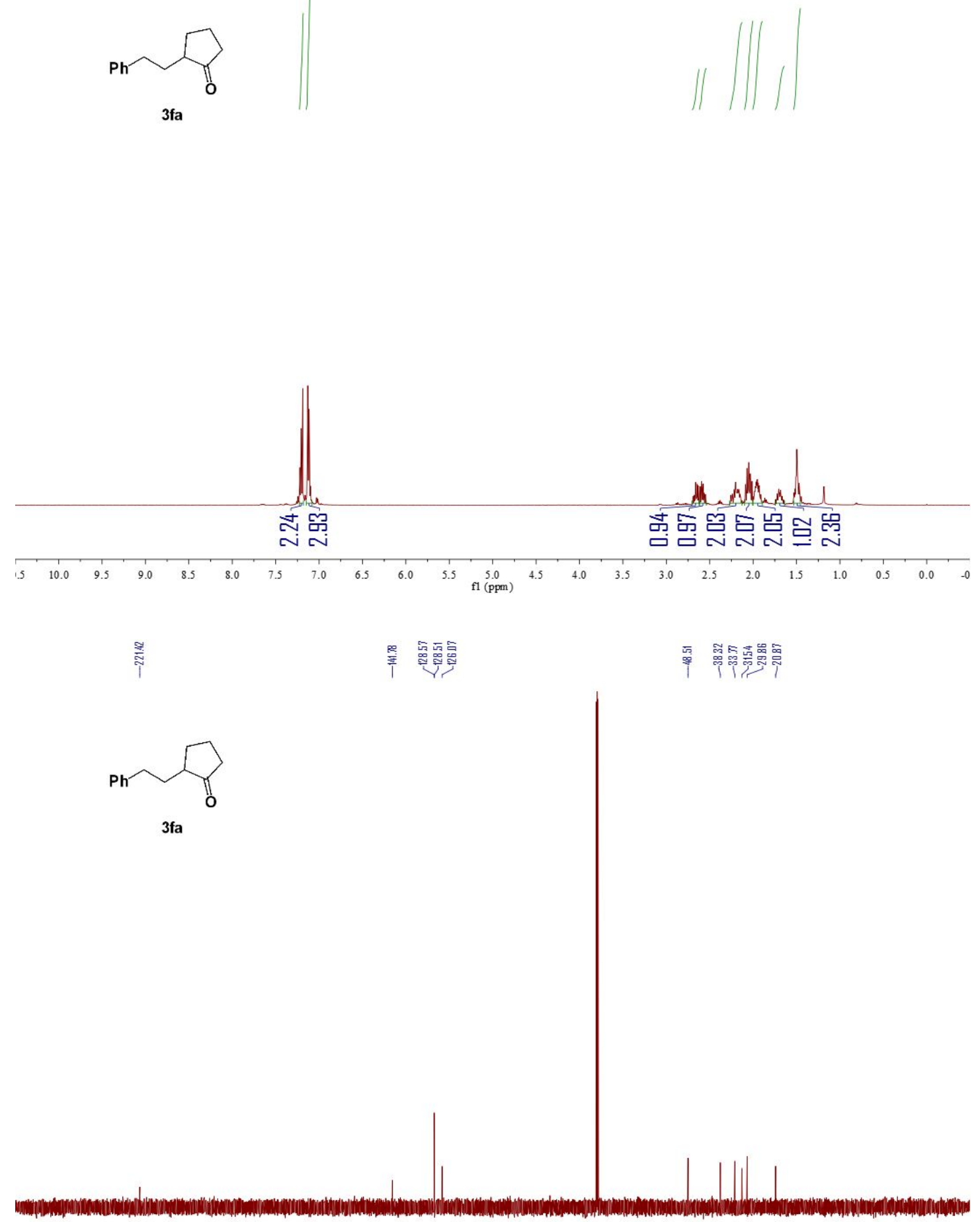

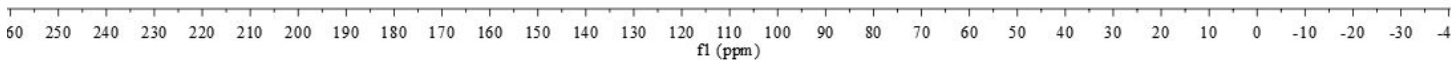




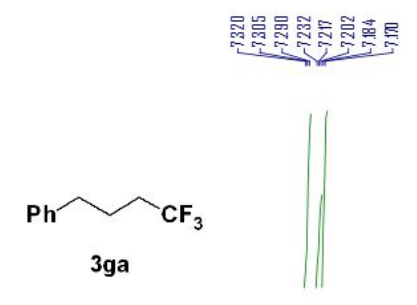

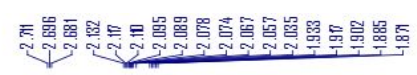
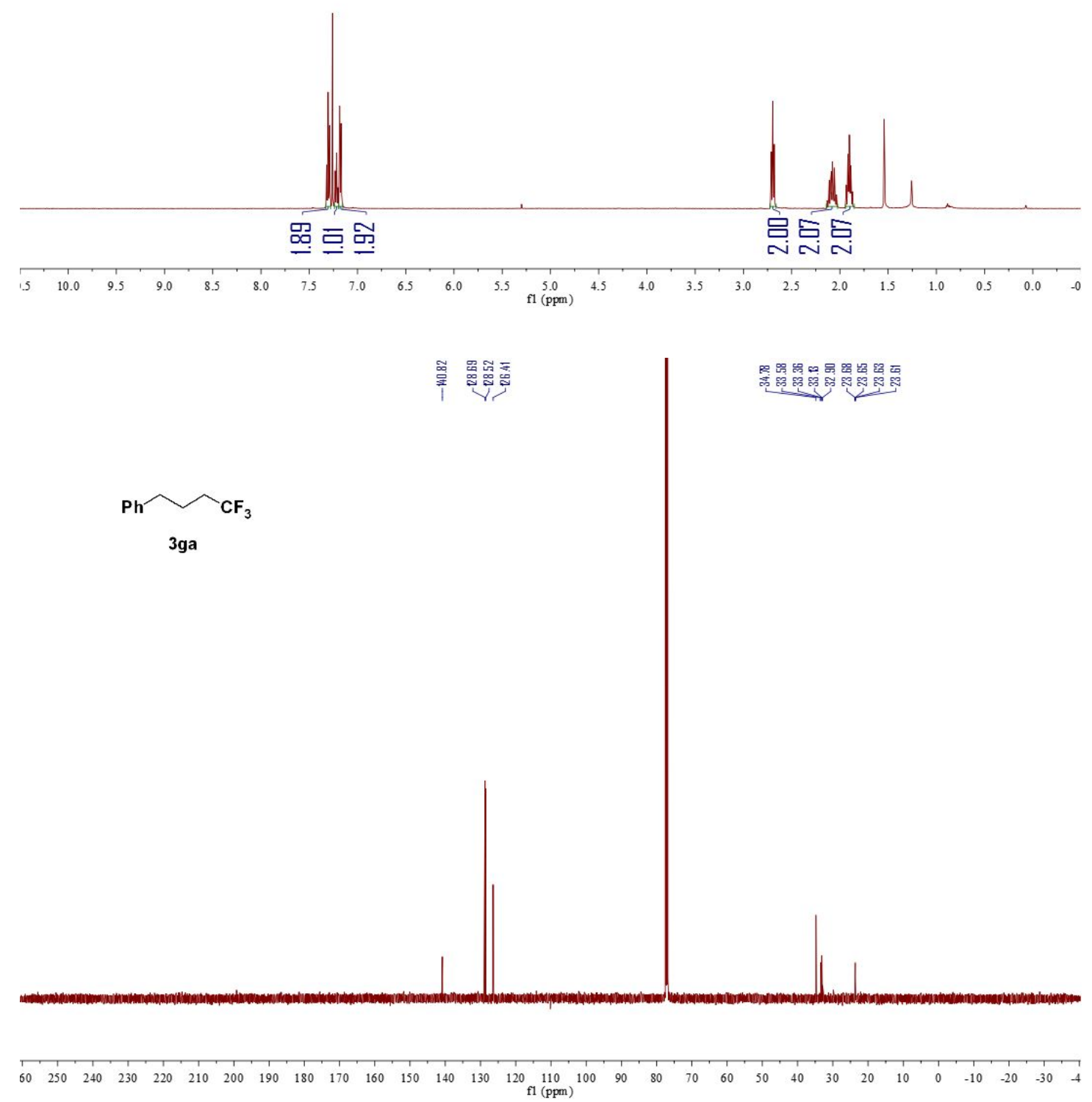
$\mathrm{Ph} \frown \mathrm{CF}_{3}$

3ga

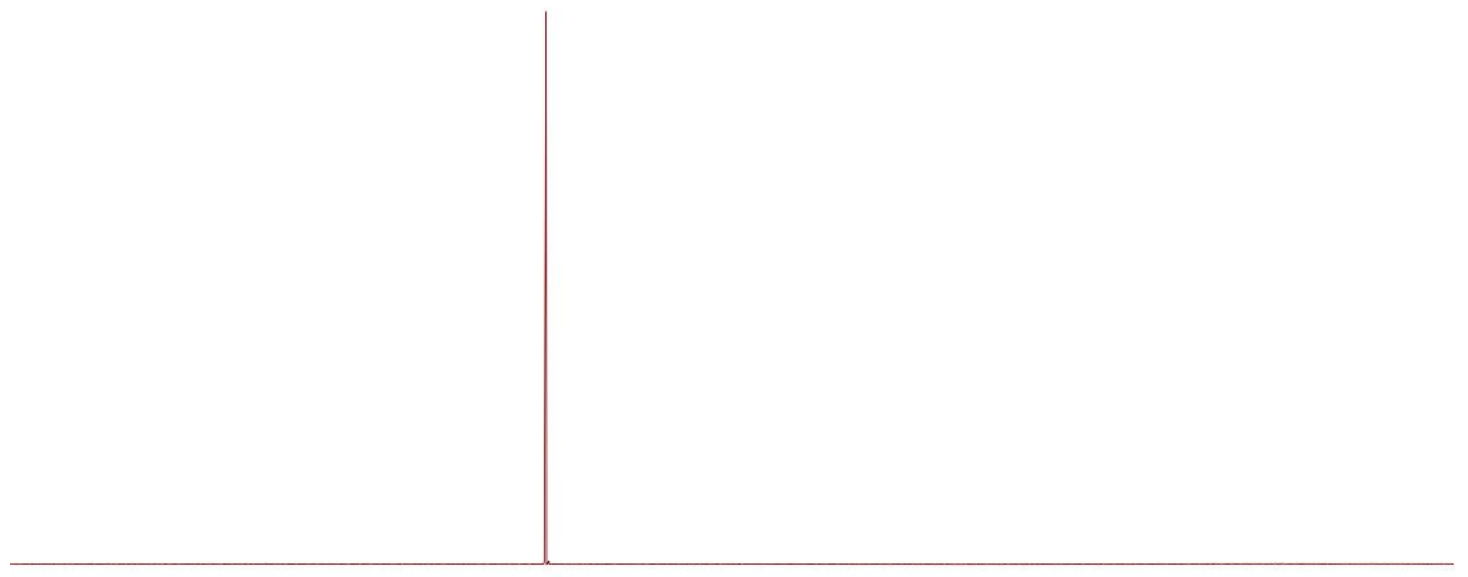

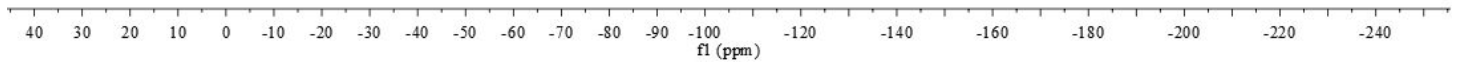

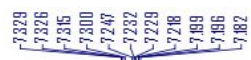

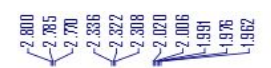
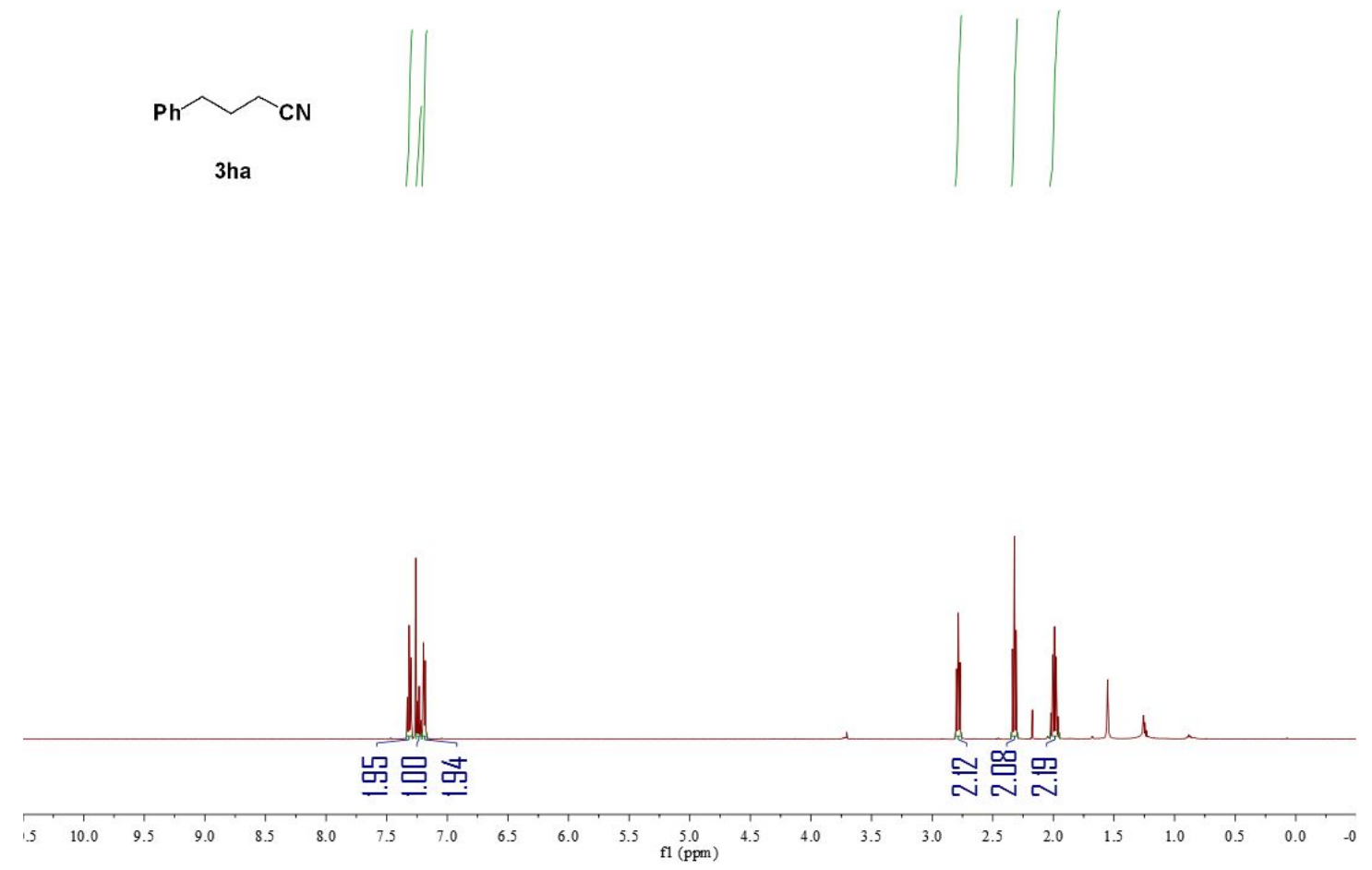


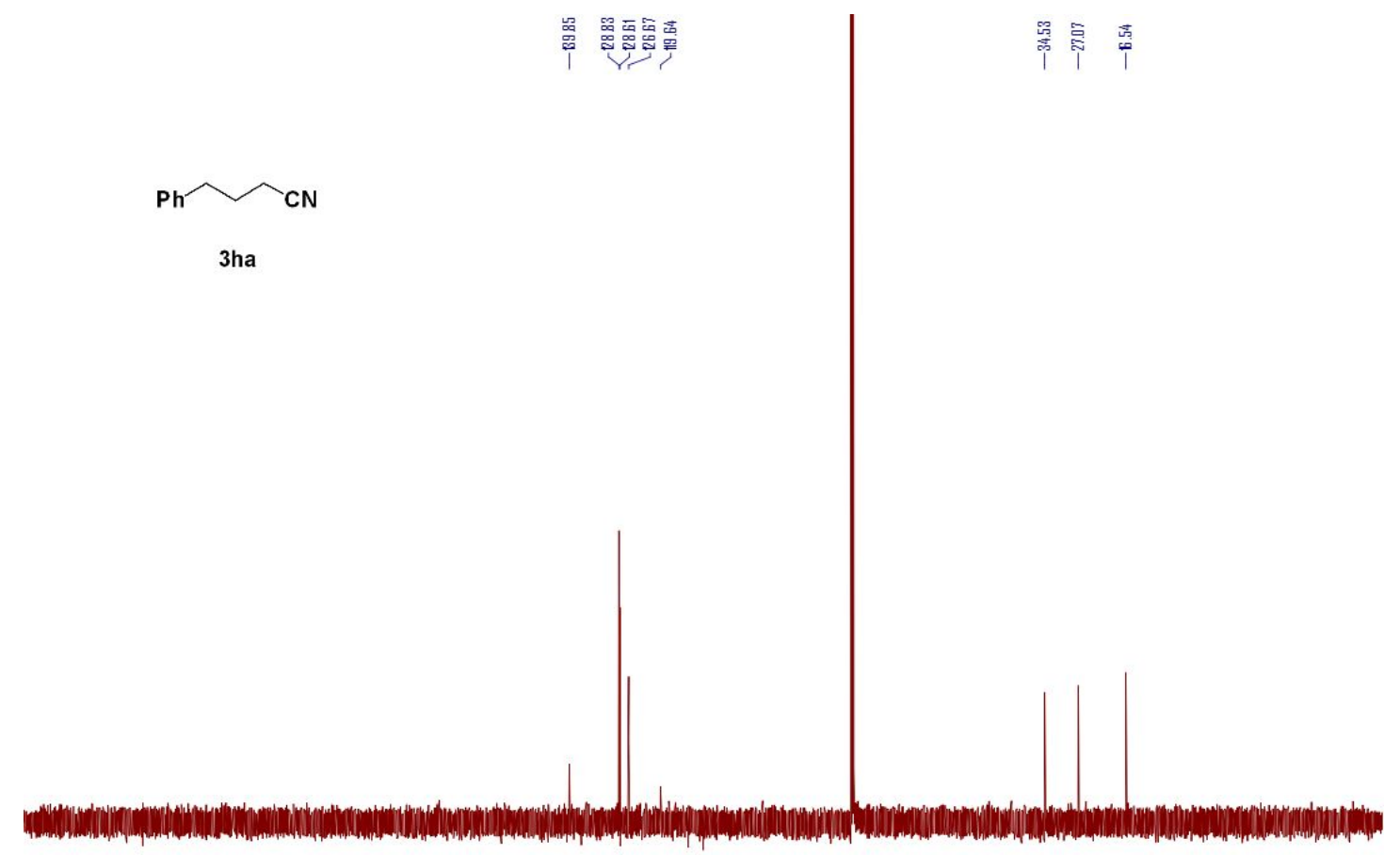

\begin{tabular}{llllllllllllllllllllllllllllllllllllll}
\hline 60 & 250 & 240 & 230 & 220 & 210 & 200 & 190 & 180 & 170 & 160 & 150 & 140 & 130 & 120 & 110 & 100 & 90 & 80 & 70 & 60 & 50 & 40 & 30 & 20 & 10 & 0 & -10 & -20 & -30 & -4
\end{tabular}
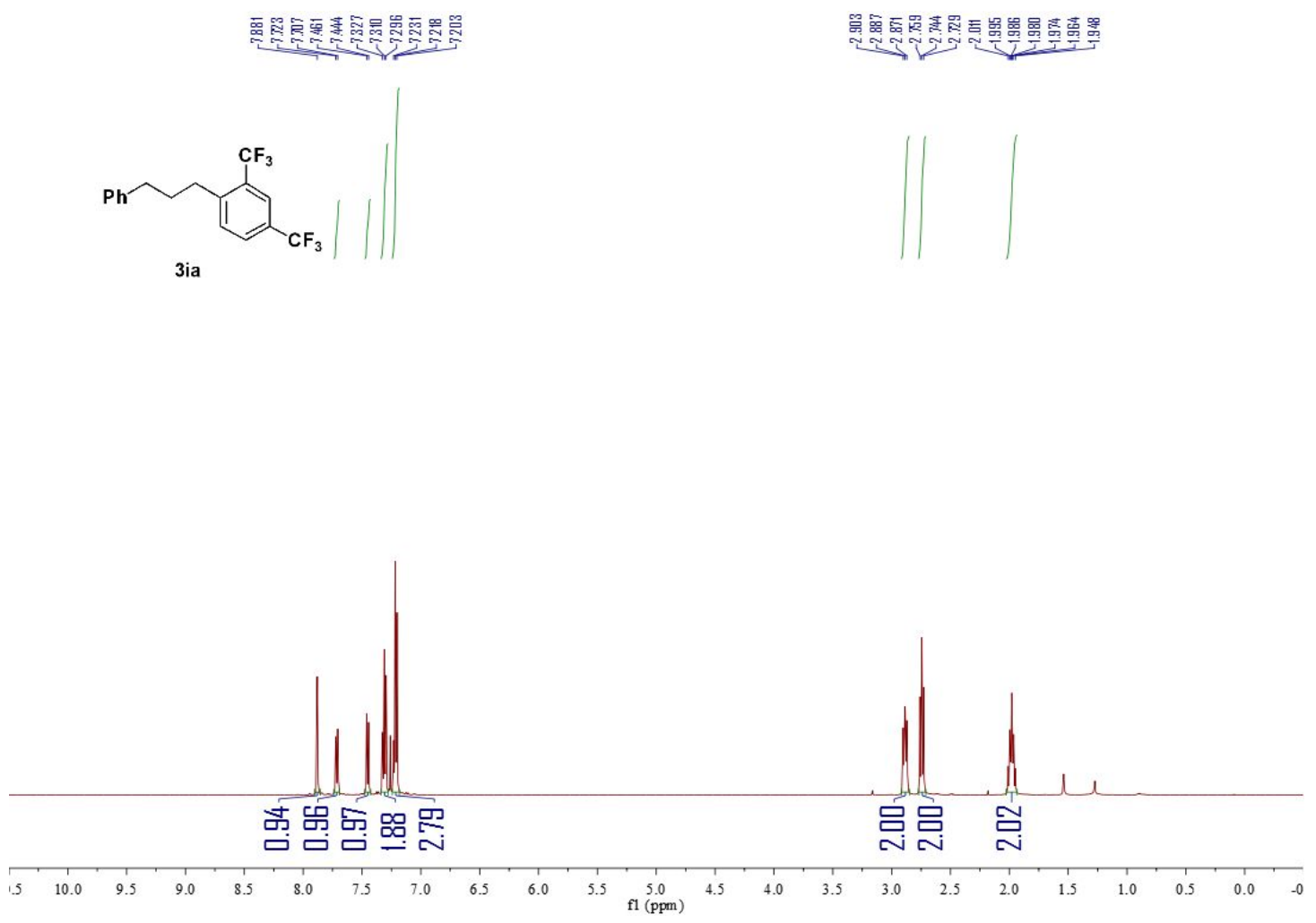


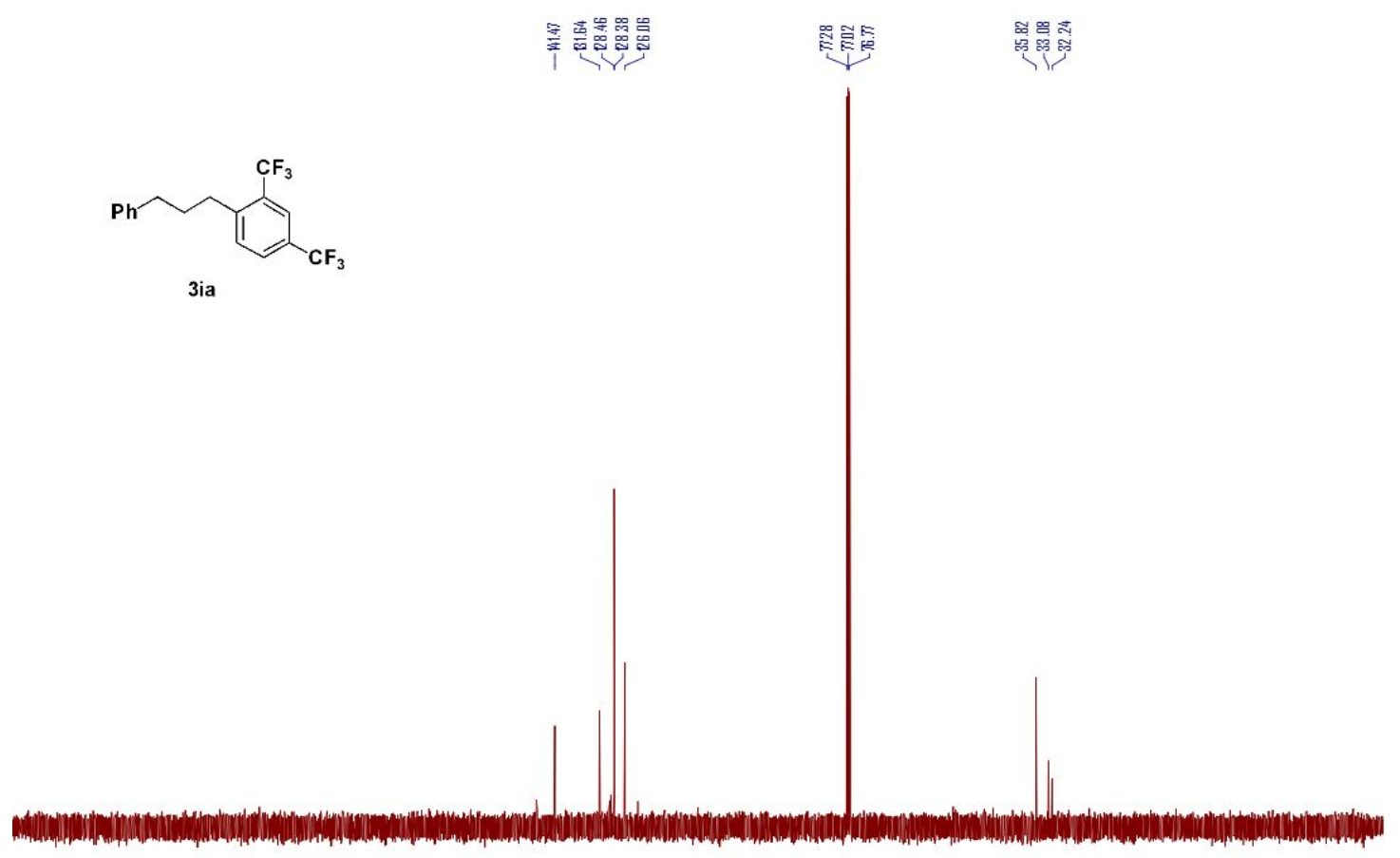

$\begin{array}{lllllllllllllllllllllllllllllllllllllll}6 & 250 & 240 & 230 & 220 & 210 & 200 & 190 & 180 & 170 & 160 & 150 & 140 & 130 & 120 & 110 & 100 & 90 & 80 & 70 & 60 & 50 & 40 & 30 & 20 & 10 & 0 & -10 & -20 & -30 & -4\end{array}$

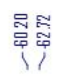
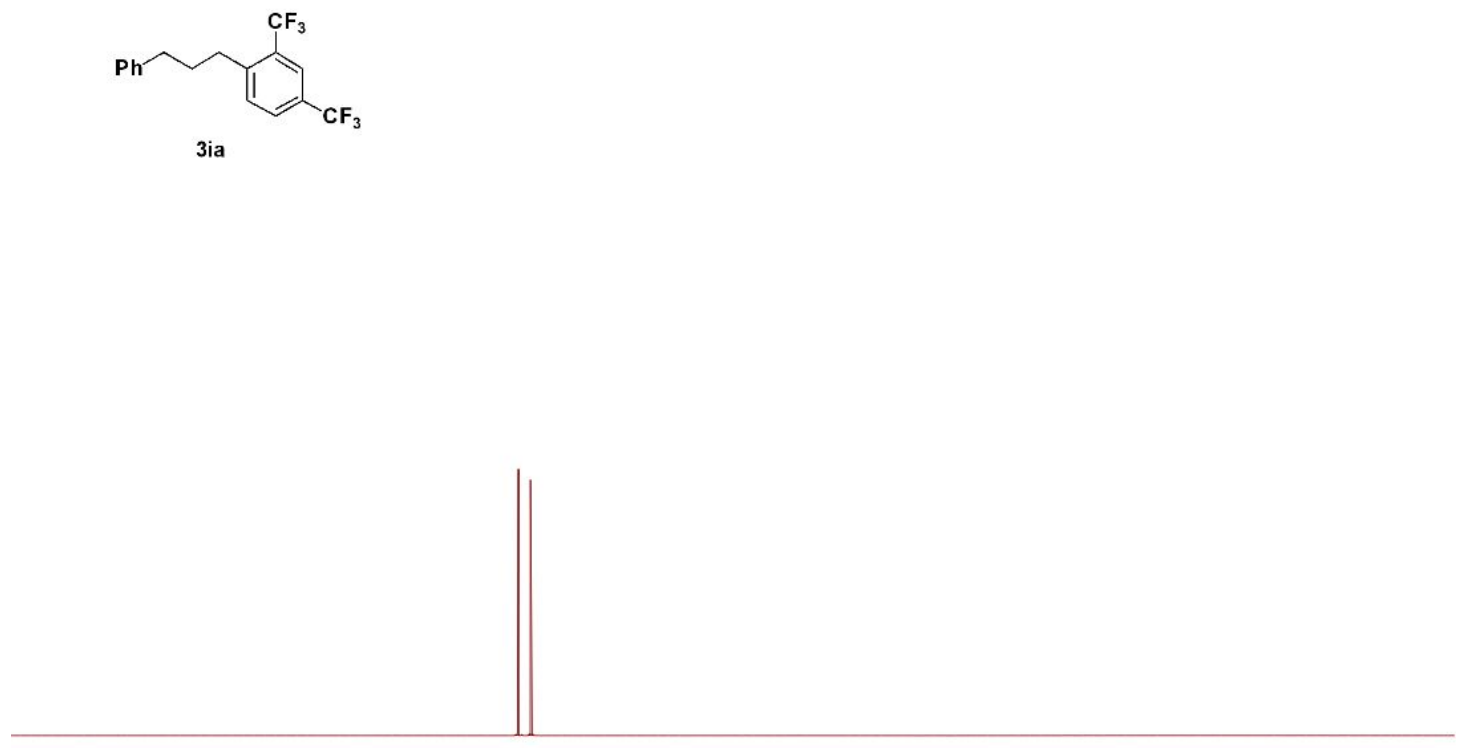

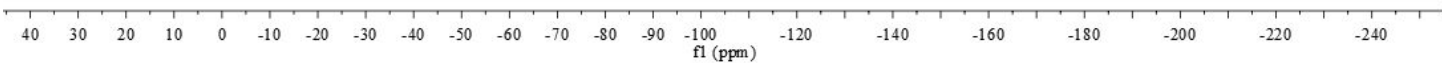



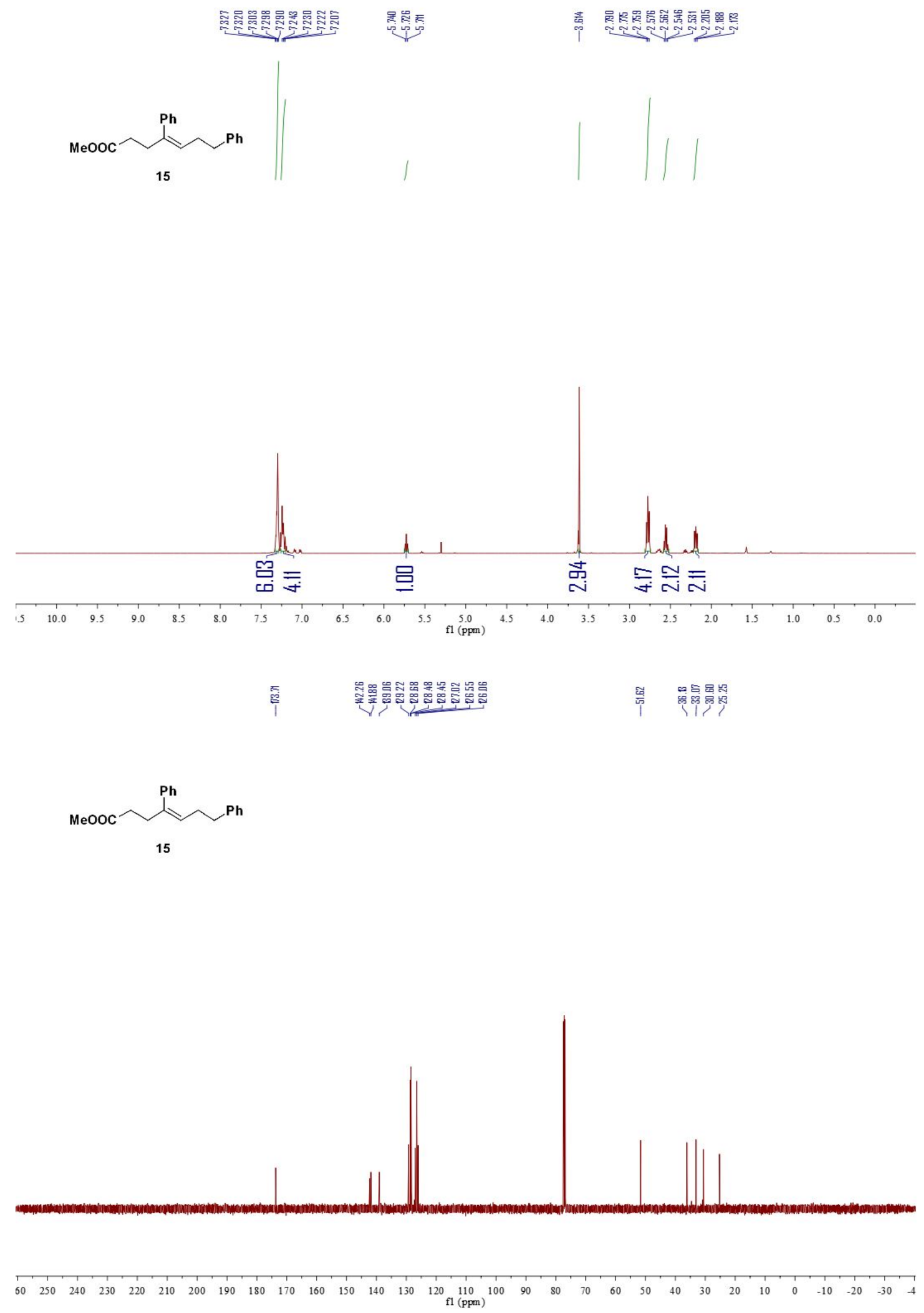


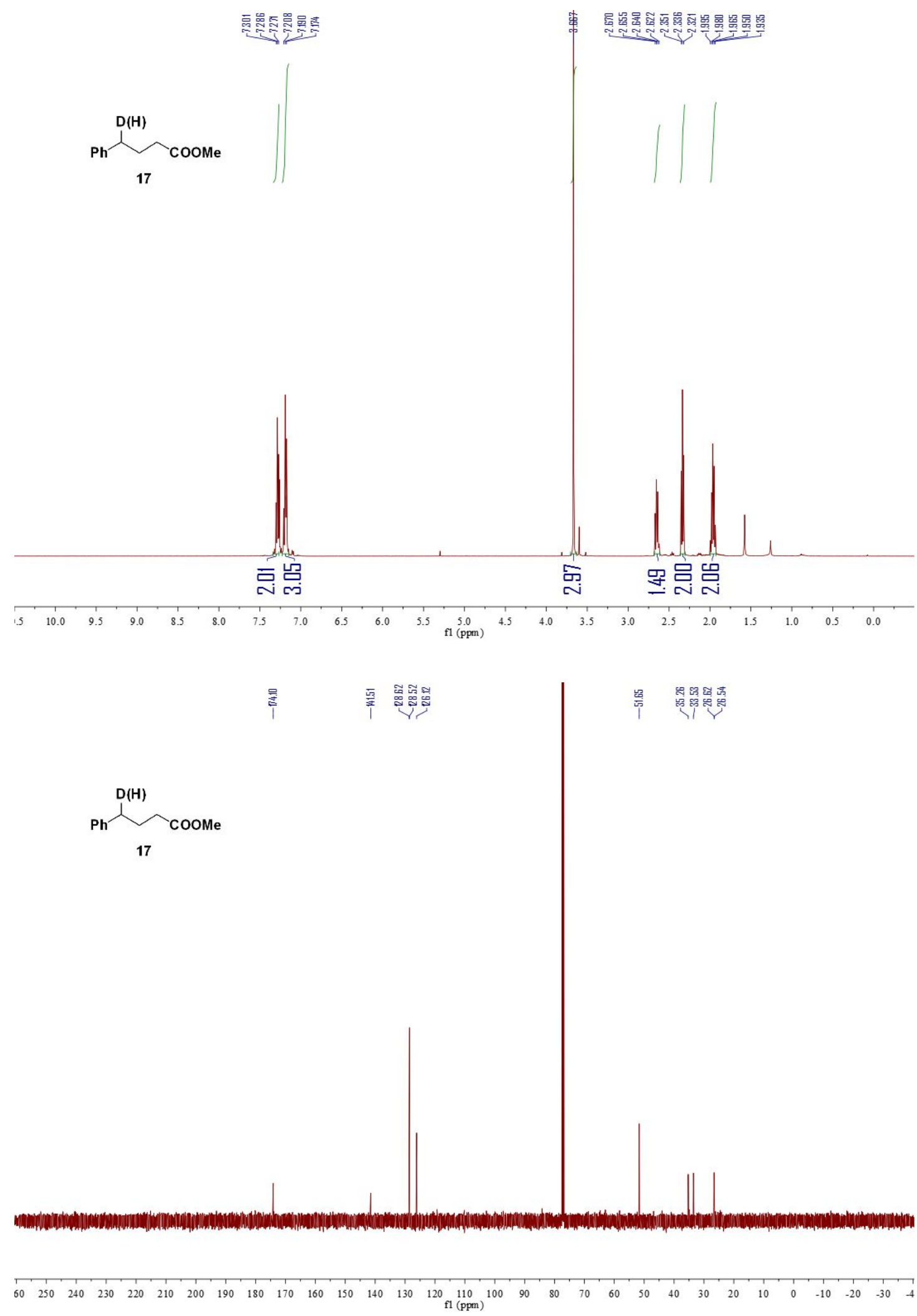




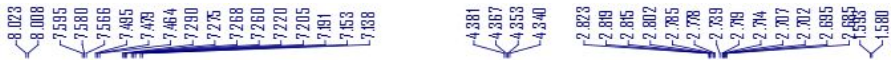
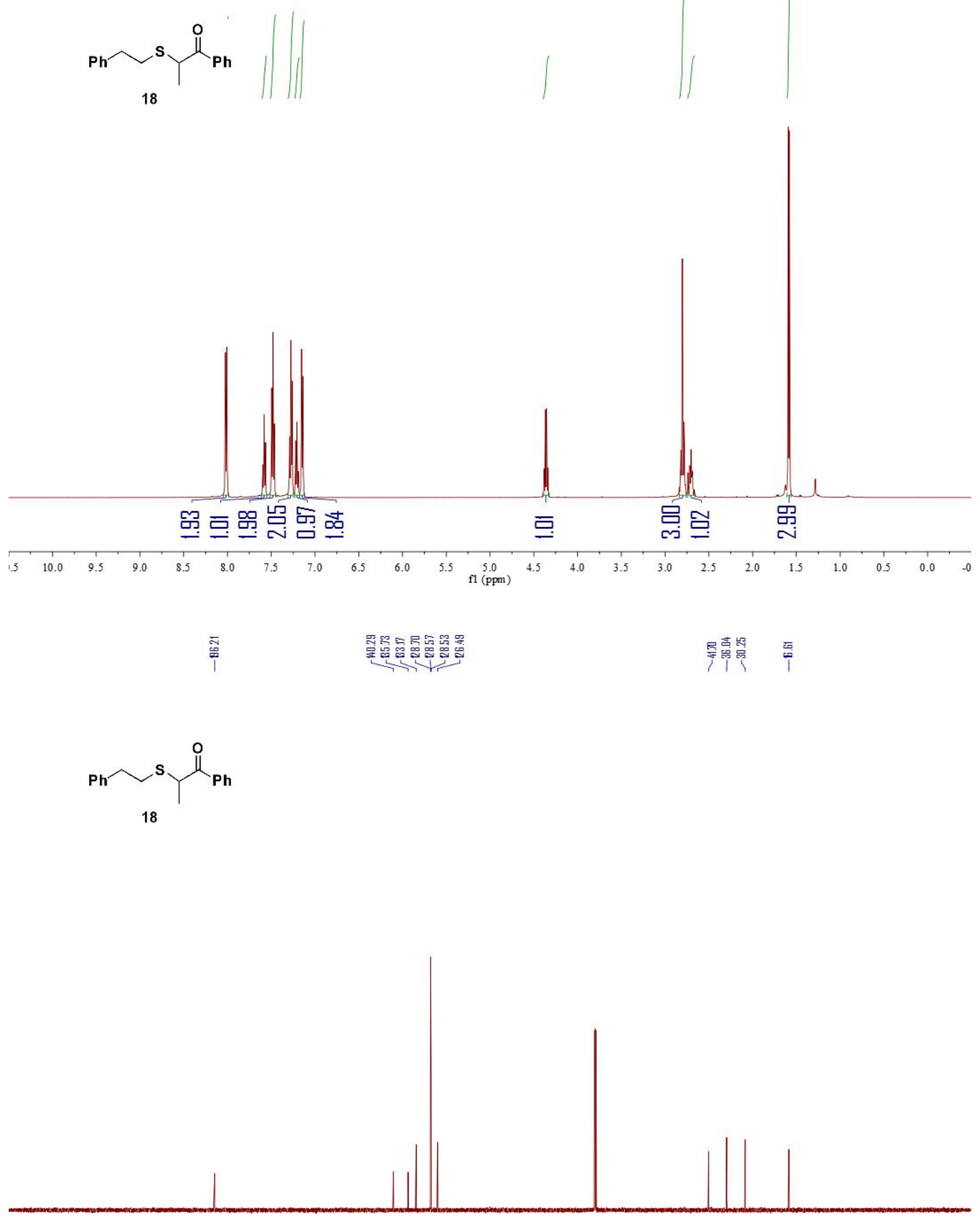

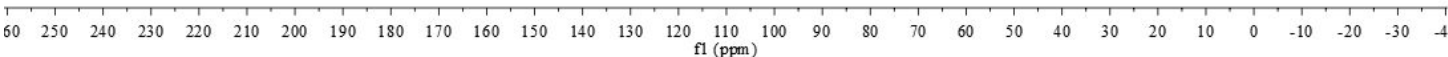




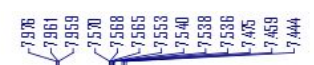

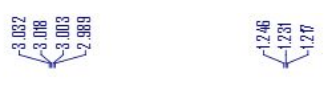

|

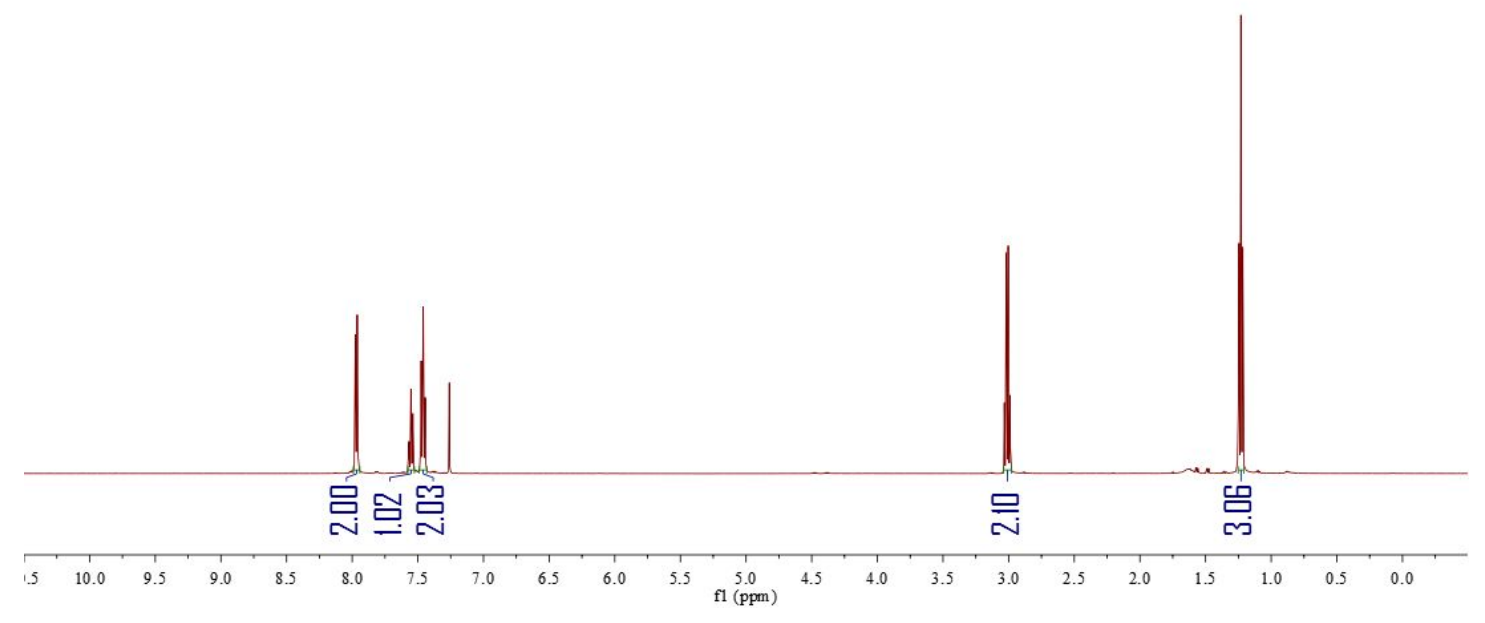

器

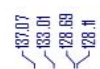

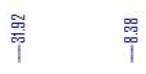

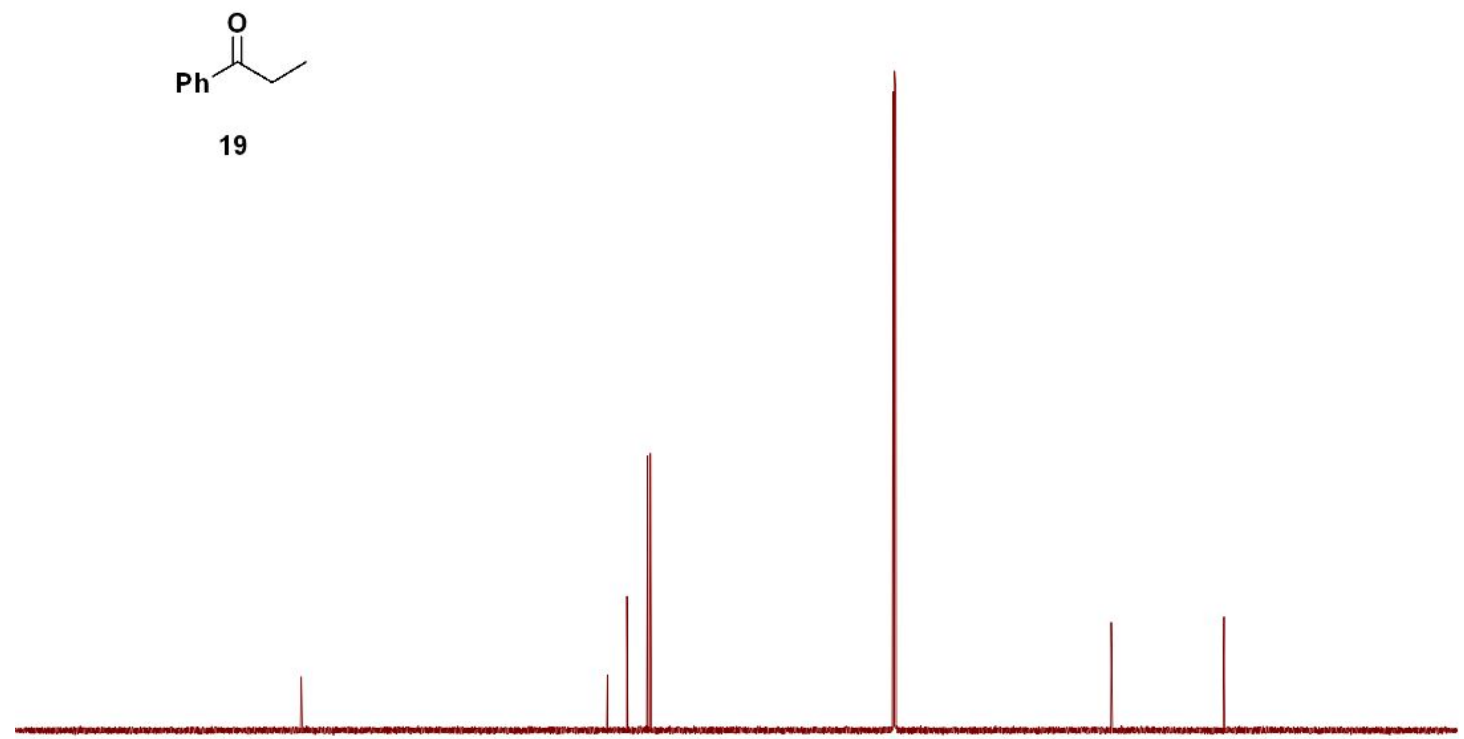

$\begin{array}{llllllllllllllllllllllllllllllllllllllllllllllllllllllll}60 & 250 & 240 & 230 & 220 & 210 & 200 & 190 & 180 & 170 & 160 & 150 & 140 & 130 & 120 & 110 & 100 & 90 & 80 & 70 & 60 & 50 & 40 & 30 & 20 & 10 & 0 & -10 & -20 & -30 & -4\end{array}$ 

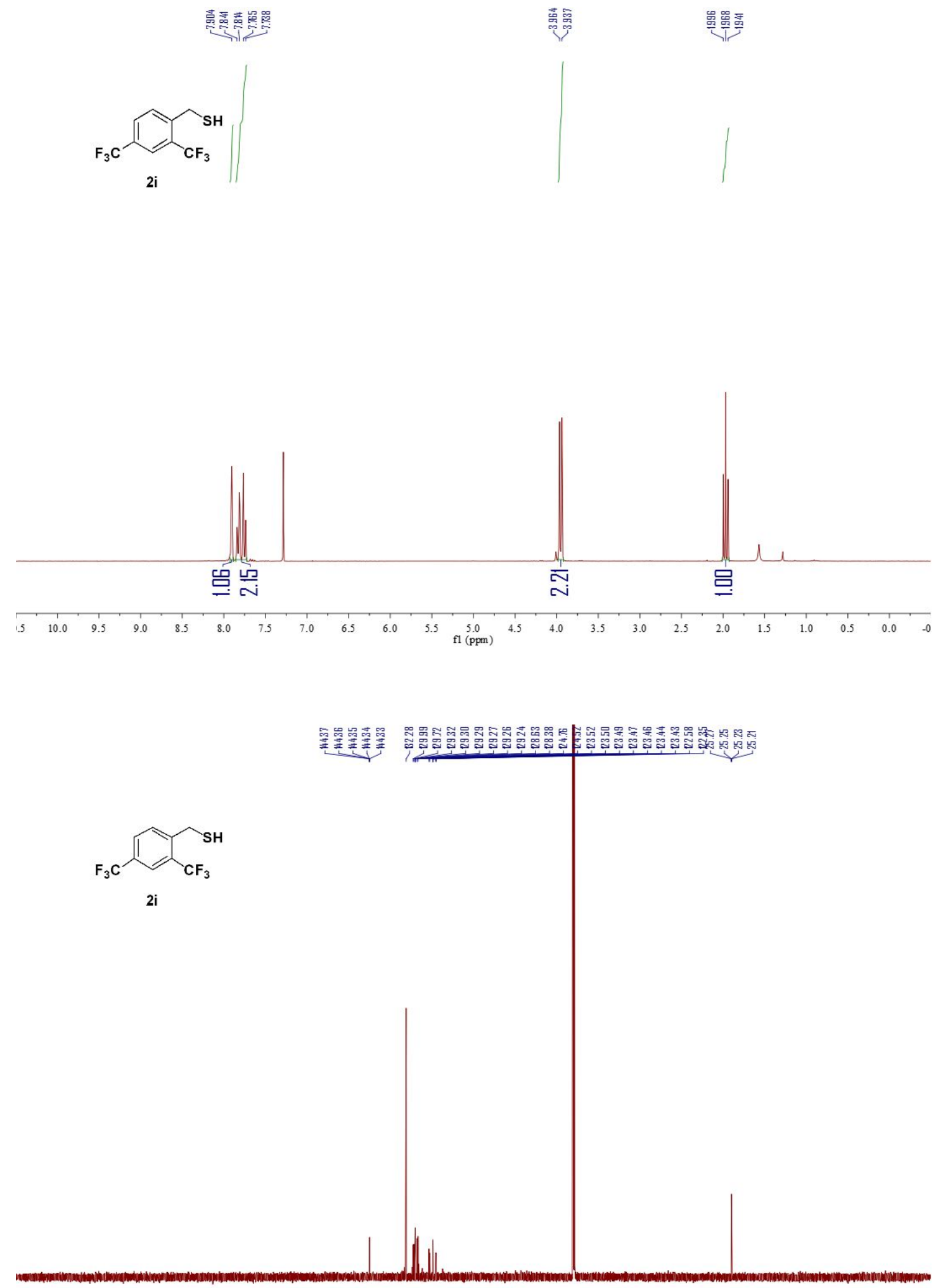

\begin{tabular}{llllllllllllllllllllllllllllllllllllllllllllllll}
\hline 60 & 250 & 240 & 230 & 220 & 210 & 200 & 190 & 180 & 170 & 160 & 150 & 140 & 130 & 120 & 110 & 100 & 90 & 80 & 70 & 60 & 50 & 40 & 30 & 20 & 10 & 0 & -10 & -20 & -30 & -4
\end{tabular} 


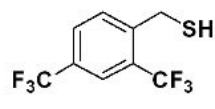

2i

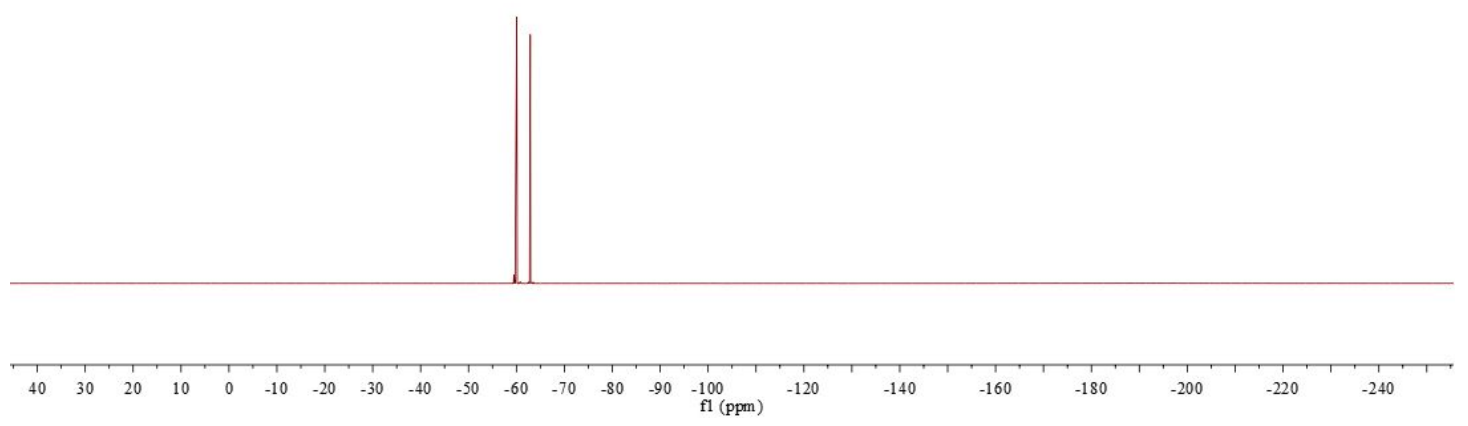

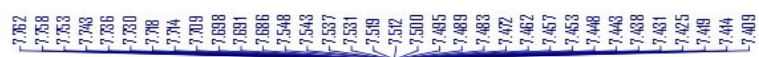
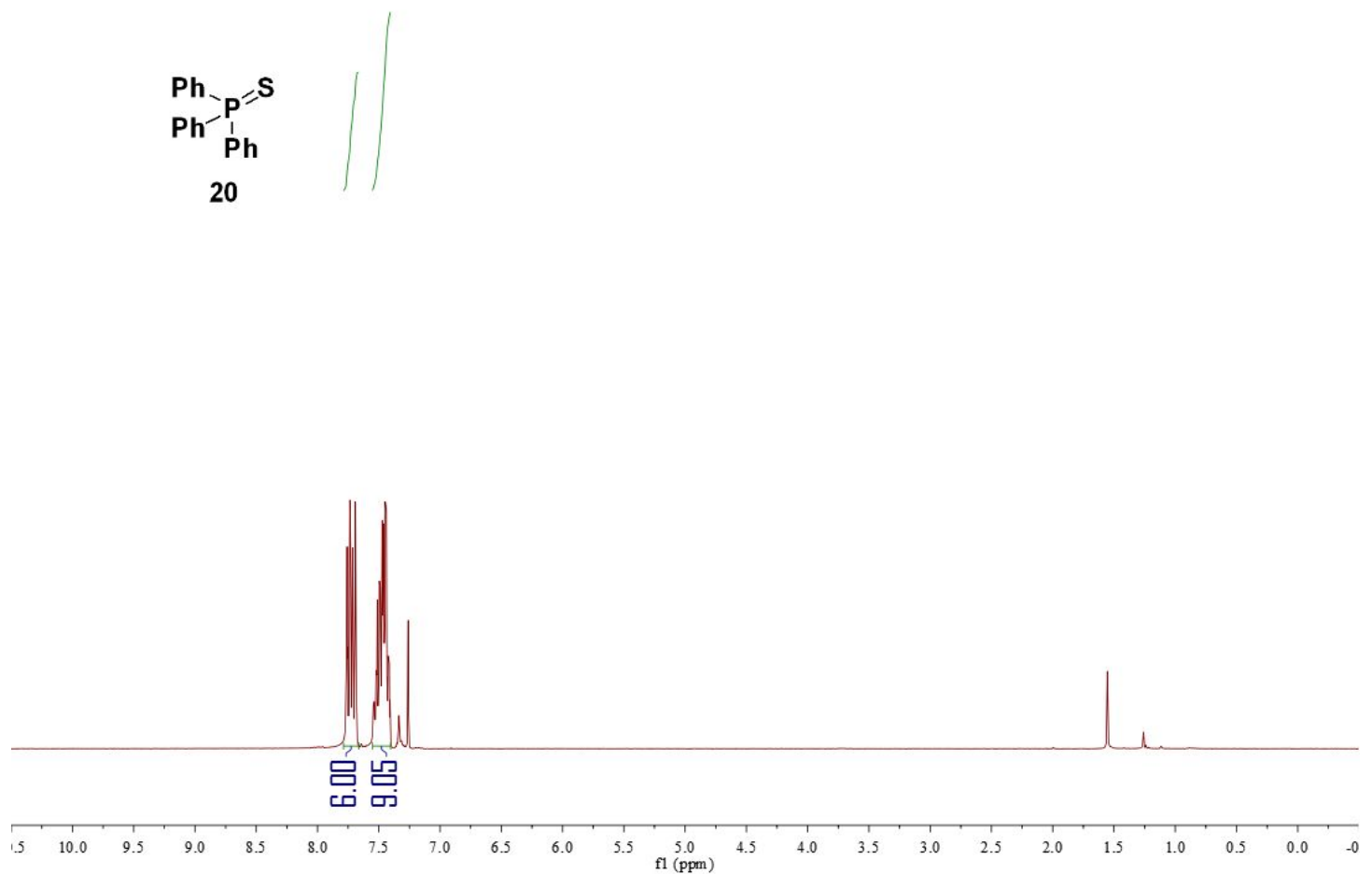


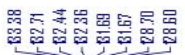

$\mathrm{Ph}_{-\mathrm{P}}=\mathrm{S}$
$\mathrm{Ph}^{-} \mathrm{Ph}$

20

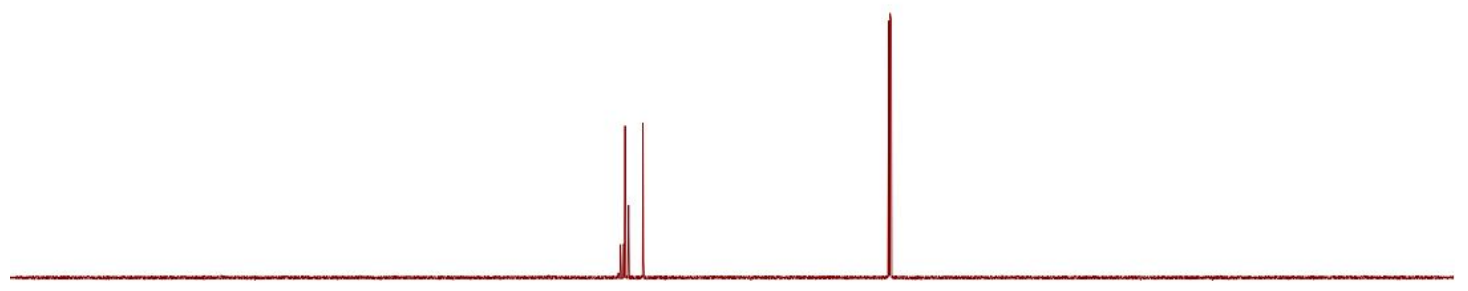

$\begin{array}{llllllllllllllllllllllllllllllllllllllll}6 & 250 & 240 & 230 & 220 & 210 & 200 & 190 & 180 & 170 & 160 & 150 & 140 & 130 & 120 & 110 & 100 & 90 & 80 & 70 & 60 & 50 & 40 & 30 & 20 & 10 & 0 & -10 & -20 & -30 & -4\end{array}$

$\mathrm{Ph}_{-\mathrm{P}}=\mathrm{S}$
$\mathrm{Ph}^{-} \mathrm{Ph}$

20

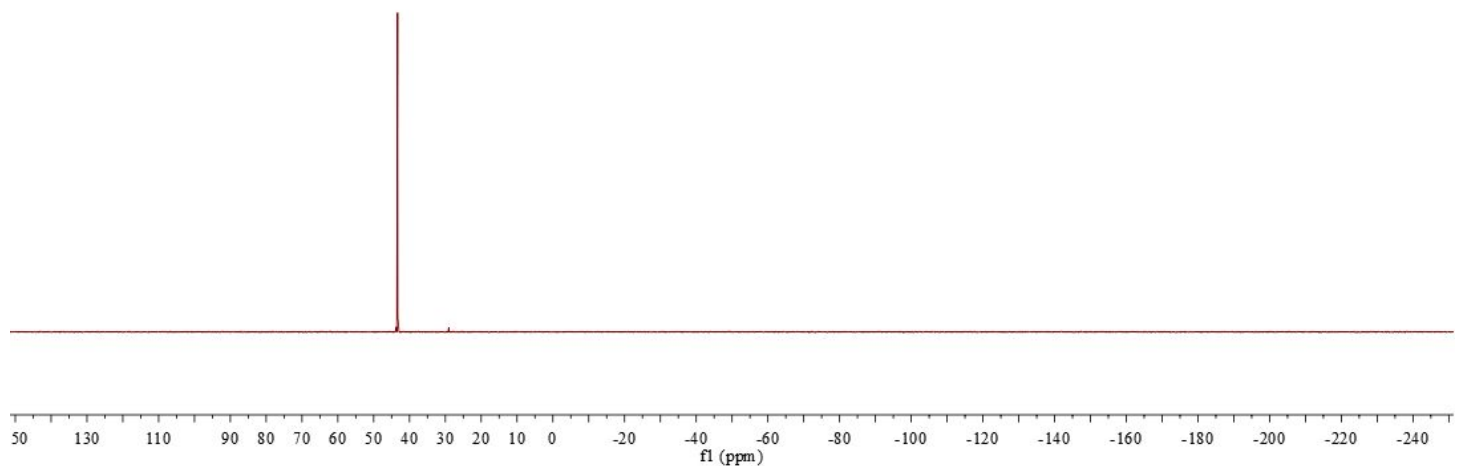




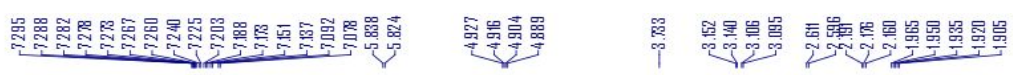

$\mathrm{IN}_{\mathrm{O}}^{\mathrm{Ph}} \mathrm{OMe}$

|| ||

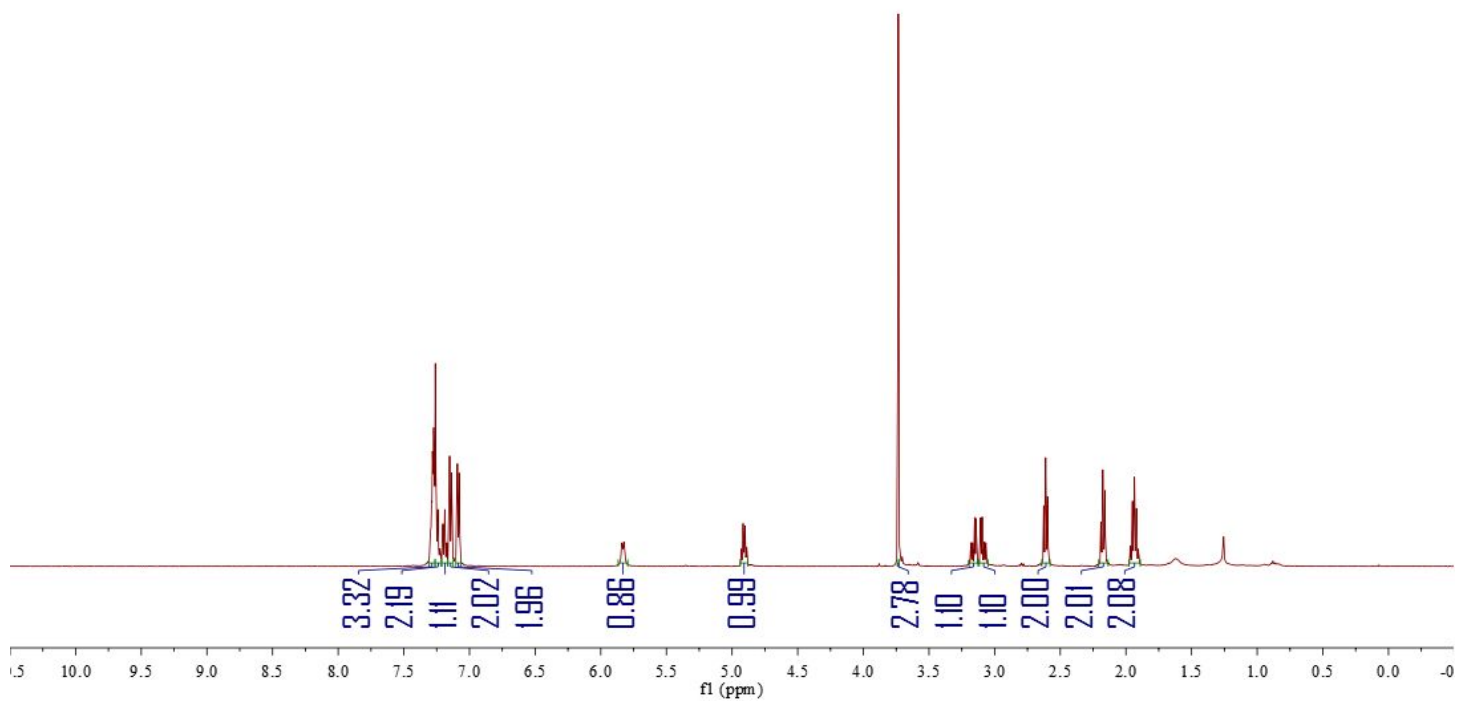

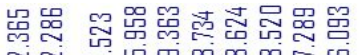

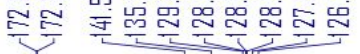

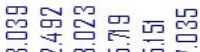

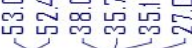

$\mathrm{Ph}_{\mathrm{H}}^{\mathrm{O}} \overbrace{\mathrm{O}}^{\mathrm{Ph}} \mathrm{OMe}$

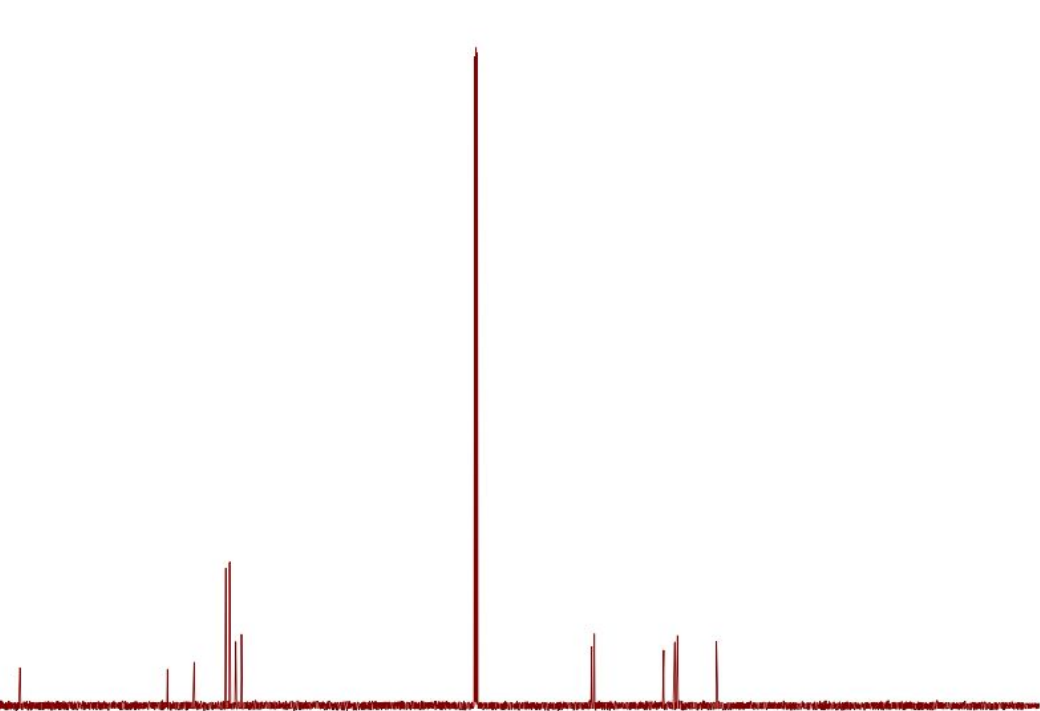

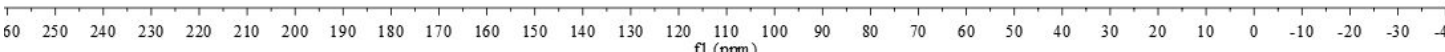




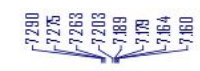

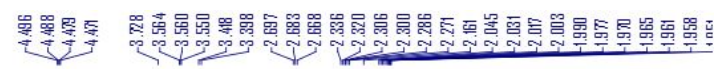

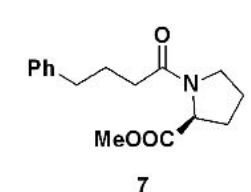

7

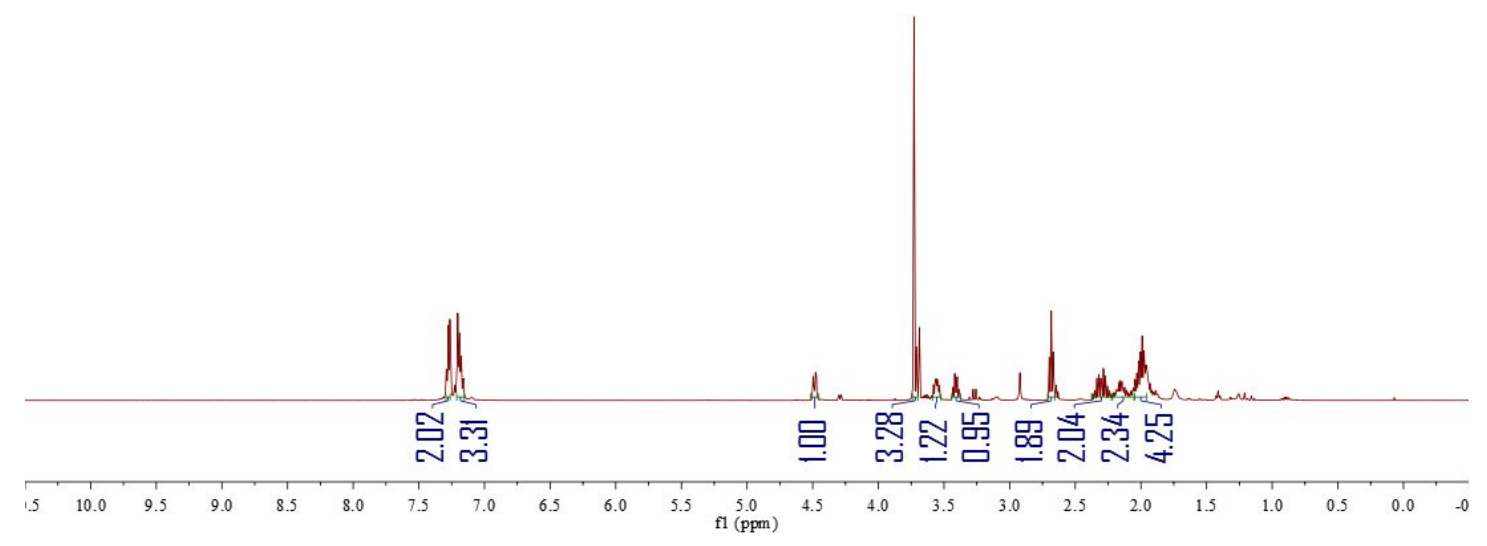

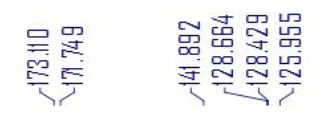

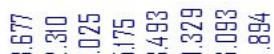

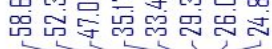
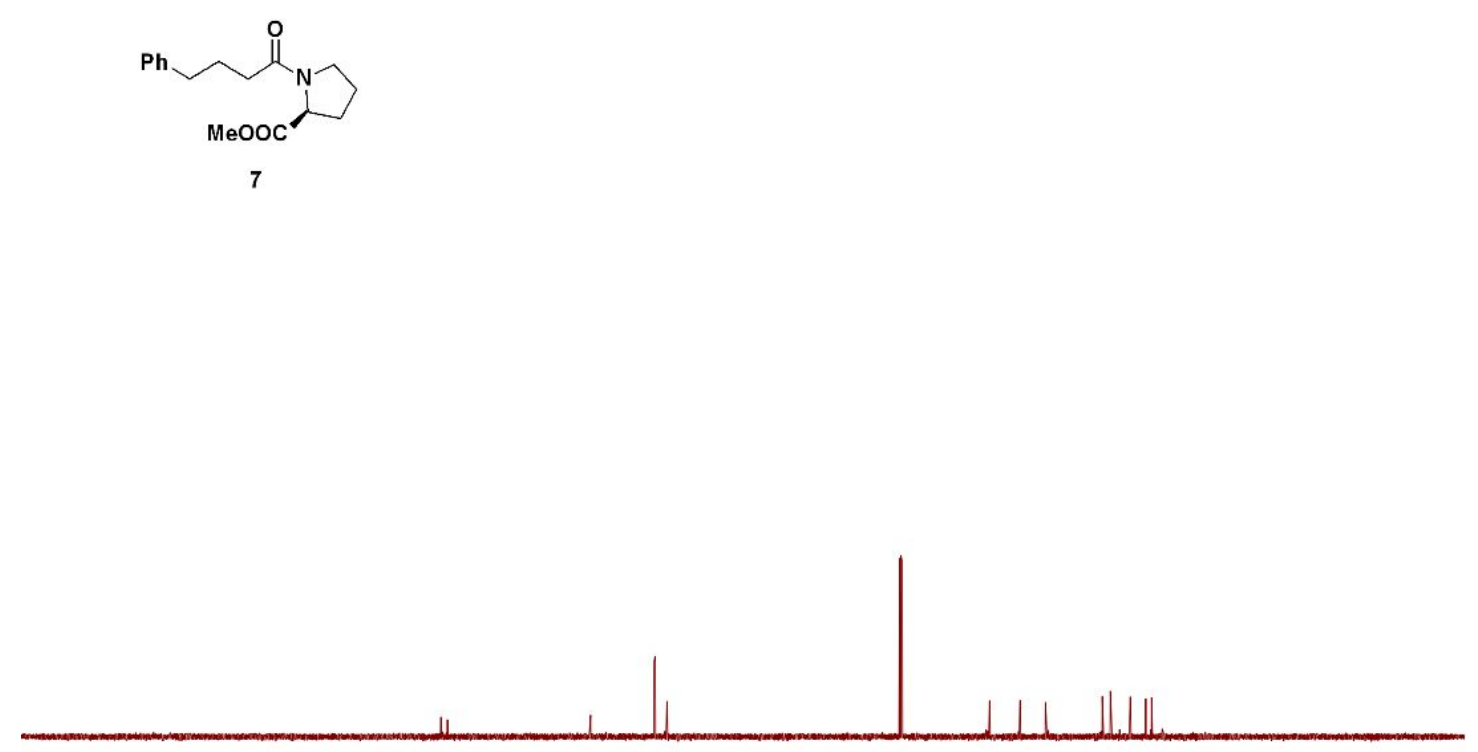

\begin{tabular}{lllllllllllllllllllllllllllllllllllllllllllllllllll}
\hline 60 & 250 & 240 & 230 & 220 & 210 & 200 & 190 & 180 & 170 & 160 & 150 & 140 & 130 & 120 & 110 & 100 & 90 & 80 & 70 & 60 & 50 & 40 & 30 & 20 & 10 & 0 & -10 & -20 & -30 & -4
\end{tabular} 

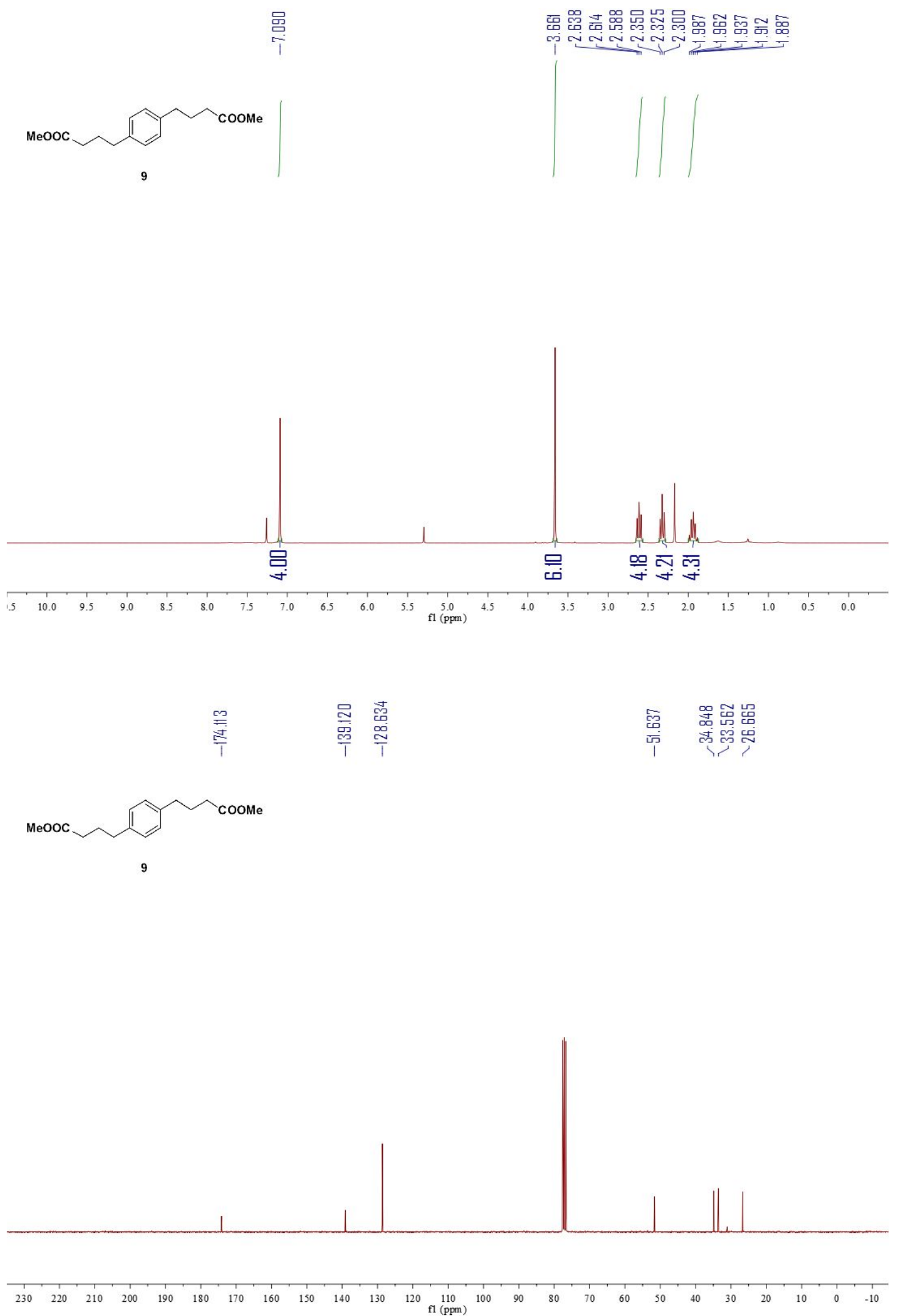


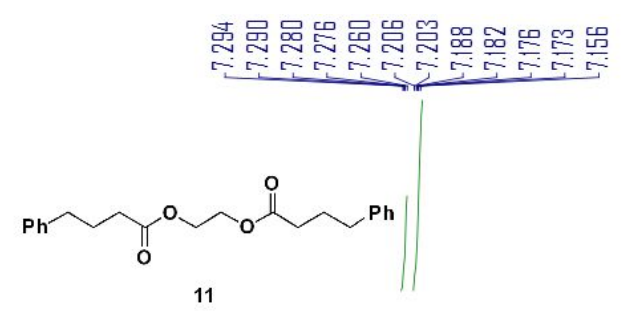

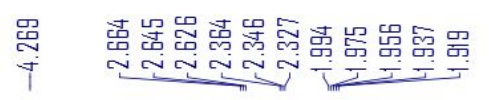
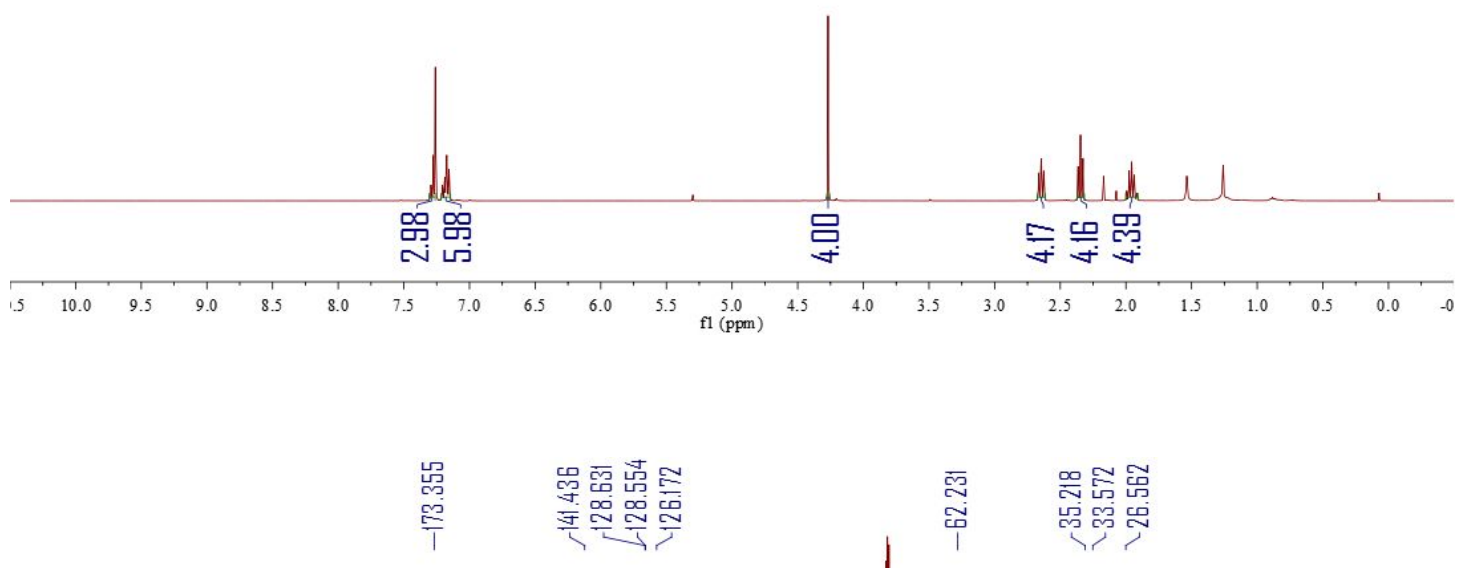

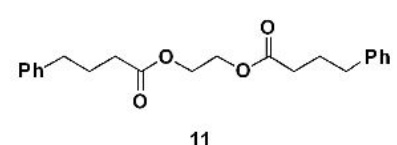

11

$\begin{array}{llllllllllllllllllllllllllllllllllllllllllllllll}260 & 250 & 240 & 230 & 220 & 210 & 200 & 190 & 180 & 170 & 160 & 150 & 140 & 130 & 120 & 110 & 100 & 90 & 80 & 70 & 60 & 50 & 40 & 30 & 20 & 10 & 0 & -10 & -20 & -30 & -40\end{array}$ 


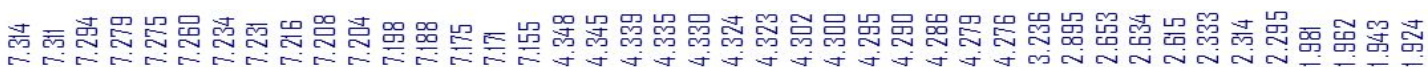
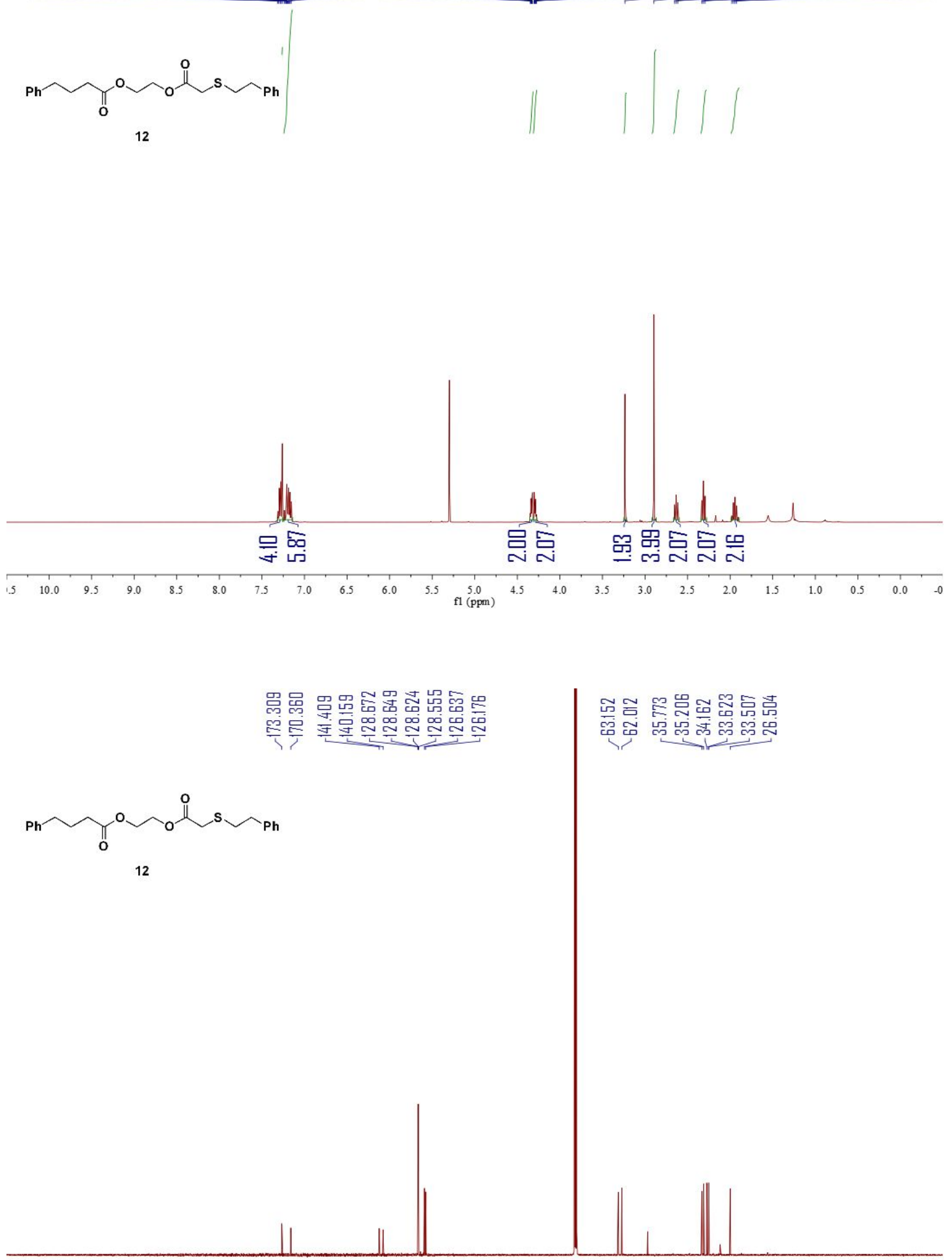

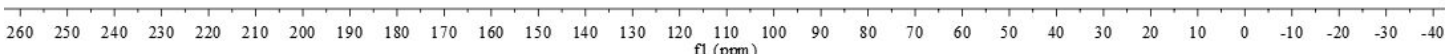



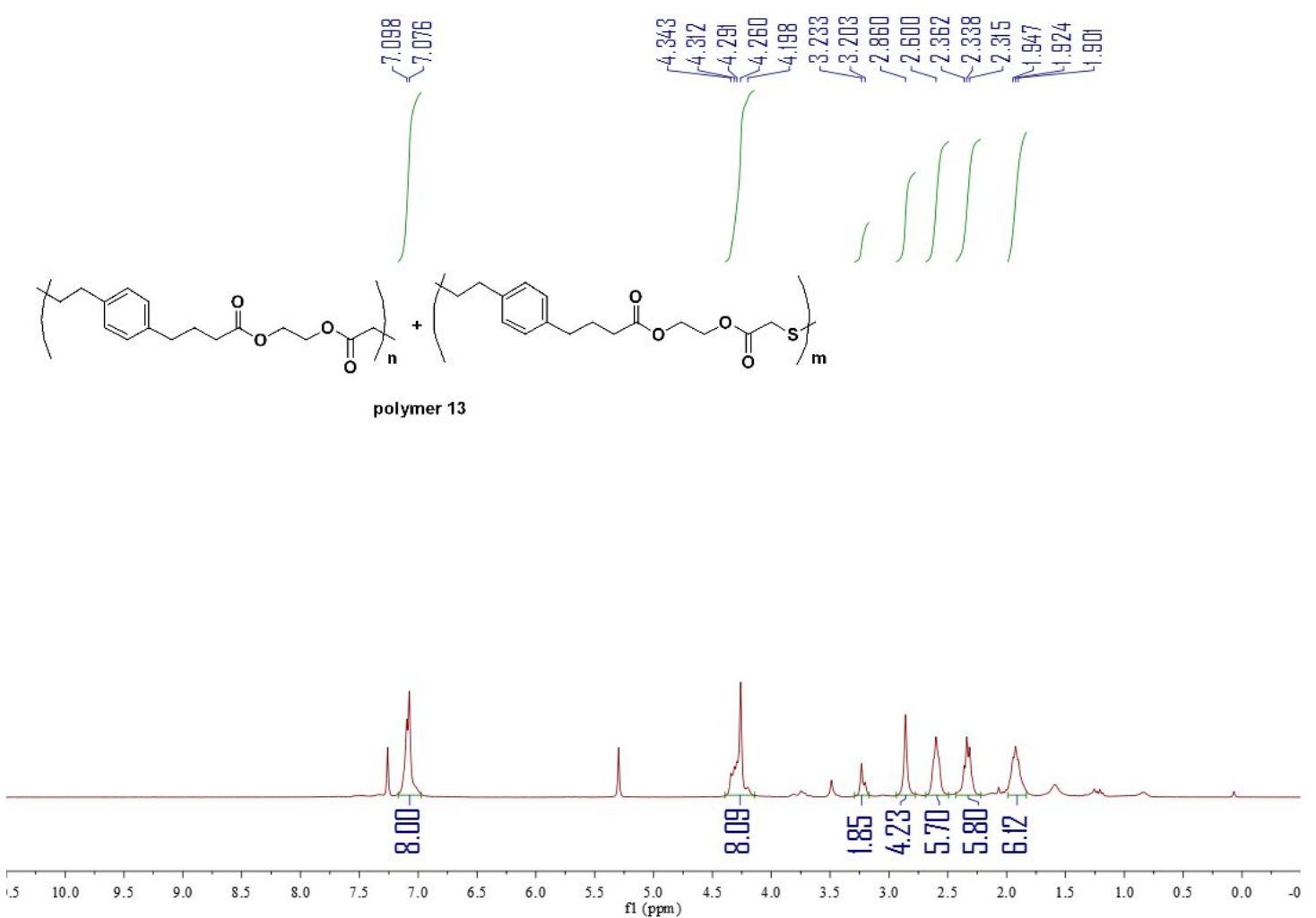

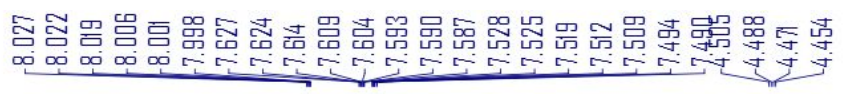
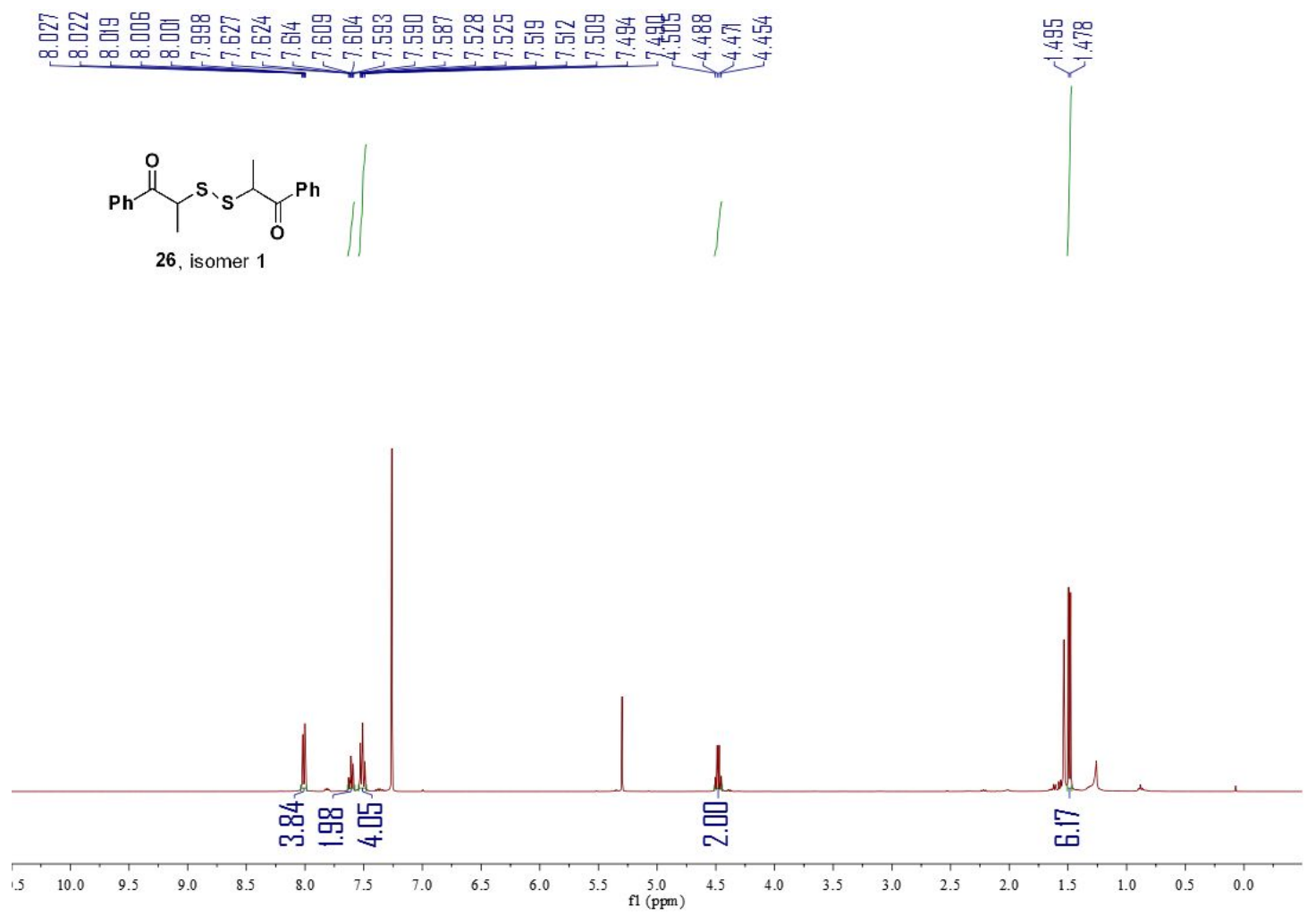

- 98 - 


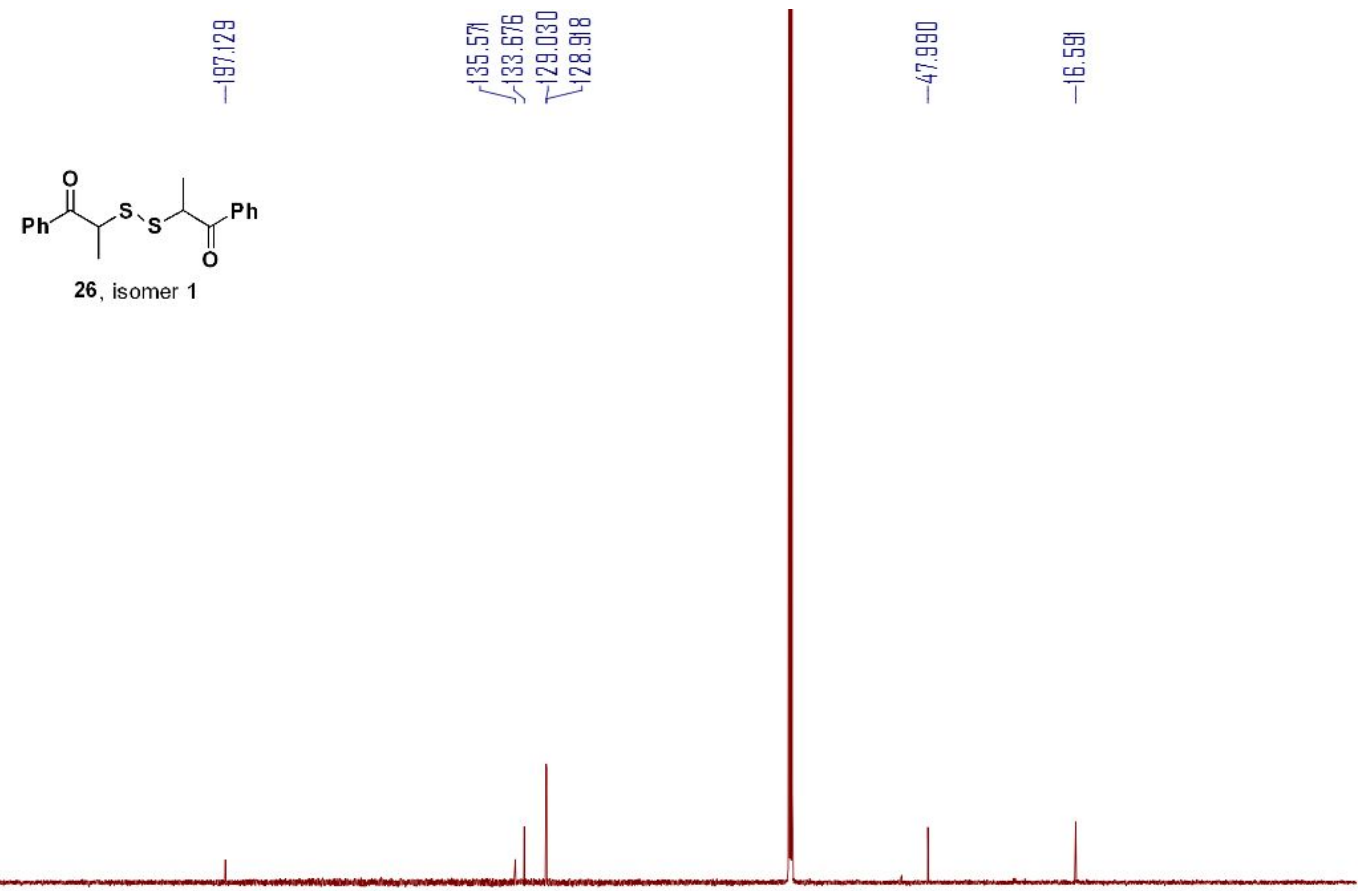

$\begin{array}{rlllllllllllllllllllllllllllllll}260 & 250 & 240 & 230 & 220 & 210 & 200 & 190 & 180 & 170 & 160 & 150 & 140 & 130 & 120 & 110 & 100 & 90 & 80 & 70 & 60 & 50 & 40 & 30 & 20 & 10 & 0 & -10 & -20 & -30 & -40\end{array}$

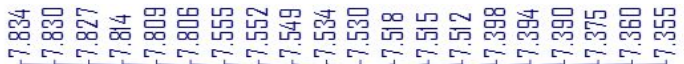
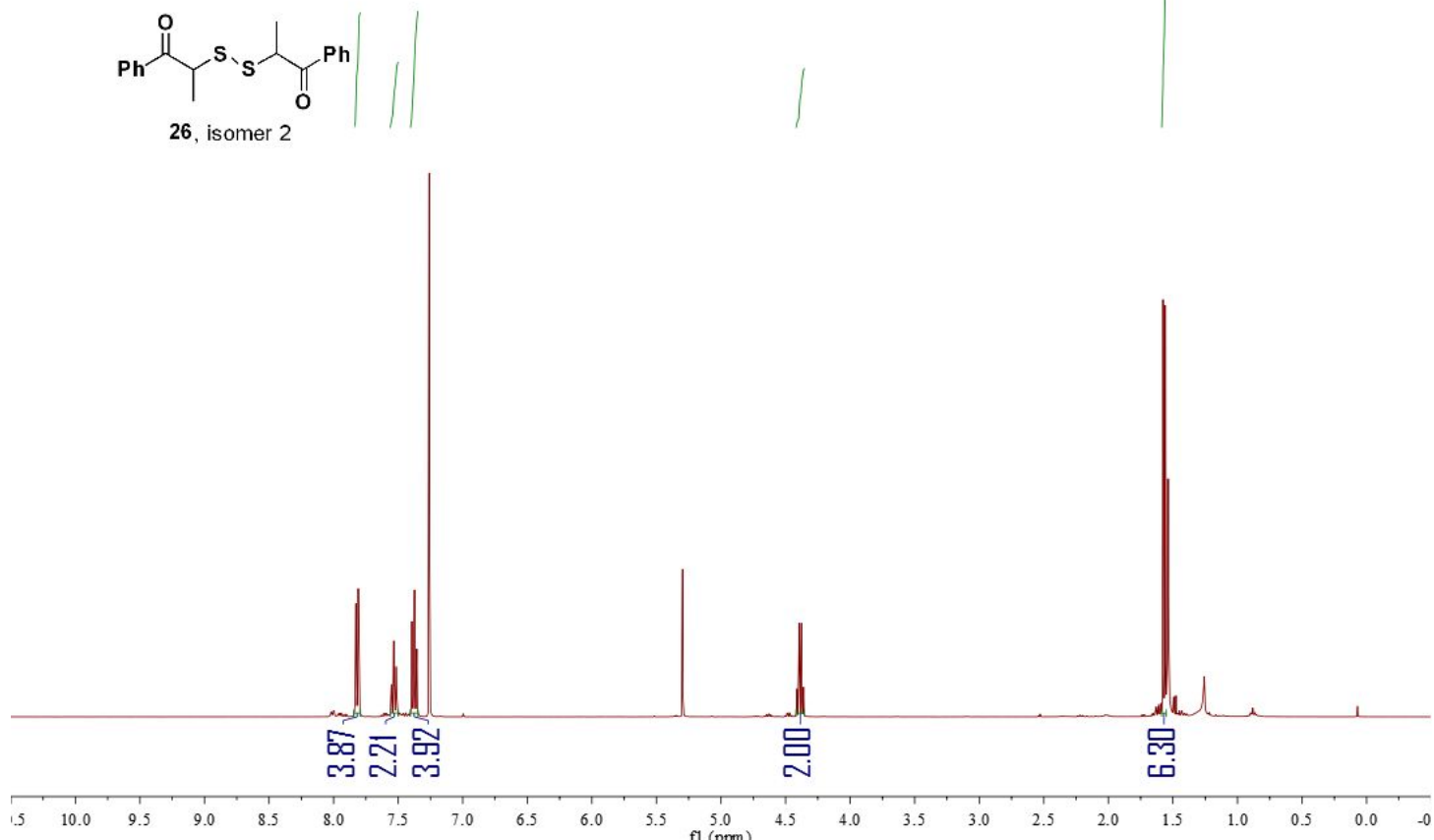


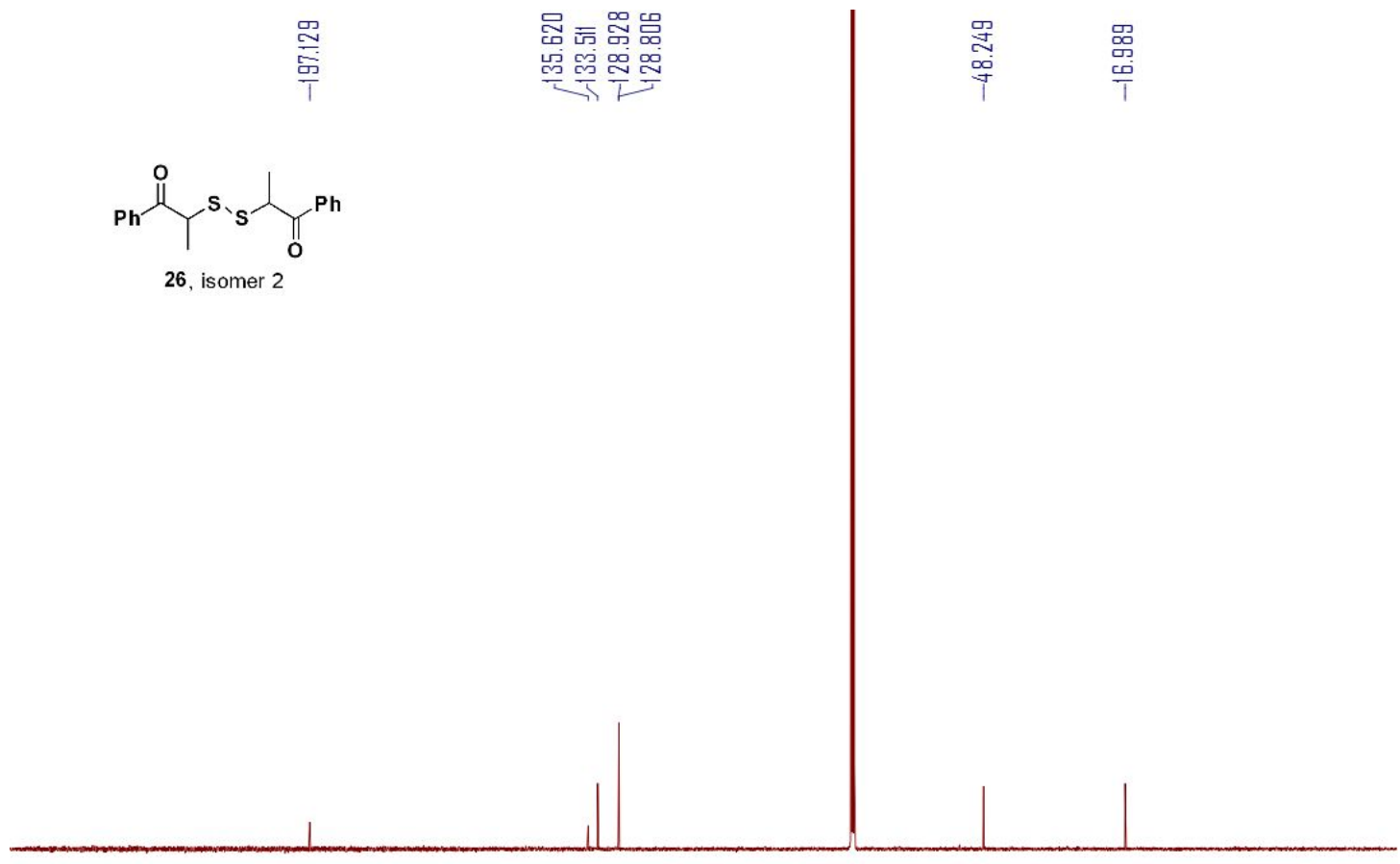

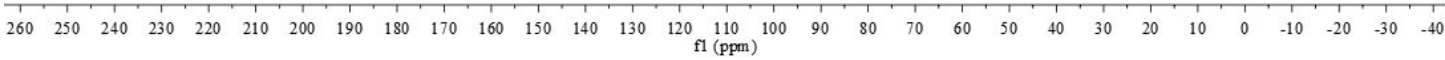
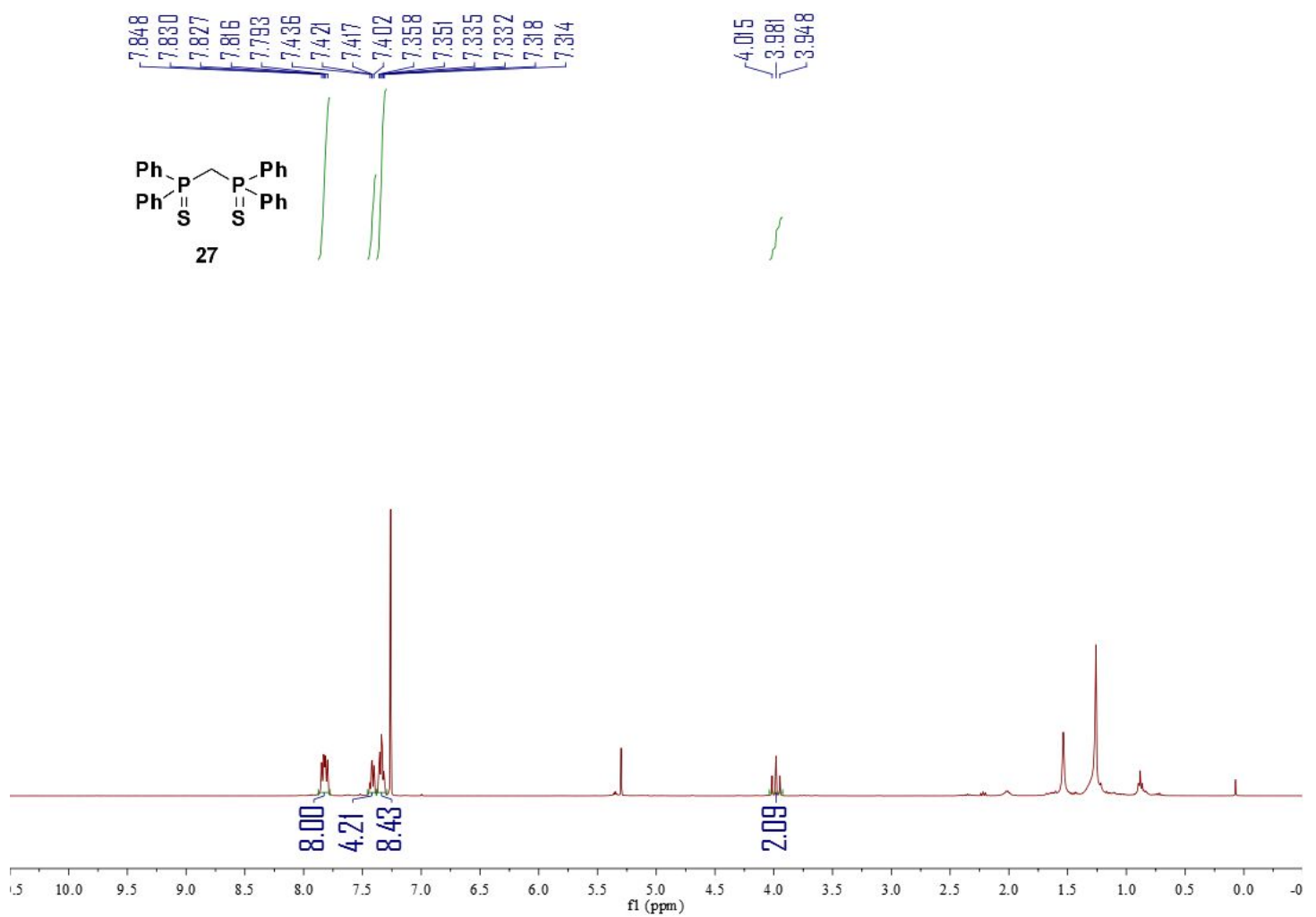
嵒

$$
\underset{27}{P^{P h} \text { II }} \overbrace{\text { II'Ph }}^{-P h}
$$

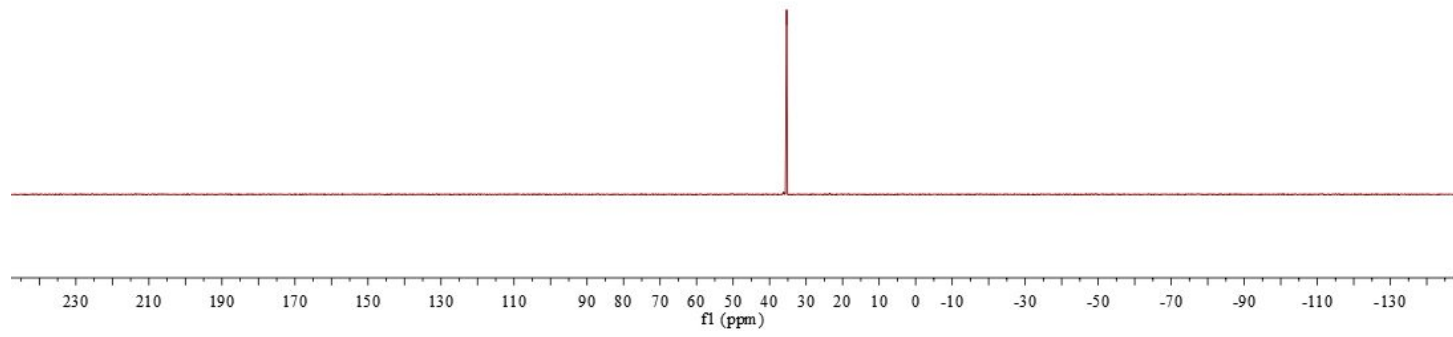

